\title{
WATER-QUALITY DATA FOR THE OHIO RIVER FROM NEW CUMBERLAND DAM TO PIKE ISLAND DAM, WEST VIRGINIA AND OHIO, JUNE-OCTOBER 1995
}

By Kimberly F. Miller and John T. Atkins

U.S. GEOLOGICAL SURVEY

Open-File Report 97-559

Prepared in cooperation with the

CITY OF NEW MARTINSVILLE, WEST VIRGINIA 


\section{U.S. DEPARTMENT OF THE INTERIOR \\ BRUCE BABBITT, Secretary \\ U.S. GEOLOGICAL SURVEY}

Gordon P. Eaton, Director

For additional information write to:

District Chief

U.S. Geological Survey, WRD

11 Dunbar Street

Charleston, WV 25301
Copies of this report can be purchased from:

U.S. Geological Survey

Branch of Information Services

Box 25286

Denver, CO 80225-0286 


\section{CONTENTS}

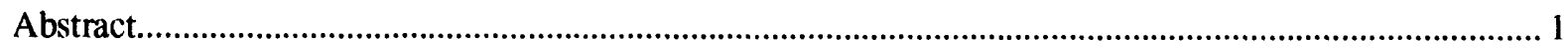

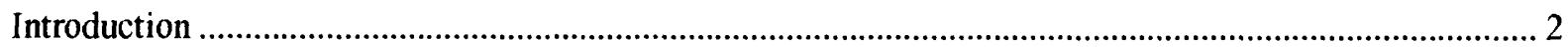

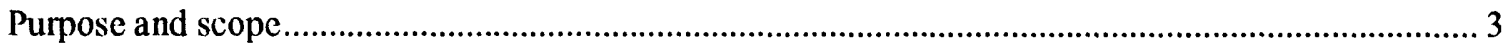

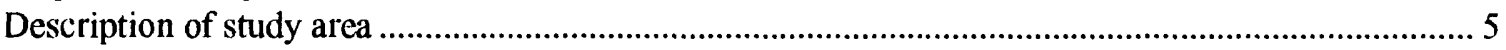

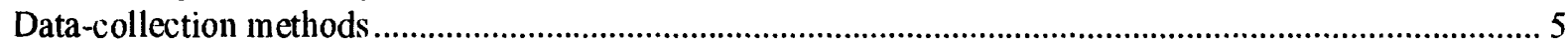

Sampling cross-sectional transects ........................................................................................ 6

Sampling longitudinal transects ................................................................................................... 6

Light-penetration measurements............................................................................................... 6

Continuous-record water-quality monitoring ………………......................................................... 9

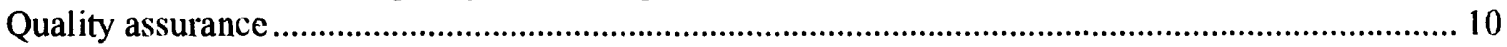

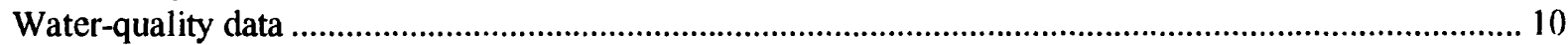

Cross-sectional and longitudinal-transect data .............................................................................. 11

Continuous-record water-quality monitoring data ............................................................................. 11

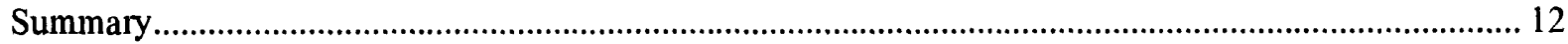

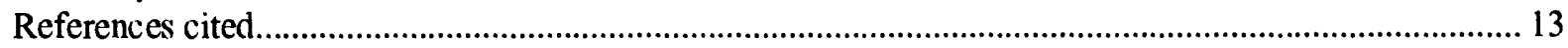

\section{FIGURES}

1-2. Maps showing:

1. Ohio River drainage basin ................................................................................................. 3

2. Ohio River study reach ....................................................................................................... 4

3a-b. Maps showing water-quality and cross-sectional sampling sites in the:

a. Upstreain section of study reach .......................................................................................

b. Downstream section of study reach......................................................................................... 8

4. Schematic diagram of New Cumberland Dam showing location of continuous-recording water-quality monitors

\section{TABLES}

1-21. Water-quality data, June through October 1994, for:

1. Station 403400080392201 , Ohio River at river mile 51.1 .............................................. 14

2. Station 403156080373201, Ohio River at river mile 54.0 …………………………...... 16

3. Station 403139080373801, Ohio River at river mile 54.4 …………………………..... 23

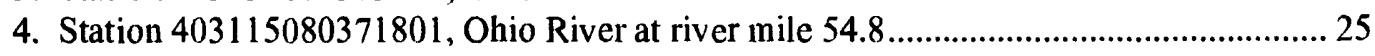

5. Station 403045080370901, Ohio River at river mile 55.4 .............................................. 31

6. Station 402930080363101 , Ohio River at river mile 57.0............................................. 32

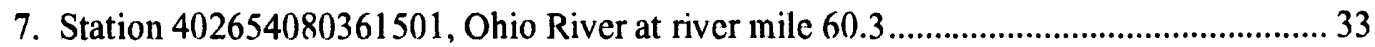

8. Station 402619080362201, Ohio River at river mile 61.0, main channel ........................ 40

9. Station 402620080364201 , Ohio River at river mile 61.0, back channel............................ 41

10. Station 4(02426080362901, Ohio River at river mile 63.2, main channel ........................ 42 


\section{TABLES--Continued}

1-22. Water-quality data, June through October 1995--Continued

11. Station 402428080364601 , Ohio River at river mile 63.2, back channel......................... 43

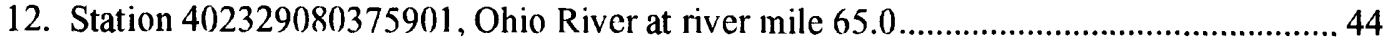

13. Station 402246080364601, Ohio River at river mile 66.4 .............................................. 45

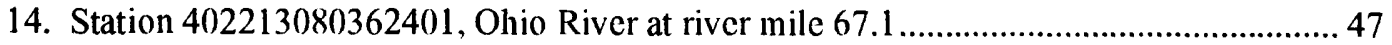

15. Station 402051080363901, Ohio River at river mile 68.7 f..............................................48

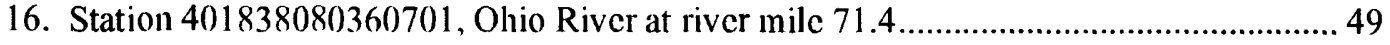

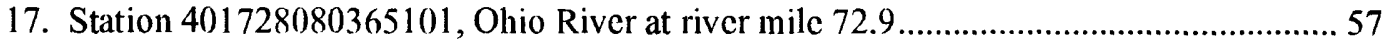

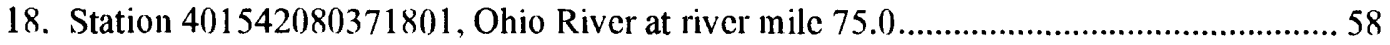

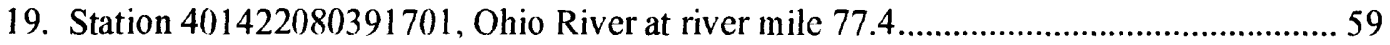

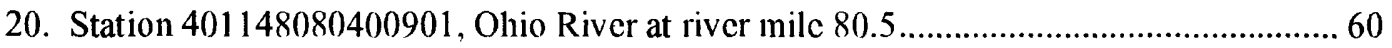

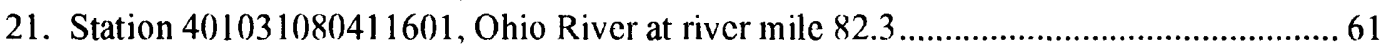

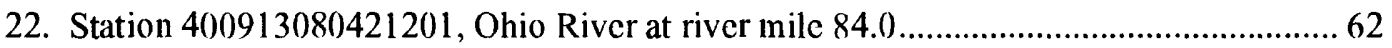

23-26. Daily maximum, minimum, and mean (median) at station 403155080373501 , from the New Cumberland Dam (upstream) continuous-recording water-quality monitor, June through October 1995, for:

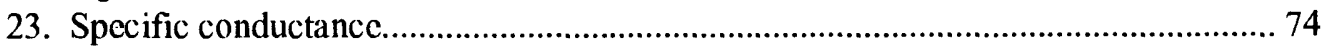

24. $\mathrm{pH}$

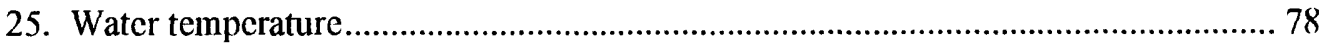

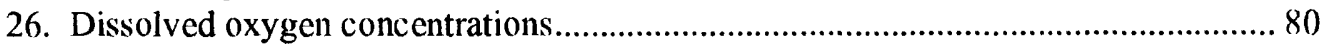

27-30. Daily maximum, minimum, and mean (median) at station 403133080372801, from the New Cumberland Dam (downstrcam) continuous-recording water-quality monitor, June through October 1995, for:

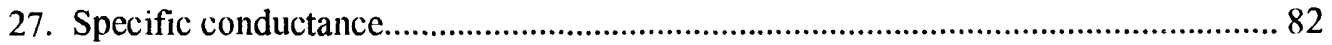

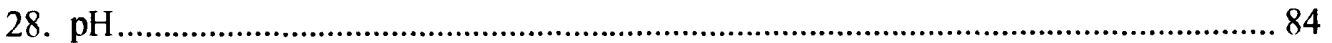

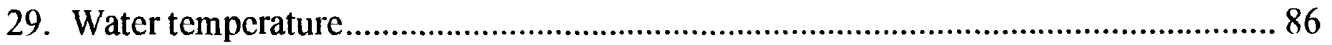

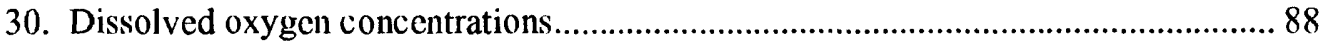




\title{
WATER-QUALITY DATA FOR THE OHIO RIVER FROM NEW CUMBERLAND DAM TO PIKE ISLAND DAM, WEST VIRGINIA AND OHIO, JUNE-OCTOBER 1995
}

\author{
By Kimberly F. Miller and John T. Atkins
}

\section{ABSTRACT}

This report contains water-quality data for the Ohio River from river mile 51.1 (3.3 miles upstream from New Cumberland Dam) to river mile 84.0 (0.2 mile upstream from Pike Island Dam) that were collected during the summer and fall of 1995 . The data were collected to define the water quality of the Ohio River and to use in assessing the proposed effects of hydropower development on the water quality of the Ohio River. Water quality was determined by a combination of synoptic field measurements and continuous-record monitoring. Water-quality characteristics were measured in the field along a longitudinal transect with 20 mid-channel sampling sites; cross-sectional transects of water-quality measurements were made at 5 of these sites. Water-quality measurements were also made at two sites located on the back-channel (Ohio) side of Browns Island and at one site near the middle of the wingwall of New Cumberland Dam. At each longitudinal-transect and back-channel sampling site, measurements of specific conductance, $\mathrm{pH}$, water temperature, and dissolved oxygen concentration were made at four depths (at the surface, about 3.0 feet below the surface, middle of the water column, and near the bottom of the river). Cross-sectional transects and the site near the middle of the wingwall of New Cumberland Dam consisted of three to four detailed vertical profiles of the same characteristics. Estimates of the depth of light penetration (Secchi disk transparency) were made at all cross-sectional sampling locations whenever light and river-surface conditions were appropriate. Synoptic sampling usually was completed in 14 hours or less and was repeated eight times between July 11 and October 17, 1995. On June 27, synoptic sampling was also done at river miles 51.1, 54.0, and 54.4; but due to equipment failure, no other samples were taken on that trip.

Continuous-record monitoring of water quality consisted of hourly measurements of specific conductance, $\mathrm{pH}$, water temperature, and dissolved oxygen concentration that were recorded at a depth of 6.6 feet at sites upstream and downstream of New Cumberland Dam. The upstream monitor was suspended from a Coast Guard buoy located approximately in the middle of the navigation channel 0.2 mile upstream from the dam. The downstream monitor was located at the end of the downstream wingwall on the riverside, about 1,200 feet from the dam. Continuousrecording monitors were operated from June through October 1995. 


\section{INTRODUCTION}

The U.S. Army Corps of Engineers has constructed and operates more than 60 lock-anddam facilities in the Ohio River Basin, with 20 facilities on the Ohio River mainstem and the rest on major tributaries in the basin (U.S. Army Corps of Engineers, 1990). The lock-and-dam structures form a system of contiguous navigation pools that ensure year-round navigation on the river. Many dams also contain hydroelectric generators that were installed after construction of the navigation structures. In 1989, the Federal Energy Regulatory Commission (FERC) issued licenses for retrofitting of hydropower at 20 dams in the upper Ohio River Basin, which includes the Allegheny and Monongahela Rivers, and the Ohio River mainstem from Pittsburgh, Pa., to Huntington, W. Va. (fig. 1). However, many of these licenses have since been surrendered.

Some dams scheduled for hydropower development currently are thought to improve the water quality of the river by increasing the rate of gas transfer from the atmosphere to the water (Federal Energy Regulatory Commission, 1988). Water from deep, slow-moving upstream pools is mixed as it passes over or through navigation structures, thereby increasing the amount of surface area in contact with the atmosphere. If the dissolved oxygen (DO) concentration is less than the saturation concentration, the potential exists for absorption of oxygen into the water, a process known as reaeration.

The amount of oxygen added to the water by reaeration at a dam depends, in part, on flow conditions of the river and design characteristics of the structure (Avery and Novak, 1978). Dams on the upper Ohio River downstream from Wheeling, W. Va., are gated structures that discharge several feet below the surface of the downstream pool and provide little reaeration (Federal Energy Regulatory Commission, 1988). Other dams, including overflow dams and gated dams with discharge above the downstream pool level, are more efficient aerators and can be important sources of DO during low-flow conditions of summer and early fall. Dams upstream from Wheeling are of the latter type. Hydropower operation at these surface-discharging structures will divert riverflow through underwater intakes where the opportunity for atmospheric gas exchange is smaller. For dams upstream from Wheeling, the loss of reaeration at low flows, combined with the oxygen consumption associated with waste assimilation and the failure of other oxygen-generating processes such as algal photosynthesis, could reduce DO concentrations below acceptable levels and diminish the wasteassimilation capacity of the river (West Virginia Department of Natural Resources, 1989).

A water-quality monitoring program was begun in 1992 in cooperation with the city of New Martinsville, W. Va., and was designed, in part, to address license requirements for development of hydropower at New Cumberland Dam (FERC Project No. 6901). This dam is located upstream from Wheeling, W. Va., and is the surfacedischarge type dam. The program uses continuous-record monitoring and synoptic sampling of water-quality characteristics near the dam and throughout the downstream navigation pool during the summer and fall to provide basic hydrologic and ecologic data on the possible environmental effects of hydropower operation. Synoptic surveys, where water-quality characteristics are analyzed quickly at many locations and depths, have been recommended for incorporation into water-quality impact assessments of proposed hydropower projects at dams and other control structures (Gulliver and others, 1990; Daniil and others, 1991). The study described in this report was conducted in the Pike Island navigation pool, a 33-mi section of the Ohio River that begins at river mile $51.1(3.3 \mathrm{mi}$ upstream from New Cumberland Dam) and extends downstream to river mile $84.0(0.2 \mathrm{mi}$ upstream from Pike Island Dam) (fig. 2). 


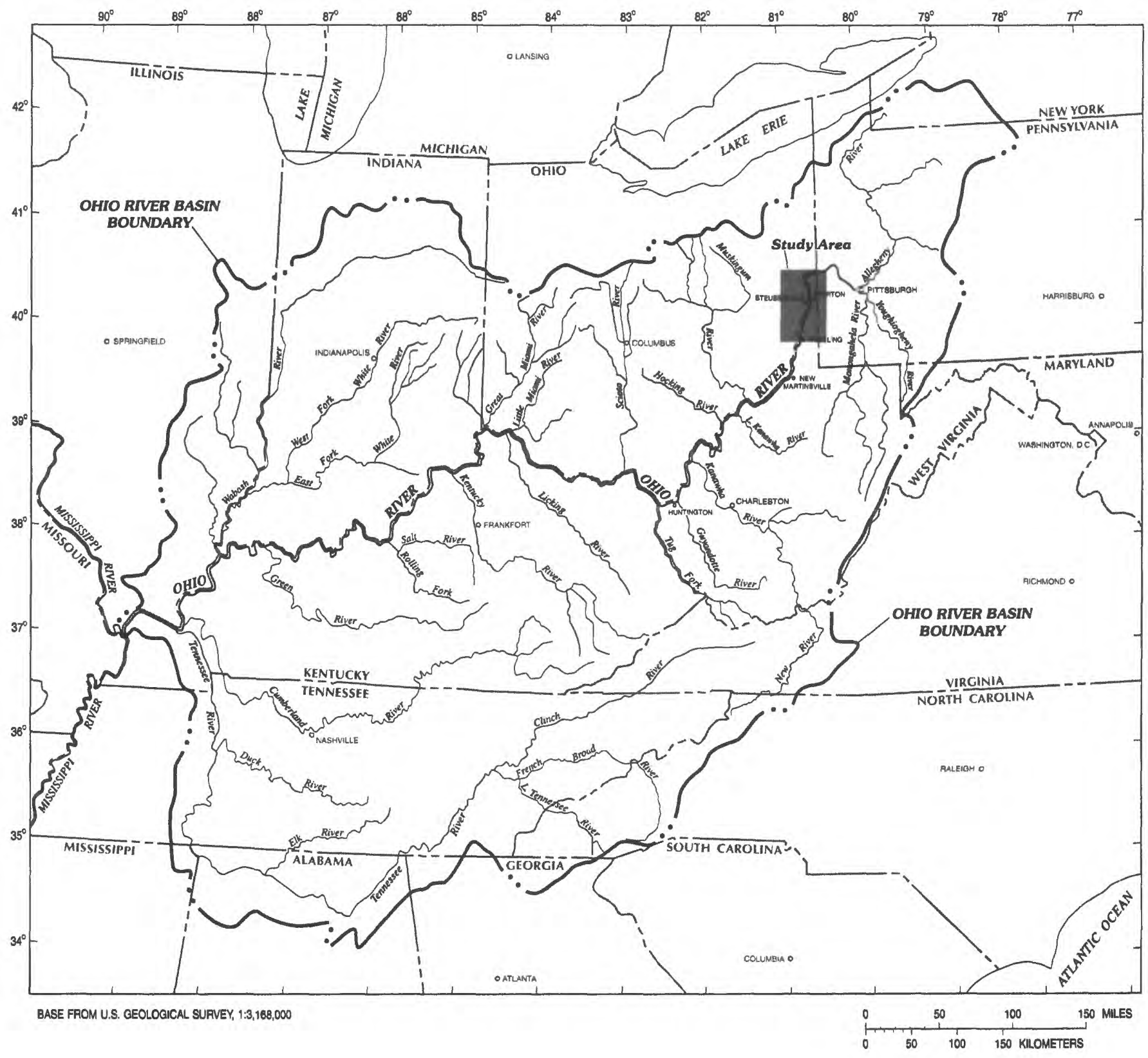

Flgure 1. Ohio River drainage basin.

\section{Purpose and Scope}

This report presents data collected in 1995 on the spatial and temporal distribution of selected water-quality characteristics in the Pike Island Pool of the Ohio River (the reach of river from New Cumberland Dam at the upstream end to Pike Island Dam at the downstream end). This report contains water-quality data of the Pike Island Pool determined by continuous-record monitoring of conditions near New Cumberland Dam and by repeated synoptic sampling of the entire 33-mi pool. Measurements of specific conductance, $\mathrm{pH}$, water temperature, and DO concentration were recorded by the continuous-recording monitors and were made at each sampling site in the network during synoptic-sampling periods. Water samples also were collected from four depths at various sampling sites. In addition to these measurements, 


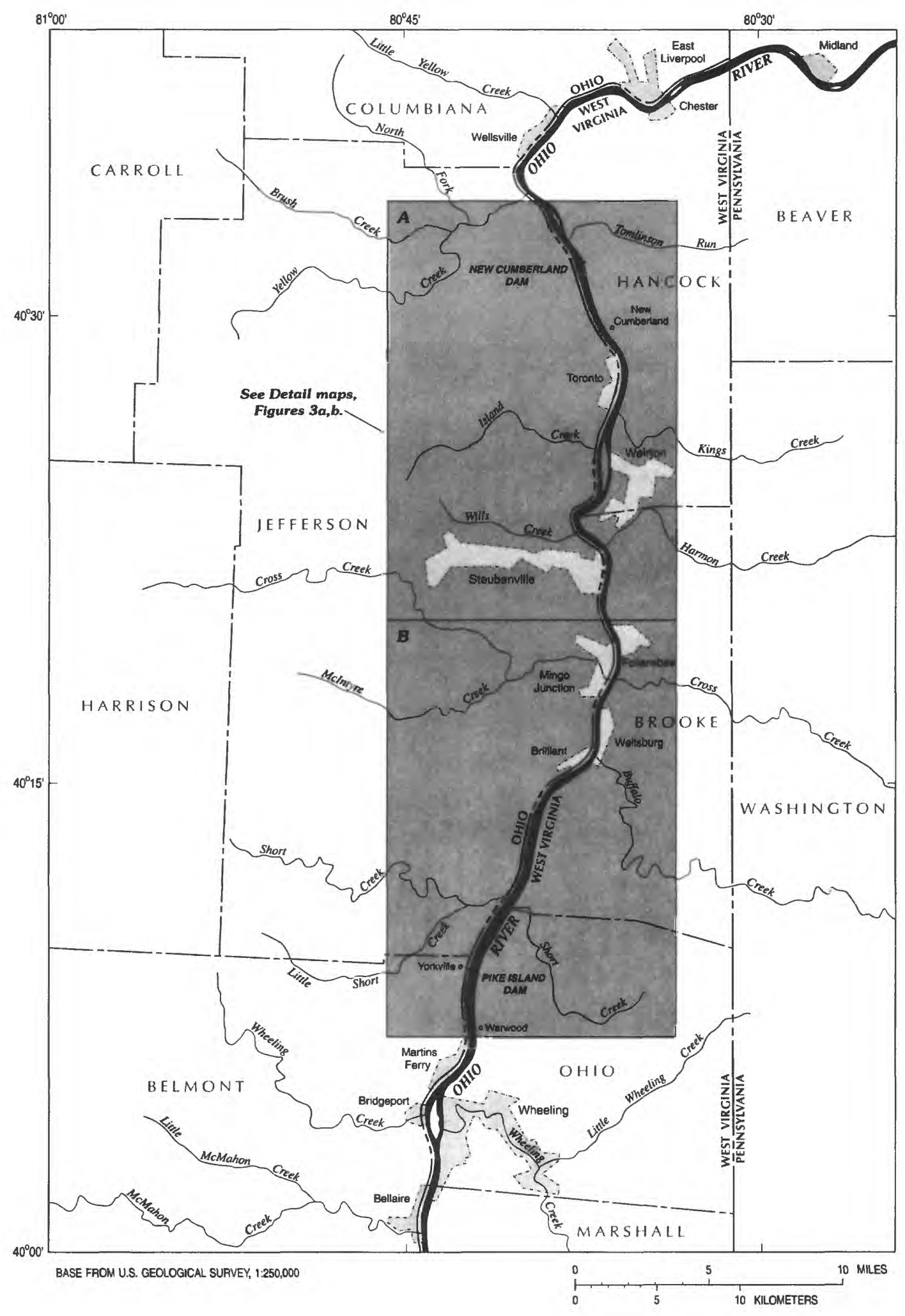

Figure 2. Ohio River study reach. 
estimates of the depth of light penetration (Secchi disk transparency) were made at cross-sectional sampling locations whenever light and riversurface conditions were appropriate. Synoptic water-quality measurements were made twice in July, August, September, and October 1995. A partial data set was collected on June 27 , consisting of river miles 51.1,54.0, and 54.4.

\section{Description of Study Area}

Drainage area for the Ohio River at Pike Island Dam is $24,700 \mathrm{mi}^{2}$. Most of the drainage basin up to the dam consists of narrow flood plains and deeply incised tributary valleys. The basin is underlain by bedrock that consists of shale, sandstone, siltstone, limestone, and coal (West Virginia Department of Natural Resources, 1988). The average width of the Pike Island pool is $1,338 \mathrm{ft}$. The average bottom slope is $0.4 \mathrm{ft} / \mathrm{mi}$; the average depth of the pool is $19 \mathrm{ft}$ (Ohio River Valley Water Sanitation Commission, 1988).

Streamflow in the upper Ohio River Basin is related to precipitation and to the balance of precipitation and evapotranspiration. The climate of the region is temperate with distinct seasonal changes. Mean minimum air temperatures $\left(-7.3^{\circ} \mathrm{C}\right)$ are generally during January; mean maximum air temperatures $\left(28^{\circ} \mathrm{C}\right)$ are generally during July. Average annual air temperature is about $12^{\circ} \mathrm{C}$. Annual precipitation in the basin ranges from 20 to 72 in., with heaviest amounts occurring in June or July and minimum amounts occurring in October (West Virginia Department of Natural Resources, 1988). The U.S. Army Corps of Engineers has constructed a system of multipurpose reservoirs on four main tributaries for flood control. These reservoirs also are used to augment flow and maintain navigation during critical periods.

Land use in the study area is about 15 percent cropland, 9 percent pasture, 46 percent forest, 6 percent urban, and 24 percent other uses (Ohio River Valley Water Sanitation Commission, 1988). Major urban and industrial centers in the reach include Toronto, Ohio, Wierton, W. Va., Steubenville, Ohio, Follansbee, W. Va., Mingo Junction, Ohio, and Wellsburg, W. Va. (fig. 2). The reach includes three municipal drinking-water intakes (Toronto, river mile 59.1; Weirton, river mile 65.1; and Steubenville, river mile 65.2) and 23 industrial intakes. The States of West Virginia and Ohio have issued permits for 13 municipal and 34 industrial effluent discharges in the study reach. Industrial activity along the reach is associated mainly with steel manufacturing, coal preparation, and coal-fired electric-power generation. This section of the river is also used to transport coal, petroleum products, chemicals, and other materials. Seven river terminals handling petroleum products and hazardous chemicals are located in the study reach (Ohio River Valley Water Sanitation Commission, 1988).

\section{DATA-COLLECTION METHODS}

Water quality of the Pike Island pool was determined by a combination of synoptic field measurements and continuous-record monitoring. A partial data set of synoptic field measurements for river miles 51.1, 54.0, and 54.4 were collected on June 27. Complete datasets of synoptic field measurements were made on July 11, July 25, August 8, August 22, September 7-8, September 22 , October 3, and October 17, 1995. Two continuous-recording monitors were in operation at New Cumberland Dam from June 1 through October 30, 1995.

The field-data-collection network used for synoptic sampling consisted of a longitudinal transect with 20 mid-channel sampling sites; crosssectional transects of water-quality characteristics were made at 5 of these sites. Water quality also was measured at two sites located on the backchannel (Ohio) side of Browns Island and at one site near the middle of the wingwall of New Cumberland Dam. At each longitudinal-transect and back-channel sampling site, measurements of specific conductance, $\mathrm{pH}$, water temperature, and dissolved oxygen concentration were made at four depths (at the surface, about $3.0 \mathrm{ft}$ below the surface, middle of the water column, and near the bottom of the river). Cross-sectional transects consisted of three to four detailed vertical profiles of the same characteristics. Also, a detailed vertical profile of the above parameters was obtained at the sampling site near the middle of the wingwall. Estimates of the depth of light 
penetration (Secchi disk transparency) were made at cross-sectional sampling locations whenever light and river-surface conditions were appropriate. Synoptic sampling of the entire network usually was completed in 14 hours or less.

\section{Sampling Cross-Sectional Transects}

During each sampling period, water quality was measured in cross-sectional transects at five locations shown in figures $3 \mathrm{a}$ and $3 \mathrm{~b}$. Two cross sections were located near New Cumberland Dam, at the ends of the upstream and downstream wingwalls (river miles 54.0 and 54.8 , respectively) (fig. 3a). Additional cross-sections were located $4.9 \mathrm{mi}$ upstream from the industrial complex at Steubenville, Ohio, (river mile 60.3) (fig. 3a) and 6.2 mi downstream from Steubenville (river mile 71.4) (fig. 3b). One cross-sectional transect was located at Pike Island Dam at the end of the upstream wingwall (river mile 84.0) (fig. 3b). As weather permitted, the cross sections at river miles $60.3,71.4$, and 84.0 consisted of near-sunrise cross-sectional transect measurements of water quality. These same sampling sites were also measured during the afternoon of the same day of the near-sunrise measurements.

The downstream cross-sectional transect at New Cumberland Dam and the single crosssectional transect at Pike Island Dam consisted of four vertical profiles of specific conductance, $\mathrm{pH}$, water temperature, and DO concentration measurements. Positions for the vertical profiles at the downstream cross-sectional transect at New Cumberland Dam were located by estimating 25 , 50,75 , and 100 percent of the distance from the left bank to edge of the wingwall. Positions for the vertical profiles at the single cross-sectional transect at Pike Island Dam were located at approximately $1 \mathrm{ft}$ from the edge of the wingwall and by estimating 25,50 , and 75 percent of the distance from the edge of the wingwall to the right bank. Both were sampled in random order to minimize effects of diel changes (changes associated with a 24-hour period which includes both day and night). Cross-sectional transects at other locations consisted of three vertical profiles, with positions determined by estimating 25,50 , and 75 percent of the total width of the river. Weather and river-surface conditions occasionally prevented completion of all vertical profiles in a transect. Vertical-profile measurements were made at the surface, at $3.0 \mathrm{ft}$, and $5.0 \mathrm{ft}$, and then at depth intervals of $5.0 \mathrm{ft}$, using a portable, multiparameter water-quality monitoring system (Hydrolab ${ }^{1}$ Surveyor 3). Measuring was begun either at the bottom of the river or at the surface. Barometric pressure was recorded before making each set of field-data measurements by use of a Thommen TX altimeter-barometer.

\section{Sampling Longitudinal Transects}

Longitudinal transects consisted of making measurements of specific conductance, $\mathrm{pH}$, water temperature, and DO concentration at four depths (at the surface, about $3.0 \mathrm{ft}$ below the surface, middle of the water column, and near the bottom of the river) at 20 mid-channel sampling sites distributed throughout the Pike Island pool. Two additional sampling sites were located on the backchannel (Ohio) side of Browns Island. A sampling site also was near the middle of the wingwall of New Cumberland Dam where measurements were made at the surface, at $3.0 \mathrm{ft}$ and $5.0 \mathrm{ft}$, and then in $5.0-\mathrm{ft}$ intervals until near the bottom of the river. The locations of the sampling sites are shown in figures $3 \mathrm{a}$ and $3 \mathrm{~b}$. Each location corresponds to the position of a U.S. Coast Guard navigation light or daymark. Sampling methods and instruments were the same as for the cross-sectional transects.

\section{Light-Penetration Measurements}

At each cross-sectional sampling site, an estimate of the depth of light penetration was made lowering a 9-in.-diameter Secchi disk into the

1. The use of brand, firm, or trade names in this report is for identification purposes and does not constitute endorsement by the U. S. Geological Survey. 


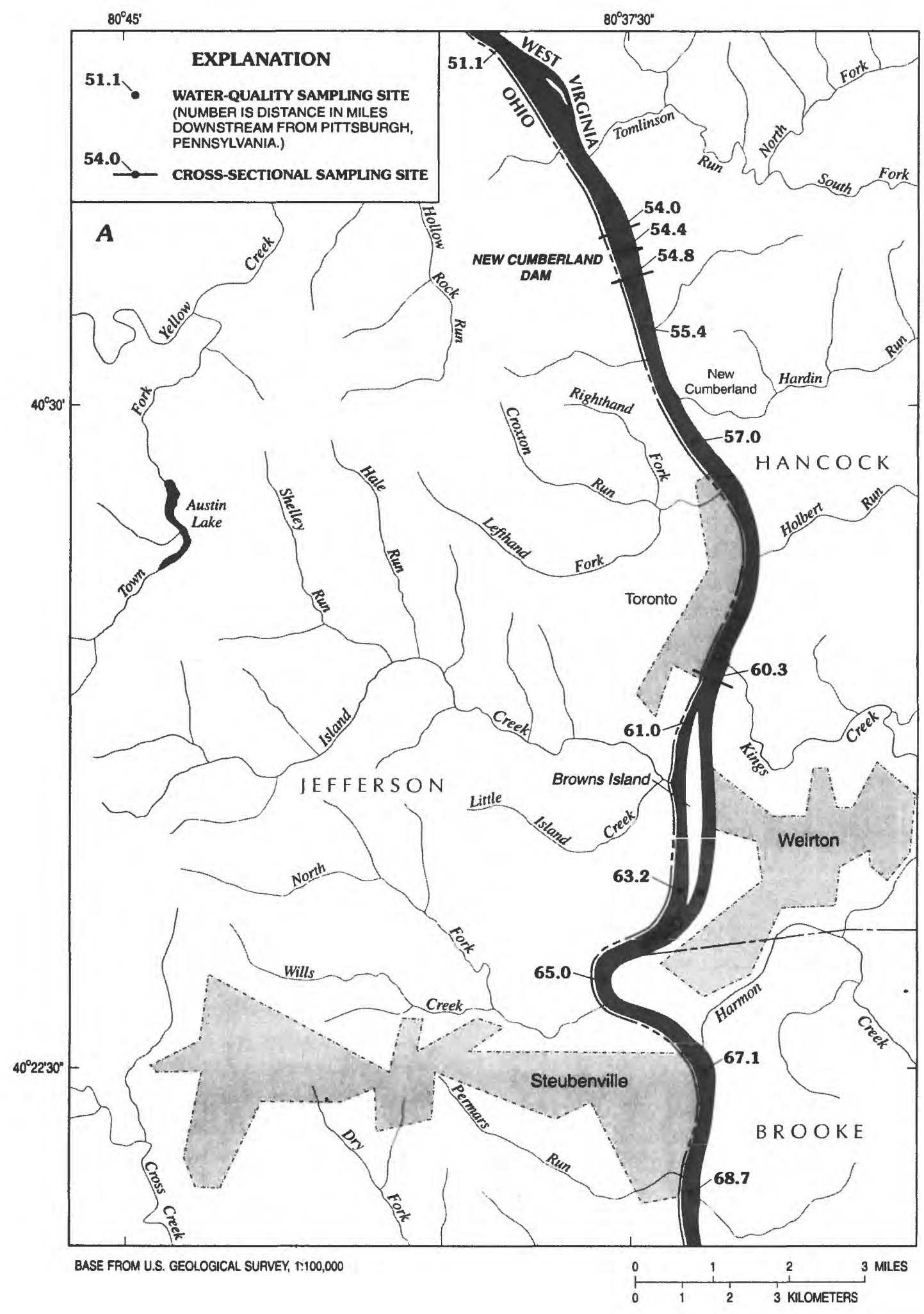

Figure 3a. Water-quality and cross-sectional sampling sites in the upstream section of the study reach. 


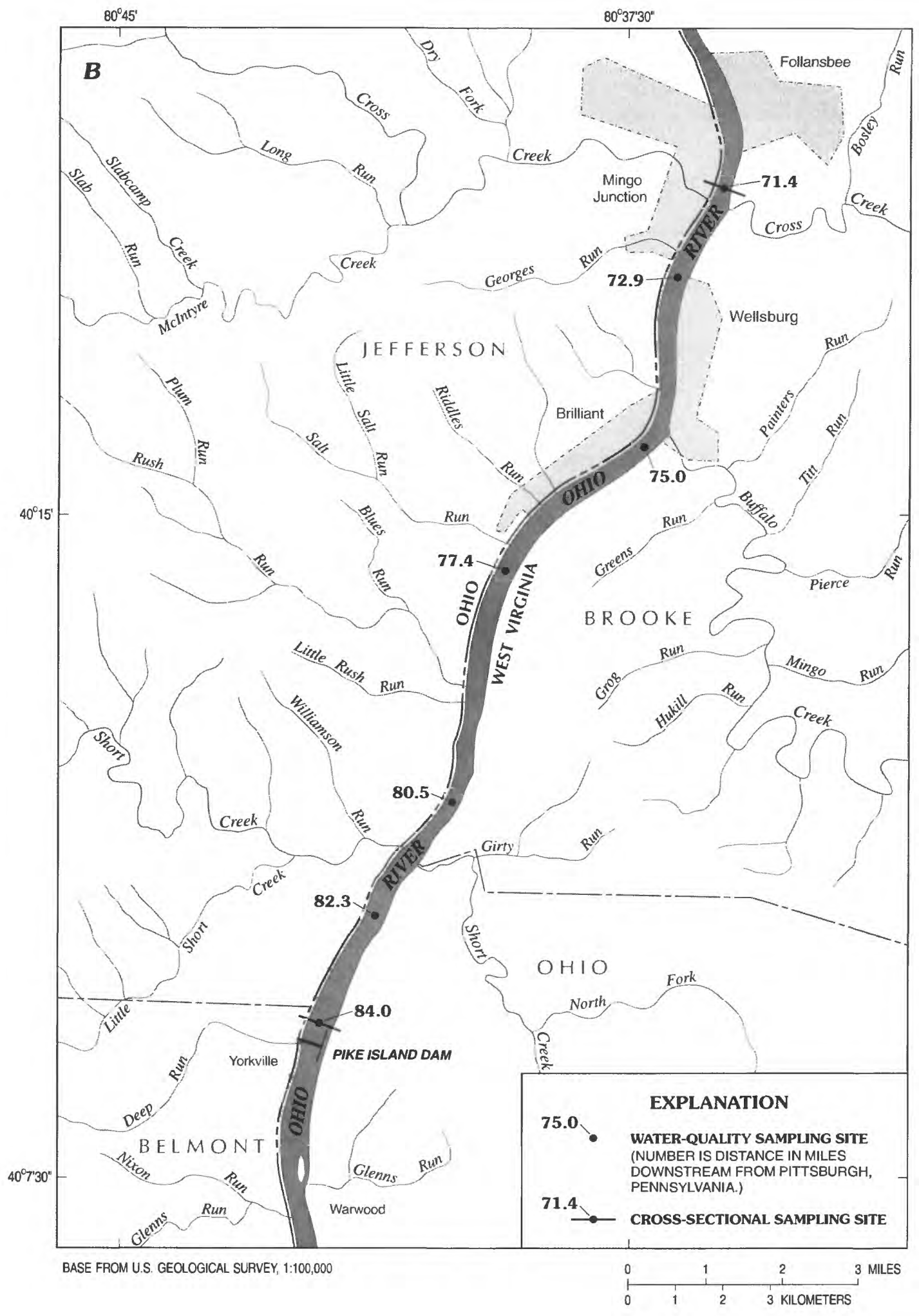

Figure 3b. Water-quality and cross-sectional sampling sites in the downstream section of the study reach. 
water until the disk was no longer visible from the surface, and recording the depth. All Secchi disk measurements were made between the hours of 1000 and 1600 Eastern Daylight Savings Time (EDT). Secchi-disk depths were not recorded if the sampling time was outside this time window or if high surface waves made accurate measurement impossible.

\section{Continuous-Record Water-Quality Monitoring}

Continuous-recording water-quality monitors were installed in June 1995 at sites upstream and downstream from New Cumberland Dam (fig. 4). Upstream, a Hydrolab Datasonde 3 multiparameter data transmitter recorded hourly measurements of specific conductance, $\mathrm{pH}$, water temperature, and DO concentration. The upstream monitor was housed in a section of 6-in. polyvinyl chloride (PVC) pipe at a fixed depth of $6.6 \mathrm{ft}$ and suspended from a United States Coast Guard buoy located approximately in the middle of the navigation channel (latitude $40^{\circ} 31^{\prime} 55^{\prime \prime} \mathrm{N}$., longitude $80^{\circ} 37^{\prime} 35^{\prime \prime} \mathrm{W}$.,). Downstream, a Hydro-lab $\mathrm{H}^{2} 0$ multiparameter data transmitter connected to a Handar 570A data-collection platform recorded hourly values of specific conductance, $\mathrm{pH}$, water temperature, and DO concentration, and transmitted data at 4-hour intervals by way of the Geostationary Operational Environmental Satellite (GOES). The downstream monitor was housed in

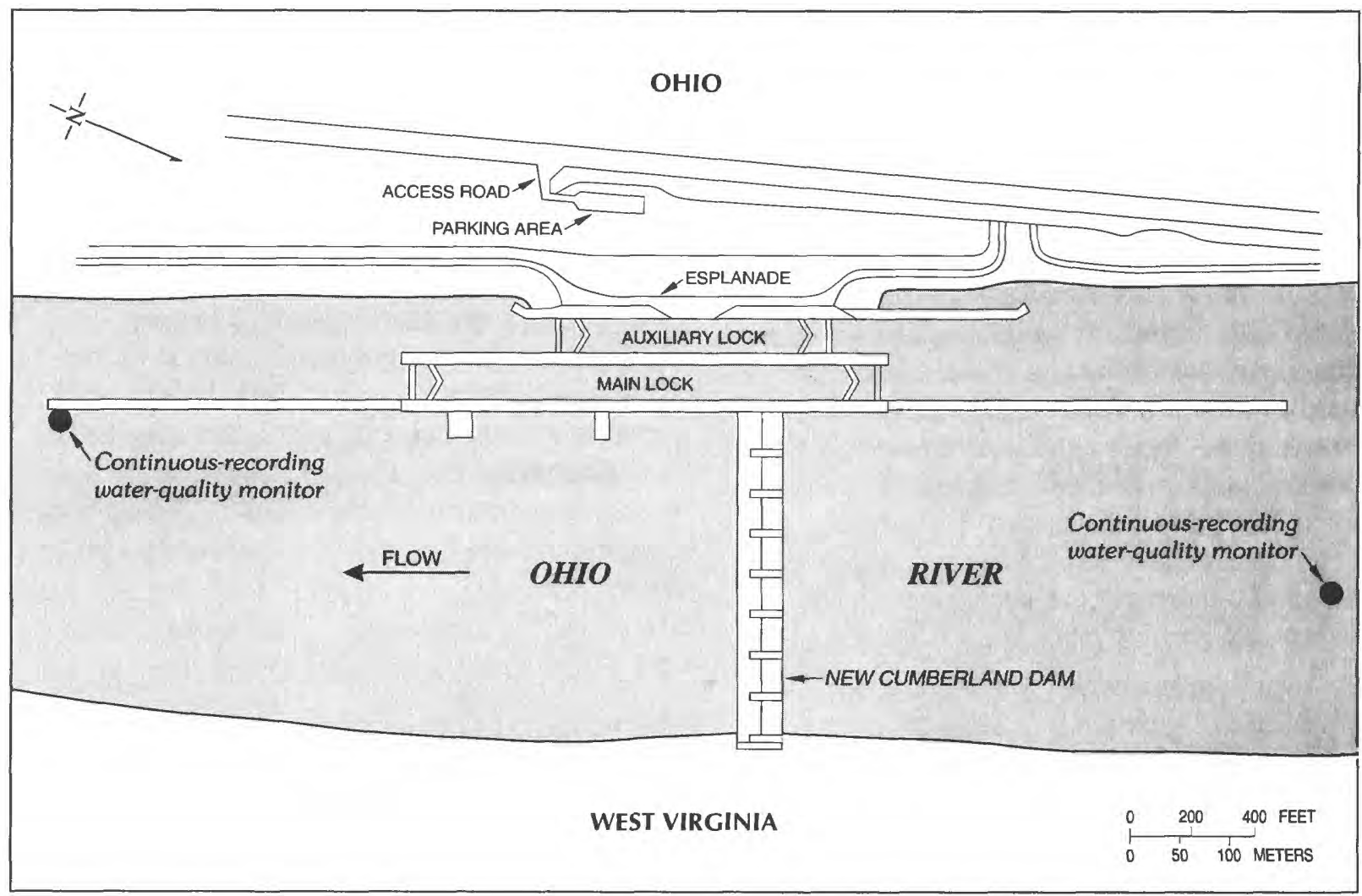

Figure 4. Schematic diagram of New Cumberland Dam showing location of continuous-recording water-quality monitors. 
a section of PVC pipe 6 in. in diameter. It was mounted at a fixed depth of $6.6 \mathrm{ft}$ below the surface of the water and was located at the end of the downstream wingwall on the riverside, about $1,200 \mathrm{ft}$ from the dam (latitude $40^{\circ} 31^{\prime} 33^{\prime \prime} \mathrm{N}$, longitude $80^{\circ} 37^{\prime} 28^{\prime \prime} \mathrm{W}$ ).

\section{Quality Assurance}

The portable water-quality monitoring system was calibrated at the beginning of each sampling period in accordance with the recommendations of the manufacturer (Hydrolab Corporation, 1991), and each parameter was checked periodically during the day for meter drift. Barometric pressure was recorded before each set of field measurement by use of an analog barometer that was calibrated against a mercury barometer maintained by the National Weather Service Forecast Office in Charleston, W. Va.

The portable monitoring system measures DO concentration electrometrically with a standard membrane electrode. The electrode was calibrated by reading the meter against water-saturated air at known temperature and barometric pressure. As a further check of the accuracy of the DO concentration measurements, the electrode response was tested with a solution of sodium sulfite of sufficient concentration (about $1 \mathrm{~g} / \mathrm{L}$ ) to reduce DO concentration to below the detection limit $(0.2 \mathrm{mg} / \mathrm{L})$ of the meter (Skougstad and others, 1979).

At least once during each set of crosssectional transect measurements, a water sample was collected from a point in the cross section at the same time that electrode measurements were recorded, and the DO concentration of the water sample was determined immediately by the Winkler method with azide modification (American Public Health Association and others, 1992, p. 4-100). The meter response was considered accurate if it differed from the results of the Winkler test by no more than $0.2 \mathrm{mg} / \mathrm{L}$. Differences of less than $0.2 \mathrm{mg} / \mathrm{L}$ in reported DO concentrations probably are not significant. DO concentration, as a percentage of the saturation concentration, was calculated using the equations and tables of Weiss (1970).
Secchi disk measurements were made by the same individual between the hours of 1000 and 1600 EDT. Secchi disk depths were not recorded if the sampling time was outside this time frame or if high flows or surface waves made measuring impossible.

The continuous-recording water-quality monitors were serviced and recalibrated according to the manufacturer's instructions at least once every 2 weeks, and more frequently during periods of high water temperatures and low riverflows. Two sensor packages were available for each monitoring location so that a precalibrated unit could be installed at a site and the existing unit removed and returned to the laboratory for servicing. Data from the downstream monitor were transmitted from the Data Collection Platform (DCP) by way of the GOES satellite to a local read-out ground station and from there by way of Internet to the Prime. After being transmitted to the Prime, it was processed through Device Conversion \& Delivery System (DECODES) and loaded into standard data format into the Automated Data Processing System (ADAPS). Occasionally, there were interruptions to the satellite transmissions and the process was altered. Amendments to the process included downloading the data to a disk and manually processing it through DECODES. Data from the upstream monitor were downloaded to a disk and manually processed through DECODES. Personnel from the USGS West Virginia District performed daily quality control by scanning the unedited data, and checking for data interruptions and erroneous values. Raw data were checked for meter drift and corrected, when necessary, by assuming a linear rate of change between successive recalibrations.

\section{WATER-QUALITY DATA}

Water-quality data collected in the Ohio River from New Cumberland Dam to Pike Island Dam during June through October 1995 are presented in tables 1 to 29. Data for the cross-sectional and longitudinal transects are presented in tables 1 to 21. The data are arranged according to location of sampling, date, and depth of sampling. Summaries of continuously recorded water-quality data are presented in tables 22 through 29 and are arranged 
according to date, parameter sampled, and location of sample.

\section{Cross-Sectional and Longitudinal - Transect Data}

Tables 1 through 21 present water-quality data for cross-sectional and longitudinal transects. Each table contains all water-quality data collected during 1995 at the sampling point indicated. Sampling points are identified by station number and by river mile. The main shipping channel in the Pike Island pool is to the left (the West Virginia side) of Browns Island, the largest island in the pool; the back channel is to the right (the Ohio side) of the island. In this report, locations for both main-channel and back-channel sampling sites are always given as the total distance from the left bank to the middle of the channel. Data are stored electronically in the U.S. Geological Survey Water Data Storage and Retrieval System (WATSTORE). At locations where crosssectional-transect data were collected, the location of each depth profile is given as the estimated distance in feet from the left bank of the river and the sampling depth is given in feet below the surface of the water.

Complete sets of data were collected for the July 11, August 8, August 22, and September 7-8 sampling periods. Because of weather conditions, incomplete data sets were collected for the June 27, July 25, September 22, October 3, and October 17 sampling periods.
Secchi disk transparency is a measure of the relative amount of light available for photosynthesis (Wetzel and Likens, 1979). The depth at which the Secchi disk disappears from view is affected by the concentration of suspended particles and by light-absorbing characteristics of the water. No Secchi disk data were reported before the hour of 1000 nor after the hour of 1600 EDT. Weather conditions and high flows on the river occasionally precluded the collection of Secchi disk data.

\section{Continuous-Record Water-quality Monitoring Data}

Continuously recorded monitored waterquality data for the Ohio River at the New Cumberland Dam from June through October 1995 are summarized in tables 22 through 29 . These tables contain daily maximum, minimum, and mean values for specific conductance, water temperature, and DO concentration and daily maximum, minimum, and median values for $\mathrm{pH}$ for both upstream and downstream continuousrecording monitors. The locations of the monitors are identified by station number and as either the upstream or the downstream location; monitor locations are shown in figure 4 . If less than 80 percent of hourly values were recorded for a day, a mean value was not reported for that day. Hourly records are stored permanently in the USGS National Water Information System (NWIS) data base. 


\section{SUMMARY}

The water-quality data presented in this report were collected during the summer and fall of 1995 as part of a monitoring program designed to assess the effects of hydropower development on water quality in the Pike Island navigation pool of the Ohio River (Ohio River miles 51.1 to 84.0). The data were collected, in part, to satisfy license requirements for development of hydropower at New Cumberland Dam (FERC Hydroelectric Project No. 6901).

Data-collection methods consisted of repeated synoptic sampling of selected water-quality characteristics throughout the pool and continuousrecord monitoring.

During synoptic sampling, specific conductance, $\mathrm{pH}$, water temperature, and dissolved oxygen concentration were measured along a longitudinal transect of 20 mid-channel sampling sites. Water-quality measurements also were made at two sites located on the back-channel (Ohio) side of Browns Island and at one site near the middle of the wingwall of New Cumberland Dam. Longitudinal-transect and back-channel sites were sampled in the middle of the channel at the surface, about $3.0 \mathrm{ft}$ below the surface, at the middle of the water column, and near the bottom. Crosssectional transects of the same water-quality measurements were made at 5 of the 20 mainchannel sites. Cross-sectional transects consisted of three to four vertical profiles with measurements at the surface, $3.0 \mathrm{ft}, 5.0 \mathrm{ft}$, and then at intervals of $5.0 \mathrm{ft}$. An estimate of the depth of light penetration (Secchi disk depth) was made at each crosssectional sampling site whenever light and riversurface conditions were appropriate. Synoptic water-quality measurements were made twice in July, August, September, and October 1995. A partial data set was collected in June 1995.

Continuous-recording water-quality monitors were installed immediately upstream and downstream from the New Cumberland Dam. Hourly measurements of specific conductance, $\mathrm{pH}$, water temperature, and dissolved oxygen concentration were recorded beginning in June and continued through October 1995. Maximum, minimum, and mean daily values of specific conductance, water temperature, and dissolved oxygen concentration are reported. Maximum, minimum, and median daily values of $\mathrm{pH}$ are reported. 
American Public Health Association, American Water Works Association, and Water Pollution Control Association, 1992, Standard methods for the examination of water and wastewater, 18th ed.: Washington, D.C., p. 4-100.

Avery, S.T., and Novak, Pavel, 1978, Oxygen transfer at hydraulic structures: Journal of the Hydraulics Division, Proceedings of the American Society of Civil Engineers, v. 104, no. HY11, p. 1521-1540.

Daniil, E.I., Gulliver, J.S., and Thene, J.R., 1991, Water-quality impact assessment for hydropower: Journal of Environmental Engineering, v. 117, no. 2, p. 179-192.

Federal Energy Regulatory Commission, 1988, Hydroelectric development in the upper Ohio River basin--Final environmental impact statement, FERC Docket No. EL85-19-114: Washington, D.C., Federal Energy Regulatory Commission, Office of Hydropower Licensing [variously paged].

Gulliver, J.S., Daniil, E.I., and Thene, J.R., 1990 , Assessing hydro projects' effect on DO concentration: Hydro-Review, v. 9, no. 6, p. 62-69.

Hydrolab Corporation, 1991, Surveyor 3. Multiparameter water quality logging system. Operating manual: Austin, Tex., Hydrolab Corporation, $87 \mathrm{p}$.
Ohio River Valley Water Sanitation

Commission, 1988, Ohio River water quality fact book 1988: Cincinnati, Ohio, Ohio River Valley Water Sanitation Commission, 157 p.

Skougstad, M.W., and others, 1979, Methods for determination of inorganic substances in water and fluvial sediments: U.S. Geological Survey Techniques of Water-Resources Investigations, book 5, chap. A1, p. 537-544.

U.S. Army Corps of Engineers, 1990, Ohio River navigation system, 1990: Cincinnati, Ohio, U.S. Army Corps of Engineers, Ohio River Division, $55 \mathrm{p}$.

Weiss, R.F., 1970, The solubility of nitrogen, oxygen and argon in water and seawater: Deep Sea Research, v. 17, no. 4, p. 721-735.

West Virginia Department of Natural Resources, 1988, Ohio River basin plan: Charleston, W. Va., Department of Natural Resources. $350 \mathrm{p}$. 1989, West Virginia water quality status assessment, 1987-1989: Charleston, W. Va., West Virginia Department of Natural Resources, $131 \mathrm{p}$.

Wetzel, R.G., and Likens, G.E., 1979, Limnological analysis: Philadelphia, W.B. Saunders Company, $357 \mathrm{p}$. 
Table 1. Water-quality data for station 403400080392201, Ohio River at river mile 51.1, June to October, 1995.

ft $=$ feet $; \mu \mathrm{S} / \mathrm{cm}=$ microsiemens per centimeter; ${ }^{\circ} \mathrm{C}=$ degrees Celsius:

$\mathrm{mg} / \mathrm{L}=\mathrm{milligrams}$ per liter; . = data not collected]

\begin{tabular}{|c|c|c|c|c|c|c|c|c|c|}
\hline Date & Time & $\begin{array}{l}\text { Sampling } \\
\text { depth } \\
\text { (ft) }\end{array}$ & $\begin{array}{c}\text { Sample } \\
\text { location } \\
\text { (ft from } \\
\text { left bank) }\end{array}$ & $\begin{array}{l}\text { Specific } \\
\text { conduct- } \\
\text { ance } \\
(\mu \mathrm{s} / \mathrm{cm})\end{array}$ & $\begin{array}{l}\text { pH } \\
\text { (stan- } \\
\text { dard } \\
\text { units) }\end{array}$ & $\begin{array}{l}\text { Temper- } \\
\text { ature, } \\
\text { water } \\
\left({ }^{\circ} \mathrm{C}\right)\end{array}$ & $\begin{array}{c}\text { Trans- } \\
\text { parency } \\
\text { (Secchi } \\
\text { disk) } \\
\text { (Et) }\end{array}$ & $\begin{array}{l}\text { Dissolved } \\
\text { oxygen } \\
\text { (mg/L) }\end{array}$ & $\begin{array}{c}\text { Dissolved } \\
\text { oxygen } \\
\text { (percent } \\
\text { satura- } \\
\text { tion) }\end{array}$ \\
\hline
\end{tabular}

Jun

$\begin{array}{rrrr}27 & 1256 & 0.3 & 600 \\ 27 & 1257 & 3.1 & 600 \\ 27 & 1259 & 17 & 600 \\ 27 & 1258 & 33 & 600\end{array}$

600

$600-390$

$600 \quad 394$

$600 \quad 394$

7.4
7.5
7.4
7.4

25.2

- 7.7

96

July

$11 \quad 1130 \quad 0.4 \quad 600$

111130

$11 \quad 1132$

3.1

600

600

600

439

439

439

7.5

25.5

25.4

- 7.4

$\begin{array}{ll}- & 7.1 \\ \text { - } & 7.3\end{array}$

$\begin{array}{lll}11 & 1131 & 48\end{array}$

600

439

7.4

25.3

7.4

24.9

106

$25 \quad 1133$

0.5

600

432

7.4

600

600

434

7.4

27.0

$\begin{array}{ll}7.4 & 27.0\end{array}$

.. 8.4

$\begin{array}{ll}\cdots & 7.8 \\ \cdots & 7.7\end{array}$

96

$25 \quad 1135$

41

431

- 7.1

7.1

$\begin{array}{ll}- & 7.1 \\ \ldots & 7.1\end{array}$

- 7.0

91

600

435

26.9

91

August

$08 \quad 1119$

$08 \quad 1120$

$08 \quad 1121$

0.4
3.0

600

449
448
447
443

477
478
474
474

7.5

28.4

- 7.7

$\begin{array}{ll}7.5 & 28.4 \\ 7.5 & 28.3\end{array}$

$7.5 \quad 28.3$

- 7.6

$\begin{array}{ll}- & 7.6 \\ - & 7.6\end{array}$

101

600

8.2

8.1

29.4

- 7.4

99

$22 \quad 0922$

$22 \quad 0923$

0.7
3.0
25

600

600

600

7.7

29.3

28.7

- 8.5

. 8.5

$\ldots$

- $\quad 7.4$

113

47

September

$07 \quad 1858$

$07 \quad 1858$

$07 \quad 1900$

$07 \quad 1859$

0.9
2.8
20
40

600

475

8.8

28.6

$\begin{array}{ll}8.8 & 28.5\end{array}$

- 10.0

132

600

600

476

492

8.1

28.5

-. 10.0

10.0

-
$\ldots \quad 7.7$
$\ldots$

132

$8.0 \quad 26.1$

$\cdots \quad 7.5$

95

$22 \quad 1449$

$22 \quad 1450$

0.9

600

487

7.4

22.1

- 7.2

$7.4 \quad 22.1$

$\begin{array}{ll}-. & 7.2 \\ - & 7.2\end{array}$

- 7.1

84

486

7.4

22
44

600

487

600

600

600

486

485

7.5

489

7.5

20.5

$\begin{array}{ll}7.5 & 20.4 \\ 7.5 & 20.3\end{array}$

-

8.3

8.3

$-$

8.2

8.1

94

489

$7.5 \quad 20.3$

$-\cdot$ 
Table 1. Water-quality data for station 403400080392201, Ohio River at river mile 51.1, June to October, 1995, Continued.

[ft $=$ feet $; \mu \mathrm{S} / \mathrm{cm}=$ microsiemens per centimeter; ${ }^{\circ} \mathrm{C}=$ degrees Celsius; $\mathrm{mg} / \mathrm{L}=$ milligrams per liter; - - = data not collected]

\begin{tabular}{|c|c|c|c|c|c|c|c|c|c|}
\hline Date & Time & $\begin{array}{l}\text { Sampling } \\
\text { depth } \\
\text { (ft) }\end{array}$ & $\begin{array}{c}\text { Sample } \\
\text { location } \\
\text { (ft from } \\
\text { left bank) }\end{array}$ & $\begin{array}{l}\text { Specific } \\
\text { conduct- } \\
\text { ance } \\
(\mu \mathrm{S} / \mathrm{cm})\end{array}$ & $\begin{array}{l}\text { pH } \\
\text { (stan- } \\
\text { dard } \\
\text { units) }\end{array}$ & $\begin{array}{c}\text { Temper- } \\
\text { ature, } \\
\text { water } \\
\left({ }^{\circ} \mathrm{C}\right)\end{array}$ & $\begin{array}{l}\text { Trans- } \\
\text { parency } \\
\text { (Secchi } \\
\text { disk) } \\
\text { (ft) }\end{array}$ & $\begin{array}{l}\text { Dissolved } \\
\text { oxygen } \\
(\mathrm{mg} / \mathrm{L})\end{array}$ & $\begin{array}{c}\text { Dissolved } \\
\text { oxygen } \\
\text { (percent } \\
\text { satura- } \\
\text { tion) }\end{array}$ \\
\hline \multicolumn{10}{|c|}{ October } \\
\hline $\begin{array}{l}17 \\
17 \\
17 \\
17\end{array}$ & $\begin{array}{l}1430 \\
1430 \\
1432 \\
1431\end{array}$ & $\begin{array}{l}1 \cdot 1 \\
3 \cdot 1 \\
23 \\
45\end{array}$ & $\begin{array}{l}600 \\
600 \\
600 \\
600\end{array}$ & $\begin{array}{l}459 \\
460 \\
461 \\
458\end{array}$ & $\begin{array}{l}7.5 \\
7.5 \\
7.5 \\
7.5\end{array}$ & $\begin{array}{l}17.8 \\
17.8 \\
17.4 \\
17.4\end{array}$ & $\begin{array}{l}-- \\
\cdots \\
-- \\
--\end{array}$ & $\begin{array}{l}8.6 \\
8.6 \\
8.4 \\
8.4\end{array}$ & $\begin{array}{l}91 \\
91 \\
89 \\
88\end{array}$ \\
\hline
\end{tabular}


Table 2. Water-quality data for station 403156080373201, Ohio River at river mile 54.0, June to October, 1995.

[ft $=$ feet; $\mu \mathrm{S} / \mathrm{cm}=$ microsiemens per centimeter $;{ }^{\circ} \mathrm{C}=$ degrees Celsius; $\mathrm{mg} / \mathrm{L}=$ milligrams pex liter; - - = data not collected]

\begin{tabular}{|c|c|c|c|c|c|c|c|c|c|}
\hline Date & Time & $\begin{array}{l}\text { Sampling } \\
\text { depth } \\
\text { (ft) }\end{array}$ & $\begin{array}{c}\text { Sample } \\
\text { location } \\
\text { (Et from } \\
\text { left bank) }\end{array}$ & $\begin{array}{l}\text { Specific } \\
\text { conduct - } \\
\text { ance } \\
(\mu \mathrm{s} / \mathrm{cm})\end{array}$ & $\begin{array}{l}\mathrm{pH} \\
\text { (stan- } \\
\text { dard } \\
\text { units) }\end{array}$ & $\begin{array}{c}\text { Temper- } \\
\text { ature, } \\
\text { water } \\
\left({ }^{\circ} \mathrm{C}\right)\end{array}$ & $\begin{array}{l}\text { Trans- } \\
\text { parency } \\
\text { (secchi } \\
\text { disk) } \\
\text { (ft) }\end{array}$ & $\begin{array}{l}\text { Dissolved } \\
\text { oxygen } \\
(\mathrm{mg} / \mathrm{L})\end{array}$ & $\begin{array}{c}\text { Dissolved } \\
\text { oxygen } \\
\text { (pexcent } \\
\text { satura- } \\
\text { tion) }\end{array}$ \\
\hline
\end{tabular}

\begin{tabular}{|c|c|c|c|c|c|c|c|c|c|}
\hline \multicolumn{10}{|l|}{ June } \\
\hline 27 & 1355 & 0.6 & 500 & 381 & 7.5 & 28.2 & -- & 7.2 & 94 \\
\hline 27 & 1355 & 3.0 & 500 & 379 & 7.5 & 26.8 & -- & 7.1 & 91 \\
\hline 27 & 1356 & 4.7 & 500 & 380 & 7.5 & 26.3 & $\cdots$ & 7.1 & 90 \\
\hline 27 & 1356 & 9.7 & 500 & 378 & 7.5 & 25.6 & -- & 7.1 & 88 \\
\hline 27 & 1357 & 15 & 500 & 382 & 7.5 & 25.4 & -- & 7.0 & 87 \\
\hline 27 & 1357 & 20 & 500 & 385 & 7.5 & 25.2 & -- & 7.0 & 87 \\
\hline 27 & 1358 & 25 & 500 & 386 & 7.5 & 25.2 & - & 7.0 & 87 \\
\hline 27 & 1359 & 28 & 500 & 381 & 7.5 & 25.2 & - & 6.9 & 86 \\
\hline 27 & 1329 & 0.5 & 900 & 381 & 7.5 & 25.6 & - - & 7.2 & 90 \\
\hline 27 & 1329 & 3.1 & 900 & 382 & 7.5 & 25.5 & 1.5 & 7.2 & 90 \\
\hline 27 & 1330 & 5.0 & 900 & 384 & 7.5 & 25.4 & - & 7.2 & 89 \\
\hline 27 & 1331 & 9.9 & 900 & 382 & 7.5 & 25.2 & -- & 7.0 & 87 \\
\hline 27 & 1331 & 15 & 900 & 380 & 7.5 & 25.2 & -- & 7.0 & 87 \\
\hline 27 & 1332 & 20 & 900 & 381 & 7.4 & 25.2 & -- & 7.0 & 86 \\
\hline 27 & 1332 & 25 & 900 & 384 & 7.4 & 25.2 & - & 6.9 & 86 \\
\hline 27 & 1333 & 30 & 900 & 382 & 7.4 & 25.2 & $\cdots$ & 6.9 & 86 \\
\hline 27 & 1334 & 35 & 900 & 382 & 7.4 & 25.1 & -- & 6.9 & 86 \\
\hline 27 & 1343 & 0.6 & 1,400 & 381 & 7.5 & 26.5 & $\cdots$ & 7.2 & 92 \\
\hline 27 & 1343 & 3.2 & 1,400 & 383 & 7.5 & 26.5 & -- & 7.2 & 92 \\
\hline 27 & 1344 & 5.2 & 1,400 & 384 & 7.5 & 26.1 & -- & 7.2 & 91 \\
\hline 27 & 1345 & 10 & 1,400 & 386 & 7.5 & 25.5 & $\cdots$ & 7.2 & 90 \\
\hline 27 & 1346 & 15 & 1,400 & 388 & 7.5 & 25.2 & -- & 7.1 & 88 \\
\hline 27 & 1346 & 20 & 1,400 & 385 & 7.5 & 25.2 & $\cdots$ & 7.0 & 86 \\
\hline 27 & 1347 & 25 & 1,400 & 382 & 7.5 & 25.2 & $\cdots$ & 6.9 & 86 \\
\hline 27 & 1348 & 30 & 1,400 & 386 & 7.5 & 25.2 & $\cdots$ & 6.9 & 85 \\
\hline 27 & 1349 & 33 & 1,400 & 382 & 7.4 & 25.2 & $\cdots$ & 6.8 & \\
\hline \multicolumn{10}{|l|}{ July } \\
\hline 11 & 1104 & 0.3 & 500 & 428 & 7.4 & 29.5 & - & 7.4 & 99 \\
\hline 11 & 1104 & 3.1 & 500 & 428 & 7.4 & 29.2 & $\cdots$ & 7.5 & 100 \\
\hline 11 & 1105 & 5.0 & 500 & 427 & 7.4 & 28.9 & -- & 7.5 & 99 \\
\hline 11 & 1105 & 9.9 & 500 & 426 & 7.3 & 27.7 & -- & 7.4 & 96 \\
\hline 11 & 1106 & 15 & 500 & 426 & 7.3 & 26.2 & $\cdots$ & 7.5 & 9 \\
\hline 11 & 1107 & 20 & 500 & 426 & 7.3 & 25.8 & $\cdots$ & 7.4 & 9 \\
\hline 11 & 1107 & 25 & 500 & 429 & 7.3 & 25.2 & -- & 7.2 & 9 \\
\hline 11 & 1108 & 29 & 500 & 432 & 7.3 & 25.1 & $\cdots$ & 7.2 & 89 \\
\hline
\end{tabular}


Table 2. Water-quality data for station 403156080373201, Ohio River at river mile 54.0, June to October, 1995, Continued.

[ft = feet; $\mu \mathrm{s} / \mathrm{cm}=$ microsiemens per centimeter $;{ }^{\circ} \mathrm{C}=$ degrees Celsius;

$\mathrm{mg} / \mathrm{L}=\mathrm{mili}$ igrams per liter; - = data not collected]

\begin{tabular}{|c|c|c|c|c|c|c|c|c|c|}
\hline Date & Time & $\begin{array}{l}\text { Sampling } \\
\text { depth } \\
\text { (ft) }\end{array}$ & $\begin{array}{c}\text { Sample } \\
\text { location } \\
\text { (ft from } \\
\text { left bank) }\end{array}$ & $\begin{array}{l}\text { Specific } \\
\text { conduct- } \\
\text { ance } \\
(\mu S / \mathrm{cm})\end{array}$ & $\begin{array}{l}\text { pH } \\
\text { (stan- } \\
\text { dard } \\
\text { units) }\end{array}$ & $\begin{array}{c}\text { Temper- } \\
\text { ature, } \\
\text { water } \\
\left({ }^{\circ} \mathrm{C}\right)\end{array}$ & $\begin{array}{l}\text { Trans - } \\
\text { parency } \\
\text { (secchi } \\
\text { disk) } \\
\text { (ft) }\end{array}$ & $\begin{array}{l}\text { Dissolved } \\
\text { oxygen } \\
(\mathrm{mg} / \mathrm{L})\end{array}$ & $\begin{array}{c}\text { Dissolved } \\
\text { oxygen } \\
\text { (percent } \\
\text { satura- } \\
\text { tion) }\end{array}$ \\
\hline
\end{tabular}

\begin{tabular}{|c|c|c|c|c|c|c|c|c|c|}
\hline $\begin{array}{r}\text { July } \\
11 \\
11 \\
11 \\
11 \\
11 \\
11 \\
11 \\
11 \\
11\end{array}$ & $\begin{array}{l}1055 \\
1055 \\
1056 \\
1056 \\
1057 \\
1057 \\
1058 \\
1058 \\
1059\end{array}$ & $\begin{array}{c}0.4 \\
3.1 \\
5.0 \\
10 \\
15 \\
20 \\
25 \\
30 \\
33\end{array}$ & $\begin{array}{lll}9 & 0 & 0 \\
9 & 0 & 0 \\
9 & 0 & 0 \\
9 & 0 & 0 \\
9 & 0 & 0 \\
9 & 00 \\
9 & 0 & 0 \\
9 & 0 & 0 \\
9 & 0 & 0\end{array}$ & $\begin{array}{l}425 \\
424 \\
432 \\
423 \\
434 \\
436 \\
434 \\
425 \\
424\end{array}$ & $\begin{array}{l}7.3 \\
7.3 \\
7.3 \\
7.3 \\
7.3 \\
7.3 \\
7.3 \\
7.3 \\
7.3\end{array}$ & $\begin{array}{l}29.4 \\
28.9 \\
28.5 \\
27.6 \\
26.0 \\
25.5 \\
25.2 \\
25.1 \\
25.1\end{array}$ & $\begin{array}{r}- \\
2.5 \\
- \\
- \\
- \\
- \\
- \\
- \\
-\end{array}$ & $\begin{array}{l}7.3 \\
7.4 \\
7.4 \\
7.3 \\
7.4 \\
7.4 \\
7.4 \\
7.3 \\
7.3\end{array}$ & $\begin{array}{l}98 \\
98 \\
97 \\
95 \\
94 \\
93 \\
91 \\
91 \\
90\end{array}$ \\
\hline $\begin{array}{l}11 \\
11 \\
11 \\
11 \\
11 \\
11 \\
11 \\
11\end{array}$ & $\begin{array}{l}1110 \\
1110 \\
1111 \\
1111 \\
1112 \\
1112 \\
1113 \\
1113\end{array}$ & $\begin{array}{l}0.2 \\
3.0 \\
5.1 \\
10 \\
15 \\
20 \\
25 \\
29\end{array}$ & $\begin{array}{l}1,400 \\
1,400 \\
1,400 \\
1,400 \\
1,400 \\
1,400 \\
1,400 \\
1,400\end{array}$ & $\begin{array}{l}429 \\
428 \\
427 \\
428 \\
427 \\
431 \\
430 \\
424\end{array}$ & $\begin{array}{l}7.3 \\
7.3 \\
7.3 \\
7.3 \\
7.3 \\
7.3 \\
7.3 \\
7.3\end{array}$ & $\begin{array}{l}29.5 \\
29.0 \\
28.2 \\
26.7 \\
26.3 \\
25.6 \\
25.4 \\
25.3\end{array}$ & $\begin{array}{l}- \\
- \\
- \\
- \\
- \\
- \\
- \\
-\end{array}$ & $\begin{array}{l}7.4 \\
7.3 \\
7.4 \\
7.4 \\
7.4 \\
7.3 \\
7.3 \\
7.0\end{array}$ & $\begin{array}{l}99 \\
97 \\
96 \\
94 \\
94 \\
91 \\
90 \\
87\end{array}$ \\
\hline $\begin{array}{l}25 \\
25 \\
25 \\
25 \\
25 \\
25 \\
25 \\
25\end{array}$ & $\begin{array}{l}1144 \\
1144 \\
1145 \\
1145 \\
1146 \\
1146 \\
1147 \\
1147\end{array}$ & $\begin{array}{l}0.5 \\
3.1 \\
5.1 \\
10 \\
15 \\
20 \\
25 \\
30\end{array}$ & $\begin{array}{l}500 \\
500 \\
500 \\
500 \\
500 \\
500 \\
500 \\
500\end{array}$ & $\begin{array}{l}427 \\
427 \\
428 \\
427 \\
426 \\
426 \\
426 \\
428\end{array}$ & $\begin{array}{l}7.4 \\
7.4 \\
7.4 \\
7.4 \\
7.4 \\
7.4 \\
7.4 \\
7.4\end{array}$ & $\begin{array}{l}32.2 \\
32.1 \\
32.0 \\
30.3 \\
29.2 \\
28.1 \\
27.3 \\
27.2\end{array}$ & $\begin{array}{l}-- \\
-- \\
-- \\
-- \\
-- \\
--\end{array}$ & $\begin{array}{l}6.9 \\
7.0 \\
6.9 \\
6.8 \\
6.8 \\
6.8 \\
6.5 \\
6.4\end{array}$ & $\begin{array}{l}97 \\
98 \\
96 \\
92 \\
90 \\
89 \\
84 \\
83\end{array}$ \\
\hline $\begin{array}{l}25 \\
25 \\
25 \\
25 \\
25 \\
25 \\
25 \\
25 \\
25\end{array}$ & $\begin{array}{l}1214 \\
1214 \\
1215 \\
1215 \\
1216 \\
1216 \\
1217 \\
1217 \\
1218\end{array}$ & $\begin{array}{c}0.3 \\
3.1 \\
5.1 \\
10 \\
15 \\
20 \\
25 \\
30 \\
35\end{array}$ & $\begin{array}{lll}9 & 0 & 0 \\
9 & 0 & 0 \\
9 & 0 & 0 \\
9 & 0 & 0 \\
9 & 0 & 0 \\
9 & 0 & 0 \\
9 & 0 & 0 \\
9 & 0 & 0 \\
9 & 0 & 0\end{array}$ & $\begin{array}{l}427 \\
426 \\
425 \\
427 \\
426 \\
424 \\
422 \\
422 \\
430\end{array}$ & $\begin{array}{l}7.4 \\
7.4 \\
7.4 \\
7.4 \\
7.4 \\
7.4 \\
7.4 \\
7.4 \\
7.4\end{array}$ & $\begin{array}{l}31.7 \\
31.7 \\
31.7 \\
30.0 \\
28.3 \\
27.5 \\
27.3 \\
27.3 \\
27.3\end{array}$ & $\begin{array}{r}\cdots \\
2.5 \\
- \\
- \\
- \\
- \\
- \\
- \\
-\end{array}$ & $\begin{array}{l}6.9 \\
6.9 \\
6.9 \\
6.8 \\
6.9 \\
6.8 \\
6.7 \\
6.7 \\
6.6\end{array}$ & $\begin{array}{l}96 \\
95 \\
96 \\
92 \\
90 \\
88 \\
87 \\
87 \\
86\end{array}$ \\
\hline
\end{tabular}


Table 2. Water-quality data for station 403156080373201 , Ohio River at river mile 54.0, June to October, 1995, Continued.

[ft = feet; $\mu \mathrm{s} / \mathrm{cm}=$ microsiemens per centimeter; ${ }^{\circ} \mathrm{C}=$ degrees Celsius;

$\mathrm{mg} / \mathrm{L}=\mathrm{milligrams}$ per liter; $\cdots=$ data not collected]

\begin{tabular}{|c|c|c|c|c|c|c|c|c|c|}
\hline Date & Time & $\begin{array}{l}\text { Sampling } \\
\text { depth } \\
\text { (ft) }\end{array}$ & $\begin{array}{c}\text { Sample } \\
\text { location } \\
\text { (ft from } \\
\text { left bank) }\end{array}$ & $\begin{array}{l}\text { specific } \\
\text { conduct- } \\
\text { ance } \\
(\mu \mathrm{S} / \mathrm{cm})\end{array}$ & $\begin{array}{l}\text { pH } \\
\text { (stan- } \\
\text { dard } \\
\text { units) }\end{array}$ & $\begin{array}{c}\text { Temper- } \\
\text { ature, } \\
\text { water } \\
\left({ }^{\circ} \mathrm{C}\right)\end{array}$ & $\begin{array}{l}\text { Trans- } \\
\text { parency } \\
\text { (Secchi } \\
\text { disk) } \\
\text { (ft) }\end{array}$ & $\begin{array}{l}\text { Dissolved } \\
\text { oxygen } \\
(\mathrm{mg} / \mathrm{L})\end{array}$ & $\begin{array}{c}\text { Dissolved } \\
\text { oxygen } \\
\text { (percent } \\
\text { satura- } \\
\text { tion) }\end{array}$ \\
\hline
\end{tabular}

$\begin{array}{rr}\text { July } & \\ 25 & 1152 \\ 25 & 1152 \\ 25 & 1153 \\ 25 & 1153 \\ 25 & 1154 \\ 25 & 1154 \\ 25 & 1155 \\ 25 & 1155\end{array}$

$\begin{array}{rr}0.6 & 1,400 \\ 3.2 & 1,400 \\ 5.0 & 1,400 \\ 10 & 1,400 \\ 15 & 1,400 \\ 20 & 1,400 \\ 25 & 1,400 \\ 27 & 1,400\end{array}$

$\begin{array}{ll}428 & 7.4 \\ 427 & 7.4 \\ 425 & 7.4 \\ 427 & 7.4 \\ 427 & 7.4 \\ 427 & 7.4 \\ 429 & 7.4 \\ 423 & 7.4\end{array}$

32.5
31.7
30.8
29.6
28.8
27.8
27.4
27.3

$-$

6.8

$\quad 6.8$

$-$

$\begin{array}{ll}-. & 6.8 \\ \cdots & 6.8\end{array}$

.. 6.7

.. 6.6

-. 6.6

96
94
92
91
90
87
85
85

August

$\begin{array}{ll}08 & 1112 \\ 08 & 1112 \\ 08 & 1113 \\ 08 & 1113 \\ 08 & 1114 \\ 08 & 1114 \\ 08 & 1115 \\ 08 & 1115 \\ 08 & 1116 \\ & \\ 08 & 1100 \\ 08 & 1100 \\ 08 & 1101 \\ 08 & 1101 \\ 08 & 1102 \\ 08 & 1102 \\ 08 & 1103 \\ 08 & 1103 \\ 08 & 1104 \\ & \\ 08 & 1106 \\ 08 & 1106 \\ 08 & 1107 \\ 08 & 1107 \\ 08 & 1108 \\ 08 & 1108 \\ 08 & 1109 \\ 08 & 1109\end{array}$

$0.3 \quad 500$

449

7.5

28.3

- 7.4

$450 \quad 7.5$

$451 \quad 7.5$

$450 \quad 7.5$

$451 \quad 7.5$

$451 \quad 7.4$

$451 \quad 7.4$

449

7.4

28.3

28.3

28.2

28.2

28.2

28.2

28.2

500

$451 \quad 7.4$

28.2

.. 7.3

-. 7.3

-. 7.3

- 7.2

-. 7.2

7.2
.$- \quad 7.2$

- 7.2

- $\quad 7.0$

7.6

449

$449 \quad 7.5$

$449 \quad 7.5$

$450 \quad 7.5$

$450 \quad 7.5$

$451 \quad 7.4$

$452 \quad 7.4$

$450 \quad 7.4$

28.3

28.3

28.3

28.3

28.3

28.3

28.2

28.2

7.4

28.2

- 7.1

-. 7.3

$3.5 \quad 7.1$

$\therefore 7.1$

.. 7.1

-. 7.0

- 7.0

. 7.0

-. 6.9

96

$33 \quad 900$

448

$7.5 \quad 31.1$

449

$\begin{array}{ll}0.4 & 1,400 \\ 3.3 & 1,400\end{array}$

449

451

$10.2 \quad 1,400$

151,400

201,400

25

29

1,400

450

451

449

451

7.5

7.5

7.5

7.5

7.5

7.5

30.6

29.3

28.6

28.5

28.4

28.3

28.3

7.0

$\begin{array}{ll}\cdots & 7.0 \\ \cdots & 7.1\end{array}$

.. 7.1

. 7.0

- 7.0

.. 7.0

.. 7.0

. 6.9

96
95
95
94
94
94
93
92

93
95
92
93
93
91
91
91
89

96
96
94
92
92
92
91
90 
Table 2. Water-quality data for station 403156080373201, Ohio River at river mile 54.0, June to October, 1995, Continued.

[ft $=$ feet $; \mu \mathrm{s} / \mathrm{cm}=$ microsiemens per centimeter; ${ }^{\circ} \mathrm{C}=$ degrees Celsius;

$\mathrm{mg} / \mathrm{L}=\mathrm{mili}$ igrams per liter; $\cdots$ = data not collected

\begin{tabular}{|c|c|c|c|c|c|c|c|c|c|}
\hline Date & Time & $\begin{array}{l}\text { Sampling } \\
\text { depth } \\
\text { (ft) }\end{array}$ & $\begin{array}{c}\text { Sample } \\
\text { location } \\
\text { (ft from } \\
\text { left bank) }\end{array}$ & $\begin{array}{l}\text { Specific } \\
\text { conduct- } \\
\text { ance } \\
(\mu \mathrm{S} / \mathrm{cm})\end{array}$ & $\begin{array}{l}\mathrm{pH} \\
\text { (stan- } \\
\text { dard } \\
\text { units) }\end{array}$ & $\begin{array}{c}\text { Temper- } \\
\text { ature, } \\
\text { water } \\
\left({ }^{\circ} \mathrm{C}\right)\end{array}$ & $\begin{array}{l}\text { Trans- } \\
\text { parency } \\
\text { (Secchi } \\
\text { disk) } \\
\text { (ft) }\end{array}$ & $\begin{array}{l}\text { Dissolved } \\
\text { oxygen } \\
(\mathrm{mg} / \mathrm{L})\end{array}$ & $\begin{array}{c}\text { Dissolved } \\
\text { oxygen } \\
\text { (percent } \\
\text { satura. } \\
\text { tion) }\end{array}$ \\
\hline
\end{tabular}

August

\begin{tabular}{|c|c|c|c|c|c|c|c|c|c|}
\hline $\begin{array}{l}22 \\
22 \\
22 \\
22 \\
22 \\
22 \\
22 \\
22 \\
22\end{array}$ & $\begin{array}{l}0940 \\
0940 \\
0941 \\
0941 \\
0942 \\
0942 \\
0943 \\
0943 \\
0944\end{array}$ & $\begin{array}{l}1.0 \\
3.2 \\
5.2 \\
9.9 \\
15 \\
20 \\
25 \\
30 \\
35\end{array}$ & $\begin{array}{l}500 \\
500 \\
500 \\
500 \\
500 \\
500 \\
500 \\
500 \\
500\end{array}$ & $\begin{array}{l}479 \\
479 \\
483 \\
482 \\
485 \\
482 \\
483 \\
472 \\
488\end{array}$ & $\begin{array}{l}7.6 \\
7.6 \\
7.6 \\
7.6 \\
7.6 \\
7.7 \\
7.6 \\
7.5 \\
7.4\end{array}$ & $\begin{array}{l}34.9 \\
34.9 \\
34.8 \\
34.7 \\
31.6 \\
30.5 \\
29.4 \\
29.2 \\
29.0\end{array}$ & $\begin{array}{l}\cdots \\
\therefore \\
\cdots \\
\cdots \\
\cdots \\
\cdots \\
-\end{array}$ & $\begin{array}{l}7.2 \\
7.1 \\
7.3 \\
7.2 \\
7.2 \\
7.4 \\
6.9 \\
6.5 \\
6.2\end{array}$ & $\begin{array}{r}105 \\
103 \\
106 \\
105 \\
100 \\
100 \\
91 \\
86 \\
82\end{array}$ \\
\hline $\begin{array}{l}22 \\
22 \\
22 \\
22 \\
22 \\
22 \\
22 \\
22 \\
22\end{array}$ & $\begin{array}{l}0953 \\
0953 \\
0954 \\
0954 \\
0955 \\
0955 \\
0956 \\
0956 \\
0957\end{array}$ & $\begin{array}{l}0.6 \\
3.2 \\
5.4 \\
9.8 \\
15 \\
20 \\
25 \\
30 \\
35\end{array}$ & $\begin{array}{l}900 \\
900 \\
900 \\
900 \\
900 \\
900 \\
900 \\
900 \\
900\end{array}$ & $\begin{array}{l}481 \\
480 \\
480 \\
479 \\
483 \\
482 \\
481 \\
476 \\
479\end{array}$ & $\begin{array}{l}7.6 \\
7.6 \\
7.6 \\
7.6 \\
7.6 \\
7.6 \\
7.5 \\
7.5 \\
7.4\end{array}$ & $\begin{array}{l}34.7 \\
34.7 \\
34.7 \\
34.5 \\
31.9 \\
29.5 \\
29.3 \\
29.2 \\
29.0\end{array}$ & $\begin{array}{l}- \\
\cdots \\
\cdots \\
\cdots \\
\cdots \\
- \\
-\end{array}$ & $\begin{array}{l}7.1 \\
7.1 \\
7.0 \\
7.1 \\
7.2 \\
7.1 \\
7.0 \\
6.5 \\
5.7\end{array}$ & $\begin{array}{r}103 \\
103 \\
102 \\
103 \\
99 \\
94 \\
92 \\
86 \\
75\end{array}$ \\
\hline $\begin{array}{l}22 \\
22 \\
22 \\
22 \\
22 \\
22 \\
22\end{array}$ & $\begin{array}{l}1013 \\
1013 \\
1014 \\
1014 \\
1015 \\
1015 \\
1016\end{array}$ & $\begin{array}{c}0.5 \\
3.2 \\
5.2 \\
10 \\
15 \\
20 \\
25\end{array}$ & $\begin{array}{l}1,400 \\
1,400 \\
1,400 \\
1,400 \\
1,400 \\
1,400 \\
1,400\end{array}$ & $\begin{array}{l}479 \\
479 \\
479 \\
482 \\
480 \\
483 \\
478\end{array}$ & $\begin{array}{l}7.6 \\
7.6 \\
7.6 \\
7.6 \\
7.6 \\
7.6 \\
7.5\end{array}$ & $\begin{array}{l}35.3 \\
34.7 \\
34.1 \\
32.6 \\
31.5 \\
30.1 \\
29.3\end{array}$ & $\begin{array}{l}\cdots \\
\cdots \\
\cdots \\
\cdots \\
-\end{array}$ & $\begin{array}{l}7.2 \\
7.1 \\
7.1 \\
7.2 \\
7.2 \\
7.2 \\
6.6\end{array}$ & $\begin{array}{r}105 \\
103 \\
102 \\
101 \\
99 \\
97 \\
88\end{array}$ \\
\hline $\begin{array}{l}\text { epte } \\
07 \\
07 \\
07 \\
07 \\
07 \\
07 \\
07 \\
07\end{array}$ & $\begin{array}{l}\text { nber } \\
1841 \\
1841 \\
1842 \\
1842 \\
1843 \\
1843 \\
1844 \\
1844\end{array}$ & $\begin{array}{c}0.4 \\
2.6 \\
4.8 \\
9.7 \\
15 \\
19 \\
24 \\
30\end{array}$ & $\begin{array}{l}500 \\
500 \\
500 \\
500 \\
500 \\
500 \\
500 \\
500\end{array}$ & $\begin{array}{l}487 \\
485 \\
485 \\
485 \\
482 \\
483 \\
486 \\
490\end{array}$ & $\begin{array}{l}7.9 \\
7.9 \\
7.9 \\
7.8 \\
7.9 \\
7.8 \\
7.8 \\
7.7\end{array}$ & $\begin{array}{l}31.8 \\
31.8 \\
31.8 \\
31.3 \\
29.2 \\
27.3 \\
26.6 \\
26.6\end{array}$ & $\begin{array}{l}- \\
- \\
- \\
- \\
- \\
-\end{array}$ & $\begin{array}{l}7.1 \\
7.2 \\
7.2 \\
7.0 \\
7.2 \\
7.1 \\
6.8 \\
6.7\end{array}$ & $\begin{array}{r}99 \\
100 \\
100 \\
97 \\
96 \\
92 \\
87 \\
85\end{array}$ \\
\hline
\end{tabular}


Table 2. Water-quality data for station 403156080373201, Ohio River at river mile 54.0, June to October, 1995, Continued.

[ft $=$ feet; $\mu \mathrm{S} / \mathrm{cm}=$ microsiemens per centimeter ${ }^{\circ} \mathrm{C}=$ degrees Celsius; $\mathrm{mg} / \mathrm{L}=$ milisgrams per liter; - - = data not collected]

\begin{tabular}{|c|c|c|c|c|c|c|c|c|c|}
\hline Date & Time & $\begin{array}{l}\text { Sampling } \\
\text { depth } \\
\text { (ft) }\end{array}$ & $\begin{array}{c}\text { Sample } \\
\text { location } \\
\text { (Et from } \\
\text { left bank) }\end{array}$ & $\begin{array}{l}\text { Specific } \\
\text { conduct- } \\
\text { ance } \\
(\mu \mathrm{S} / \mathrm{cm})\end{array}$ & $\begin{array}{l}\mathrm{pH} \\
\text { (stan- } \\
\text { dard } \\
\text { units) }\end{array}$ & $\begin{array}{c}\text { Temper- } \\
\text { ature, } \\
\text { water } \\
\left({ }^{\circ} \mathrm{C}\right)\end{array}$ & $\begin{array}{c}\text { Trans - } \\
\text { parency } \\
\text { (secchi } \\
\text { disk) } \\
\text { (ft) }\end{array}$ & $\begin{array}{l}\text { Dissolved } \\
\text { oxygen } \\
\text { (mg/L) }\end{array}$ & $\begin{array}{c}\text { Dissolved } \\
\text { oxygen } \\
\text { (percent } \\
\text { satura- } \\
\text { tion) }\end{array}$ \\
\hline
\end{tabular}

\begin{tabular}{|c|c|c|c|c|c|c|c|c|c|}
\hline \multicolumn{10}{|c|}{ september } \\
\hline 07 & 1834 & 0.2 & 900 & 485 & 7.9 & 31.4 & $\cdots$ & 7.0 & 97 \\
\hline 07 & 1834 & 2.8 & 900 & 484 & 7.9 & 31.5 & $\cdots$ & 7.0 & 97 \\
\hline 07 & 1835 & 4.6 & 900 & 486 & 7.9 & 31.4 & $\cdots$ & 7.0 & 97 \\
\hline 07 & 1835 & 9.4 & 900 & 485 & 7.9 & 31.5 & $\cdots$ & 7.0 & 97 \\
\hline 07 & 1836 & 15 & 900 & 483 & 7.9 & 28.0 & - & 7.3 & 95 \\
\hline 07 & 1836 & 19 & 900 & 486 & 7.8 & 26.8 & - & 7.1 & 90 \\
\hline 07 & 1837 & 25 & 900 & 487 & 7.8 & 26.6 & - & 6.9 & 88 \\
\hline 07 & 1837 & 30 & 900 & 487 & 7.7 & 26.6 & - & 6.8 & 87 \\
\hline 07 & 1838 & 35 & 900 & 487 & 7.7 & 26.6 & - & 6.6 & 84 \\
\hline 07 & 1846 & 0.3 & 1,400 & 486 & 7.9 & 32.0 & - & 7.0 & 99 \\
\hline 07 & 1846 & 2.8 & 1,400 & 486 & 7.8 & 31.6 & - & 7.1 & 98 \\
\hline 07 & 1847 & 4.6 & 1,400 & 484 & 7.9 & 31.4 & $\cdots$ & 7.1 & 99 \\
\hline 07 & 1847 & 9.6 & 1,400 & 484 & 7.9 & 30.0 & - & 7.0 & 94 \\
\hline 07 & 1848 & 15 & 1,400 & 482 & 7.9 & 28.7 & $\cdots$ & 7.0 & 93 \\
\hline 07 & 1848 & 20 & 1,400 & 490 & 7.8 & 26.9 & $\cdots$ & 6.8 & 87 \\
\hline 07 & 1849 & 25 & 1,400 & 491 & 7.7 & 26.6 & $\cdots$ & 6.7 & 86 \\
\hline 07 & 1849 & 28 & 1,400 & 488 & 7.7 & 26.6 & $\cdots$ & 6.5 & 83 \\
\hline 22 & 1437 & 1.1 & 500 & 490 & 7.4 & 25.7 & $\cdots$ & 6.6 & 83 \\
\hline 22 & 1438 & 3.2 & 500 & 491 & 7.4 & 25.7 & $\cdots$ & 6.9 & 86 \\
\hline 22 & 1438 & 5.2 & 500 & 491 & 7.4 & 25.7 & $\cdots$ & 6.8 & 85 \\
\hline 22 & 1439 & 9.8 & 500 & 491 & 7.4 & 24.9 & - & 6.3 & 78 \\
\hline 22 & 1440 & 15 & 500 & 489 & 7.4 & 23.4 & $\cdots$ & 6.9 & 82 \\
\hline 22 & 1440 & 20 & 500 & 490 & 7.4 & 23.2 & - & 6.8 & 81 \\
\hline 22 & 1441 & 25 & 500 & 489 & 7.4 & 22.9 & $\cdots$ & 6.9 & 82 \\
\hline 22 & 1442 & 30 & 500 & 487 & 7.4 & 22.7 & - & 6.5 & 77 \\
\hline 22 & 1429 & 1.0 & 900 & 493 & 7.4 & 25.6 & $\cdots$ & 6.7 & 83 \\
\hline 22 & 1429 & 2.8 & 900 & 492 & 7.4 & 25.7 & $\ldots$ & 6.8 & 85 \\
\hline 22 & 1430 & 4.8 & 900 & 490 & 7.4 & 25.8 & - & 6.8 & 85 \\
\hline 22 & 1430 & 10 & 900 & 493 & 7.4 & 25.2 & $\cdots$ & 6.5 & 81 \\
\hline 22 & 1431 & 15 & 900 & 491 & 7.4 & 23.4 & $\cdots$ & 6.6 & 79 \\
\hline 22 & 1431 & 20 & 900 & 490 & 7.4 & 22.9 & - & 6.8 & 80 \\
\hline 22 & 1432 & 25 & 900 & 494 & 7.4 & 22.8 & $\cdots$ & 7.0 & 82 \\
\hline 22 & 1432 & 30 & 900 & 491 & 7.4 & 22.8 & $\cdots$ & 6.8 & 80 \\
\hline 22 & 1433 & 33 & 900 & 490 & 7.4 & 22.8 & - & 6.5 & 77 \\
\hline
\end{tabular}


Table 2. Water-quality data for station 403156080373201 , Ohio River at river mile 54.0, June to October, 1995, Continued.

[ft $=$ feet; $\mu \mathrm{S} / \mathrm{cm}=$ microsiemens per centimeter; ${ }^{\circ} \mathrm{C}=$ degrees Celsius; $\mathrm{mg} / \mathrm{L}=\mathrm{milligrams}$ per liter; $\cdot-$ = data not collected]

\begin{tabular}{|c|c|c|c|c|c|c|c|c|c|}
\hline Date & Time & $\begin{array}{l}\text { Sampling } \\
\text { depth } \\
\text { (ft) }\end{array}$ & $\begin{array}{c}\text { Sample } \\
\text { location } \\
\text { (ft from } \\
\text { left bank) }\end{array}$ & $\begin{array}{l}\text { specific } \\
\text { conduct- } \\
\text { ance } \\
(\mu \mathrm{s} / \mathrm{cm})\end{array}$ & $\begin{array}{l}\mathrm{pH} \\
\text { (stan- } \\
\text { dard } \\
\text { units) }\end{array}$ & $\begin{array}{c}\text { Temper- } \\
\text { ature, } \\
\text { water } \\
\left({ }^{\circ} \mathrm{C}\right)\end{array}$ & $\begin{array}{l}\text { Trans - } \\
\text { parency } \\
\text { (secchi } \\
\text { disk) } \\
\text { (ft) }\end{array}$ & $\begin{array}{l}\text { Dissolved } \\
\text { oxygen } \\
\text { (mg/L) }\end{array}$ & $\begin{array}{c}\text { Dissolved } \\
\text { oxygen } \\
\text { (percent } \\
\text { satura- } \\
\text { tion) }\end{array}$ \\
\hline
\end{tabular}

$\begin{array}{cc}\text { September } \\ 22 & 1406 \\ 22 & 1406 \\ 22 & 1407 \\ 22 & 1407 \\ 22 & 1408 \\ 22 & 1409 \\ 22 & 1409 \\ 22 & 1410\end{array}$

october

\begin{tabular}{|c|c|c|c|c|}
\hline $\begin{array}{l}03 \\
03 \\
03 \\
03 \\
03 \\
03 \\
03 \\
03\end{array}$ & $\begin{array}{l}1417 \\
1417 \\
1418 \\
1419 \\
1419 \\
1420 \\
1420 \\
1421\end{array}$ & $\begin{array}{l}0.2 \\
3.1 \\
4.8 \\
10 \\
15 \\
20 \\
24 \\
30\end{array}$ & $\begin{array}{l}500 \\
500 \\
500 \\
500 \\
500 \\
500 \\
500 \\
500\end{array}$ & $\begin{array}{l}485 \\
483 \\
482 \\
483 \\
483 \\
481 \\
482 \\
481\end{array}$ \\
\hline $\begin{array}{l}03 \\
03 \\
03 \\
03 \\
03 \\
03 \\
03 \\
03 \\
03\end{array}$ & $\begin{array}{l}1409 \\
1409 \\
1410 \\
1410 \\
1411 \\
1411 \\
1412 \\
1412 \\
1413\end{array}$ & $\begin{array}{c}0.2 \\
2.9 \\
5.0 \\
10 \\
15 \\
20 \\
25 \\
30 \\
32\end{array}$ & $\begin{array}{lll}9 & 0 & 0 \\
9 & 0 & 0 \\
9 & 0 & 0 \\
9 & 0 & 0 \\
9 & 0 & 0 \\
9 & 0 & 0 \\
9 & 0 & 0 \\
9 & 0 & 0 \\
9 & 0 & 0\end{array}$ & $\begin{array}{l}484 \\
484 \\
483 \\
486 \\
486 \\
488 \\
488 \\
476 \\
475\end{array}$ \\
\hline $\begin{array}{l}03 \\
03 \\
03 \\
03 \\
03 \\
03 \\
03 \\
03\end{array}$ & $\begin{array}{l}1354 \\
1354 \\
1355 \\
1356 \\
1356 \\
1357 \\
1358 \\
1359\end{array}$ & $\begin{array}{l}0.4 \\
2.8 \\
4.8 \\
9.9 \\
15 \\
20 \\
25 \\
28\end{array}$ & $\begin{array}{l}1,400 \\
1,400 \\
1,400 \\
1,400 \\
1,400 \\
1,400 \\
1,400 \\
1,400\end{array}$ & $\begin{array}{l}484 \\
484 \\
484 \\
482 \\
479 \\
482 \\
481 \\
480\end{array}$ \\
\hline $\begin{array}{l}17 \\
17 \\
17 \\
17 \\
17 \\
17 \\
17 \\
17 \\
17\end{array}$ & $\begin{array}{l}1418 \\
1418 \\
1419 \\
1419 \\
1420 \\
1421 \\
1421 \\
1422 \\
1422\end{array}$ & $\begin{array}{c}0.9 \\
3.2 \\
5.1 \\
9.9 \\
15 \\
20 \\
25 \\
30 \\
31\end{array}$ & $\begin{array}{l}500 \\
500 \\
500 \\
500 \\
500 \\
500 \\
500 \\
500 \\
500\end{array}$ & $\begin{array}{l}508 \\
507 \\
507 \\
506 \\
499 \\
487 \\
489 \\
495 \\
487\end{array}$ \\
\hline
\end{tabular}

$\begin{array}{rr}1.0 & 1,400 \\ 2.8 & 1,400 \\ 4.9 & 1,400 \\ 10 & 1,400 \\ 15 & 1,400 \\ 20 & 1,400 \\ 25 & 1,400 \\ 27 & 1,400\end{array}$

492
491
489
489
489
490
490

7.4
7.4
7.4
7.4
7.4
7.4
7.4
7.4

26.1
25.8
25.3
24.0
23.7
23.2
22.9
22.8

7.5

7.5

7.5

7.5

7.5

7.5

7.5

7.5

7.5

7.5

7.5

7.5

7.5

7.5

7.5

7.5

7.5

26.7

26.6

26.2

23.7

22.3

21.8

21.1

\section{7}

25.7
25.7

24.1

22.2

21.6

21.0

21.0

7.5

7.5

7.5

7.5

7.5

7.5

$7 \cdot 5$
$7 \cdot 5$

21.0

25.9

25.2

24.3

23.4

22.2

21.5

21.4

21.2

7.4

7.4

7.4

7.4

7.4

7.4
7.4

7.4
7.4

20.6

20.6

20.3

19.4

18.5

18.0

17.8

17.7

17.7

$\begin{array}{lll}\ldots & 7.1 & 89 \\ \ldots & 7.1 & 89 \\ -. & 7.1 & 88 \\ \ldots & 7.1 & 86 \\ \ldots & 7.1 & 86 \\ -- & 7.1 & 85 \\ -. & 7.0 & 83 \\ -- & 7.0 & 82\end{array}$

- 8.2

105

-. 8.2

- 8.2

.
.

- 8.2

. 8.1

-
$\ldots \quad 8$

105

104

99

97

96

94

92

.. 8.2
- 8.3

-
$\ldots$

. 8.3

. 8.2

$\begin{array}{ll}\ldots & 8.2 \\ \ldots & 8.3\end{array}$

- 8.2

$\begin{array}{ll}- & 8.2 \\ - & 8.1\end{array}$

- 8.1

-
- 8.2

- $\quad 8.2 \quad 103$

$\begin{array}{lll}- & 8.2 & 102\end{array}$

$\begin{array}{lll}\text { - } & 8.3 & 102\end{array}$

- 8.3

. 8.2

-
$\ldots$

- 8.0

-. 8.3

.-
$\ldots \quad 8$

- 8.3

- 8.4

\begin{tabular}{ll}
- & 8.4 \\
- & 8.5 \\
\hline & 8.4
\end{tabular}

-

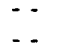

$\cdots$

8.4

8.3

8.3

- 8.2

104

105
104

100

97

96
93

94

94 
Table 2. Water-quality data for station 403156080373201, Ohio River at river mile 54.0, June to October, 1995, Continued.

[ft $=$ feet $; \mu \mathrm{S} / \mathrm{cm}=$ microsiemens per centimeter $;{ }^{\circ} \mathrm{C}=$ degrees Celsius; $\mathrm{mg} / \mathrm{L}=\mathrm{milligrams}$ per liter; $\cdots$ = data not collected]

\begin{tabular}{|c|c|c|c|c|c|c|c|c|c|}
\hline Date & Time & $\begin{array}{l}\text { Sampling } \\
\text { depth } \\
\text { (ft) }\end{array}$ & $\begin{array}{c}\text { Sample } \\
\text { location } \\
\text { (ft from } \\
\text { left bank) }\end{array}$ & $\begin{array}{l}\text { Specific } \\
\text { conduct- } \\
\text { ance } \\
(\mu \mathrm{s} / \mathrm{cm})\end{array}$ & $\begin{array}{l}\mathrm{pH} \\
\text { (stan- } \\
\text { dard } \\
\text { units) }\end{array}$ & $\begin{array}{l}\text { Temper- } \\
\text { ature, } \\
\text { water } \\
\left({ }^{\circ} \mathrm{C}\right)\end{array}$ & $\begin{array}{l}\text { Trans- } \\
\text { parency } \\
\text { (Secchi } \\
\text { disk) } \\
\text { (ft) }\end{array}$ & $\begin{array}{l}\text { Dissolved } \\
\text { oxygen } \\
\text { (mg/L) }\end{array}$ & $\begin{array}{c}\text { Dissolved } \\
\text { oxygen } \\
\text { (percent } \\
\text { satura- } \\
\text { tion) }\end{array}$ \\
\hline \multicolumn{10}{|c|}{ October } \\
\hline 17 & 1405 & 0.9 & 900 & 502 & 7.4 & 20.4 & $\cdots$ & 8.5 & 95 \\
\hline 17 & 1405 & 3.2 & 900 & 502 & 7.4 & 20.1 & - & 8.3 & 92 \\
\hline 17 & 1406 & 5.2 & 900 & 504 & 7.4 & 19.5 & 3.5 & 8.2 & 90 \\
\hline 17 & 1407 & 10 & 900 & 503 & 7.4 & 18.9 & - - & 8.3 & 90 \\
\hline 17 & 1407 & 15 & 900 & 499 & 7.4 & 18.4 & $\cdots$ & 8.5 & 92 \\
\hline 17 & 1408 & 20 & 900 & 491 & 7.4 & 18.1 & $\cdots$ & 8.4 & 90 \\
\hline 17 & 1408 & 25 & 900 & 493 & 7.4 & 17.9 & $-\cdot$ & 8.4 & 89 \\
\hline 17 & 1409 & 30 & 900 & 499 & 7.4 & 17.8 & - & 8.4 & 89 \\
\hline 17 & 1409 & 35 & 900 & 490 & 7.4 & 17.8 & - & 8.4 & 89 \\
\hline 17 & 1410 & 38 & 900 & 490 & 7.4 & 17.8 & -- & 8.3 & 88 \\
\hline 17 & 1359 & 1.0 & 1,400 & 502 & 7.4 & 21.0 & $\cdots$ & 8.8 & 99 \\
\hline 17 & 1359 & 3.2 & 1,400 & 503 & 7.4 & 20.4 & - & 8.5 & 95 \\
\hline 17 & 1400 & 5.2 & 1,400 & 501 & 7.4 & 19.7 & - & 8.6 & 95 \\
\hline 17 & 1400 & 10 & 1,400 & 501 & 7.4 & 18.9 & - & 8.5 & 92 \\
\hline 17 & 1401 & 15 & 1,400 & 499 & 7.4 & 18.5 & - & 8.4 & 90 \\
\hline 17 & 1401 & 20 & 1,400 & 496 & 7.4 & 18.1 & - & 8.3 & 88 \\
\hline 17 & 1402 & 25 & 1,400 & 490 & 7.4 & 17.9 & $\cdots$ & 8.4 & 89 \\
\hline 17 & 1402 & 27 & 1,400 & 490 & 7.4 & 17.9 & - & 8.2 & 87 \\
\hline
\end{tabular}


Table 3. Water-quality data for station 403139080373801, Ohio River at river mile 54.4, June to October, 1995.

[ft $=$ feet $; \mu \mathrm{S} / \mathrm{cm}=$ microsiemens per centimeter $;{ }^{\circ} \mathrm{C}=$ degrees Celsius; $\mathrm{mg} / \mathrm{L}=\mathrm{mili}$ igrams per liter: - = data not collected]

\begin{tabular}{|c|c|c|c|c|c|c|c|c|c|}
\hline Date & Time & $\begin{array}{l}\text { Sampling } \\
\text { depth } \\
\text { (ft) }\end{array}$ & $\begin{array}{c}\text { Sample } \\
\text { location } \\
\text { (Et from } \\
\text { left bank) }\end{array}$ & $\begin{array}{l}\text { Specific } \\
\text { conduct- } \\
\text { ance } \\
(\mu \mathrm{S} / \mathrm{cm})\end{array}$ & $\begin{array}{l}\text { pH } \\
\text { (stan- } \\
\text { dard } \\
\text { units) }\end{array}$ & $\begin{array}{c}\text { Temper- } \\
\text { ature, } \\
\text { water } \\
\left({ }^{\circ} \mathrm{C}\right)\end{array}$ & $\begin{array}{l}\text { Trans- } \\
\text { parency } \\
\text { (secchi } \\
\text { disk) } \\
\text { (ft) }\end{array}$ & $\begin{array}{l}\text { Dissolved } \\
\text { oxygen } \\
(\mathrm{mg} / \mathrm{L})\end{array}$ & $\begin{array}{c}\text { Dissolve } \\
\text { oxygen } \\
\text { (percen } \\
\text { satura } \\
\text { tion) }\end{array}$ \\
\hline June & & & & & & & & & \\
\hline $\begin{array}{l}27 \\
27 \\
27 \\
27 \\
27 \\
27 \\
27 \\
27\end{array}$ & $\begin{array}{l}1411 \\
1411 \\
1410 \\
1410 \\
1409 \\
1409 \\
1408 \\
1408\end{array}$ & $\begin{array}{l}0.5 \\
3.2 \\
5.3 \\
9.8 \\
15 \\
20 \\
25 \\
31\end{array}$ & $\begin{array}{l}1,100 \\
1,100 \\
1,100 \\
1,100 \\
1,100 \\
1,100 \\
1,100 \\
1,100\end{array}$ & $\begin{array}{l}380 \\
381 \\
380 \\
380 \\
378 \\
380 \\
380 \\
380\end{array}$ & $\begin{array}{l}7.5 \\
7.5 \\
7.5 \\
7.5 \\
7.5 \\
7.5 \\
7.5 \\
7.5\end{array}$ & $\begin{array}{l}28.7 \\
28.8 \\
28.8 \\
28.3 \\
27.3 \\
25.4 \\
25.4 \\
25.4\end{array}$ & $\begin{array}{l}-- \\
-- \\
-- \\
-- \\
-- \\
-- \\
-\end{array}$ & $\begin{array}{l}6.9 \\
6.9 \\
6.9 \\
6.9 \\
6.9 \\
6.9 \\
6.9 \\
6.9\end{array}$ & $\begin{array}{l}92 \\
91 \\
91 \\
91 \\
89 \\
87 \\
86 \\
85\end{array}$ \\
\hline $\begin{array}{r}\text { Ju1y } \\
11 \\
11 \\
11 \\
11 \\
11 \\
11 \\
11 \\
11 \\
11\end{array}$ & $\begin{array}{l}1118 \\
1118 \\
1119 \\
11119 \\
1120 \\
1120 \\
1121 \\
1121 \\
1122\end{array}$ & $\begin{array}{l}0.3 \\
3.0 \\
5.1 \\
9.6 \\
15 \\
20 \\
25 \\
30 \\
31\end{array}$ & $\begin{array}{l}1,100 \\
1,100 \\
1,100 \\
1,100 \\
1,1000 \\
1,100 \\
1,100 \\
1,100 \\
1,100\end{array}$ & $\begin{array}{l}428 \\
429 \\
426 \\
427 \\
432 \\
432 \\
422 \\
429 \\
427\end{array}$ & $\begin{array}{l}7.3 \\
7.3 \\
7.3 \\
7.3 \\
7.3 \\
7.3 \\
7.3 \\
7.3 \\
7.3\end{array}$ & $\begin{array}{l}29.6 \\
29.8 \\
29.7 \\
29.4 \\
29.2 \\
27.0 \\
26.2 \\
26.1 \\
26.1\end{array}$ & $\begin{array}{l}- \\
\cdots \\
- \\
\cdots \\
-- \\
-- \\
-- \\
-\end{array}$ & $\begin{array}{l}7.4 \\
7.4 \\
7.3 \\
7.3 \\
7.3 \\
7.2 \\
7.2 \\
7.1 \\
7.1\end{array}$ & $\begin{array}{l}99 \\
99 \\
98 \\
98 \\
97 \\
92 \\
91 \\
90 \\
89\end{array}$ \\
\hline $\begin{array}{l}25 \\
25 \\
25 \\
25 \\
25 \\
25 \\
25 \\
25\end{array}$ & $\begin{array}{l}1203 \\
1203 \\
1204 \\
1204 \\
1205 \\
1205 \\
1206 \\
1206\end{array}$ & $\begin{array}{l}0.5 \\
3.0 \\
5.0 \\
10 \\
15 \\
20 \\
25 \\
30\end{array}$ & $\begin{array}{l}1,100 \\
1,100 \\
1,100 \\
1,100 \\
1,100 \\
1,100 \\
1,100 \\
1,100\end{array}$ & $\begin{array}{l}429 \\
426 \\
426 \\
427 \\
423 \\
428 \\
428 \\
431\end{array}$ & $\begin{array}{l}7.4 \\
7.4 \\
7.4 \\
7.4 \\
7.4 \\
7.4 \\
7.4 \\
7.4\end{array}$ & $\begin{array}{l}33.1 \\
32.9 \\
33.1 \\
32.4 \\
31.3 \\
30.0 \\
27.8 \\
27.5\end{array}$ & $\begin{array}{l}-- \\
- \\
-- \\
-- \\
-- \\
-\end{array}$ & $\begin{array}{l}6.9 \\
6.8 \\
6.7 \\
6.7 \\
6.7 \\
6.7 \\
6.5 \\
6.5\end{array}$ & $\begin{array}{l}98 \\
97 \\
96 \\
94 \\
93 \\
90 \\
85 \\
84\end{array}$ \\
\hline $\begin{array}{c}\text { Augus } \\
08 \\
08 \\
08 \\
08 \\
08 \\
08 \\
08 \\
08\end{array}$ & $\begin{array}{l}\text { t } \\
1031 \\
1032 \\
1033 \\
1033 \\
1034 \\
1035 \\
1036 \\
1037\end{array}$ & $\begin{array}{l}0.9 \\
3.0 \\
4.9 \\
10 \\
15 \\
20 \\
25 \\
31\end{array}$ & $\begin{array}{l}1,100 \\
1,100 \\
1,100 \\
1,100 \\
1,100 \\
1,100 \\
1,100 \\
1,100\end{array}$ & $\begin{array}{l}451 \\
450 \\
452 \\
453 \\
448 \\
447 \\
449 \\
452\end{array}$ & $\begin{array}{l}7.5 \\
7.5 \\
7.5 \\
7.5 \\
7.5 \\
7.5 \\
7.5 \\
7.5\end{array}$ & $\begin{array}{l}32.1 \\
31.9 \\
31.6 \\
31.3 \\
31.2 \\
29.2 \\
28.6 \\
28.6\end{array}$ & $\begin{array}{l}-- \\
-- \\
-- \\
-- \\
-- \\
-- \\
-\end{array}$ & $\begin{array}{l}6.9 \\
7.0 \\
6.9 \\
6.9 \\
6.9 \\
6.8 \\
6.8 \\
6.8\end{array}$ & $\begin{array}{l}97 \\
97 \\
95 \\
94 \\
94 \\
90 \\
90 \\
89\end{array}$ \\
\hline $\begin{array}{l}22 \\
22 \\
22 \\
22 \\
22 \\
22 \\
22 \\
22 \\
22\end{array}$ & $\begin{array}{l}1040 \\
1040 \\
1041 \\
1041 \\
1042 \\
1042 \\
1043 \\
1043 \\
1044\end{array}$ & $\begin{array}{l}0.4 \\
2.6 \\
5.0 \\
9.8 \\
15 \\
20 \\
25 \\
30 \\
31\end{array}$ & $\begin{array}{l}1,100 \\
1,100 \\
1,100 \\
1,100 \\
1,100 \\
1,100 \\
1,100 \\
1,100 \\
1,100\end{array}$ & $\begin{array}{l}480 \\
480 \\
480 \\
481 \\
481 \\
484 \\
481 \\
477 \\
479\end{array}$ & $\begin{array}{l}7.6 \\
7.6 \\
7.6 \\
7.6 \\
7.6 \\
7.6 \\
7.5 \\
7.5 \\
7.5\end{array}$ & $\begin{array}{l}36.6 \\
36.6 \\
36.2 \\
35.7 \\
35.3 \\
32.5 \\
30.2 \\
30.0 \\
29.9\end{array}$ & $\begin{array}{l}-- \\
- \\
- \\
\cdots \\
- \\
- \\
- \\
-\end{array}$ & $\begin{array}{l}6.8 \\
7.0 \\
7.0 \\
7.0 \\
6.9 \\
6.8 \\
7.0 \\
6.8 \\
6.7\end{array}$ & $\begin{array}{r}102 \\
105 \\
104 \\
103 \\
101 \\
95 \\
94 \\
91 \\
90\end{array}$ \\
\hline
\end{tabular}


Table 3. Water-quality data for station 403139080373801 , Ohio River at river mile 54.4, June to October, 1995, Continued.

[ft $=$ feet $; \mu \mathrm{s} / \mathrm{cm}=$ microsiemens per centimeter $;{ }^{\circ} \mathrm{C}=$ degrees Celsius $\mathrm{mg} / \mathrm{L}=$ milligrams per liter; .. = data not collected]

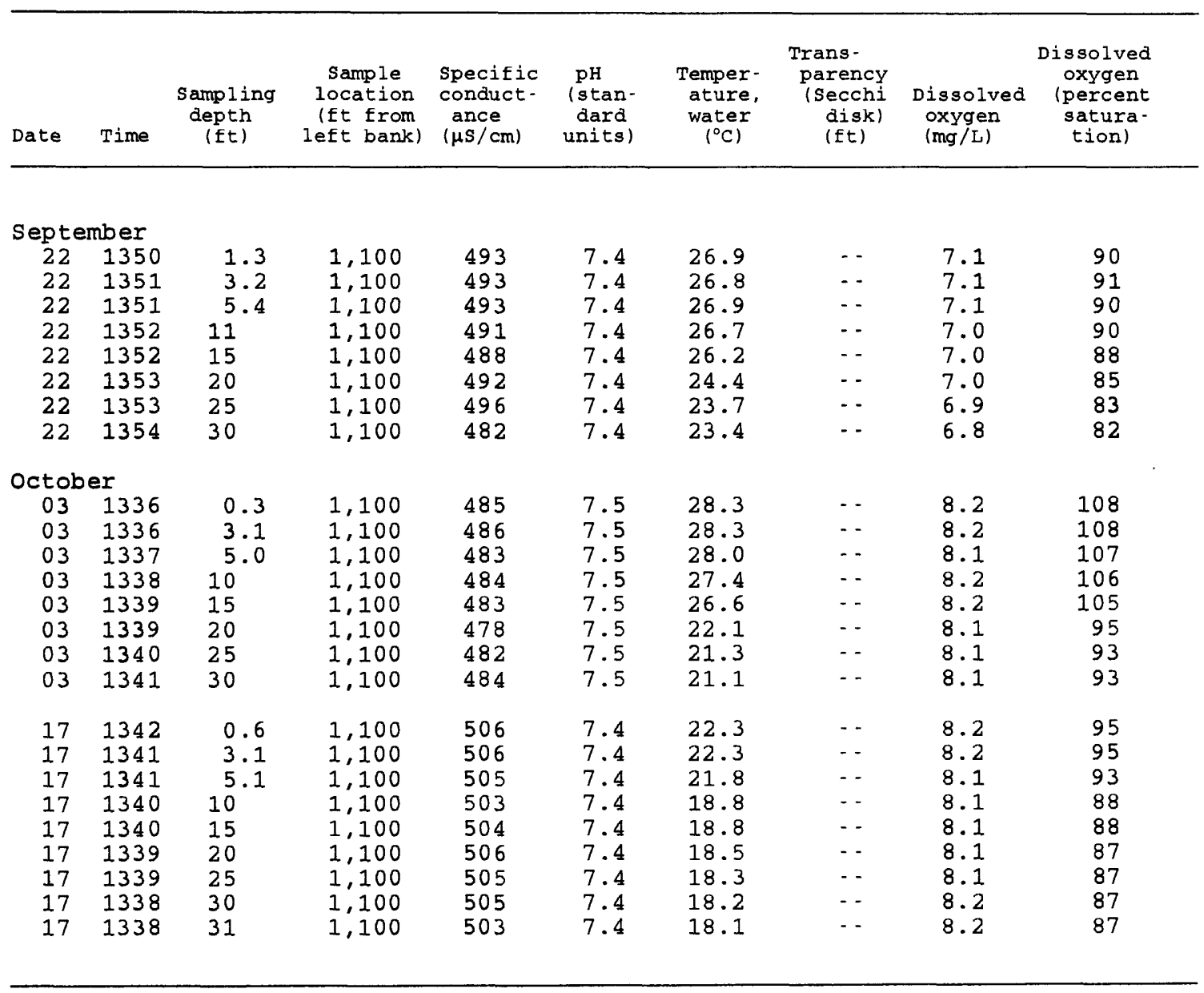


Table 4. Water-quality data for station 403115080371801 , Ohio River at river mile 54.8, June to October, 1995.

[ft $=$ feet $; \mu \mathrm{s} / \mathrm{cm}=$ microsiemens per centimeter $;{ }^{\circ} \mathrm{C}=$ degrees Celsius;

$\mathrm{mg} / \mathrm{L}=\mathrm{milligrams}$ per liter; $\cdots=$ data not collected]

\begin{tabular}{|c|c|c|c|c|c|c|c|c|c|}
\hline Date & Time & $\begin{array}{l}\text { Sampling } \\
\text { depth } \\
\text { (ft) }\end{array}$ & $\begin{array}{c}\text { Sample } \\
\text { location } \\
\text { (Et from } \\
\text { left bank) }\end{array}$ & $\begin{array}{l}\text { Specific } \\
\text { conduct - } \\
\text { ance } \\
(\mu \mathrm{s} / \mathrm{cm})\end{array}$ & $\begin{array}{l}\text { pH } \\
\text { (stan- } \\
\text { dard } \\
\text { units) }\end{array}$ & $\begin{array}{c}\text { Temper- } \\
\text { ature, } \\
\text { water } \\
\left({ }^{\circ} \mathrm{C}\right)\end{array}$ & $\begin{array}{c}\text { Trans - } \\
\text { parency } \\
\text { (Secchi } \\
\text { disk) } \\
\text { (Et) }\end{array}$ & $\begin{array}{c}\text { Dissolved } \\
\text { oxygen } \\
\text { (mg/L) }\end{array}$ & $\begin{array}{c}\text { Dissolved } \\
\text { oxygen } \\
\text { (percent } \\
\text { satura- } \\
\text { tion) }\end{array}$ \\
\hline
\end{tabular}

July

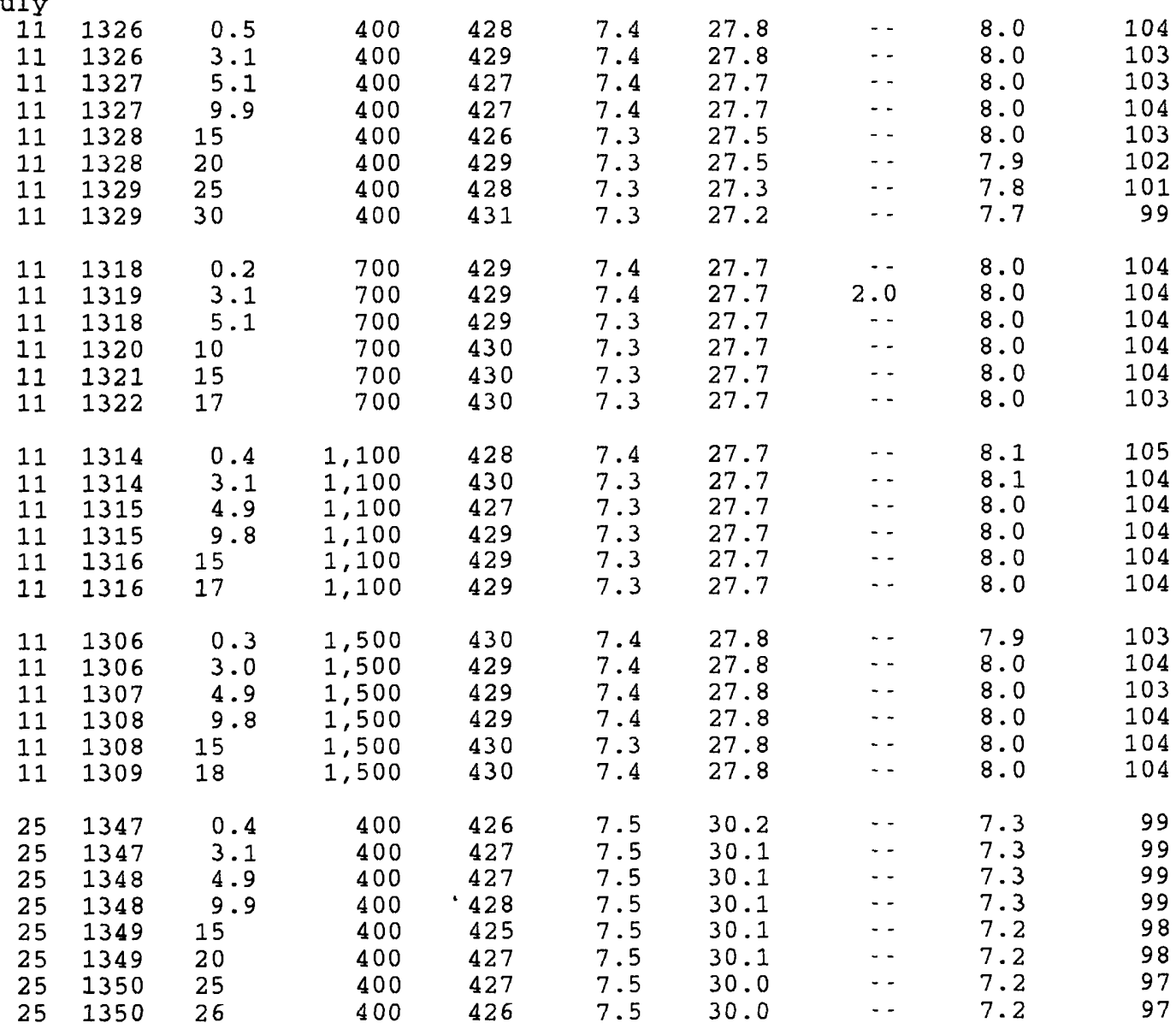


Table 4. Water-quality data for station 403115080371801, Ohio River at river mile 54.8, June to October, 1995, Continued.

[ft $=$ feet; $\mu \mathrm{s} / \mathrm{cm}=$ microsiemens per centimeter; ${ }^{\circ} \mathrm{C}=$ degrees Celsius; $\mathrm{mg} / \mathrm{L}=\mathrm{milligrams}$ per liter;.. = data not collected]

\begin{tabular}{|c|c|c|c|c|c|c|c|c|c|}
\hline Date & Time & $\begin{array}{l}\text { Sampling } \\
\text { depth } \\
\text { (ft) }\end{array}$ & $\begin{array}{c}\text { Sample } \\
\text { location } \\
\text { (ft from } \\
\text { left bank) }\end{array}$ & $\begin{array}{l}\text { Specific } \\
\text { conduct- } \\
\text { ance } \\
(\mu \mathrm{S} / \mathrm{cm})\end{array}$ & $\begin{array}{l}\mathrm{pH} \\
\text { (stan- } \\
\text { dard } \\
\text { units) }\end{array}$ & $\begin{array}{c}\text { Temper- } \\
\text { ature, } \\
\text { water } \\
\left({ }^{\circ} \mathrm{C}\right)\end{array}$ & $\begin{array}{l}\text { Trans- } \\
\text { parency } \\
\text { (secchi } \\
\text { disk) } \\
\text { (ft) }\end{array}$ & $\begin{array}{l}\text { Dissolved } \\
\text { oxygen } \\
(\mathrm{mg} / \mathrm{L})\end{array}$ & $\begin{array}{c}\text { Disgolved } \\
\text { oxygen } \\
\text { (percent } \\
\text { gatura- } \\
\text { tion) }\end{array}$ \\
\hline
\end{tabular}

\begin{tabular}{|c|c|c|c|c|c|c|c|c|c|}
\hline $\begin{array}{r}\text { July } \\
25 \\
25 \\
25 \\
25 \\
25\end{array}$ & $\begin{array}{l}1343 \\
1343 \\
1344 \\
1344 \\
1345\end{array}$ & $\begin{array}{l}0.7 \\
3.2 \\
5.1 \\
10 \\
15\end{array}$ & $\begin{array}{l}700 \\
700 \\
700 \\
700 \\
700\end{array}$ & $\begin{array}{l}425 \\
427 \\
427 \\
425 \\
429\end{array}$ & $\begin{array}{l}7.5 \\
7.5 \\
7.5 \\
7.5 \\
7.5\end{array}$ & $\begin{array}{l}30.1 \\
30.0 \\
30.0 \\
30.0 \\
29.9\end{array}$ & $\begin{array}{r}- \\
1.5 \\
-- \\
- \\
-\end{array}$ & $\begin{array}{l}7.4 \\
7.4 \\
7.4 \\
7.4 \\
7.4\end{array}$ & $\begin{array}{l}100 \\
100 \\
100 \\
100 \\
100\end{array}$ \\
\hline $\begin{array}{l}25 \\
25 \\
25 \\
25 \\
25 \\
25\end{array}$ & $\begin{array}{l}1339 \\
1339 \\
1340 \\
1340 \\
1341 \\
1341\end{array}$ & $\begin{array}{r}0.2 \\
3.1 \\
5.0 \\
9.8 \\
15 \\
19\end{array}$ & $\begin{array}{l}1,100 \\
1,100 \\
1,100 \\
1,100 \\
1,100 \\
1,100\end{array}$ & $\begin{array}{l}427 \\
425 \\
426 \\
425 \\
425 \\
428\end{array}$ & $\begin{array}{l}7.5 \\
7.5 \\
7.5 \\
7.5 \\
7.5 \\
7.5\end{array}$ & $\begin{array}{l}29.8 \\
29.8 \\
29.8 \\
29.8 \\
29.8 \\
29.8\end{array}$ & $\begin{array}{l}- \\
-- \\
-- \\
-- \\
--\end{array}$ & $\begin{array}{l}7.4 \\
7.4 \\
7.4 \\
7.4 \\
7.4 \\
7.4\end{array}$ & $\begin{array}{l}100 \\
100 \\
100 \\
100 \\
100 \\
100\end{array}$ \\
\hline $\begin{array}{l}25 \\
25 \\
25 \\
25 \\
25\end{array}$ & $\begin{array}{l}1334 \\
1334 \\
1335 \\
1335 \\
1336\end{array}$ & $\begin{array}{r}0.4 \\
3.0 \\
5.1 \\
10 \\
13\end{array}$ & $\begin{array}{l}1,500 \\
1,500 \\
1,500 \\
1,500 \\
1,500\end{array}$ & $\begin{array}{l}426 \\
426 \\
426 \\
427 \\
427\end{array}$ & $\begin{array}{l}7.5 \\
7.5 \\
7.5 \\
7.5 \\
7.5\end{array}$ & $\begin{array}{l}29.8 \\
29.8 \\
29.8 \\
29.7 \\
29.7\end{array}$ & $\begin{array}{l}-- \\
-- \\
-- \\
--\end{array}$ & $\begin{array}{l}7.3 \\
7.4 \\
7.4 \\
7.4 \\
7.4\end{array}$ & $\begin{array}{r}99 \\
99 \\
99 \\
100 \\
100\end{array}$ \\
\hline $\begin{array}{c}\text { Augus } \\
08 \\
08 \\
08 \\
08 \\
08 \\
08 \\
08 \\
08\end{array}$ & $\begin{array}{l}0832 \\
0832 \\
0833 \\
0833 \\
0834 \\
0834 \\
0835 \\
0835\end{array}$ & $\begin{array}{c}0.4 \\
3.0 \\
5.1 \\
9.1 \\
14 \\
21 \\
26 \\
30\end{array}$ & $\begin{array}{l}400 \\
400 \\
400 \\
400 \\
400 \\
400 \\
400 \\
400\end{array}$ & $\begin{array}{l}452 \\
451 \\
453 \\
451 \\
455 \\
454 \\
447 \\
452\end{array}$ & $\begin{array}{l}7.5 \\
7.5 \\
7.5 \\
7.5 \\
7.5 \\
7.5 \\
7.5 \\
7.5\end{array}$ & $\begin{array}{l}28.9 \\
28.9 \\
28.9 \\
28.9 \\
29.0 \\
29.0 \\
29.0 \\
29.0\end{array}$ & $\begin{array}{l}-- \\
-- \\
-- \\
-- \\
-- \\
-- \\
--\end{array}$ & $\begin{array}{l}7.9 \\
7.9 \\
7.9 \\
7.9 \\
7.9 \\
7.9 \\
7.8 \\
7.8\end{array}$ & $\begin{array}{l}104 \\
104 \\
104 \\
104 \\
104 \\
104 \\
103 \\
103\end{array}$ \\
\hline $\begin{array}{l}08 \\
08 \\
08 \\
08 \\
08 \\
08\end{array}$ & $\begin{array}{l}0838 \\
0838 \\
0839 \\
0839 \\
0840 \\
0840\end{array}$ & $\begin{array}{r}0.8 \\
3.2 \\
5.1 \\
10 \\
15 \\
19\end{array}$ & $\begin{array}{l}700 \\
700 \\
700 \\
700 \\
700 \\
700\end{array}$ & $\begin{array}{l}452 \\
455 \\
454 \\
450 \\
452 \\
451\end{array}$ & $\begin{array}{l}7.5 \\
7.5 \\
7.5 \\
7.5 \\
7.5 \\
7.5\end{array}$ & $\begin{array}{l}29.2 \\
29.2 \\
29.2 \\
29.1 \\
29.1 \\
29.0\end{array}$ & $\begin{array}{l}-- \\
-- \\
-- \\
-- \\
-- \\
--\end{array}$ & $\begin{array}{l}7.7 \\
7.8 \\
7.8 \\
7.8 \\
7.7 \\
7.8\end{array}$ & $\begin{array}{l}102 \\
104 \\
104 \\
103 \\
102 \\
103\end{array}$ \\
\hline $\begin{array}{l}08 \\
08 \\
08 \\
08 \\
08 \\
08\end{array}$ & $\begin{array}{l}0845 \\
0845 \\
0846 \\
0846 \\
0847 \\
0847\end{array}$ & $\begin{array}{r}0.4 \\
2.7 \\
5.6 \\
9.4 \\
13 \\
18\end{array}$ & $\begin{array}{l}1,100 \\
1,100 \\
1,100 \\
1,100 \\
1,100 \\
1,100\end{array}$ & $\begin{array}{l}450 \\
452 \\
454 \\
455 \\
449 \\
458\end{array}$ & $\begin{array}{l}7.5 \\
7.5 \\
7.5 \\
7.5 \\
7.5 \\
7.5\end{array}$ & $\begin{array}{l}29.4 \\
29.4 \\
29.4 \\
29.3 \\
29.3 \\
29.3\end{array}$ & $\begin{array}{l}-- \\
-- \\
-- \\
- \\
-\end{array}$ & $\begin{array}{l}7.7 \\
7.8 \\
7.8 \\
7.8 \\
7.8 \\
7.8\end{array}$ & $\begin{array}{l}103 \\
104 \\
104 \\
103 \\
103 \\
104\end{array}$ \\
\hline
\end{tabular}


Table 4. Water-quality data for station 403115080371801, Ohio River at river mile 54.8, June to October, 1995, Continued.

[ft $=$ feet $; \mu \mathrm{s} / \mathrm{cm}=$ microsiemens per centimeter; ${ }^{\circ} \mathrm{C}=$ degrees Celsius;

$\mathrm{mg} / L=$ milligrams per liter; $\cdots$ = data not collected]

\begin{tabular}{|c|c|c|c|c|c|c|c|c|c|}
\hline Date & Time & $\begin{array}{l}\text { Sampling } \\
\text { depth } \\
\text { (ft) }\end{array}$ & $\begin{array}{c}\text { Sample } \\
\text { location } \\
\text { (ft from } \\
\text { left bank) }\end{array}$ & $\begin{array}{l}\text { Specific } \\
\text { conduct- } \\
\text { ance } \\
(\mu \mathrm{s} / \mathrm{cm})\end{array}$ & $\begin{array}{l}\text { pH } \\
\text { (stan- } \\
\text { dard } \\
\text { units) }\end{array}$ & $\begin{array}{c}\text { Temper- } \\
\text { ature, } \\
\text { water } \\
\left({ }^{\circ} \mathrm{C}\right)\end{array}$ & $\begin{array}{l}\text { Trans- } \\
\text { parency } \\
\text { (Secchi } \\
\text { disk) } \\
\text { (ft) }\end{array}$ & $\begin{array}{l}\text { Dissolved } \\
\text { oxygen } \\
(\mathrm{mg} / \mathrm{L})\end{array}$ & $\begin{array}{c}\text { Dissolved } \\
\text { oxygen } \\
\text { (percent } \\
\text { satura- } \\
\text { tion) }\end{array}$ \\
\hline
\end{tabular}

\begin{tabular}{|c|c|c|c|c|c|c|c|c|c|}
\hline \multicolumn{10}{|c|}{ August } \\
\hline $\begin{array}{l}08 \\
08 \\
08 \\
08 \\
08\end{array}$ & $\begin{array}{l}0911 \\
0911 \\
0912 \\
0912 \\
0913\end{array}$ & $\begin{array}{r}0.2 \\
3.1 \\
5.2 \\
9.7 \\
14\end{array}$ & $\begin{array}{l}1,500 \\
1,500 \\
1,500 \\
1,500 \\
1,500\end{array}$ & $\begin{array}{l}451 \\
454 \\
452 \\
452 \\
451\end{array}$ & $\begin{array}{l}7.5 \\
7.5 \\
7.5 \\
7.5 \\
7.5\end{array}$ & $\begin{array}{l}29.5 \\
29.5 \\
29.5 \\
29.6 \\
29.6\end{array}$ & $\begin{array}{l}-- \\
-- \\
-- \\
--\end{array}$ & $\begin{array}{l}7.6 \\
7.6 \\
7.5 \\
7.5 \\
7.5\end{array}$ & $\begin{array}{l}101 \\
101 \\
100 \\
100 \\
100\end{array}$ \\
\hline $\begin{array}{l}22 \\
22 \\
22 \\
22 \\
22\end{array}$ & $\begin{array}{l}1228 \\
1228 \\
1229 \\
1229 \\
1230\end{array}$ & $\begin{array}{r}0.9 \\
3.2 \\
5.1 \\
9.7 \\
11\end{array}$ & $\begin{array}{l}400 \\
400 \\
400 \\
400 \\
400\end{array}$ & $\begin{array}{l}480 \\
480 \\
480 \\
482 \\
479\end{array}$ & $\begin{array}{l}7.6 \\
7.6 \\
7.6 \\
7.6 \\
7.6\end{array}$ & $\begin{array}{l}32.8 \\
32.8 \\
32.9 \\
32.8 \\
32.8\end{array}$ & $\begin{array}{l}-- \\
-- \\
-- \\
--\end{array}$ & $\begin{array}{l}7.6 \\
7.5 \\
7.6 \\
7.5 \\
7.5\end{array}$ & $\begin{array}{l}107 \\
105 \\
107 \\
106 \\
105\end{array}$ \\
\hline $\begin{array}{l}22 \\
22 \\
22 \\
22 \\
22 \\
22\end{array}$ & $\begin{array}{l}1221 \\
1222 \\
1222 \\
1223 \\
1223 \\
1224\end{array}$ & $\begin{array}{l}1.0 \\
3.1 \\
5.2 \\
10 \\
15 \\
18\end{array}$ & $\begin{array}{l}700 \\
700 \\
700 \\
700 \\
700 \\
700\end{array}$ & $\begin{array}{l}481 \\
482 \\
481 \\
479 \\
481 \\
483\end{array}$ & $\begin{array}{l}7.6 \\
7.6 \\
7.6 \\
7.6 \\
7.6 \\
7.6\end{array}$ & $\begin{array}{l}32.8 \\
32.8 \\
32.8 \\
32.8 \\
32.8 \\
32.8\end{array}$ & $\begin{array}{c}- \\
3.0 \\
\ldots \\
\cdots \\
- \\
-\end{array}$ & $\begin{array}{l}7.6 \\
7.6 \\
7.6 \\
7.5 \\
7.6 \\
7.5\end{array}$ & $\begin{array}{l}107 \\
107 \\
106 \\
106 \\
107 \\
105\end{array}$ \\
\hline $\begin{array}{l}22 \\
22 \\
22 \\
22 \\
22\end{array}$ & $\begin{array}{l}1213 \\
1213 \\
1214 \\
1215 \\
1215\end{array}$ & $\begin{array}{r}0.5 \\
3.1 \\
5.1 \\
9.8 \\
15\end{array}$ & $\begin{array}{l}1,100 \\
1,100 \\
1,100 \\
1,100 \\
1,100\end{array}$ & $\begin{array}{l}481 \\
482 \\
479 \\
481 \\
482\end{array}$ & $\begin{array}{l}7.6 \\
7.6 \\
7.6 \\
7.6 \\
7.6\end{array}$ & $\begin{array}{l}32.7 \\
32.8 \\
32.7 \\
32.7 \\
32.7\end{array}$ & $\begin{array}{l}\cdots \\
\cdots \\
\cdots \\
-\end{array}$ & $\begin{array}{l}7.5 \\
7.5 \\
7.6 \\
7.6 \\
7.6\end{array}$ & $\begin{array}{l}106 \\
106 \\
107 \\
106 \\
107\end{array}$ \\
\hline $\begin{array}{l}22 \\
22 \\
22 \\
22 \\
22\end{array}$ & $\begin{array}{l}1201 \\
1201 \\
1202 \\
1202 \\
1203\end{array}$ & $\begin{array}{r}0.8 \\
2.9 \\
5.1 \\
10 \\
15\end{array}$ & $\begin{array}{l}1,500 \\
1,500 \\
1,500 \\
1,500 \\
1,500\end{array}$ & $\begin{array}{l}482 \\
481 \\
480 \\
481 \\
481\end{array}$ & $\begin{array}{l}7.6 \\
7.6 \\
7.6 \\
7.6 \\
7.6\end{array}$ & $\begin{array}{l}32.5 \\
32.5 \\
32.5 \\
32.5 \\
32.5\end{array}$ & $\begin{array}{l}-- \\
-- \\
-- \\
- \\
-\end{array}$ & $\begin{array}{l}7.5 \\
7.4 \\
7.5 \\
7.4 \\
7.4\end{array}$ & $\begin{array}{l}104 \\
104 \\
105 \\
104 \\
103\end{array}$ \\
\hline $\begin{array}{c}\text { Septe } \\
07 \\
07 \\
07 \\
07 \\
07 \\
07 \\
07\end{array}$ & $\begin{array}{l}\text { noer } \\
1713 \\
1713 \\
1714 \\
1714 \\
1715 \\
1715 \\
1716\end{array}$ & $\begin{array}{c}0.2 \\
3.0 \\
5.0 \\
10 \\
15 \\
20 \\
25\end{array}$ & $\begin{array}{l}400 \\
400 \\
400 \\
400 \\
400 \\
400 \\
400\end{array}$ & $\begin{array}{l}483 \\
484 \\
484 \\
483 \\
483 \\
481 \\
481\end{array}$ & $\begin{array}{l}7.9 \\
7.9 \\
7.9 \\
7.9 \\
7.9 \\
7.7 \\
7.6\end{array}$ & $\begin{array}{l}29.8 \\
29.9 \\
29.9 \\
29.9 \\
29.8 \\
29.4 \\
29.0\end{array}$ & $\begin{array}{l}\cdots \\
\cdots \\
\cdots \\
\cdots \\
\cdots\end{array}$ & $\begin{array}{l}7.6 \\
7.6 \\
7.6 \\
7.5 \\
7.4 \\
6.8 \\
6.6\end{array}$ & $\begin{array}{r}102 \\
102 \\
102 \\
101 \\
100 \\
91 \\
88\end{array}$ \\
\hline
\end{tabular}


Table 4. Water-quality data for station 403115080371801, Ohio River at river mile 54.8, June to October, 1995, Continued.

[ft $=$ feet $; \mu \mathrm{s} / \mathrm{cm}=$ microsiemens per centimeter; ${ }^{\circ} \mathrm{C}=$ degrees Celsius: $\mathrm{mg} / \mathrm{L}=$ milligrams per liter; -. = data not collected]

\begin{tabular}{|c|c|c|c|c|c|c|c|c|c|}
\hline Date & Time & $\begin{array}{l}\text { Sampling } \\
\text { depth } \\
\text { (ft) }\end{array}$ & $\begin{array}{c}\text { Sample } \\
\text { location } \\
\text { (ft from } \\
\text { left bank) }\end{array}$ & $\begin{array}{l}\text { Specific } \\
\text { conduct- } \\
\text { ance } \\
(\mu \mathrm{S} / \mathrm{cm})\end{array}$ & $\begin{array}{l}\text { pH } \\
\text { (stan- } \\
\text { dard } \\
\text { units) }\end{array}$ & $\begin{array}{c}\text { Temper- } \\
\text { ature, } \\
\text { water } \\
\left({ }^{\circ} \mathrm{C}\right)\end{array}$ & $\begin{array}{l}\text { Trans- } \\
\text { parency } \\
\text { (Secchi } \\
\text { disk) } \\
\text { (ft) }\end{array}$ & $\begin{array}{l}\text { Dissolved } \\
\text { oxygen } \\
(\mathrm{mg} / \mathrm{L})\end{array}$ & $\begin{array}{c}\text { Dissolved } \\
\text { oxygen } \\
\text { (percent } \\
\text { satura- } \\
\text { tion) }\end{array}$ \\
\hline
\end{tabular}

\begin{tabular}{|c|c|c|c|c|c|c|c|c|c|}
\hline $\begin{array}{l}\text { ept } \\
07 \\
07 \\
07 \\
07 \\
07 \\
07\end{array}$ & $\begin{array}{l}\text { nber } \\
1709 \\
1709 \\
1710 \\
1710 \\
1711 \\
1711\end{array}$ & $\begin{array}{l}0.2 \\
3.0 \\
5.0 \\
10 \\
15 \\
17\end{array}$ & $\begin{array}{l}700 \\
700 \\
700 \\
700 \\
700 \\
700\end{array}$ & $\begin{array}{l}484 \\
484 \\
484 \\
485 \\
485 \\
485\end{array}$ & $\begin{array}{l}7.9 \\
7.9 \\
7.9 \\
7.9 \\
7.9 \\
7.9\end{array}$ & $\begin{array}{l}29.8 \\
29.8 \\
29.8 \\
29.8 \\
29.8 \\
29.7\end{array}$ & $\begin{array}{l}- \\
- \\
- \\
- \\
-\end{array}$ & $\begin{array}{l}7.6 \\
7.6 \\
7.6 \\
7.6 \\
7.6 \\
7.5\end{array}$ & $\begin{array}{l}102 \\
102 \\
102 \\
102 \\
102 \\
101\end{array}$ \\
\hline $\begin{array}{l}07 \\
07 \\
07 \\
07 \\
07\end{array}$ & $\begin{array}{l}1705 \\
1705 \\
1706 \\
1706 \\
1707\end{array}$ & $\begin{array}{r}0.2 \\
3.0 \\
5.0 \\
10 \\
13\end{array}$ & $\begin{array}{l}1,100 \\
1,100 \\
1,100 \\
1,100 \\
1,100\end{array}$ & $\begin{array}{l}484 \\
484 \\
484 \\
484 \\
484\end{array}$ & $\begin{array}{l}7.9 \\
7.9 \\
7.9 \\
7.9 \\
7.9\end{array}$ & $\begin{array}{l}29.6 \\
29.6 \\
29.6 \\
29.6 \\
29.7\end{array}$ & $\begin{array}{l}-- \\
\cdots \\
\cdots \\
-\end{array}$ & $\begin{array}{l}7.7 \\
7.5 \\
7.6 \\
7.6 \\
7.6\end{array}$ & $\begin{array}{l}103 \\
102 \\
102 \\
103 \\
102\end{array}$ \\
\hline $\begin{array}{l}07 \\
07 \\
07 \\
07\end{array}$ & $\begin{array}{l}1700 \\
1700 \\
1701 \\
1702\end{array}$ & $\begin{array}{r}0.2 \\
3.0 \\
5.0 \\
10\end{array}$ & $\begin{array}{l}1,500 \\
1,500 \\
1,500 \\
1,500\end{array}$ & $\begin{array}{l}484 \\
484 \\
483 \\
482\end{array}$ & $\begin{array}{l}7.9 \\
8.0 \\
7.9 \\
7.8\end{array}$ & $\begin{array}{l}29.5 \\
29.6 \\
29.6 \\
28.0\end{array}$ & $\begin{array}{l}\cdots \\
\cdots \\
\cdots\end{array}$ & $\begin{array}{l}7.6 \\
7.8 \\
7.4 \\
7.1\end{array}$ & $\begin{array}{r}102 \\
105 \\
100 \\
93\end{array}$ \\
\hline $\begin{array}{l}22 \\
22 \\
22 \\
22 \\
22 \\
22 \\
22 \\
22\end{array}$ & $\begin{array}{l}1520 \\
1521 \\
1521 \\
1522 \\
1522 \\
1523 \\
1523 \\
1524\end{array}$ & $\begin{array}{c}1.1 \\
3.0 \\
5.0 \\
9.7 \\
15 \\
20 \\
25 \\
29\end{array}$ & $\begin{array}{l}400 \\
400 \\
400 \\
400 \\
400 \\
400 \\
400 \\
400\end{array}$ & $\begin{array}{l}489 \\
492 \\
491 \\
491 \\
492 \\
490 \\
490 \\
494\end{array}$ & $\begin{array}{l}7.5 \\
7.4 \\
7.4 \\
7.4 \\
7.4 \\
7.4 \\
7.4 \\
7.4\end{array}$ & $\begin{array}{l}24.8 \\
24.7 \\
24.7 \\
24.7 \\
24.8 \\
24.7 \\
24.7 \\
24.7\end{array}$ & $\begin{array}{l}-- \\
-- \\
-- \\
- \\
- \\
- \\
-\end{array}$ & $\begin{array}{l}7.8 \\
7.7 \\
7.5 \\
7.5 \\
7.6 \\
7.5 \\
7.6 \\
7.6\end{array}$ & $\begin{array}{l}96 \\
94 \\
91 \\
92 \\
93 \\
92 \\
93 \\
93\end{array}$ \\
\hline $\begin{array}{l}22 \\
22 \\
22 \\
22 \\
22 \\
22\end{array}$ & $\begin{array}{l}1527 \\
1527 \\
1528 \\
1528 \\
1529 \\
1529\end{array}$ & $\begin{array}{l}1.5 \\
2.9 \\
5.0 \\
10 \\
15 \\
17\end{array}$ & $\begin{array}{l}700 \\
700 \\
700 \\
700 \\
700 \\
700\end{array}$ & $\begin{array}{l}491 \\
489 \\
490 \\
494 \\
488 \\
490\end{array}$ & $\begin{array}{l}7.5 \\
7.5 \\
7.5 \\
7.4 \\
7.4 \\
7.4\end{array}$ & $\begin{array}{l}24.7 \\
24.8 \\
24.7 \\
24.7 \\
24.7 \\
24.7\end{array}$ & $\begin{array}{l}-- \\
\cdots \\
-- \\
-- \\
--\end{array}$ & $\begin{array}{l}7.6 \\
7.7 \\
7.7 \\
7.9 \\
7.8 \\
7.9\end{array}$ & $\begin{array}{l}94 \\
95 \\
94 \\
97 \\
96 \\
97\end{array}$ \\
\hline $\begin{array}{l}22 \\
22 \\
22 \\
22 \\
22 \\
22\end{array}$ & $\begin{array}{l}1531 \\
1532 \\
1533 \\
1533 \\
1534 \\
1535\end{array}$ & $\begin{array}{l}1.0 \\
3.0 \\
5.1 \\
8.5 \\
15 \\
20\end{array}$ & $\begin{array}{l}1,100 \\
1,100 \\
1,100 \\
1,100 \\
1,100 \\
1,100\end{array}$ & $\begin{array}{l}491 \\
492 \\
492 \\
489 \\
493 \\
493\end{array}$ & $\begin{array}{l}7.5 \\
7.5 \\
7.4 \\
7.4 \\
7.4 \\
7.4\end{array}$ & $\begin{array}{l}24.7 \\
24.7 \\
24.7 \\
24.7 \\
24.7 \\
24.7\end{array}$ & $\begin{array}{l}-- \\
- \\
-- \\
- \\
-\end{array}$ & $\begin{array}{l}8.1 \\
8.0 \\
7.9 \\
8.2 \\
8.0 \\
8.0\end{array}$ & $\begin{array}{r}99 \\
98 \\
97 \\
100 \\
99 \\
98\end{array}$ \\
\hline
\end{tabular}


Table 4. Water-quality data for station 403115080371801, Ohio River at river mile 54.8, June to October, 1995, Continued.

[ft = feet; $\mu \mathrm{S} / \mathrm{cm}=$ microsiemens per centimeter; ${ }^{\circ} \mathrm{C}=$ degrees celsius; $\mathrm{mg} / \mathrm{L}=$ milligrams per liter; .. = data not collected]

\begin{tabular}{|c|c|c|c|c|c|c|c|c|c|}
\hline Date & Time & $\begin{array}{l}\text { Sampling } \\
\text { depth } \\
\text { (ft) }\end{array}$ & $\begin{array}{c}\text { Sample } \\
\text { location } \\
\text { (ft from } \\
\text { left bank) }\end{array}$ & $\begin{array}{l}\text { Specific } \\
\text { conduct- } \\
\text { ance } \\
(\mu \mathrm{s} / \mathrm{cm})\end{array}$ & $\begin{array}{l}\text { pH } \\
\text { (stan- } \\
\text { dard } \\
\text { units) }\end{array}$ & $\begin{array}{c}\text { Temper- } \\
\text { ature, } \\
\text { water } \\
\left({ }^{\circ} \mathrm{C}\right)\end{array}$ & $\begin{array}{l}\text { Trans- } \\
\text { parency } \\
\text { (Secchi } \\
\text { disk) } \\
\text { (Et) }\end{array}$ & $\begin{array}{l}\text { Dissolved } \\
\text { oxygen } \\
\text { (mg/L) }\end{array}$ & $\begin{array}{c}\text { Dissolved } \\
\text { oxygen } \\
\text { (percent } \\
\text { satura- } \\
\text { tion) }\end{array}$ \\
\hline
\end{tabular}

September

221539

221540

221540

$22 \quad 1541$

$22 \quad 1541$

221542

$\begin{array}{rr}1.0 & 1,500 \\ 2.8 & 1,500 \\ 5.1 & 1,500 \\ 10 & 1,500 \\ 15 & 1,500 \\ 16 & 1,500\end{array}$

October

031549

031549

$03 \quad 1550$

031551

$03 \quad 1552$

031553

031608

$03 \quad 1608$

031609

031610

031610

031611

$0.7 \quad 400$

$2.6 \quad 400$

$4.8 \quad 400$

10

15

16

\section{6}

2.8

10

15

18

$0.7 \quad 1,100$

$2.9 \quad 1,100$

$4.9 \quad 1,100$

$10 \quad 1,100$

15

1601

1601

1602

$160316 \quad 1,100$

1,100

031619

$0.61,500$

3. 1,500

$5.0 \quad 1,500$

1620

11

15

162

16

031622

$17 \quad 1510$

$$
0.8
$$

3.2

4.9

9.7

15

19

24

1,500
1,500

400

400

400

400

400

400

400

$\begin{array}{llllll}492 & 7.5 & 24.5 & - & 7.5 & 92 \\ 490 & 7.4 & 24.6 & -- & 7.5 & 91 \\ 491 & 7.4 & 24.6 & - & 7.5 & 91 \\ 490 & 7.4 & 24.6 & - & 7.4 & 90 \\ 489 & 7.4 & 24.6 & - & 7.3 & 90 \\ 488 & 7.4 & 24.6 & -- & 7.3 & 89\end{array}$

480

480

481

484

482

486

481

479

\section{8}

478

477

486

484

482

479

482

488

479

485

482

486

488

492

483
500

499

499

498

497

497

498
7.5

7.5

7.5

7.5

7.5

7.5

$7 \cdot 5$

7.5

7.5

7.5

7.5

7.5

7.5

7.5

7.5

$7 \cdot 5$

7.5

24.1

24.1

24.2

24.2

24.2

24.2

24.1

24.1

24.1

24.1

24.1

24.1

23.9

23.9

23.9

23.9

23.9

23.9

7.5

7.5

7.5

7.5

7.5

7.5

23.8

23.8

23.8

23.8

23.8

23.8

7.5

7.5

7.5

7.4

7.4

7.5

19.3

19.4

19.4

19.3

19.3

19.3

19.3 $\cdots$

8.4

8. $4 \quad 103$

8.4103

-. $8.4 \quad 103$

- $8.4 \quad 103$

. $8.4 \quad 103$

- 8.4103

- $8.5 \quad 104$

- $8.5 \quad 104$

- $8.6 \quad 105$

. $8.6 \quad 105$

- $8.6 \quad 105$

- $8.6 \quad 105$

- $8.6 \quad 105$

. $8.6 \quad 104$

- $8.5 \quad 104$

. $8.5 \quad 104$

$\begin{array}{lll}\ldots & 8.5 & 104 \\ \ldots & 8.5 & 104\end{array}$

- $8.6 \quad 105$

. $8.6 \quad 104$

. $8.6 \quad 105$
- 8.6

- $8.6 \quad 805$

- $8.6 \quad 105$

- $8.5 \quad 103$

- $8.5 \quad 104$

$\begin{array}{lll}- & 9.2 & 101\end{array}$

$\begin{array}{lll} & 9.2 & 101 \\ & 9.3 & 101\end{array}$

$\begin{array}{lll}3.5 & 9.2 & 101\end{array}$

-. 9.2101

- 9.2101

$\begin{array}{lll}- & 9.2 & 101\end{array}$
. 9.2101 
Table 4. Water-quality data for station 403115080371801, Ohio River at river mile 54.8, June to October, 1995, Continued.

[ft $=$ feet $; \mu S / \mathrm{cm}=$ microsiemens per centimeter; ${ }^{\circ} \mathrm{C}=$ degrees Celsius; $\mathrm{mg} / \mathrm{L}=$ militigrams per liter; $\cdots$ = data not collected]

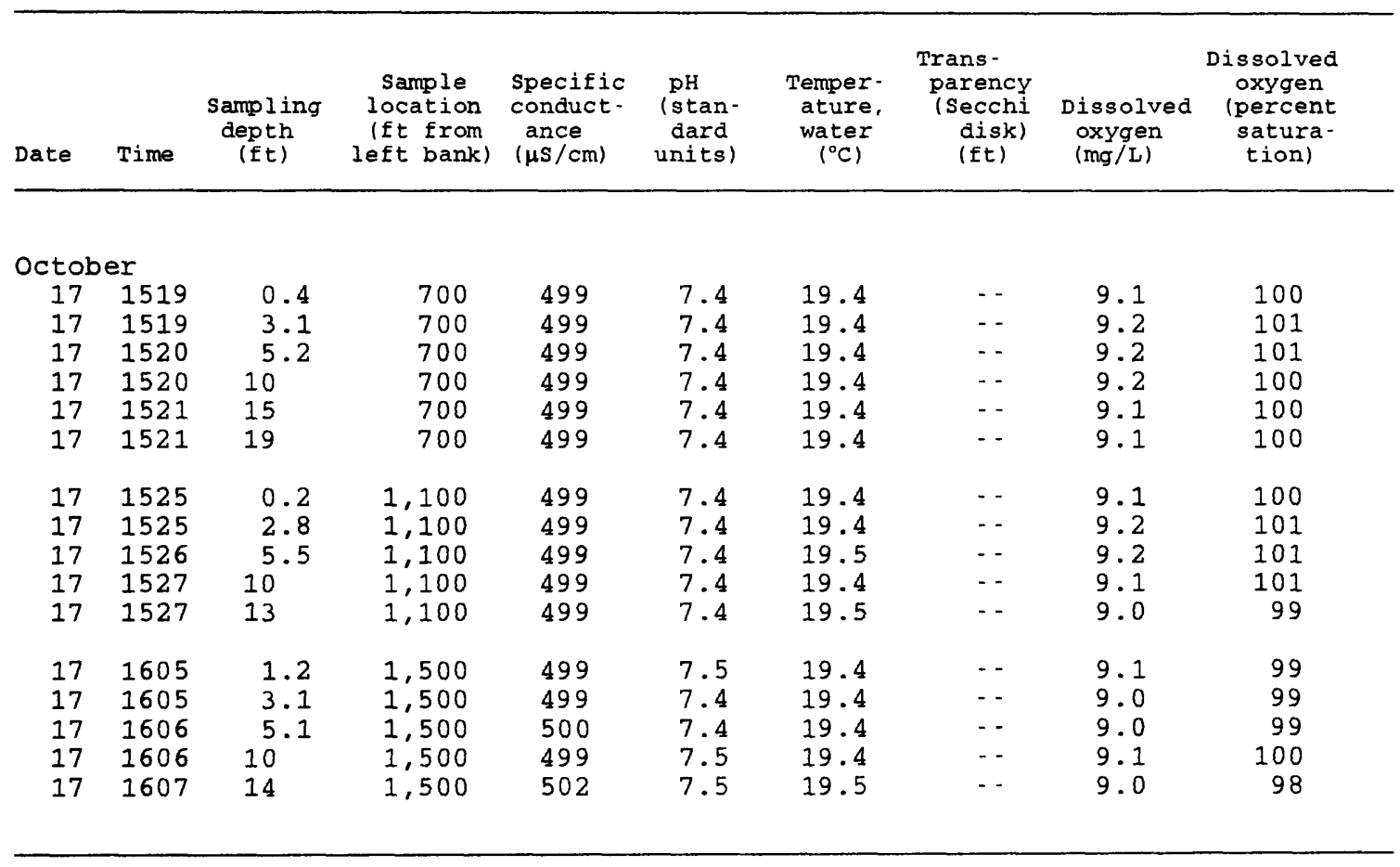


Table 5. Water-quality data for station 403045080370901, Ohio River at river mile 55.4, June to October, 1995.

[ft $=$ feet $; \mu \mathrm{S} / \mathrm{cm}=$ microsiemens per centimeter $;{ }^{\circ} \mathrm{C}=$ degrees Celsius : $\mathrm{mg} / \mathrm{L}=$ milligrams per liter; -. = data not collected]

\begin{tabular}{|c|c|c|c|c|c|c|c|c|c|}
\hline Date & Time & $\begin{array}{l}\text { Sampling } \\
\text { depth } \\
\text { (ft) }\end{array}$ & $\begin{array}{c}\text { Sample } \\
\text { location } \\
\text { (ft from } \\
\text { left bank) }\end{array}$ & $\begin{array}{l}\text { Specific } \\
\text { conduct- } \\
\text { ance } \\
(\mu \mathrm{S} / \mathrm{cm})\end{array}$ & $\begin{array}{l}\mathrm{pH} \\
\text { (stan- } \\
\text { dard } \\
\text { units) }\end{array}$ & $\begin{array}{c}\text { Temper- } \\
\text { ature, } \\
\text { water } \\
\left({ }^{\circ} \mathrm{C}\right)\end{array}$ & $\begin{array}{c}\text { Trans - } \\
\text { parency } \\
\text { (Secchi } \\
\text { disk) } \\
\text { (ft) }\end{array}$ & $\begin{array}{l}\text { Dissolved } \\
\text { oxygen } \\
(\mathrm{mg} / \mathrm{L})\end{array}$ & $\begin{array}{c}\text { Dissolved } \\
\text { oxygen } \\
\text { (percent } \\
\text { satura- } \\
\text { tion) }\end{array}$ \\
\hline
\end{tabular}

\begin{tabular}{|c|c|c|c|c|c|c|c|c|c|}
\hline $\begin{array}{r}\text { July } \\
11 \\
11 \\
11 \\
11\end{array}$ & $\begin{array}{l}1331 \\
1332 \\
1333 \\
1332\end{array}$ & $\begin{array}{r}0.3 \\
3.2 \\
8.3 \\
17\end{array}$ & $\begin{array}{l}700 \\
700 \\
700 \\
700\end{array}$ & $\begin{array}{l}429 \\
429 \\
429 \\
429\end{array}$ & $\begin{array}{l}7.4 \\
7.3 \\
7.3 \\
7.3\end{array}$ & $\begin{array}{l}27.9 \\
27.6 \\
27.5 \\
27.5\end{array}$ & $\begin{array}{l}- \\
- \\
- \\
-\end{array}$ & $\begin{array}{l}7.9 \\
7.9 \\
7.8 \\
7.8\end{array}$ & $\begin{array}{l}103 \\
102 \\
101 \\
101\end{array}$ \\
\hline $\begin{array}{l}25 \\
25 \\
25 \\
25\end{array}$ & $\begin{array}{l}1354 \\
1354 \\
1355 \\
1355\end{array}$ & $\begin{array}{r}0.3 \\
3.1 \\
9.2 \\
19\end{array}$ & $\begin{array}{l}700 \\
700 \\
700 \\
700\end{array}$ & $\begin{array}{l}427 \\
426 \\
428 \\
428\end{array}$ & $\begin{array}{l}7.6 \\
7.6 \\
7.5 \\
7.5\end{array}$ & $\begin{array}{l}30.4 \\
30.3 \\
29.9 \\
29.8\end{array}$ & $\begin{array}{l}-- \\
-- \\
--\end{array}$ & $\begin{array}{l}7.5 \\
7.5 \\
7.2 \\
7.2\end{array}$ & $\begin{array}{r}102 \\
102 \\
97 \\
97\end{array}$ \\
\hline $\begin{array}{r}\text { Augus } \\
08 \\
08 \\
08 \\
08\end{array}$ & $\begin{array}{l}0826 \\
0826 \\
0828 \\
0827\end{array}$ & $\begin{array}{l}0.4 \\
3.1 \\
12 \\
23\end{array}$ & $\begin{array}{l}700 \\
700 \\
700 \\
700\end{array}$ & $\begin{array}{l}452 \\
452 \\
450 \\
448\end{array}$ & $\begin{array}{l}7.5 \\
7.5 \\
7.5 \\
7.5\end{array}$ & $\begin{array}{l}28.8 \\
28.9 \\
28.9 \\
28.8\end{array}$ & $\begin{array}{l}-- \\
-- \\
--\end{array}$ & $\begin{array}{l}7.8 \\
7.8 \\
7.8 \\
7.7\end{array}$ & $\begin{array}{l}102 \\
103 \\
103 \\
101\end{array}$ \\
\hline $\begin{array}{l}22 \\
22 \\
22 \\
22\end{array}$ & $\begin{array}{l}1233 \\
1233 \\
1234 \\
1234\end{array}$ & $\begin{array}{r}0.7 \\
3.4 \\
8.6 \\
17\end{array}$ & $\begin{array}{l}700 \\
700 \\
700 \\
700\end{array}$ & $\begin{array}{l}482 \\
482 \\
481 \\
482\end{array}$ & $\begin{array}{l}7.6 \\
7.6 \\
7.6 \\
7.6\end{array}$ & $\begin{array}{l}32.4 \\
32.4 \\
32.4 \\
32.3\end{array}$ & $\begin{array}{l}\cdots \\
\cdots \\
\cdots\end{array}$ & $\begin{array}{l}7.6 \\
7.6 \\
7.5 \\
7.4\end{array}$ & $\begin{array}{l}106 \\
107 \\
105 \\
103\end{array}$ \\
\hline $\begin{array}{c}\text { Septe } \\
07 \\
07 \\
07 \\
07\end{array}$ & $\begin{array}{l}\text { nber } \\
1556 \\
1557 \\
1559 \\
1558\end{array}$ & $\begin{array}{l}0.2 \\
3.0 \\
10 \\
19\end{array}$ & $\begin{array}{l}700 \\
700 \\
700 \\
700\end{array}$ & $\begin{array}{l}480 \\
481 \\
481 \\
479\end{array}$ & $\begin{array}{l}8.3 \\
8.0 \\
7.8 \\
7.7\end{array}$ & $\begin{array}{l}29.6 \\
29.5 \\
29.3 \\
28.9\end{array}$ & $\begin{array}{l}-- \\
-- \\
--\end{array}$ & $\begin{array}{l}8.5 \\
7.7 \\
7.3 \\
6.9\end{array}$ & $\begin{array}{r}114 \\
104 \\
97 \\
92\end{array}$ \\
\hline $\begin{array}{l}22 \\
22 \\
22 \\
22\end{array}$ & $\begin{array}{l}1318 \\
1318 \\
1319 \\
1319\end{array}$ & $\begin{array}{r}0.9 \\
3.0 \\
8.6 \\
16\end{array}$ & $\begin{array}{l}700 \\
700 \\
700 \\
700\end{array}$ & $\begin{array}{l}492 \\
493 \\
493 \\
493\end{array}$ & $\begin{array}{l}7.4 \\
7.4 \\
7.4 \\
7.4\end{array}$ & $\begin{array}{l}24.6 \\
24.6 \\
24.6 \\
24.6\end{array}$ & $\begin{array}{l}\cdots \\
\cdots \\
-\end{array}$ & $\begin{array}{l}7.9 \\
7.8 \\
7.9 \\
8.0\end{array}$ & $\begin{array}{l}96 \\
96 \\
96 \\
98\end{array}$ \\
\hline $\begin{array}{c}\text { Octok } \\
03 \\
03 \\
03 \\
03\end{array}$ & $\begin{array}{l}\text { er } \\
1252 \\
1253 \\
1254 \\
1253\end{array}$ & $\begin{array}{r}0.3 \\
3.0 \\
7.9 \\
16\end{array}$ & $\begin{array}{l}700 \\
700 \\
700 \\
700\end{array}$ & $\begin{array}{l}478 \\
478 \\
477 \\
476\end{array}$ & $\begin{array}{l}7.5 \\
7.5 \\
7.5 \\
7.5\end{array}$ & $\begin{array}{l}24.2 \\
24.1 \\
23.9 \\
23.8\end{array}$ & $\begin{array}{l}- \\
\cdots \\
-\end{array}$ & $\begin{array}{l}8.5 \\
8.5 \\
8.3 \\
8.3\end{array}$ & $\begin{array}{l}104 \\
103 \\
101 \\
101\end{array}$ \\
\hline $\begin{array}{l}17 \\
17 \\
17 \\
17\end{array}$ & $\begin{array}{l}1259 \\
1300 \\
1301 \\
1300\end{array}$ & $\begin{array}{r}0.8 \\
3.3 \\
9.7 \\
20\end{array}$ & $\begin{array}{l}700 \\
700 \\
700 \\
700\end{array}$ & $\begin{array}{l}506 \\
507 \\
508 \\
504\end{array}$ & $\begin{array}{l}7.4 \\
7.4 \\
7.4 \\
7.4\end{array}$ & $\begin{array}{l}19.5 \\
19.5 \\
19.5 \\
19.5\end{array}$ & $\begin{array}{l}\cdots \\
\cdots \\
\cdots\end{array}$ & $\begin{array}{l}9.2 \\
9.2 \\
9.0 \\
9.0\end{array}$ & $\begin{array}{r}101 \\
101 \\
99 \\
99\end{array}$ \\
\hline
\end{tabular}


Table 6. Water-quality data for station 402930080363101, Ohio River at river mile 57.0, June to October, 1995.

[ft $=$ feet $\mu \mu \mathrm{s} / \mathrm{cm}=$ microsiemens per centimeter; ${ }^{\circ} \mathrm{C}=$ degrees Celsius; $\mathrm{mg} / \mathrm{L}=$ miligrams per liter; -- = data not collected]

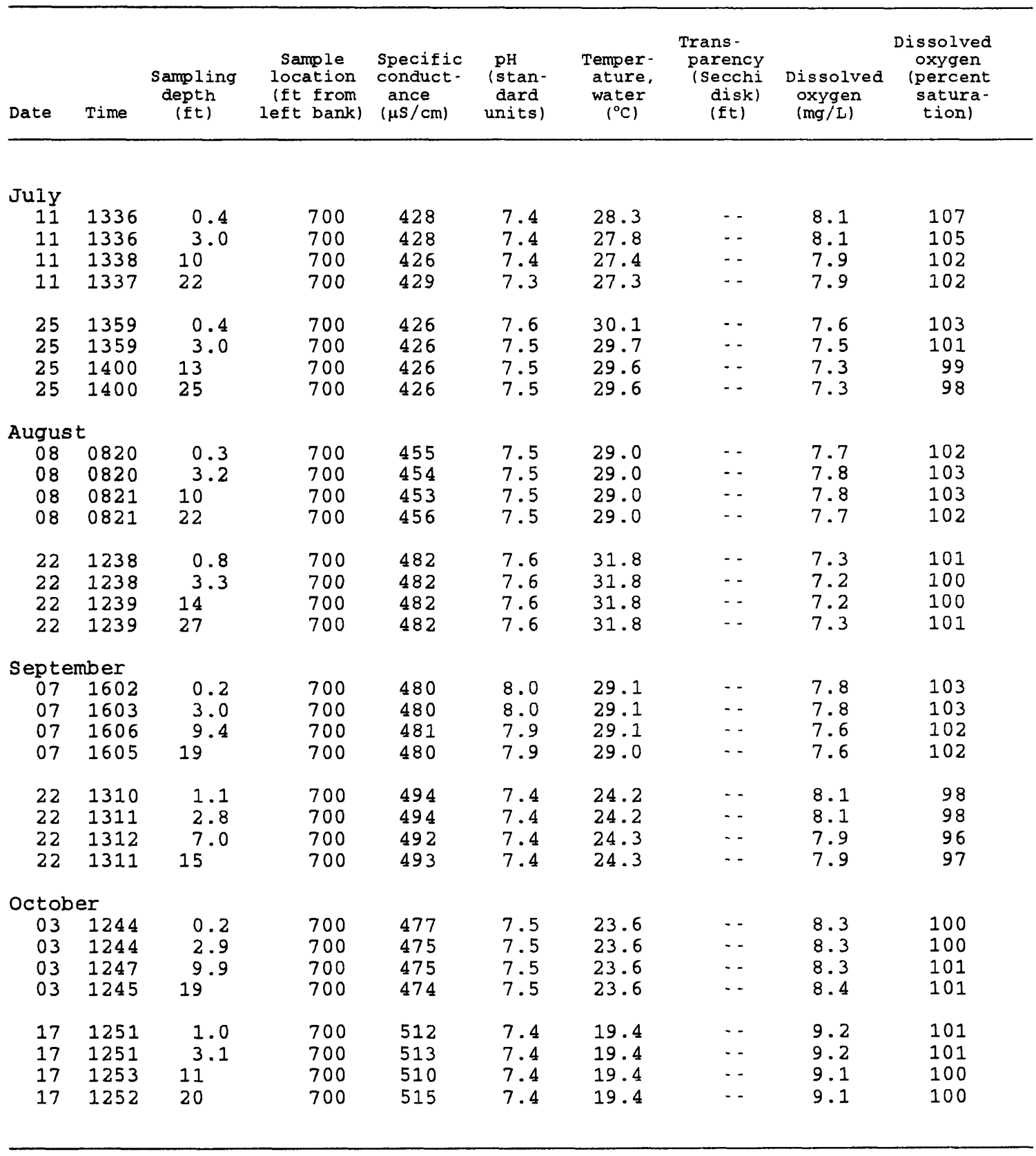


Table 7. Water-quality data for station 402654080361501, Ohio River at river mile 60.3, June to October, 1995.

$\left[\mathrm{ft}=\right.$ feet $; \mu \mathrm{s} / \mathrm{cm}=$ microsiemens per centimeter; ${ }^{\circ} \mathrm{C}=$ degrees Celsius;

$\mathrm{mg} / \mathrm{L}=\mathrm{mili}$ igrams per liter; $\cdots$ - = data not collected]

\begin{tabular}{|c|c|c|c|c|c|c|c|c|c|}
\hline Date & Time & $\begin{array}{l}\text { sampling } \\
\text { depth } \\
\text { (ft) }\end{array}$ & $\begin{array}{c}\text { Sample } \\
\text { location } \\
\text { (ft from } \\
\text { left bank) }\end{array}$ & $\begin{array}{l}\text { Specific } \\
\text { conduct- } \\
\text { ance } \\
(\mu \mathrm{s} / \mathrm{cm})\end{array}$ & $\begin{array}{l}\text { pH } \\
\text { (stan- } \\
\text { dard } \\
\text { units) }\end{array}$ & $\begin{array}{c}\text { Temper- } \\
\text { ature, } \\
\text { water } \\
\left({ }^{\circ} \mathrm{C}\right)\end{array}$ & $\begin{array}{l}\text { Trans- } \\
\text { parency } \\
\text { (Secchi } \\
\text { disk) } \\
\text { (ft) }\end{array}$ & $\begin{array}{l}\text { Dissolved } \\
\text { oxygen } \\
(\mathrm{mg} / \mathrm{L})\end{array}$ & $\begin{array}{c}\text { Dissolved } \\
\text { oxygen } \\
\text { (percent } \\
\text { satura- } \\
\text { tion) }\end{array}$ \\
\hline
\end{tabular}

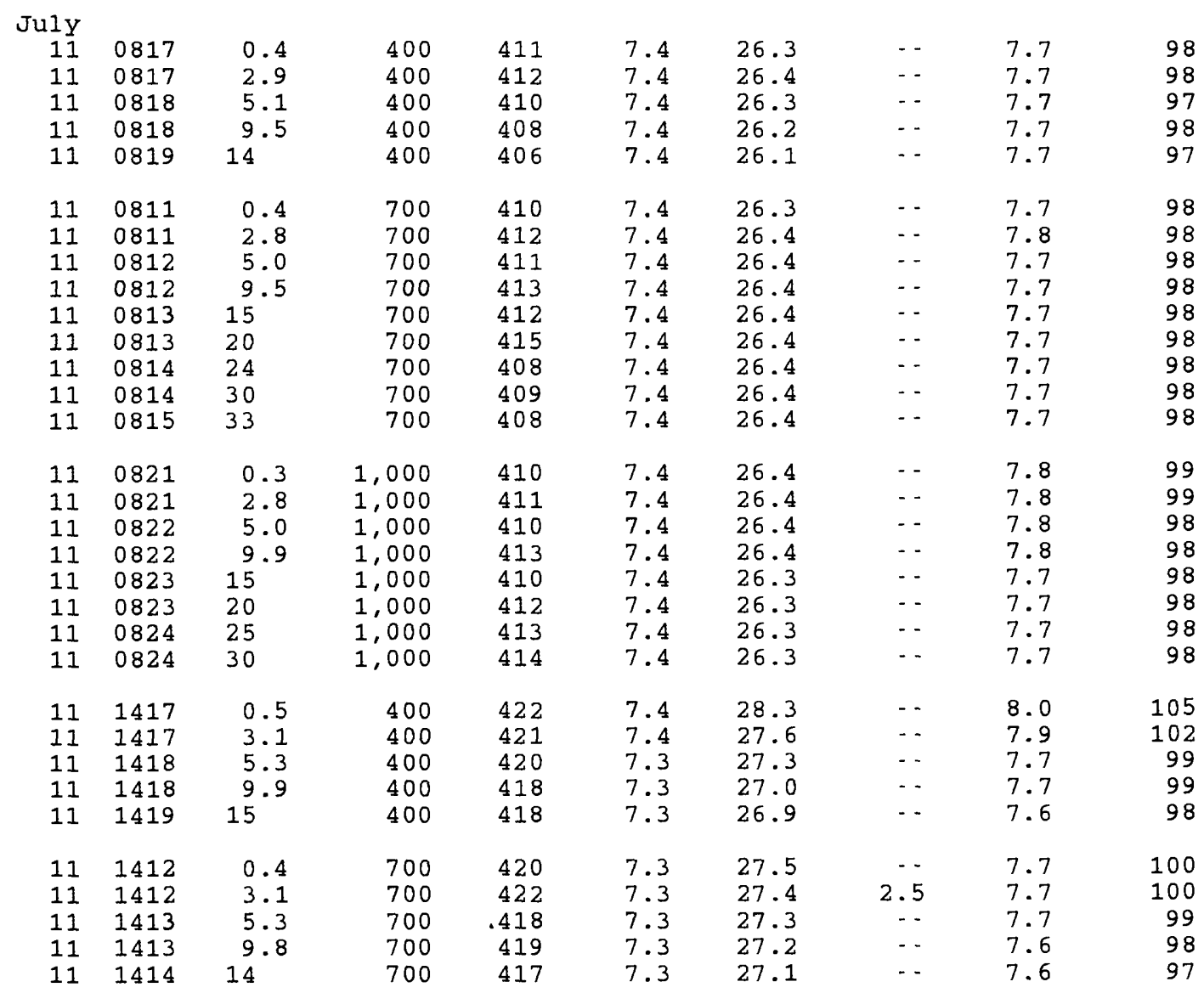


Table 7. Water-quality data for station 402654080361501 , Ohio River at river mile 60.3, June to October, 1995, Continued.

[ft $=$ feet $; \mu \mathrm{s} / \mathrm{cm}=$ microsiemens per centimeter $;{ }^{\circ} \mathrm{C}=$ degrees Celsius; $\mathrm{mg} / \mathrm{L}=$ milligrams per liter: - = data not collected]

\begin{tabular}{|c|c|c|c|c|c|c|c|c|c|}
\hline Date & Time & $\begin{array}{l}\text { Sampling } \\
\text { depth } \\
\text { (ft) }\end{array}$ & $\begin{array}{c}\text { Sample } \\
\text { location } \\
\text { (ft from } \\
\text { left bank) }\end{array}$ & $\begin{array}{l}\text { Specific } \\
\text { conduct- } \\
\text { ance } \\
(\mu \mathrm{s} / \mathrm{cm})\end{array}$ & $\begin{array}{l}\mathrm{pH} \\
\text { (stan- } \\
\text { dard } \\
\text { units) }\end{array}$ & $\begin{array}{c}\text { Temper- } \\
\text { ature, } \\
\text { water } \\
\left({ }^{\circ} \mathrm{C}\right)\end{array}$ & $\begin{array}{c}\text { Trans- } \\
\text { parency } \\
\text { (secchi } \\
\text { disk) } \\
\text { (ft) }\end{array}$ & $\begin{array}{l}\text { Dissolved } \\
\text { oxygen } \\
\text { (mg/L) }\end{array}$ & $\begin{array}{c}\text { Dissolved } \\
\text { oxygen } \\
\text { (percent } \\
\text { satura- } \\
\text { tion) }\end{array}$ \\
\hline
\end{tabular}

\begin{tabular}{|c|c|c|c|c|c|c|c|c|c|}
\hline $\begin{array}{r}\text { July } \\
11 \\
11 \\
11 \\
11 \\
11 \\
11 \\
11\end{array}$ & $\begin{array}{l}1407 \\
1407 \\
1408 \\
1408 \\
1409 \\
1409 \\
1410\end{array}$ & $\begin{array}{l}0.3 \\
3.2 \\
5.3 \\
9.8 \\
15 \\
20 \\
21\end{array}$ & $\begin{array}{l}1,000 \\
1,000 \\
1,000 \\
1,0000 \\
1,0000 \\
1,0000 \\
1,000\end{array}$ & $\begin{array}{l}424 \\
419 \\
423 \\
418 \\
416 \\
422 \\
418\end{array}$ & $\begin{array}{l}7.5 \\
7.4 \\
7.3 \\
7.3 \\
7.3 \\
7.3 \\
7.3\end{array}$ & $\begin{array}{l}28.5 \\
27.7 \\
27.3 \\
27.1 \\
27.1 \\
27.1 \\
27.0\end{array}$ & $\begin{array}{l}-- \\
-- \\
-- \\
-- \\
-- \\
--\end{array}$ & $\begin{array}{l}8.2 \\
8.0 \\
7.9 \\
7.7 \\
7.7 \\
7.7 \\
7.7\end{array}$ & $\begin{array}{r}108 \\
104 \\
101 \\
99 \\
99 \\
98 \\
98\end{array}$ \\
\hline $\begin{array}{l}25 \\
25 \\
25 \\
25 \\
25\end{array}$ & $\begin{array}{l}1518 \\
1519 \\
1519 \\
1520 \\
1520\end{array}$ & $\begin{array}{r}0.5 \\
3.1 \\
5.0 \\
9.9 \\
14\end{array}$ & $\begin{array}{l}400 \\
400 \\
400 \\
400 \\
400\end{array}$ & $\begin{array}{l}422 \\
423 \\
423 \\
423 \\
423\end{array}$ & $\begin{array}{l}7.4 \\
7.4 \\
7.4 \\
7.4 \\
7.4\end{array}$ & $\begin{array}{l}29.4 \\
29.4 \\
29.4 \\
29.4 \\
29.3\end{array}$ & $\begin{array}{l}\cdots \\
-- \\
\cdots \\
\cdots\end{array}$ & $\begin{array}{l}7.2 \\
7.1 \\
7.1 \\
7.0 \\
7.0\end{array}$ & $\begin{array}{l}96 \\
95 \\
94 \\
94 \\
93\end{array}$ \\
\hline $\begin{array}{l}25 \\
25 \\
25 \\
25 \\
25 \\
25\end{array}$ & $\begin{array}{l}1513 \\
1514 \\
1514 \\
1515 \\
1515 \\
1516\end{array}$ & $\begin{array}{l}0.6 \\
3.1 \\
5.1 \\
9.8 \\
15 \\
17\end{array}$ & $\begin{array}{l}700 \\
700 \\
700 \\
700 \\
700 \\
700\end{array}$ & $\begin{array}{l}420 \\
421 \\
421 \\
420 \\
425 \\
424\end{array}$ & $\begin{array}{l}7.4 \\
7.4 \\
7.4 \\
7.4 \\
7.4 \\
7.4\end{array}$ & $\begin{array}{l}29.4 \\
29.4 \\
29.4 \\
29.4 \\
29.4 \\
29.3\end{array}$ & $\begin{array}{r}-. \\
2.5 \\
-- \\
-- \\
- \\
--\end{array}$ & $\begin{array}{l}7.1 \\
7.1 \\
7.1 \\
7.1 \\
6.9 \\
6.9\end{array}$ & $\begin{array}{l}94 \\
95 \\
95 \\
94 \\
93 \\
93\end{array}$ \\
\hline $\begin{array}{l}25 \\
25 \\
25 \\
25 \\
25 \\
25\end{array}$ & $\begin{array}{l}1507 \\
1507 \\
1508 \\
1509 \\
1509 \\
1510\end{array}$ & $\begin{array}{l}0.5 \\
3.0 \\
4.9 \\
9.9 \\
15 \\
20\end{array}$ & $\begin{array}{l}1,000 \\
1,000 \\
1,000 \\
1,000 \\
1,000 \\
1,000\end{array}$ & $\begin{array}{l}420 \\
421 \\
418 \\
419 \\
419 \\
426\end{array}$ & $\begin{array}{l}7.4 \\
7.4 \\
7.4 \\
7.4 \\
7.4 \\
7.4\end{array}$ & $\begin{array}{l}29.6 \\
29.6 \\
29.5 \\
29.4 \\
29.4 \\
29.4\end{array}$ & $\begin{array}{l}- \\
-- \\
-- \\
-- \\
-\end{array}$ & $\begin{array}{l}7.4 \\
7.4 \\
7.3 \\
7.1 \\
7.0 \\
7.0\end{array}$ & $\begin{array}{r}100 \\
99 \\
97 \\
95 \\
94 \\
94\end{array}$ \\
\hline $\begin{array}{c}\text { Augus } \\
08 \\
08 \\
08 \\
08 \\
08\end{array}$ & $\begin{array}{l}0620 \\
0620 \\
0621 \\
0621 \\
0622\end{array}$ & $\begin{array}{c}0.2 \\
3.1 \\
5.0 \\
10 \\
14\end{array}$ & $\begin{array}{l}400 \\
400 \\
400 \\
400 \\
400\end{array}$ & $\begin{array}{l}473 \\
472 \\
473 \\
473 \\
471\end{array}$ & $\begin{array}{l}7.5 \\
7.5 \\
7.5 \\
7.5 \\
7.5\end{array}$ & $\begin{array}{l}28.6 \\
28.7 \\
28.7 \\
28.7 \\
28.7\end{array}$ & $\begin{array}{l}-- \\
-- \\
-- \\
--\end{array}$ & $\begin{array}{l}7.8 \\
7.8 \\
7.7 \\
7.8 \\
7.7\end{array}$ & $\begin{array}{l}102 \\
102 \\
102 \\
103 \\
101\end{array}$ \\
\hline $\begin{array}{l}08 \\
08 \\
08 \\
08 \\
08\end{array}$ & $\begin{array}{l}0624 \\
0624 \\
0625 \\
0625 \\
0626\end{array}$ & $\begin{array}{c}0.2 \\
3.3 \\
5.1 \\
10 \\
14\end{array}$ & $\begin{array}{l}700 \\
700 \\
700 \\
700 \\
700\end{array}$ & $\begin{array}{l}471 \\
470 \\
472 \\
473 \\
470\end{array}$ & $\begin{array}{l}7.5 \\
7.5 \\
7.5 \\
7.5 \\
7.5\end{array}$ & $\begin{array}{l}28.8 \\
28.9 \\
28.9 \\
28.9 \\
28.9\end{array}$ & $\begin{array}{l}-- \\
-- \\
-- \\
--\end{array}$ & $\begin{array}{l}7.9 \\
7.8 \\
7.8 \\
7.8 \\
7.8\end{array}$ & $\begin{array}{l}104 \\
103 \\
103 \\
102 \\
103\end{array}$ \\
\hline
\end{tabular}


Table 7. Water-quality data for station 402654080361501 , Ohio River at river mile 60.3, June to October, 1995, Continued.

[ft $=$ feet $; \mu \mathrm{S} / \mathrm{cm}=$ microsiemens per centimeter; ${ }^{\circ} \mathrm{C}=$ degrees Celsius;

$\mathrm{mg} / \mathrm{L}=$ miliigrams per liter; $\cdots$ = data not collected]

\begin{tabular}{|c|c|c|c|c|c|c|c|c|c|}
\hline Date & Time & $\begin{array}{l}\text { Sampling } \\
\text { depth } \\
\text { (Et) }\end{array}$ & $\begin{array}{c}\text { Sample } \\
\text { location } \\
\text { (Et from } \\
\text { left bank) }\end{array}$ & 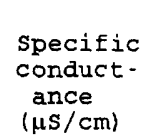 & $\begin{array}{l}\mathrm{pH} \\
\text { (stan- } \\
\text { dard } \\
\text { units) }\end{array}$ & $\begin{array}{c}\text { Temper- } \\
\text { ature, } \\
\text { water } \\
\left({ }^{\circ} \mathrm{C}\right\rangle\end{array}$ & $\begin{array}{l}\text { Trans- } \\
\text { parency } \\
\text { (Secchi } \\
\text { disk) } \\
\text { (ft) }\end{array}$ & $\begin{array}{l}\text { Dissolved } \\
\text { oxygen } \\
(\mathrm{mg} / \mathrm{L})\end{array}$ & $\begin{array}{c}\text { Dissolved } \\
\text { oxygen } \\
\text { (percent } \\
\text { satura- } \\
\text { tion) }\end{array}$ \\
\hline
\end{tabular}

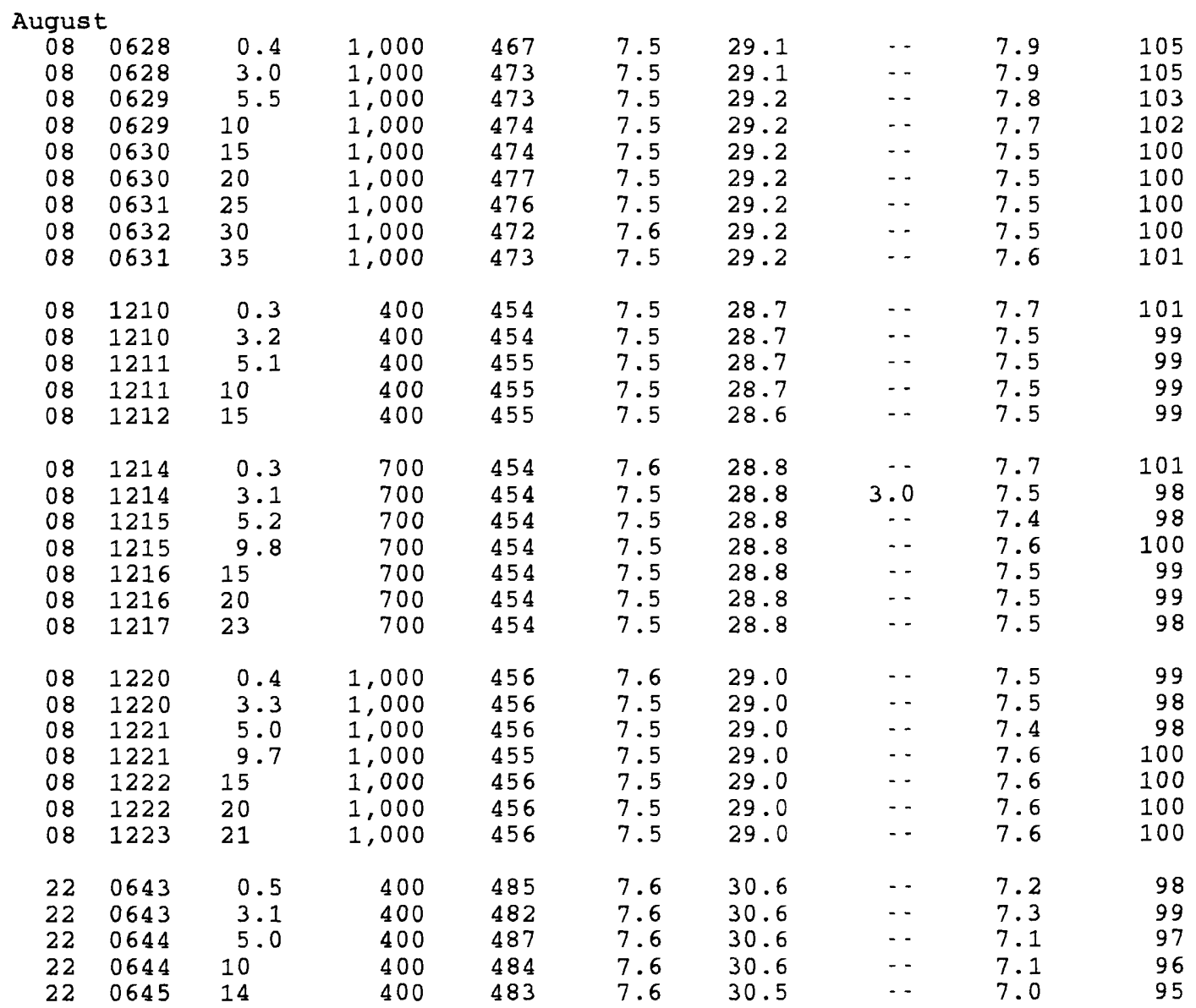


Table 7. Water-quality data for station 402654080361501, Ohio River at river mile 60.3, June to October, 1995, Continued.

[ft $=$ feet $; \mu \mathrm{s} / \mathrm{cm}=$ microsiemens per centimeter; ${ }^{\circ} \mathrm{C}=$ degrees Celsius; $\mathrm{mg} / \mathrm{L}=\mathrm{milligrams}$ per liter;.- = data not collected]

\begin{tabular}{|c|c|c|c|c|c|c|c|c|c|}
\hline Date & Time & $\begin{array}{l}\text { Sampling } \\
\text { depth } \\
\text { (ft) }\end{array}$ & $\begin{array}{c}\text { Sample } \\
\text { location } \\
\text { (ft from } \\
\text { left bank) }\end{array}$ & $\begin{array}{l}\text { Specific } \\
\text { conduct- } \\
\text { ance } \\
(\mu \mathrm{s} / \mathrm{cm})\end{array}$ & $\begin{array}{l}\text { pH } \\
\text { (stan- } \\
\text { dard } \\
\text { units) }\end{array}$ & $\begin{array}{c}\text { Temper- } \\
\text { ature, } \\
\text { water } \\
\left({ }^{\circ} \mathrm{C}\right)\end{array}$ & $\begin{array}{l}\text { Trans- } \\
\text { parency } \\
\text { (Secchi } \\
\text { disk) } \\
\text { (ft) }\end{array}$ & $\begin{array}{l}\text { Dissolved } \\
\text { oxygen } \\
(\mathrm{mg} / \mathrm{L})\end{array}$ & $\begin{array}{c}\text { Dissolved } \\
\text { oxygen } \\
\text { (percent } \\
\text { satura- } \\
\text { tion) }\end{array}$ \\
\hline
\end{tabular}

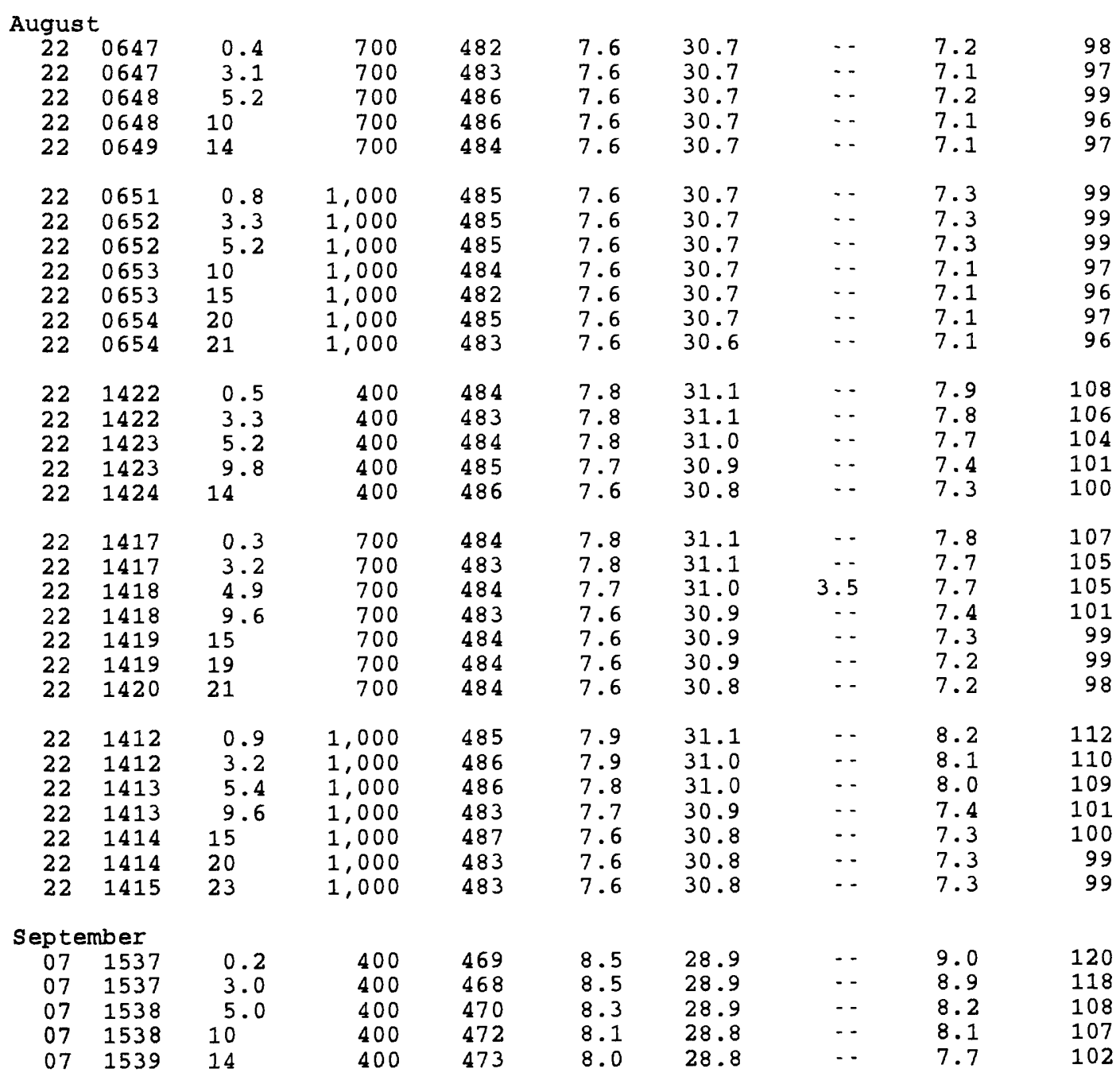


Table 7. Water-quality data for station 402654080361501, Ohio River at river mile 60.3, June to October, 1995, Continued.

[ft $=$ feet $; \mu \mathrm{s} / \mathrm{cm}=$ microsiemens per centimeter; ${ }^{\circ} \mathrm{C}=$ degrees Celsius;

$\mathrm{mg} / \mathrm{L}=$ milijgrams per liter; $\cdots$ = data not collected]

\begin{tabular}{|c|c|c|c|c|c|c|c|c|c|}
\hline Date & Time & $\begin{array}{l}\text { Sampling } \\
\text { depth } \\
\text { (ft) }\end{array}$ & $\begin{array}{c}\text { sample } \\
\text { location } \\
\text { (ft from } \\
\text { left bank) }\end{array}$ & $\begin{array}{l}\text { Specific } \\
\text { conduct - } \\
\text { ance } \\
(\mu \mathrm{s} / \mathrm{cm})\end{array}$ & $\begin{array}{l}\mathrm{pH} \\
\text { (stan- } \\
\text { dard } \\
\text { units) }\end{array}$ & $\begin{array}{c}\text { Temper- } \\
\text { ature, } \\
\text { water } \\
\left({ }^{\circ} \mathrm{C}\right)\end{array}$ & $\begin{array}{l}\text { Trans- } \\
\text { parency } \\
\text { (Secchi } \\
\text { disk) } \\
\text { (ft) }\end{array}$ & $\begin{array}{l}\text { Dissolved } \\
\text { oxygen } \\
\text { (mg/L) }\end{array}$ & $\begin{array}{c}\text { Dissolve } \\
\text { oxygen } \\
\text { (percen } \\
\text { satura } \\
\text { tion) }\end{array}$ \\
\hline \multicolumn{10}{|c|}{ September } \\
\hline $\begin{array}{l}07 \\
07 \\
07 \\
07 \\
07\end{array}$ & $\begin{array}{l}1531 \\
1531 \\
1532 \\
1532 \\
1533\end{array}$ & $\begin{array}{l}0.2 \\
3.0 \\
5.0 \\
10 \\
15\end{array}$ & $\begin{array}{l}700 \\
700 \\
700 \\
700 \\
700\end{array}$ & $\begin{array}{l}470 \\
469 \\
468 \\
469 \\
472\end{array}$ & $\begin{array}{l}8.4 \\
8.4 \\
8.3 \\
8.2 \\
8.0\end{array}$ & $\begin{array}{l}28.9 \\
28.9 \\
28.9 \\
28.9 \\
28.8\end{array}$ & $\begin{array}{r}-- \\
2.0 \\
-- \\
-- \\
--\end{array}$ & $\begin{array}{l}8.5 \\
8.6 \\
8.4 \\
8.1 \\
7.7\end{array}$ & $\begin{array}{l}114 \\
115 \\
112 \\
108 \\
103\end{array}$ \\
\hline $\begin{array}{l}07 \\
07 \\
07 \\
07 \\
07 \\
07 \\
07\end{array}$ & $\begin{array}{l}1525 \\
1525 \\
1526 \\
1526 \\
1527 \\
1527 \\
1528\end{array}$ & $\begin{array}{l}0.2 \\
3.0 \\
5.0 \\
10 \\
15 \\
20 \\
25\end{array}$ & $\begin{array}{l}1,000 \\
1,000 \\
1,000 \\
1,000 \\
1,0000 \\
1,000 \\
1,000\end{array}$ & $\begin{array}{l}468 \\
471 \\
470 \\
470 \\
470 \\
468 \\
469\end{array}$ & $\begin{array}{l}8.5 \\
8.4 \\
8.4 \\
8.1 \\
8.1 \\
8.1 \\
8.1\end{array}$ & $\begin{array}{l}28.9 \\
28.9 \\
28.9 \\
28.8 \\
28.8 \\
28.8 \\
28.8\end{array}$ & $\begin{array}{l}-- \\
\cdots \\
\cdots \\
\cdots \\
\cdots \\
-\end{array}$ & $\begin{array}{l}8.7 \\
8.6 \\
8.6 \\
7.8 \\
7.8 \\
7.8 \\
7.6\end{array}$ & $\begin{array}{l}115 \\
115 \\
115 \\
103 \\
103 \\
103 \\
101\end{array}$ \\
\hline $\begin{array}{l}08 \\
08 \\
08 \\
08 \\
08\end{array}$ & $\begin{array}{l}0622 \\
0622 \\
0623 \\
0623 \\
0624\end{array}$ & $\begin{array}{l}0.2 \\
3.0 \\
5.0 \\
10 \\
15\end{array}$ & $\begin{array}{l}400 \\
400 \\
400 \\
400 \\
400\end{array}$ & $\begin{array}{l}481 \\
483 \\
485 \\
481 \\
480\end{array}$ & $\begin{array}{l}7.7 \\
7.7 \\
7.7 \\
7.7 \\
7.7\end{array}$ & $\begin{array}{l}28.7 \\
28.8 \\
28.8 \\
28.8 \\
28.8\end{array}$ & $\begin{array}{l}\cdots \\
\cdots \\
\cdots \\
\cdots\end{array}$ & $\begin{array}{l}7.0 \\
7.3 \\
7.3 \\
7.2 \\
7.1\end{array}$ & $\begin{array}{l}93 \\
97 \\
96 \\
96 \\
94\end{array}$ \\
\hline $\begin{array}{l}08 \\
08 \\
08 \\
08 \\
08\end{array}$ & $\begin{array}{l}0626 \\
0626 \\
0627 \\
0627 \\
0628\end{array}$ & $\begin{array}{l}0.2 \\
3.0 \\
5.0 \\
10 \\
14\end{array}$ & $\begin{array}{l}700 \\
700 \\
700 \\
700 \\
700\end{array}$ & $\begin{array}{l}483 \\
482 \\
482 \\
483 \\
484\end{array}$ & $\begin{array}{l}7.7 \\
7.7 \\
7.7 \\
7.7 \\
7.7\end{array}$ & $\begin{array}{l}28.7 \\
28.8 \\
28.8 \\
28.8 \\
28.8\end{array}$ & $\begin{array}{l}\cdots \\
\cdots \\
\cdots \\
\cdots\end{array}$ & $\begin{array}{l}7.4 \\
7.4 \\
7.4 \\
7.2 \\
7.4\end{array}$ & $\begin{array}{l}98 \\
98 \\
98 \\
96 \\
98\end{array}$ \\
\hline $\begin{array}{l}08 \\
08 \\
08 \\
08 \\
08\end{array}$ & $\begin{array}{l}0630 \\
0630 \\
0631 \\
0631 \\
0632\end{array}$ & $\begin{array}{l}0.2 \\
3.0 \\
5.0 \\
10 \\
13\end{array}$ & $\begin{array}{l}1,000 \\
1,000 \\
1,000 \\
1,000 \\
1,000\end{array}$ & $\begin{array}{l}480 \\
484 \\
478 \\
482 \\
485\end{array}$ & $\begin{array}{l}7.8 \\
7.8 \\
7.8 \\
7.8 \\
7.8\end{array}$ & $\begin{array}{l}28.6 \\
28.7 \\
28.7 \\
28.7 \\
28.7\end{array}$ & $\begin{array}{l}-- \\
\cdots \\
\cdots \\
\cdots\end{array}$ & $\begin{array}{l}7.5 \\
7.5 \\
7.5 \\
7.5 \\
7.5\end{array}$ & $\begin{array}{l}99 \\
99 \\
99 \\
99 \\
99\end{array}$ \\
\hline $\begin{array}{l}22 \\
22 \\
22 \\
22 \\
22\end{array}$ & $\begin{array}{l}1238 \\
1238 \\
1239 \\
1239 \\
1240\end{array}$ & $\begin{array}{r}0.6 \\
2.7 \\
5.0 \\
9.9 \\
14\end{array}$ & $\begin{array}{l}400 \\
400 \\
400 \\
400 \\
400\end{array}$ & $\begin{array}{l}497 \\
497 \\
498 \\
498 \\
497\end{array}$ & $\begin{array}{l}7.4 \\
7.4 \\
7.4 \\
7.4 \\
7.4\end{array}$ & $\begin{array}{l}24.6 \\
24.6 \\
24.6 \\
24.6 \\
24.6\end{array}$ & $\begin{array}{l}\cdots \\
\cdots \\
\cdots \\
\cdots\end{array}$ & $\begin{array}{l}7.6 \\
7.6 \\
7.6 \\
7.5 \\
7.5\end{array}$ & $\begin{array}{l}93 \\
92 \\
93 \\
92 \\
91\end{array}$ \\
\hline
\end{tabular}


Table 7. Water-quality data for station 402654080361501, Ohio River at river mile 60.3, June to October, 1995, Continued.

[ft $=$ feet $; \mu \mathrm{s} / \mathrm{cm}=$ microsiemens per centimeter; ${ }^{\circ} \mathrm{C}=$ degrees Celsius;

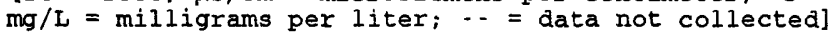

\begin{tabular}{|c|c|c|c|c|c|c|c|c|c|}
\hline Date & Time & $\begin{array}{l}\text { Sampling } \\
\text { depth } \\
\text { (ft) }\end{array}$ & $\begin{array}{c}\text { Sample } \\
\text { location } \\
\text { (ft from } \\
\text { left bank) }\end{array}$ & $\begin{array}{l}\text { Specific } \\
\text { conduct- } \\
\text { ance } \\
(\mu \mathrm{s} / \mathrm{cm})\end{array}$ & $\begin{array}{l}\text { pH } \\
\text { (stan- } \\
\text { dard } \\
\text { units) }\end{array}$ & $\begin{array}{c}\text { Temper- } \\
\text { ature, } \\
\text { water } \\
\left({ }^{\circ} \mathrm{C}\right)\end{array}$ & $\begin{array}{l}\text { Trans- } \\
\text { parency } \\
\text { (Secchi } \\
\text { disk) } \\
\text { (ft) }\end{array}$ & $\begin{array}{l}\text { Dissolved } \\
\text { oxygen } \\
(\mathrm{mg} / \mathrm{L})\end{array}$ & $\begin{array}{c}\text { Dissolved } \\
\text { oxygen } \\
\text { (percent } \\
\text { satura- } \\
\text { tion) }\end{array}$ \\
\hline
\end{tabular}

\begin{tabular}{|c|c|c|c|c|c|c|c|c|c|}
\hline \multicolumn{10}{|c|}{ september } \\
\hline $\begin{array}{l}22 \\
22 \\
22 \\
22 \\
22\end{array}$ & $\begin{array}{l}1244 \\
1244 \\
1245 \\
1245 \\
1246\end{array}$ & $\begin{array}{l}1.3 \\
3.1 \\
5.2 \\
10 \\
15\end{array}$ & $\begin{array}{l}700 \\
700 \\
700 \\
700 \\
700\end{array}$ & $\begin{array}{l}499 \\
498 \\
500 \\
498 \\
496\end{array}$ & $\begin{array}{l}7.4 \\
7.4 \\
7.4 \\
7.4 \\
7.4\end{array}$ & $\begin{array}{l}24.7 \\
24.7 \\
24.7 \\
24.7 \\
24.7\end{array}$ & $\begin{array}{l}-- \\
- \\
- \\
- \\
-\end{array}$ & $\begin{array}{l}7.5 \\
7.6 \\
7.6 \\
7.5 \\
7.5\end{array}$ & $\begin{array}{l}92 \\
93 \\
93 \\
92 \\
92\end{array}$ \\
\hline $\begin{array}{l}22 \\
22 \\
22 \\
22 \\
22 \\
22\end{array}$ & $\begin{array}{l}1248 \\
1248 \\
1249 \\
1249 \\
1250 \\
1250\end{array}$ & $\begin{array}{r}0.6 \\
3.1 \\
5.0 \\
9.6 \\
15 \\
20\end{array}$ & $\begin{array}{l}1,000 \\
1,000 \\
1,000 \\
1,000 \\
1,000 \\
1,000\end{array}$ & $\begin{array}{l}500 \\
496 \\
498 \\
501 \\
501 \\
499\end{array}$ & $\begin{array}{l}7.4 \\
7.4 \\
7.4 \\
7.4 \\
7.4 \\
7.4\end{array}$ & $\begin{array}{l}24.7 \\
24.7 \\
24.7 \\
24.7 \\
24.7 \\
24.6\end{array}$ & $\begin{array}{l}-- \\
-- \\
-- \\
-- \\
-- \\
--\end{array}$ & $\begin{array}{l}7.5 \\
7.5 \\
7.5 \\
7.5 \\
7.5 \\
7.5\end{array}$ & $\begin{array}{l}92 \\
92 \\
92 \\
92 \\
92 \\
92\end{array}$ \\
\hline $\begin{array}{c}\text { Octob } \\
03 \\
03 \\
03 \\
03 \\
03\end{array}$ & $\begin{array}{l}\text { er } \\
1219 \\
1219 \\
1220 \\
1220 \\
1221\end{array}$ & $\begin{array}{r}0.5 \\
2.8 \\
5.1 \\
10 \\
14\end{array}$ & 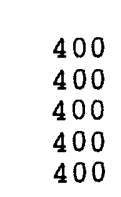 & $\begin{array}{l}476 \\
476 \\
475 \\
472 \\
481\end{array}$ & $\begin{array}{l}7.5 \\
7.5 \\
7.5 \\
7.5 \\
7.5\end{array}$ & $\begin{array}{l}23.2 \\
23.1 \\
23.1 \\
23.0 \\
23.0\end{array}$ & $\begin{array}{l}-- \\
\cdots \\
\cdots \\
- \\
-\end{array}$ & $\begin{array}{l}8.4 \\
8.4 \\
8.4 \\
8.3 \\
8.3\end{array}$ & $\begin{array}{l}100 \\
101 \\
101 \\
100 \\
100\end{array}$ \\
\hline $\begin{array}{l}03 \\
03 \\
03 \\
03 \\
03 \\
03 \\
03 \\
03\end{array}$ & $\begin{array}{l}1224 \\
1224 \\
1225 \\
1225 \\
1226 \\
1226 \\
1227 \\
1227\end{array}$ & $\begin{array}{c}0.3 \\
2.7 \\
4.7 \\
9.4 \\
15 \\
19 \\
24 \\
30\end{array}$ & $\begin{array}{l}700 \\
700 \\
700 \\
700 \\
700 \\
700 \\
700 \\
700\end{array}$ & $\begin{array}{l}475 \\
477 \\
477 \\
478 \\
472 \\
479 \\
473 \\
481\end{array}$ & $\begin{array}{l}7.5 \\
7.5 \\
7.5 \\
7.5 \\
7.5 \\
7.5 \\
7.5 \\
7.5\end{array}$ & $\begin{array}{l}23.1 \\
23.1 \\
23.1 \\
23.0 \\
23.0 \\
23.0 \\
23.0 \\
23.0\end{array}$ & $\begin{array}{r}\cdots \\
\cdots \\
3.5 \\
-- \\
- \\
- \\
- \\
-\end{array}$ & $\begin{array}{l}8.3 \\
8.4 \\
8.3 \\
8.4 \\
8.3 \\
8.4 \\
8.3 \\
8.3\end{array}$ & $\begin{array}{r}99 \\
100 \\
100 \\
100 \\
100 \\
100 \\
100 \\
99\end{array}$ \\
\hline $\begin{array}{l}03 \\
03 \\
03 \\
03 \\
03 \\
03 \\
03 \\
03\end{array}$ & $\begin{array}{l}1229 \\
1229 \\
1230 \\
1231 \\
1231 \\
1232 \\
1232 \\
1233\end{array}$ & $\begin{array}{c}0.2 \\
2.9 \\
4.8 \\
9.5 \\
15 \\
20 \\
25 \\
29\end{array}$ & $\begin{array}{l}1,000 \\
1,000 \\
1,000 \\
1,000 \\
1,000 \\
1,000 \\
1,000 \\
1,000\end{array}$ & $\begin{array}{l}475 \\
476 \\
474 \\
474 \\
478 \\
477 \\
474 \\
477\end{array}$ & $\begin{array}{l}7.5 \\
7.5 \\
7.5 \\
7.5 \\
7.5 \\
7.5 \\
7.5 \\
7.5\end{array}$ & $\begin{array}{l}23.2 \\
23.2 \\
23.1 \\
23.0 \\
23.0 \\
23.0 \\
23.0 \\
23.0\end{array}$ & $\begin{array}{l}- \\
- \\
- \\
- \\
- \\
\cdots \\
- \\
-\end{array}$ & $\begin{array}{l}8.5 \\
8.4 \\
8.5 \\
8.4 \\
8.4 \\
8.4 \\
8.3 \\
8.3\end{array}$ & $\begin{array}{l}101 \\
100 \\
102 \\
100 \\
100 \\
100 \\
99 \\
99\end{array}$ \\
\hline $\begin{array}{l}17 \\
17 \\
17 \\
17 \\
17\end{array}$ & $\begin{array}{l}1238 \\
1238 \\
1239 \\
1239 \\
1240\end{array}$ & $\begin{array}{l}0.9 \\
3.2 \\
5.5 \\
10 \\
14\end{array}$ & $\begin{array}{l}400 \\
400 \\
400 \\
400 \\
400\end{array}$ & $\begin{array}{l}519 \\
519 \\
517 \\
520 \\
519\end{array}$ & $\begin{array}{l}7.4 \\
7.4 \\
7.4 \\
7.4 \\
7.4\end{array}$ & $\begin{array}{l}19.7 \\
19.7 \\
19.6 \\
19.6 \\
19.6\end{array}$ & $\begin{array}{l}-- \\
\ldots \\
- \\
- \\
-\end{array}$ & $\begin{array}{l}8.8 \\
8.8 \\
8.8 \\
8.9 \\
8.9\end{array}$ & $\begin{array}{l}97 \\
97 \\
97 \\
97 \\
98\end{array}$ \\
\hline
\end{tabular}


Table 7. Water-quality data for station 402654080361501, Ohio River at river mile 60.3, June to october, 1995, Continued.

[ft $=$ feet; $\mu \mathrm{S} / \mathrm{cm}=$ microsiemens per centimeter; ${ }^{\circ} \mathrm{C}=$ degrees Celsius ; $\mathrm{mg} / \mathrm{L}=\mathrm{milligrams}$ per liter;.. = data not collected]

\begin{tabular}{|c|c|c|c|c|c|c|c|c|c|}
\hline Date & Time & $\begin{array}{l}\text { Sampling } \\
\text { depth } \\
\text { (ft) }\end{array}$ & $\begin{array}{c}\text { Sample } \\
\text { location } \\
\text { (ft from } \\
\text { left bank) }\end{array}$ & $\begin{array}{l}\text { Specific } \\
\text { conduct. } \\
\text { ance } \\
(\mu \mathrm{S} / \mathrm{cm})\end{array}$ & $\begin{array}{l}\mathrm{pH} \\
\text { (stan- } \\
\text { dard } \\
\text { units) }\end{array}$ & $\begin{array}{c}\text { Temper- } \\
\text { ature, } \\
\text { water } \\
\left({ }^{\circ} \mathrm{C}\right)\end{array}$ & $\begin{array}{l}\text { Trans- } \\
\text { parency } \\
\text { (Secchi } \\
\text { disk) } \\
\text { (ft) }\end{array}$ & $\begin{array}{l}\text { Dissolved } \\
\text { oxygen } \\
(\mathrm{mg} / \mathrm{L})\end{array}$ & $\begin{array}{c}\text { Dissolved } \\
\text { oxygen } \\
\text { (percent } \\
\text { satura- } \\
\text { tion) }\end{array}$ \\
\hline \multicolumn{10}{|c|}{ October } \\
\hline $\begin{array}{l}17 \\
17 \\
17 \\
17 \\
17 \\
17 \\
17 \\
17 \\
17\end{array}$ & $\begin{array}{l}1231 \\
1232 \\
1232 \\
1233 \\
1233 \\
1234 \\
1235 \\
1235 \\
1236\end{array}$ & $\begin{array}{c}0.6 \\
3.1 \\
5.0 \\
10 \\
15 \\
20 \\
25 \\
30 \\
32\end{array}$ & $\begin{array}{l}700 \\
700 \\
700 \\
700 \\
700 \\
700 \\
700 \\
700 \\
700\end{array}$ & $\begin{array}{l}519 \\
517 \\
518 \\
518 \\
518 \\
520 \\
517 \\
521 \\
522\end{array}$ & $\begin{array}{l}7.4 \\
7.4 \\
7.4 \\
7.4 \\
7.4 \\
7.4 \\
7.4 \\
7.4 \\
7.4\end{array}$ & $\begin{array}{l}19.7 \\
19.7 \\
19.7 \\
19.7 \\
19.7 \\
19.7 \\
19.7 \\
19.7 \\
19.7\end{array}$ & $\begin{array}{c}- \\
- \\
3.5 \\
- \\
- \\
\cdots \\
- \\
- \\
-\end{array}$ & $\begin{array}{l}8.8 \\
8.8 \\
8.8 \\
8.7 \\
8.7 \\
8.7 \\
8.7 \\
8.7 \\
8.7\end{array}$ & $\begin{array}{l}97 \\
97 \\
97 \\
96 \\
96 \\
96 \\
96 \\
96 \\
96\end{array}$ \\
\hline $\begin{array}{l}17 \\
17 \\
17 \\
17 \\
17 \\
17 \\
17\end{array}$ & $\begin{array}{l}1242 \\
1242 \\
1243 \\
1243 \\
1244 \\
1244 \\
1245\end{array}$ & $\begin{array}{l}0.6 \\
3.2 \\
5.1 \\
10 \\
15 \\
20 \\
25\end{array}$ & $\begin{array}{l}1,000 \\
1,000 \\
1,000 \\
1,0000 \\
1,0000 \\
1,000 \\
1,000\end{array}$ & $\begin{array}{l}519 \\
519 \\
520 \\
518 \\
518 \\
520 \\
524\end{array}$ & $\begin{array}{l}7.4 \\
7.4 \\
7.4 \\
7.4 \\
7.4 \\
7.4 \\
7.4\end{array}$ & $\begin{array}{l}19.7 \\
19.7 \\
19.7 \\
19.7 \\
19.6 \\
19.6 \\
19.6\end{array}$ & $\begin{array}{l}-- \\
-- \\
-- \\
\cdots \\
- \\
\cdots \\
-\end{array}$ & $\begin{array}{l}8.8 \\
8.7 \\
8.8 \\
8.8 \\
8.8 \\
8.8 \\
8.7\end{array}$ & $\begin{array}{l}97 \\
96 \\
97 \\
96 \\
96 \\
96 \\
96\end{array}$ \\
\hline
\end{tabular}


Table 8. Water-quality data for station 402619080362201, Ohio River at river mile 61.0, main channel, June to October, 1995.

[ft $=$ feet; $\mu \mathrm{s} / \mathrm{cm}=$ microsiemens per centimeter; ${ }^{\circ} \mathrm{C}=$ degrees $\mathrm{Celsius;}$ $\mathrm{mg} / \mathrm{L}=$ mililigrams per liter; - = data not collected]

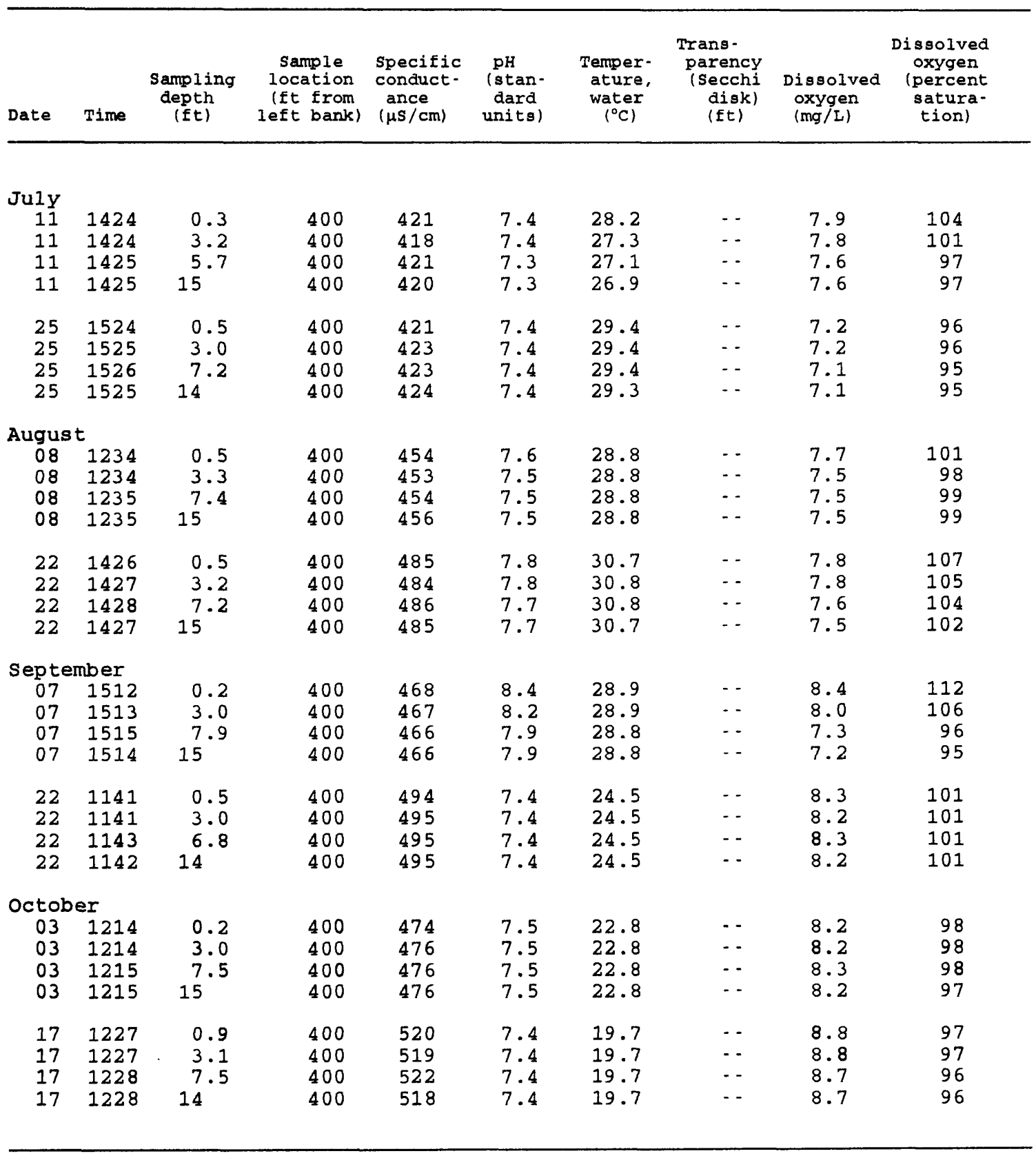


Table 9. Water-quality data for station 402620080364201, Ohio River at river mile 61.0, back channel, June to October, 1995.

[ft $=$ feet; $\mu \mathrm{S} / \mathrm{cm}=$ microsiemens per centimeter; ${ }^{\circ} \mathrm{C}=$ degrees Celsius; $\mathrm{mg} / \mathrm{L}=\mathrm{mili}$ igrams per liter; $-{ }^{-}$= data not collected]

\begin{tabular}{|c|c|c|c|c|c|c|c|c|c|}
\hline Date & Time & $\begin{array}{l}\text { Sampling } \\
\text { depth } \\
\text { (ft) }\end{array}$ & $\begin{array}{c}\text { Sample } \\
\text { location } \\
\text { (ft from } \\
\text { left bank) }\end{array}$ & $\begin{array}{l}\text { Specific } \\
\text { conduct- } \\
\text { ance } \\
(\mu \mathrm{S} / \mathrm{cm})\end{array}$ & $\begin{array}{l}\mathrm{pH} \\
\text { (stan- } \\
\text { dard } \\
\text { units) }\end{array}$ & $\begin{array}{c}\text { Temper- } \\
\text { ature, } \\
\text { water } \\
\left({ }^{\circ} \mathrm{C}\right)\end{array}$ & $\begin{array}{l}\text { Trans- } \\
\text { parency } \\
\text { (Secchi } \\
\text { disk) } \\
\text { (ft) }\end{array}$ & $\begin{array}{l}\text { Dissolved } \\
\text { oxygen } \\
(\mathrm{mg} / \mathrm{L})\end{array}$ & $\begin{array}{c}\text { Dissolved } \\
\text { oxygen } \\
\text { (percent } \\
\text { satura- } \\
\text { tion) }\end{array}$ \\
\hline $\begin{array}{r}\text { July } \\
11 \\
11 \\
11 \\
11\end{array}$ & $\begin{array}{l}1428 \\
1428 \\
1429 \\
1429\end{array}$ & $\begin{array}{l}0.3 \\
3.2 \\
4.0 \\
8.9\end{array}$ & $\begin{array}{l}2,000 \\
2,000 \\
2,000 \\
2,000\end{array}$ & $\begin{array}{l}419 \\
419 \\
420 \\
420\end{array}$ & $\begin{array}{l}7.3 \\
7.3 \\
7.3 \\
7.3\end{array}$ & $\begin{array}{l}27.1 \\
27.1 \\
27.1 \\
27.1\end{array}$ & $\begin{array}{l}-- \\
\cdots \\
\cdots\end{array}$ & $\begin{array}{l}7.8 \\
7.8 \\
7.8 \\
7.8\end{array}$ & $\begin{array}{l}100 \\
100 \\
100 \\
100\end{array}$ \\
\hline $\begin{array}{l}25 \\
25 \\
25 \\
25\end{array}$ & $\begin{array}{l}1535 \\
1535 \\
1537 \\
1536\end{array}$ & $\begin{array}{r}0.6 \\
3.0 \\
5.6 \\
11\end{array}$ & $\begin{array}{l}2,000 \\
2,000 \\
2,000 \\
2,000\end{array}$ & $\begin{array}{l}421 \\
421 \\
421 \\
423\end{array}$ & $\begin{array}{l}7.4 \\
7.4 \\
7.4 \\
7.4\end{array}$ & $\begin{array}{l}29.4 \\
29.4 \\
29.3 \\
29.3\end{array}$ & $\begin{array}{l}\cdots \\
\cdots \\
--\end{array}$ & $\begin{array}{l}7.1 \\
7.1 \\
7.1 \\
7.0\end{array}$ & $\begin{array}{l}95 \\
94 \\
95 \\
94\end{array}$ \\
\hline $\begin{array}{c}\text { Augus } \\
08 \\
08 \\
08 \\
08\end{array}$ & $\begin{array}{l}\text { t } \\
1228 \\
1228 \\
1230 \\
1229\end{array}$ & $\begin{array}{l}0.8 \\
3.1 \\
4.3 \\
7.5\end{array}$ & $\begin{array}{l}2,000 \\
2,000 \\
2,000 \\
2,000\end{array}$ & $\begin{array}{l}457 \\
456 \\
456 \\
453\end{array}$ & $\begin{array}{l}7.6 \\
7.5 \\
7.5 \\
7.5\end{array}$ & $\begin{array}{l}29.0 \\
29.0 \\
29.0 \\
29.0\end{array}$ & $\begin{array}{l}\cdots \\
\cdots \\
\cdots\end{array}$ & $\begin{array}{l}7.6 \\
7.4 \\
7.3 \\
7.5\end{array}$ & $\begin{array}{r}100 \\
97 \\
97 \\
99\end{array}$ \\
\hline $\begin{array}{l}22 \\
22 \\
22 \\
22\end{array}$ & $\begin{array}{l}1430 \\
1431 \\
1432 \\
1431\end{array}$ & $\begin{array}{r}0.3 \\
2.9 \\
4.6 \\
10\end{array}$ & $\begin{array}{l}2,000 \\
2,000 \\
2,000 \\
2,000\end{array}$ & $\begin{array}{l}483 \\
485 \\
484 \\
483\end{array}$ & $\begin{array}{l}7.8 \\
7.8 \\
7.8 \\
7.7\end{array}$ & $\begin{array}{l}30.8 \\
30.8 \\
30.8 \\
30.8\end{array}$ & $\begin{array}{l}-- \\
- \\
-- \\
--\end{array}$ & $\begin{array}{l}7.7 \\
7.9 \\
7.6 \\
7.7\end{array}$ & $\begin{array}{l}105 \\
107 \\
103 \\
105\end{array}$ \\
\hline $\begin{array}{c}\text { Septe } \\
07 \\
07 \\
07 \\
07\end{array}$ & $\begin{array}{l}\text { mber } \\
1518 \\
1519 \\
1521 \\
1520\end{array}$ & $\begin{array}{l}0.2 \\
3.0 \\
4.5 \\
9.1\end{array}$ & $\begin{array}{l}2,000 \\
2,000 \\
2,000 \\
2,000\end{array}$ & $\begin{array}{l}468 \\
468 \\
469 \\
470\end{array}$ & $\begin{array}{l}8.1 \\
8.1 \\
8.1 \\
8.1\end{array}$ & $\begin{array}{l}28.8 \\
28.9 \\
28.8 \\
28.8\end{array}$ & $\begin{array}{l}-- \\
-- \\
--\end{array}$ & $\begin{array}{l}7.9 \\
7.8 \\
7.8 \\
7.7\end{array}$ & $\begin{array}{l}104 \\
104 \\
104 \\
102\end{array}$ \\
\hline $\begin{array}{l}22 \\
22 \\
22 \\
22\end{array}$ & $\begin{array}{l}1136 \\
1136 \\
1138 \\
1137\end{array}$ & $\begin{array}{l}0.9 \\
3.1 \\
3.4 \\
7.0\end{array}$ & $\begin{array}{l}2,000 \\
2,000 \\
2,000 \\
2,000\end{array}$ & $\begin{array}{l}501 \\
496 \\
501 \\
499\end{array}$ & $\begin{array}{l}7.4 \\
7.4 \\
7.4 \\
7.4\end{array}$ & $\begin{array}{l}24.5 \\
24.5 \\
24.5 \\
24.5\end{array}$ & $\begin{array}{l}-- \\
- \\
--\end{array}$ & $\begin{array}{l}8.2 \\
8.3 \\
8.2 \\
8.2\end{array}$ & $\begin{array}{l}100 \\
101 \\
100 \\
101\end{array}$ \\
\hline $\begin{array}{c}\text { Octob } \\
03 \\
03 \\
03 \\
03\end{array}$ & $\begin{array}{l}\text { er } \\
1209 \\
1210 \\
1211 \\
1210\end{array}$ & $\begin{array}{r}0.2 \\
2.9 \\
6.1 \\
12\end{array}$ & $\begin{array}{l}2,000 \\
2,000 \\
2,000 \\
2,000\end{array}$ & $\begin{array}{l}475 \\
476 \\
476 \\
477\end{array}$ & $\begin{array}{l}7.5 \\
7.5 \\
7.5 \\
7.5\end{array}$ & $\begin{array}{l}22.8 \\
22.8 \\
22.8 \\
22.8\end{array}$ & $\begin{array}{l}-- \\
-- \\
--\end{array}$ & $\begin{array}{l}8.3 \\
8.2 \\
8.3 \\
8.3\end{array}$ & $\begin{array}{l}99 \\
97 \\
99 \\
98\end{array}$ \\
\hline $\begin{array}{l}17 \\
17 \\
17 \\
17\end{array}$ & $\begin{array}{l}1222 \\
1223 \\
1224 \\
1223\end{array}$ & $\begin{array}{r}0.9 \\
3.1 \\
5.6 \\
12\end{array}$ & $\begin{array}{l}2,000 \\
2,000 \\
2,000 \\
2,000\end{array}$ & $\begin{array}{l}520 \\
519 \\
520 \\
519\end{array}$ & $\begin{array}{l}7.4 \\
7.4 \\
7.4 \\
7.4\end{array}$ & $\begin{array}{l}19.6 \\
19.7 \\
19.6 \\
19.6\end{array}$ & $\begin{array}{l}-- \\
\cdots \\
-\end{array}$ & $\begin{array}{l}8.8 \\
8.7 \\
8.7 \\
8.7\end{array}$ & $\begin{array}{l}97 \\
96 \\
96 \\
96\end{array}$ \\
\hline
\end{tabular}


Table 10. Water-quality data for station 402426080362901, Ohio River at river mile 63.2, main channel, June to October, 1995.

Ift $=$ feet $; \mu \mathrm{S} / \mathrm{cm}=$ microsiemens per centimeter; ${ }^{\circ} \mathrm{C}=$ degrees Celsius;

$\mathrm{mg} / \mathrm{L}=$ milligrams per liter; $\cdot$ - = data not collected]

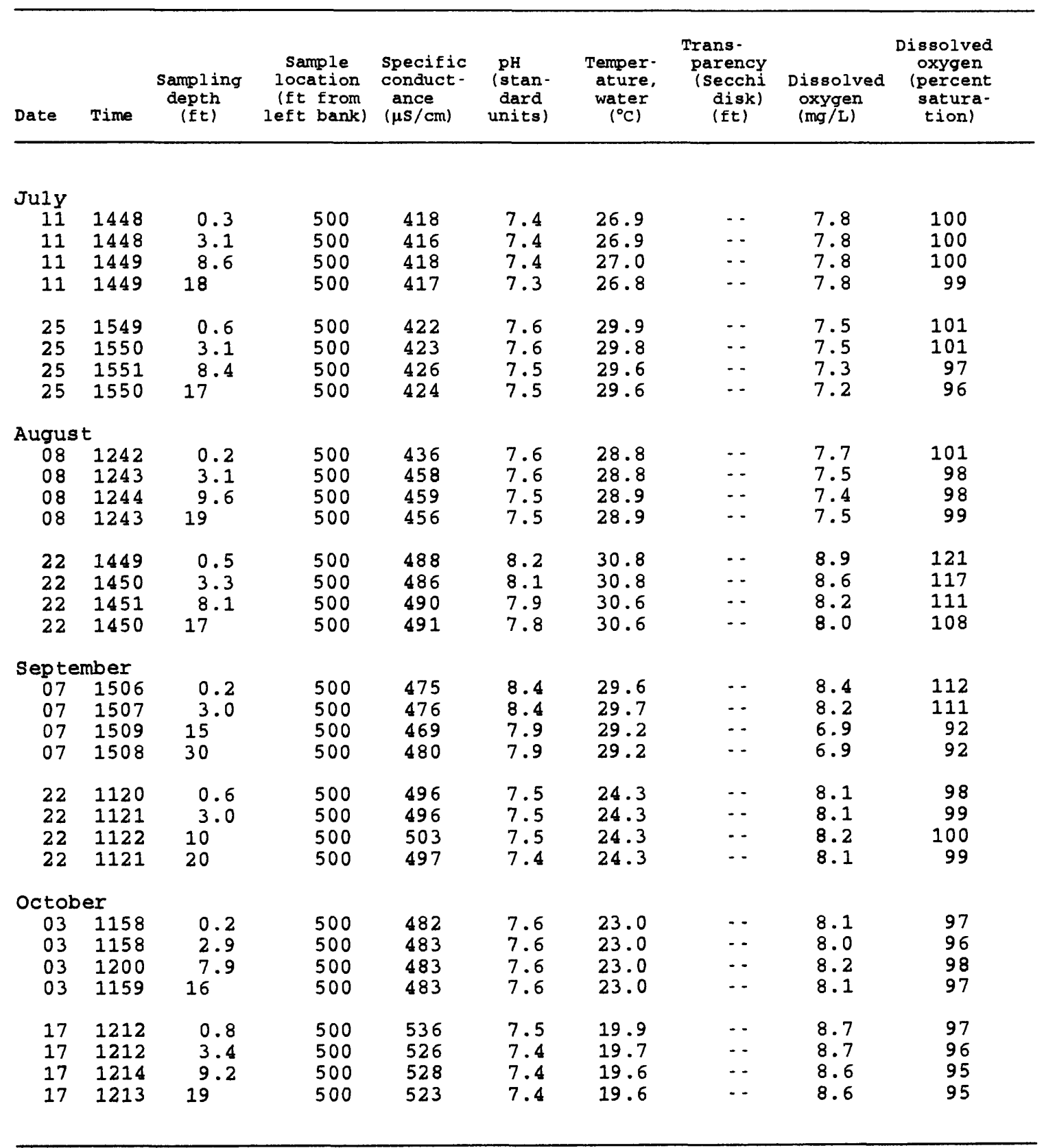


Table 11. Water-quality data for station 402428080364601, Ohio River at river mile 63.2, back channel, June to October, 1995.

lft $=$ feet $; \mu \mathrm{s} / \mathrm{cm}=$ microsiemens per centimeter $;{ }^{\circ} \mathrm{C}=$ degrees Celsius;

$\mathrm{mg} / \mathrm{L}=\mathrm{mili}$ igrams per liter; $\cdot-$ = data not collected]

\begin{tabular}{|c|c|c|c|c|c|c|c|c|c|}
\hline Date & Time & $\begin{array}{l}\text { Sampling } \\
\text { depth } \\
\text { (ft) }\end{array}$ & $\begin{array}{c}\text { sample } \\
\text { location } \\
\text { (ft from } \\
\text { left bank) }\end{array}$ & $\begin{array}{l}\text { Specific } \\
\text { conduct- } \\
\text { ance } \\
(\mu \mathrm{s} / \mathrm{cm})\end{array}$ & $\begin{array}{l}\mathrm{pH} \\
\text { (stan- } \\
\text { dard } \\
\text { units) }\end{array}$ & $\begin{array}{c}\text { Temper- } \\
\text { ature, } \\
\text { water } \\
\left({ }^{\circ} \mathrm{C}\right)\end{array}$ & $\begin{array}{l}\text { Trans- } \\
\text { parency } \\
\text { (Secchi } \\
\text { disk) } \\
\text { (ft) }\end{array}$ & $\begin{array}{l}\text { Dissolved } \\
\text { oxygen } \\
(\mathrm{mg} / \mathrm{L})\end{array}$ & $\begin{array}{c}\text { Dissolved } \\
\text { oxygen } \\
\text { (percent } \\
\text { satura- } \\
\text { tion) }\end{array}$ \\
\hline $\begin{array}{r}\text { July } \\
11 \\
11 \\
11 \\
11\end{array}$ & $\begin{array}{l}1434 \\
1435 \\
1436 \\
1435\end{array}$ & $\begin{array}{l}0.3 \\
2.9 \\
12 \\
23\end{array}$ & $\begin{array}{l}1,800 \\
1,800 \\
1,800 \\
1,800\end{array}$ & $\begin{array}{l}417 \\
417 \\
414 \\
417\end{array}$ & $\begin{array}{l}7.5 \\
7.5 \\
7.3 \\
7.3\end{array}$ & $\begin{array}{l}27.3 \\
27.3 \\
25.9 \\
25.9\end{array}$ & $\begin{array}{l}\cdots \\
-- \\
\cdots \\
-\end{array}$ & $\begin{array}{l}8.3 \\
8.4 \\
7.6 \\
7.5\end{array}$ & $\begin{array}{r}106 \\
108 \\
96 \\
94\end{array}$ \\
\hline $\begin{array}{l}25 \\
25 \\
25 \\
25\end{array}$ & $\begin{array}{l}1544 \\
1544 \\
1546 \\
1546\end{array}$ & $\begin{array}{l}0.4 \\
2.9 \\
13 \\
26\end{array}$ & $\begin{array}{l}1,800 \\
1,800 \\
1,800 \\
1,800\end{array}$ & $\begin{array}{l}421 \\
421 \\
421 \\
418\end{array}$ & $\begin{array}{l}7.6 \\
7.6 \\
7.4 \\
7.4\end{array}$ & $\begin{array}{l}29.7 \\
29.7 \\
28.8 \\
28.7\end{array}$ & $\begin{array}{l}-- \\
\cdots \\
\cdots\end{array}$ & $\begin{array}{l}7.9 \\
7.9 \\
6.8 \\
6.6\end{array}$ & $\begin{array}{r}107 \\
106 \\
89 \\
87\end{array}$ \\
\hline $\begin{array}{c}\text { Augus } \\
08 \\
08 \\
08 \\
08\end{array}$ & $\begin{array}{l}1249 \\
1249 \\
1250 \\
1250\end{array}$ & $\begin{array}{l}0.8 \\
3.2 \\
14 \\
28\end{array}$ & $\begin{array}{l}1,800 \\
1,800 \\
1,800 \\
1,800\end{array}$ & $\begin{array}{l}461 \\
461 \\
462 \\
463\end{array}$ & $\begin{array}{l}7.6 \\
7.5 \\
7.5 \\
7.5\end{array}$ & $\begin{array}{l}28.9 \\
28.9 \\
28.9 \\
28.9\end{array}$ & $\begin{array}{l}-- \\
-- \\
--\end{array}$ & $\begin{array}{l}7.4 \\
7.3 \\
7.3 \\
7.3\end{array}$ & $\begin{array}{l}97 \\
97 \\
96 \\
96\end{array}$ \\
\hline $\begin{array}{l}22 \\
22 \\
22 \\
22\end{array}$ & $\begin{array}{l}1446 \\
1446 \\
1447 \\
1447\end{array}$ & $\begin{array}{l}0.5 \\
3.3 \\
12 \\
26\end{array}$ & $\begin{array}{l}1,800 \\
1,800 \\
1,800 \\
1,800\end{array}$ & $\begin{array}{l}486 \\
486 \\
487 \\
487\end{array}$ & $\begin{array}{l}8.2 \\
8.2 \\
8.0 \\
7.8\end{array}$ & $\begin{array}{l}30.1 \\
30.1 \\
29.9 \\
29.7\end{array}$ & $\begin{array}{l}-- \\
-- \\
--\end{array}$ & $\begin{array}{l}9.2 \\
9.2 \\
8.4 \\
7.8\end{array}$ & $\begin{array}{l}124 \\
124 \\
113 \\
104\end{array}$ \\
\hline $\begin{array}{c}\text { Septe } \\
07 \\
07 \\
07 \\
07\end{array}$ & $\begin{array}{l}\text { nber } \\
1500 \\
1501 \\
1503 \\
1502\end{array}$ & $\begin{array}{r}0.2 \\
3.0 \\
7.2 \\
13\end{array}$ & $\begin{array}{l}1,800 \\
1,800 \\
1,800 \\
1,800\end{array}$ & $\begin{array}{l}469 \\
466 \\
469 \\
471\end{array}$ & $\begin{array}{l}8.6 \\
8.5 \\
8.3 \\
8.2\end{array}$ & $\begin{array}{l}28.8 \\
28.6 \\
28.4 \\
28.4\end{array}$ & $\begin{array}{l}\cdots \\
\cdots \\
-\end{array}$ & $\begin{array}{l}9.2 \\
8.9 \\
8.1 \\
8.0\end{array}$ & $\begin{array}{l}122 \\
118 \\
107 \\
106\end{array}$ \\
\hline $\begin{array}{l}22 \\
22 \\
22 \\
22\end{array}$ & $\begin{array}{l}1126 \\
1126 \\
1130 \\
1128\end{array}$ & $\begin{array}{l}1.1 \\
3.0 \\
16 \\
34\end{array}$ & $\begin{array}{l}1,800 \\
1,800 \\
1,800 \\
1,800\end{array}$ & $\begin{array}{l}499 \\
496 \\
505 \\
503\end{array}$ & $\begin{array}{l}7.4 \\
7.4 \\
7.4 \\
7.4\end{array}$ & $\begin{array}{l}24.0 \\
24.0 \\
24.0 \\
24.0\end{array}$ & $\begin{array}{l}-- \\
-- \\
-\end{array}$ & $\begin{array}{l}7.9 \\
7.9 \\
7.9 \\
7.9\end{array}$ & $\begin{array}{l}96 \\
96 \\
96 \\
95\end{array}$ \\
\hline $\begin{array}{c}\text { Octob } \\
03 \\
03 \\
03 \\
03\end{array}$ & $\begin{array}{l}\text { er } \\
1202 \\
1203 \\
1204 \\
1203\end{array}$ & $\begin{array}{c}0.2 \\
2.9 \\
14 \\
28\end{array}$ & $\begin{array}{l}1,800 \\
1,800 \\
1,800 \\
1,800\end{array}$ & $\begin{array}{l}477 \\
479 \\
480 \\
480\end{array}$ & $\begin{array}{l}7.5 \\
7.5 \\
7.5 \\
7.5\end{array}$ & $\begin{array}{l}22.3 \\
22.2 \\
22.2 \\
22.2\end{array}$ & $\begin{array}{l}- \\
\cdots \\
- \\
-\end{array}$ & $\begin{array}{l}8.2 \\
8.0 \\
8.1 \\
7.9\end{array}$ & $\begin{array}{l}97 \\
94 \\
95 \\
93\end{array}$ \\
\hline $\begin{array}{l}17 \\
17 \\
17 \\
17\end{array}$ & $\begin{array}{l}1216 \\
1216 \\
1217 \\
1217\end{array}$ & $\begin{array}{l}0.8 \\
3.4 \\
15 \\
29\end{array}$ & $\begin{array}{l}1,800 \\
1,800 \\
1,800 \\
1,800\end{array}$ & $\begin{array}{l}522 \\
522 \\
520 \\
525\end{array}$ & $\begin{array}{l}7.4 \\
7.4 \\
7.4 \\
7.4\end{array}$ & $\begin{array}{l}19.0 \\
18.9 \\
18.8 \\
18.8\end{array}$ & $\begin{array}{l}\cdots \\
-- \\
--\end{array}$ & $\begin{array}{l}8.9 \\
8.6 \\
8.6 \\
8.6\end{array}$ & $\begin{array}{l}96 \\
94 \\
93 \\
93\end{array}$ \\
\hline
\end{tabular}


Table 12. Water-quality data for station 402329080375901, Ohio River at river mile 65.0, June to october, 1995.

[ft $=$ feet; $\mu \mathrm{S} / \mathrm{cm}=$ microsiemens per centimeter; ${ }^{\circ} \mathrm{C}=$ degrees Celsius; $\mathrm{mg} / \mathrm{L}=$ mililigrams per liter; $\cdot-$ = data not collected]

\begin{tabular}{|c|c|c|c|c|c|c|c|c|c|}
\hline Date & Time & $\begin{array}{l}\text { Sampling } \\
\text { depth } \\
\text { (Et) }\end{array}$ & $\begin{array}{c}\text { Sample } \\
\text { location } \\
\text { (ft from } \\
\text { left bank) }\end{array}$ & $\begin{array}{l}\text { Specific } \\
\text { conduct- } \\
\text { ance } \\
(\mu \mathrm{s} / \mathrm{cm})\end{array}$ & $\begin{array}{l}\text { pH } \\
\text { (stan- } \\
\text { dard } \\
\text { units) }\end{array}$ & $\begin{array}{c}\text { Temper- } \\
\text { ature, } \\
\text { water } \\
\left({ }^{\circ} \mathrm{C}\right)\end{array}$ & $\begin{array}{l}\text { Trans- } \\
\text { parency } \\
\text { (Secchi } \\
\text { disk) } \\
(\mathrm{Et})\end{array}$ & $\begin{array}{l}\text { Dissolved } \\
\text { oxygen } \\
(\mathrm{mg} / \mathrm{L})\end{array}$ & $\begin{array}{c}\text { Dissolved } \\
\text { oxygen } \\
\text { (percent } \\
\text { satura- } \\
\text { tion) }\end{array}$ \\
\hline $\begin{array}{r}\text { July } \\
11 \\
11 \\
11 \\
11\end{array}$ & $\begin{array}{l}1454 \\
1454 \\
1455 \\
1455\end{array}$ & $\begin{array}{l}0.3 \\
3.2 \\
20 \\
39\end{array}$ & $\begin{array}{l}600 \\
600 \\
600 \\
600\end{array}$ & $\begin{array}{l}413 \\
415 \\
413 \\
413\end{array}$ & $\begin{array}{l}7.5 \\
7.5 \\
7.3 \\
7.3\end{array}$ & $\begin{array}{l}27.2 \\
27.2 \\
26.2 \\
26.2\end{array}$ & $\begin{array}{l}-- \\
-- \\
--\end{array}$ & $\begin{array}{l}8.1 \\
8.1 \\
7.7 \\
7.7\end{array}$ & $\begin{array}{r}104 \\
105 \\
97 \\
97\end{array}$ \\
\hline $\begin{array}{l}25 \\
25 \\
25 \\
25\end{array}$ & $\begin{array}{l}1555 \\
1555 \\
1556 \\
1556\end{array}$ & $\begin{array}{l}0.7 \\
3.1 \\
20 \\
41\end{array}$ & $\begin{array}{l}600 \\
600 \\
600 \\
600\end{array}$ & $\begin{array}{l}425 \\
424 \\
422 \\
419\end{array}$ & $\begin{array}{l}7.6 \\
7.6 \\
7.5 \\
7.5\end{array}$ & $\begin{array}{l}30.0 \\
29.7 \\
29.1 \\
29.1\end{array}$ & $\begin{array}{l}-- \\
\cdots \\
--\end{array}$ & $\begin{array}{l}7.7 \\
7.4 \\
6.9 \\
6.8\end{array}$ & $\begin{array}{r}103 \\
99 \\
92 \\
91\end{array}$ \\
\hline $\begin{array}{c}\text { Augus } \\
08 \\
08 \\
08 \\
08\end{array}$ & $\begin{array}{l}\text { t } \\
1317 \\
1317 \\
1319 \\
1318\end{array}$ & $\begin{array}{l}0.3 \\
3.0 \\
19 \\
38\end{array}$ & $\begin{array}{l}600 \\
600 \\
600 \\
600\end{array}$ & $\begin{array}{l}462 \\
464 \\
467 \\
460\end{array}$ & $\begin{array}{l}7.6 \\
7.6 \\
7.5 \\
7.5\end{array}$ & $\begin{array}{l}28.9 \\
28.9 \\
28.9 \\
28.8\end{array}$ & $\begin{array}{l}-- \\
-- \\
-\end{array}$ & $\begin{array}{l}7.4 \\
7.5 \\
7.4 \\
7.2\end{array}$ & $\begin{array}{l}98 \\
98 \\
97 \\
95\end{array}$ \\
\hline $\begin{array}{l}22 \\
22 \\
22 \\
22\end{array}$ & $\begin{array}{l}1454 \\
1455 \\
1457 \\
1456\end{array}$ & $\begin{array}{l}0.4 \\
3.4 \\
20 \\
43\end{array}$ & $\begin{array}{l}600 \\
600 \\
600 \\
600\end{array}$ & $\begin{array}{l}489 \\
490 \\
491 \\
492\end{array}$ & $\begin{array}{l}8.3 \\
8.2 \\
7.9 \\
7.9\end{array}$ & $\begin{array}{l}30.7 \\
30.7 \\
30.6 \\
30.5\end{array}$ & $\begin{array}{l}- \\
\cdots \\
- \\
-\end{array}$ & $\begin{array}{l}9.1 \\
8.8 \\
8.0 \\
8.1\end{array}$ & $\begin{array}{l}124 \\
120 \\
109 \\
110\end{array}$ \\
\hline $\begin{array}{c}\text { Septe } \\
07 \\
07 \\
07 \\
07\end{array}$ & $\begin{array}{l}\text { mber } \\
1451 \\
1452 \\
1454 \\
1453\end{array}$ & $\begin{array}{l}0.2 \\
3.0 \\
15 \\
30\end{array}$ & $\begin{array}{l}600 \\
600 \\
600 \\
600\end{array}$ & $\begin{array}{l}471 \\
472 \\
474 \\
467\end{array}$ & $\begin{array}{l}8.5 \\
8.3 \\
8.1 \\
7.9\end{array}$ & $\begin{array}{l}28.9 \\
28.8 \\
28.8 \\
28.7\end{array}$ & $\begin{array}{l}-- \\
- \\
-\end{array}$ & $\begin{array}{l}8.9 \\
8.5 \\
7.7 \\
7.4\end{array}$ & $\begin{array}{r}118 \\
114 \\
102 \\
99\end{array}$ \\
\hline $\begin{array}{l}22 \\
22 \\
22 \\
22\end{array}$ & $\begin{array}{l}1113 \\
1114 \\
1115 \\
1114\end{array}$ & $\begin{array}{l}0.7 \\
3.0 \\
21 \\
41\end{array}$ & $\begin{array}{l}600 \\
600 \\
600 \\
600\end{array}$ & $\begin{array}{l}503 \\
507 \\
508 \\
513\end{array}$ & $\begin{array}{l}7.4 \\
7.4 \\
7.4 \\
7.4\end{array}$ & $\begin{array}{l}24.4 \\
24.4 \\
24.4 \\
24.3\end{array}$ & $\begin{array}{l}\cdots \\
\cdots \\
-\end{array}$ & $\begin{array}{l}7.9 \\
7.9 \\
7.9 \\
7.8\end{array}$ & $\begin{array}{l}97 \\
96 \\
96 \\
96\end{array}$ \\
\hline $\begin{array}{c}\text { Octor } \\
03 \\
03 \\
03 \\
03\end{array}$ & $\begin{array}{l}\text { er } \\
1150 \\
1150 \\
1152 \\
1151\end{array}$ & $\begin{array}{l}1.0 \\
2.7 \\
19 \\
37\end{array}$ & $\begin{array}{l}600 \\
600 \\
600 \\
600\end{array}$ & $\begin{array}{l}488 \\
488 \\
488 \\
485\end{array}$ & $\begin{array}{l}7.5 \\
7.5 \\
7.5 \\
7.5\end{array}$ & $\begin{array}{l}22.8 \\
22.8 \\
22.8 \\
22.8\end{array}$ & $\begin{array}{l}\cdots \\
\cdots \\
-\end{array}$ & $\begin{array}{l}7.9 \\
7.9 \\
8.0 \\
7.9\end{array}$ & $\begin{array}{l}94 \\
94 \\
95 \\
94\end{array}$ \\
\hline $\begin{array}{l}17 \\
17 \\
17 \\
17\end{array}$ & $\begin{array}{l}1206 \\
1206 \\
1208 \\
1207\end{array}$ & $\begin{array}{l}0.6 \\
3.5 \\
20 \\
39\end{array}$ & $\begin{array}{l}600 \\
600 \\
600 \\
600\end{array}$ & $\begin{array}{l}524 \\
523 \\
521 \\
520\end{array}$ & $\begin{array}{l}7.4 \\
7.4 \\
7.4 \\
7.4\end{array}$ & $\begin{array}{l}19.3 \\
19.1 \\
18.9 \\
18.9\end{array}$ & $\begin{array}{l}-- \\
-- \\
--\end{array}$ & $\begin{array}{l}8.7 \\
8.6 \\
8.6 \\
8.5\end{array}$ & $\begin{array}{l}95 \\
94 \\
93 \\
92\end{array}$ \\
\hline
\end{tabular}


Table 13. Water-quality data for station 402246080364601, Ohio River at river mile 66.4, June to October, 1995.

lft = feet; $\mu \mathrm{s} / \mathrm{cm}=$ microsiemens per centimeter; ${ }^{\circ} \mathrm{C}=$ degrees Celsius; $\mathrm{mg} / \mathrm{L}=$ milligrams per liter; -. = data not collected]

\begin{tabular}{|c|c|c|c|c|c|c|c|c|c|}
\hline Date & Time & $\begin{array}{l}\text { Sampling } \\
\text { depth } \\
\text { (ft) }\end{array}$ & $\begin{array}{c}\text { Sample } \\
\text { location } \\
\text { (ft from } \\
\text { left bank) }\end{array}$ & $\begin{array}{l}\text { Specific } \\
\text { conduct- } \\
\text { ance } \\
(\mu \mathrm{s} / \mathrm{cm})\end{array}$ & $\begin{array}{l}\text { pH } \\
\text { (stan- } \\
\text { dard } \\
\text { units) }\end{array}$ & $\begin{array}{c}\text { Temper- } \\
\text { ature, } \\
\text { water } \\
\left({ }^{\circ} \mathrm{C}\right)\end{array}$ & $\begin{array}{l}\text { Trans- } \\
\text { parency } \\
\text { (Secchi } \\
\text { disk) } \\
\text { (ft) }\end{array}$ & $\begin{array}{l}\text { Dissolved } \\
\text { oxygen } \\
\text { (mg/L) }\end{array}$ & $\begin{array}{c}\text { Dissolved } \\
\text { oxygen } \\
\text { (percent } \\
\text { satura- } \\
\text { tion) }\end{array}$ \\
\hline
\end{tabular}

$\begin{array}{rr}\text { July } & \\ 11 & 1501 \\ 11 & 1501 \\ 11 & 1502 \\ 11 & 1502 \\ 11 & 1503 \\ 11 & 1503 \\ 11 & 1504 \\ 11 & 1504 \\ 25 & 1601 \\ 25 & 1601 \\ 25 & 1602 \\ 25 & 1602 \\ 25 & 1603 \\ 25 & 1603 \\ 25 & 1604 \\ 25 & 1604\end{array}$

$\begin{array}{rll}0.3 & 600 & 411 \\ 3.0 & 600 & 411 \\ 5.1 & 600 & 412 \\ 9.8 & 600 & 413 \\ 15 & 600 & 414 \\ 20 & 600 & 411 \\ 25 & 600 & 414 \\ 29 & 600 & 411 \\ & & \\ 0.3 & 600 & 404 \\ 2.6 & 600 & 420 \\ 5.1 & 600 & 420 \\ 10 & 600 & 420 \\ 15 & 600 & 421 \\ 20 & 600 & 421 \\ 25 & 600 & 421 \\ 29 & 600 & 421\end{array}$

7.4
7.4
7.4
7.4
7.4
7.4
7.4
7.3
7.5
7.5
7.5
7.5
7.5
7.5
7.5
7.5

$26 \cdot 4$
$26 \cdot 4$
$26 \cdot 4$
$26 \cdot 4$
$26 \cdot 3$
$26 \cdot 3$
$26 \cdot 3$
$26 \cdot 3$
29.2
$29 \cdot 2$
29.2
29.2
29.1
29.1
29.1
29.1

$\begin{array}{lll}\ldots & 7.8 & 98 \\ \ldots & 7.8 & 99 \\ \ldots & 7.8 & 99 \\ \ldots & 7.8 & 98 \\ \ldots & 7.8 & 98 \\ \ldots & 7.8 & 98 \\ \ldots & 7.7 & 98 \\ \ldots & 7.7 & 97 \\ \ldots & 7.1 & 95 \\ \ldots & 7.1 & 95 \\ \ldots & 7.1 & 95 \\ -. & 7.1 & 94 \\ -. & 7.0 & 93 \\ -. & 7.0 & 93 \\ -. & 7.0 & 93 \\ .- & 7.0 & 93\end{array}$

August

$08 \quad 1324$

0.2
3.2
5.4
9.7
14
20
25
30

600

600

600

600

600

600

600

464

7.6

28.8

- 7.3

\section{6}

465

466

466

463

462

464

7.6
7.6

28.8

28.8

$\begin{array}{ll}7.5 & 28.8\end{array}$

$\begin{array}{ll}7.5 & 28.8 \\ 7.5 & 28.8\end{array}$

$\begin{array}{ll}7.5 & 28.8\end{array}$

$\begin{array}{ll}7.5 & 28.8 \\ 7.5 & 28.8\end{array}$

$\begin{array}{ll}- & 7.2 \\ - & 7.2\end{array}$

$\begin{array}{ll} & \\ \ldots & 7.2\end{array}$

$\begin{array}{ll}- & 7.2 \\ - & 7.3\end{array}$

- 7.2

. $\quad 7.2$

$460 \quad 7.5$

28.8

$600 \quad 492$

$22 \quad 1502$

$\begin{array}{ll}0.4 & 600 \\ 3.4 & 600\end{array}$

$5.6 \quad 600$

11

15

20

25

30

492
493

493

600

600

600

600

493

493

493

493

$8.0 \quad 30.6$

-. 7.3

$8.0 \quad 30.6$

$8.0 \quad 30.6$

$8.0 \quad 30.6$

$8.0 \quad 30.6$

$7.9 \quad 30.6$

$7.9 \quad 30.6$

$-$

\section{3}

- 8.3

. 8.2

. 8.2

. 8.1

. 8.1

\begin{tabular}{l}
. \\
\hline
\end{tabular}

30.6

- 7.9

$600 \quad 493$

\section{September}

$07 \quad 1442$

$07 \quad 1442$

$07 \quad 1443$

$07 \quad 1443$

0.2
3.0
5.0
10
15
20
25
30

$\begin{array}{ll}600 & 471 \\ 600 & 470 \\ 600 & 471 \\ 600 & 472 \\ 600 & 471 \\ 600 & 468 \\ 600 & 474 \\ 600 & 473\end{array}$

$\begin{array}{ll}8.2 & 28.6 \\ 8.2 & 28.6 \\ 8.2 & 28.6 \\ 8.1 & 28.6 \\ 8.1 & 28.5 \\ 8.0 & 28.5 \\ 8.0 & 28.5 \\ 8.0 & 28.4\end{array}$

$\begin{array}{ll}-. & 8.0 \\ \ldots & 8.0 \\ \ldots & 7.9 \\ -. & 7.8 \\ -. & 7.6 \\ -. & 7.5 \\ -. & 7.5 \\ - & 7.3\end{array}$

106

106

470
471

472

473

$\begin{array}{ll}8.0 & 28.5 \\ 8.0 & 28.4\end{array}$

7.3

103

100 
Table 13. Water-quality data for station 402246080364601, Ohio River at river mile 66.4, June to October, 1995, Continued.

LEt $=$ feet; $\mu \mathrm{S} / \mathrm{cm}=$ microsiemens per centimeter; ${ }^{\circ} \mathrm{C}=$ degrees Celsius;

$\mathrm{mg} / \mathrm{L}=$ milligrams per liter; - - = data not collected]

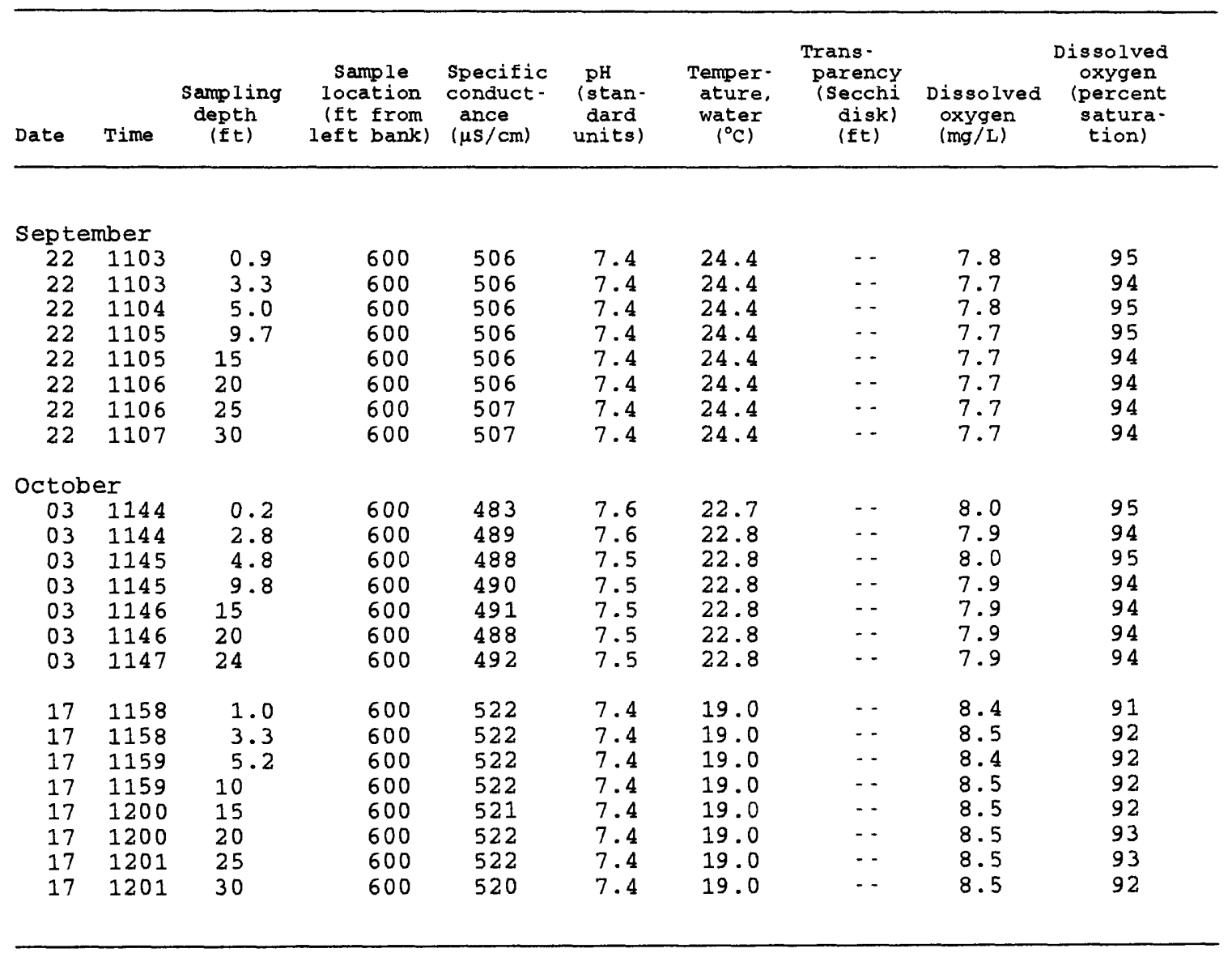


Table 14. Water-quality data for station 402213080362401 , Ohio River at river mile 67.1, June to October, 1995.

[ft $=$ feet $; \mu \mathrm{s} / \mathrm{cm}=$ microsiemens per centimeter; ${ }^{\circ} \mathrm{C}=$ degrees $\mathrm{Celsius}$; $\mathrm{mg} / \mathrm{L}=$ milligrams per liter; .. = data not collected]

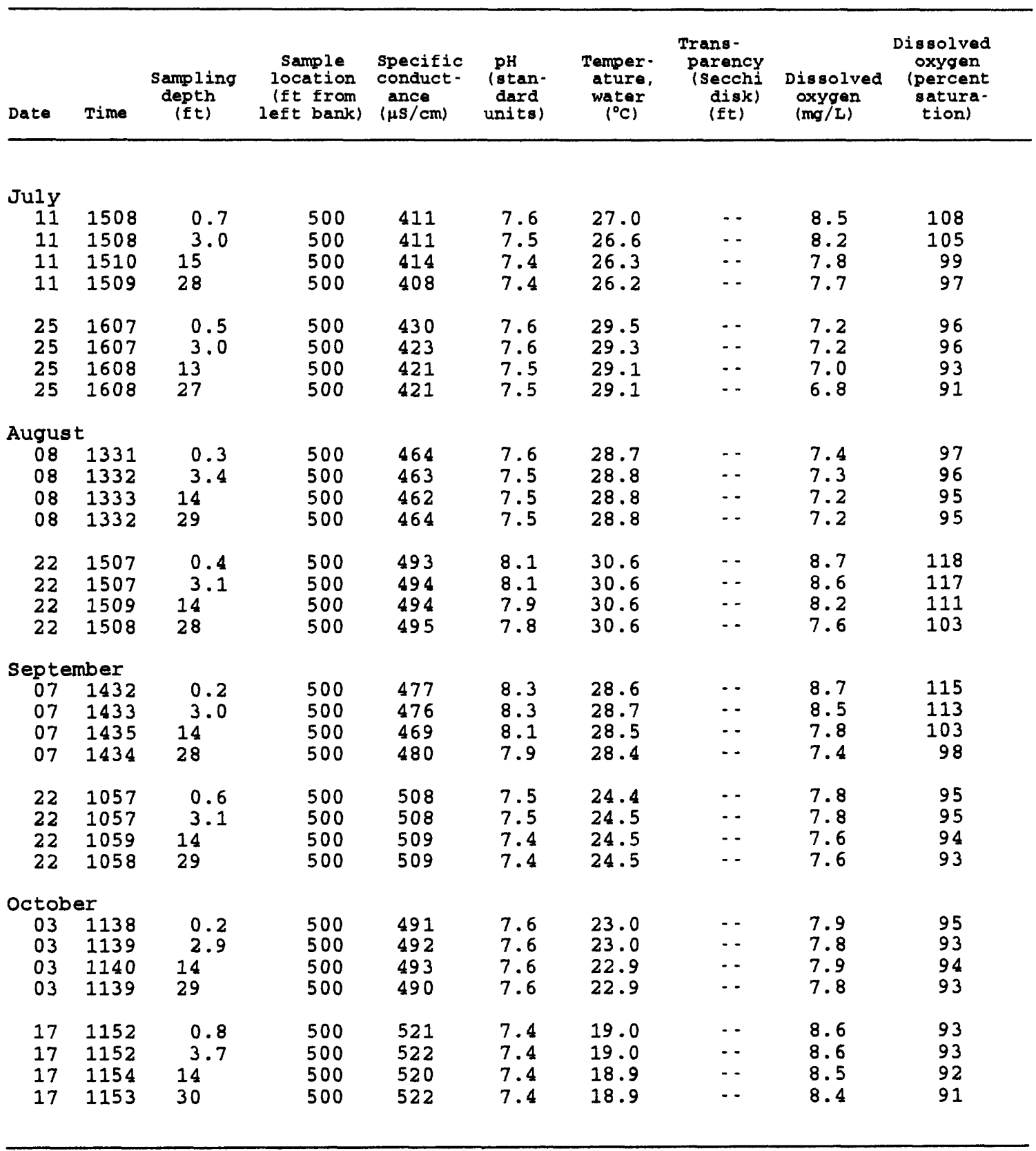


Table 15. Water-quality data for station 402051080363901, Ohio River at river mile 68.7, June to October, 1995.

ft $=$ feet $; \mu \mathrm{S} / \mathrm{cm}=$ microsiemens per centimeter $;{ }^{\circ} \mathrm{C}=$ degrees Celsius : $\mathrm{mg} / \mathrm{L}=$ milligrams per liter; -- = data not collected]

\begin{tabular}{|c|c|c|c|c|c|c|c|c|c|}
\hline Date & Time & $\begin{array}{l}\text { Sampling } \\
\text { depth } \\
\text { (ft) }\end{array}$ & $\begin{array}{c}\text { Sample } \\
\text { location } \\
\text { (ft from } \\
\text { left bank) }\end{array}$ & $\begin{array}{l}\text { Specific } \\
\text { conduct - } \\
\text { ance } \\
(\mu \mathrm{s} / \mathrm{cm})\end{array}$ & $\begin{array}{l}\text { pH } \\
\text { (stan- } \\
\text { dard } \\
\text { units) }\end{array}$ & $\begin{array}{c}\text { Temper- } \\
\text { ature, } \\
\text { water } \\
\left({ }^{\circ} \mathrm{C}\right)\end{array}$ & $\begin{array}{c}\text { Trans- } \\
\text { parency } \\
\text { (secchi } \\
\text { disk) } \\
\text { (ft) }\end{array}$ & $\begin{array}{l}\text { Dissolved } \\
\text { oxygen } \\
\text { (mg/L) }\end{array}$ & $\begin{array}{c}\text { Dissolved } \\
\text { oxygen } \\
\text { (percent } \\
\text { satura- } \\
\text { tion) }\end{array}$ \\
\hline
\end{tabular}

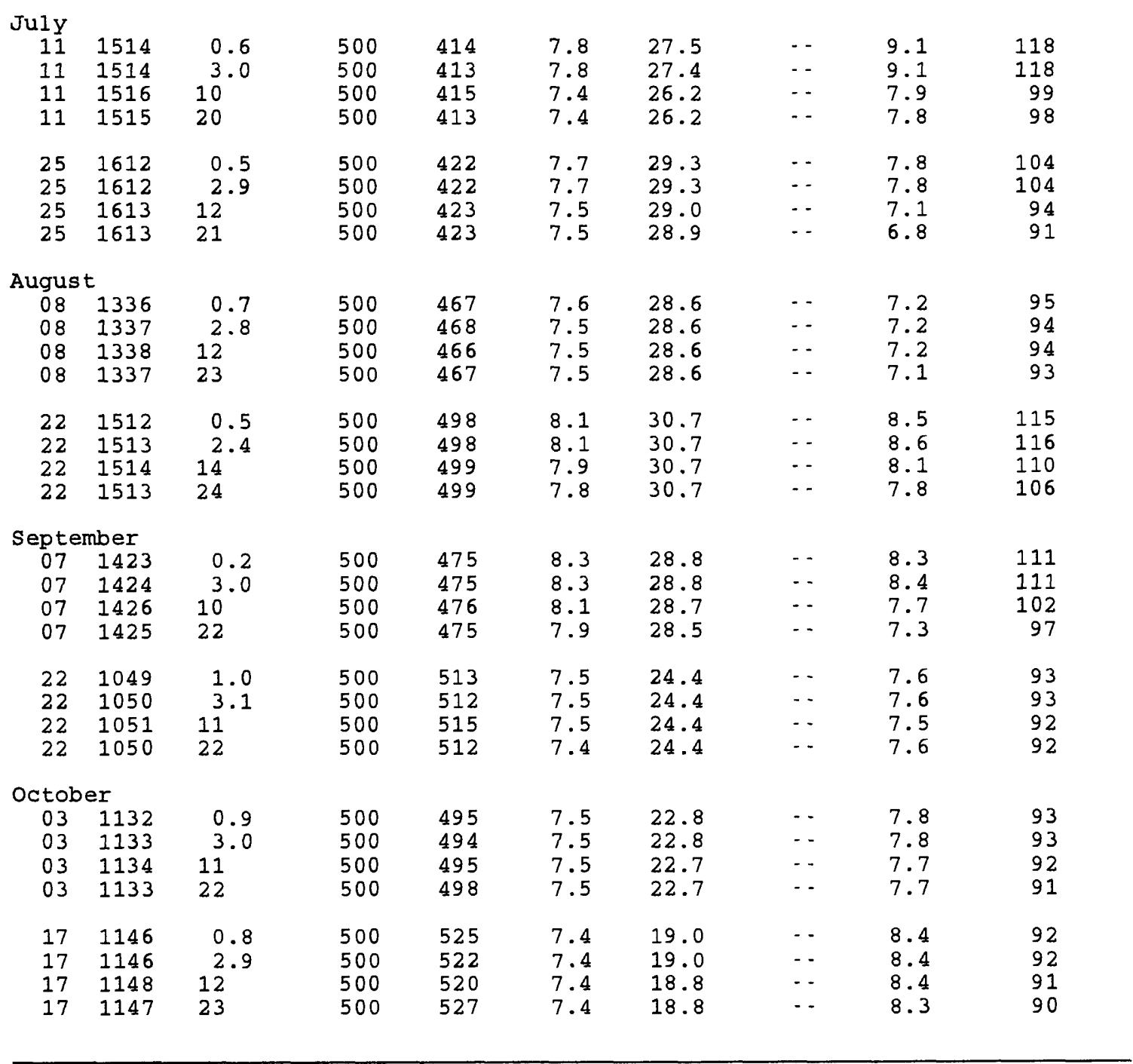


Table 16. Water-quality data for station 401838080360701, Ohio River at river mile 71.4, June to october, 1995.

[ft $=$ feet $; \mu \mathrm{s} / \mathrm{cm}=$ microsiemens per centimeter; ${ }^{\circ} \mathrm{C}=$ degrees Celsius ;

$\mathrm{mg} / \mathrm{L}=$ milligrams per 1 iter;..$=$ data not collected

\begin{tabular}{|c|c|c|c|c|c|c|c|c|c|}
\hline Date & Time & $\begin{array}{l}\text { Sampling } \\
\text { depth } \\
\text { (ft) }\end{array}$ & $\begin{array}{c}\text { Sample } \\
\text { location } \\
\text { (Et from } \\
\text { left bank) }\end{array}$ & $\begin{array}{l}\text { Specific } \\
\text { conduct- } \\
\text { ance } \\
(\mu s / c m)\end{array}$ & $\begin{array}{l}\text { pH } \\
\text { (stan- } \\
\text { dard } \\
\text { units) }\end{array}$ & $\begin{array}{c}\text { Temper- } \\
\text { ature, } \\
\text { water } \\
\left({ }^{\circ} \mathrm{C}\right)\end{array}$ & $\begin{array}{c}\text { Trans - } \\
\text { parency } \\
\text { (Secchi } \\
\text { disk) } \\
\text { (ft) }\end{array}$ & $\begin{array}{l}\text { Dissolved } \\
\text { oxygen } \\
\text { (mg/L) }\end{array}$ & $\begin{array}{c}\text { Dissolved } \\
\text { oxygen } \\
\text { (percent } \\
\text { satura- } \\
\text { tion) }\end{array}$ \\
\hline
\end{tabular}

\begin{tabular}{|c|c|c|c|c|c|c|c|c|c|}
\hline $\begin{array}{r}\text { July } \\
11 \\
11 \\
11 \\
11 \\
11 \\
11 \\
11 \\
11\end{array}$ & $\begin{array}{l}0559 \\
0559 \\
0600 \\
0600 \\
0601 \\
0602 \\
0602 \\
0603\end{array}$ & $\begin{array}{c}0.3 \\
3.0 \\
5.0 \\
10 \\
15 \\
20 \\
25 \\
29\end{array}$ & $\begin{array}{l}300 \\
300 \\
300 \\
300 \\
300 \\
300 \\
300 \\
300\end{array}$ & $\begin{array}{l}408 \\
409 \\
411 \\
410 \\
411 \\
410 \\
409 \\
412\end{array}$ & $\begin{array}{l}7.5 \\
7.5 \\
7.5 \\
7.5 \\
7.5 \\
7.5 \\
7.5 \\
7.5\end{array}$ & $\begin{array}{l}26.5 \\
26.5 \\
26.5 \\
26.5 \\
26.5 \\
26.5 \\
26.5 \\
26.5\end{array}$ & $\begin{array}{l}-- \\
-- \\
-- \\
-- \\
-- \\
-- \\
-- \\
-\end{array}$ & $\begin{array}{l}7.6 \\
7.6 \\
7.5 \\
7.6 \\
7.6 \\
7.6 \\
7.6 \\
7.6\end{array}$ & $\begin{array}{l}97 \\
96 \\
96 \\
96 \\
96 \\
96 \\
96 \\
96\end{array}$ \\
\hline $\begin{array}{l}11 \\
11 \\
11 \\
11 \\
11 \\
11 \\
11 \\
11\end{array}$ & $\begin{array}{l}0605 \\
0605 \\
0606 \\
0606 \\
0607 \\
0607 \\
0608 \\
0608\end{array}$ & $\begin{array}{l}0.4 \\
3.0 \\
5.0 \\
10 \\
15 \\
20 \\
25 \\
29\end{array}$ & $\begin{array}{l}600 \\
600 \\
600 \\
600 \\
600 \\
600 \\
600 \\
600\end{array}$ & $\begin{array}{l}407 \\
409 \\
410 \\
410 \\
409 \\
410 \\
406 \\
404\end{array}$ & $\begin{array}{l}7.5 \\
7.5 \\
7.5 \\
7.5 \\
7.5 \\
7.5 \\
7.5 \\
7.5\end{array}$ & $\begin{array}{l}26.5 \\
26.5 \\
26.5 \\
26.6 \\
26.6 \\
26.5 \\
26.5 \\
26.5\end{array}$ & $\begin{array}{l}-- \\
-- \\
-- \\
-- \\
- \\
- \\
-\end{array}$ & $\begin{array}{l}7.6 \\
7.6 \\
7.6 \\
7.6 \\
7.6 \\
7.6 \\
7.6 \\
7.6\end{array}$ & $\begin{array}{l}97 \\
97 \\
97 \\
97 \\
97 \\
97 \\
97 \\
97\end{array}$ \\
\hline $\begin{array}{l}11 \\
11 \\
11 \\
11 \\
11 \\
11 \\
11\end{array}$ & $\begin{array}{l}0610 \\
0610 \\
0611 \\
0611 \\
0612 \\
0612 \\
0613\end{array}$ & $\begin{array}{r}0.2 \\
3.0 \\
4.8 \\
9.8 \\
15 \\
20 \\
21\end{array}$ & 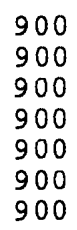 & $\begin{array}{l}410 \\
408 \\
410 \\
411 \\
407 \\
407 \\
413\end{array}$ & $\begin{array}{l}7.5 \\
7.5 \\
7.5 \\
7.5 \\
7.5 \\
7.5 \\
7.5\end{array}$ & $\begin{array}{l}26.8 \\
26.7 \\
26.8 \\
26.6 \\
26.5 \\
26.5 \\
26.5\end{array}$ & $\begin{array}{l}-- \\
-- \\
- \\
- \\
- \\
-\end{array}$ & $\begin{array}{l}7.6 \\
7.6 \\
7.6 \\
7.6 \\
7.6 \\
7.6 \\
7.6\end{array}$ & $\begin{array}{l}97 \\
97 \\
97 \\
97 \\
97 \\
97 \\
97\end{array}$ \\
\hline $\begin{array}{l}11 \\
11 \\
11 \\
11 \\
11 \\
11 \\
11 \\
11\end{array}$ & $\begin{array}{l}1528 \\
1528 \\
1529 \\
1530 \\
1530 \\
1531 \\
1531 \\
1532\end{array}$ & $\begin{array}{r}0.4 \\
3.2 \\
5.6 \\
9.9 \\
15 \\
20 \\
25 \\
27\end{array}$ & $\begin{array}{l}300 \\
300 \\
300 \\
300 \\
300 \\
300 \\
300 \\
300\end{array}$ & $\begin{array}{l}417 \\
415 \\
415 \\
417 \\
418 \\
417 \\
418 \\
417\end{array}$ & $\begin{array}{l}8.0 \\
7.8 \\
7.6 \\
7.4 \\
7.4 \\
7.4 \\
7.4 \\
7.4\end{array}$ & $\begin{array}{l}28.3 \\
27.7 \\
27.1 \\
26.4 \\
26.3 \\
26.3 \\
26.3 \\
26.3\end{array}$ & $\begin{array}{l}- \\
- \\
- \\
- \\
- \\
- \\
- \\
-\end{array}$ & $\begin{array}{l}9.2 \\
8.7 \\
8.2 \\
7.6 \\
7.5 \\
7.5 \\
7.5 \\
7.4\end{array}$ & $\begin{array}{r}120 \\
113 \\
105 \\
97 \\
95 \\
95 \\
94 \\
94\end{array}$ \\
\hline $\begin{array}{l}11 \\
11 \\
11 \\
11 \\
11 \\
11 \\
11 \\
11\end{array}$ & $\begin{array}{l}1523 \\
1523 \\
1524 \\
1524 \\
1525 \\
1525 \\
1526 \\
1526\end{array}$ & $\begin{array}{c}0.5 \\
2.9 \\
5.4 \\
9.9 \\
15 \\
20 \\
25 \\
29\end{array}$ & $\begin{array}{l}600 \\
600 \\
600 \\
600 \\
600 \\
600 \\
600 \\
600\end{array}$ & $\begin{array}{l}416 \\
416 \\
416 \\
416 \\
418 \\
416 \\
415 \\
419\end{array}$ & $\begin{array}{l}7.9 \\
7.9 \\
7.7 \\
7.7 \\
7.4 \\
7.4 \\
7.4 \\
7.4\end{array}$ & $\begin{array}{l}28.1 \\
28.2 \\
27.6 \\
27.4 \\
26.3 \\
26.3 \\
26.3 \\
26.3\end{array}$ & $\begin{array}{r}- \\
2.5 \\
- \\
- \\
- \\
\cdots \\
- \\
-\end{array}$ & $\begin{array}{l}9.0 \\
9.1 \\
8.8 \\
8.7 \\
7.6 \\
7.6 \\
7.5 \\
7.5\end{array}$ & $\begin{array}{r}117 \\
119 \\
114 \\
112 \\
96 \\
95 \\
95 \\
94\end{array}$ \\
\hline
\end{tabular}


Table 16. Water-quality data for station 401838080360701 , Ohio River at river mile 71.4 , June to October, 1995, Continued.

[ft = feet; $\mu \mathrm{s} / \mathrm{cm}=$ microsiemens per centimeter; ${ }^{\circ} \mathrm{C}=$ degrees Celsius; $\mathrm{mg} / \mathrm{I}=\mathrm{milligrams}$ par liter; $\cdot$ = data not collected]

\begin{tabular}{|c|c|c|c|c|c|c|c|c|c|}
\hline Date & Time & $\begin{array}{l}\text { Sampling } \\
\text { depth } \\
\text { (ft) }\end{array}$ & $\begin{array}{l}\text { sample } \\
\text { location } \\
\text { (ft from } \\
\text { left bank) }\end{array}$ & $\begin{array}{l}\text { Specific } \\
\text { conduct- } \\
\text { ance } \\
(\mu \mathrm{s} / \mathrm{cm})\end{array}$ & $\begin{array}{l}\text { pH } \\
\text { (stan- } \\
\text { dard } \\
\text { units) }\end{array}$ & $\begin{array}{c}\text { Temper- } \\
\text { ature, } \\
\text { water } \\
\left({ }^{\circ} \mathrm{C}\right)\end{array}$ & $\begin{array}{l}\text { Trans- } \\
\text { parency } \\
\text { (Secchi } \\
\text { disk) } \\
\text { (ft) }\end{array}$ & $\begin{array}{l}\text { Dissolved } \\
\text { oxygen } \\
\text { (mg/L) }\end{array}$ & $\begin{array}{c}\text { Dissolv } \\
\text { oxyge } \\
\text { (perce } \\
\text { satur } \\
\text { tion) }\end{array}$ \\
\hline $\begin{array}{r}\text { July } \\
11 \\
11 \\
11 \\
11 \\
11 \\
11 \\
11\end{array}$ & $\begin{array}{l}1534 \\
1534 \\
1535 \\
1535 \\
1536 \\
1537 \\
1537\end{array}$ & $\begin{array}{r}0.3 \\
3.1 \\
5.3 \\
9.9 \\
15 \\
20 \\
21\end{array}$ & $\begin{array}{l}900 \\
900 \\
900 \\
900 \\
900 \\
900 \\
900\end{array}$ & $\begin{array}{l}419 \\
419 \\
418 \\
417 \\
417 \\
417 \\
417\end{array}$ & $\begin{array}{l}7.5 \\
7.5 \\
7.5 \\
7.4 \\
7.4 \\
7.4 \\
7.4\end{array}$ & $\begin{array}{l}27.4 \\
27.3 \\
26.9 \\
26.5 \\
26.4 \\
26.3 \\
26.3\end{array}$ & $\begin{array}{l}\cdots \\
- \\
- \\
-- \\
-- \\
-\end{array}$ & $\begin{array}{l}7.8 \\
7.9 \\
7.9 \\
7.8 \\
7.7 \\
7.6 \\
7.5\end{array}$ & $\begin{array}{r}100 \\
102 \\
101 \\
98 \\
97 \\
96 \\
95\end{array}$ \\
\hline $\begin{array}{l}25 \\
25 \\
25 \\
25 \\
25 \\
25 \\
25 \\
25\end{array}$ & $\begin{array}{l}1630 \\
1630 \\
1631 \\
1631 \\
1632 \\
1632 \\
1633 \\
1633\end{array}$ & $\begin{array}{c}0.4 \\
2.9 \\
4.9 \\
9.9 \\
15 \\
20 \\
24 \\
27\end{array}$ & $\begin{array}{l}300 \\
300 \\
300 \\
300 \\
300 \\
300 \\
300 \\
300\end{array}$ & $\begin{array}{l}421 \\
421 \\
420 \\
424 \\
423 \\
421 \\
422 \\
422\end{array}$ & $\begin{array}{l}7.8 \\
7.8 \\
7.8 \\
7.6 \\
7.6 \\
7.5 \\
7.5 \\
7.5\end{array}$ & $\begin{array}{l}29.9 \\
30.0 \\
30.0 \\
29.3 \\
29.3 \\
29.1 \\
29.1 \\
29.1\end{array}$ & $\begin{array}{l}-- \\
-. \\
-- \\
-- \\
- \\
-\end{array}$ & $\begin{array}{l}8.0 \\
8.0 \\
7.8 \\
7.3 \\
7.2 \\
7.0 \\
6.9 \\
6.9\end{array}$ & $\begin{array}{r}109 \\
109 \\
106 \\
97 \\
96 \\
93 \\
92 \\
92\end{array}$ \\
\hline $\begin{array}{l}25 \\
25 \\
25 \\
25 \\
25 \\
25 \\
25 \\
25\end{array}$ & $\begin{array}{l}1620 \\
1620 \\
1621 \\
1621 \\
1622 \\
1622 \\
1623 \\
1623\end{array}$ & $\begin{array}{c}0.5 \\
3.2 \\
5.1 \\
8.8 \\
13 \\
20 \\
25 \\
30\end{array}$ & $\begin{array}{l}600 \\
600 \\
600 \\
600 \\
600 \\
600 \\
600 \\
600\end{array}$ & $\begin{array}{l}424 \\
424 \\
424 \\
424 \\
423 \\
423 \\
423 \\
423\end{array}$ & $\begin{array}{l}7.7 \\
7.7 \\
7.7 \\
7.7 \\
7.5 \\
7.5 \\
7.5 \\
7.5\end{array}$ & $\begin{array}{l}29.9 \\
29.8 \\
29.8 \\
29.7 \\
29.2 \\
29.0 \\
29.0 \\
28.9\end{array}$ & $\begin{array}{l}-- \\
- \\
-- \\
-- \\
-- \\
-\end{array}$ & $\begin{array}{l}7.6 \\
7.6 \\
7.6 \\
7.4 \\
6.8 \\
6.7 \\
6.6 \\
6.5\end{array}$ & $\begin{array}{r}102 \\
102 \\
103 \\
100 \\
91 \\
88 \\
87 \\
87\end{array}$ \\
\hline $\begin{array}{l}25 \\
25 \\
25 \\
25 \\
25 \\
25 \\
25\end{array}$ & $\begin{array}{l}1625 \\
1625 \\
1626 \\
1626 \\
1627 \\
1627 \\
1628\end{array}$ & $\begin{array}{l}0.2 \\
2.4 \\
4.6 \\
10 \\
15 \\
20 \\
24\end{array}$ & $\begin{array}{l}900 \\
900 \\
900 \\
900 \\
900 \\
900 \\
900\end{array}$ & $\begin{array}{l}424 \\
425 \\
424 \\
424 \\
424 \\
423 \\
423\end{array}$ & $\begin{array}{l}7.6 \\
7.6 \\
7.6 \\
7.5 \\
7.5 \\
7.5 \\
7.5\end{array}$ & $\begin{array}{l}29.7 \\
29.7 \\
29.6 \\
29.2 \\
29.1 \\
29.1 \\
29.0\end{array}$ & $\begin{array}{l}-- \\
-- \\
-- \\
-- \\
--\end{array}$ & $\begin{array}{l}7.1 \\
7.1 \\
7.0 \\
6.8 \\
6.7 \\
6.7 \\
6.7\end{array}$ & $\begin{array}{l}96 \\
95 \\
94 \\
90 \\
89 \\
89 \\
89\end{array}$ \\
\hline $\begin{array}{c}\text { Augus } \\
08 \\
08 \\
08 \\
08 \\
08 \\
08 \\
08 \\
08\end{array}$ & $\begin{array}{l}t \\
0547 \\
0547 \\
0548 \\
0548 \\
0549 \\
0550 \\
0550 \\
0551\end{array}$ & $\begin{array}{l}0.3 \\
3.1 \\
5.5 \\
11 \\
15 \\
20 \\
26 \\
28\end{array}$ & $\begin{array}{l}300 \\
300 \\
300 \\
300 \\
300 \\
300 \\
300 \\
300\end{array}$ & $\begin{array}{l}481 \\
480 \\
480 \\
478 \\
479 \\
481 \\
482 \\
483\end{array}$ & $\begin{array}{l}7.5 \\
7.5 \\
7.5 \\
7.5 \\
7.5 \\
7.5 \\
7.5 \\
7.5\end{array}$ & $\begin{array}{l}28.8 \\
28.8 \\
28.8 \\
28.8 \\
28.8 \\
28.8 \\
28.8 \\
28.8\end{array}$ & $\begin{array}{l}- \\
-- \\
-- \\
-- \\
-- \\
- \\
-\end{array}$ & $\begin{array}{l}7.3 \\
7.2 \\
7.3 \\
7.3 \\
7.3 \\
7.4 \\
7.2 \\
7.4\end{array}$ & $\begin{array}{l}96 \\
95 \\
96 \\
96 \\
96 \\
97 \\
95 \\
97\end{array}$ \\
\hline
\end{tabular}


Table 16. Water-quality data for station 401838080360701, Ohio River at river mile 71.4, June to October, 1995, Continued.

[ft $=$ feet $; \mu \mathrm{s} / \mathrm{cm}=$ microsiemens per centimeter; ${ }^{\circ} \mathrm{C}=$ degrees Celsius;

$\mathrm{mg} / \mathrm{L}=$ miliigrams per liter; - - = data not collected]

\begin{tabular}{|c|c|c|c|c|c|c|c|c|c|}
\hline Date & Time & $\begin{array}{l}\text { Sampling } \\
\text { depth } \\
\text { (ft) }\end{array}$ & $\begin{array}{c}\text { Sample } \\
\text { location } \\
\text { (ft from } \\
\text { left bank) }\end{array}$ & $\begin{array}{l}\text { Specific } \\
\text { conduct- } \\
\text { ance } \\
(\mu \mathrm{s} / \mathrm{cm})\end{array}$ & $\begin{array}{l}\text { pH } \\
\text { (stan- } \\
\text { dard } \\
\text { units) }\end{array}$ & $\begin{array}{c}\text { Temper- } \\
\text { ature, } \\
\text { water } \\
\left({ }^{\circ} \mathrm{C}\right)\end{array}$ & $\begin{array}{c}\text { Trans- } \\
\text { parency } \\
\text { (Secchi } \\
\text { disk) } \\
\text { (ft) }\end{array}$ & $\begin{array}{l}\text { Dissolved } \\
\text { oxygen } \\
(\mathrm{mg} / \mathrm{L})\end{array}$ & $\begin{array}{c}\text { Dissolved } \\
\text { oxygen } \\
\text { (percent } \\
\text { satura- } \\
\text { tion) }\end{array}$ \\
\hline
\end{tabular}

\begin{tabular}{|c|c|c|c|c|c|c|c|c|c|}
\hline $\begin{array}{r}\text { Augus } \\
08 \\
08 \\
08 \\
08 \\
08 \\
08 \\
08 \\
08\end{array}$ & $\begin{array}{l}t \\
0554 \\
0554 \\
0555 \\
0555 \\
0556 \\
0556 \\
0557 \\
0557\end{array}$ & $\begin{array}{c}0.5 \\
3.2 \\
5.4 \\
10 \\
15 \\
20 \\
25 \\
30\end{array}$ & $\begin{array}{l}600 \\
600 \\
600 \\
600 \\
600 \\
600 \\
600 \\
600\end{array}$ & $\begin{array}{l}477 \\
478 \\
479 \\
478 \\
476 \\
479 \\
472 \\
472\end{array}$ & $\begin{array}{l}7.5 \\
7.5 \\
7.5 \\
7.5 \\
7.5 \\
7.5 \\
7.5 \\
7.5\end{array}$ & $\begin{array}{l}28.9 \\
28.9 \\
28.9 \\
28.8 \\
28.8 \\
28.8 \\
28.8 \\
28.8\end{array}$ & $\begin{array}{l}\cdots \\
- \\
\cdots \\
\cdots \\
- \\
-\end{array}$ & $\begin{array}{l}7.5 \\
7.4 \\
7.4 \\
7.2 \\
7.4 \\
7.3 \\
7.3 \\
7.4\end{array}$ & $\begin{array}{l}99 \\
97 \\
98 \\
95 \\
97 \\
96 \\
96 \\
98\end{array}$ \\
\hline $\begin{array}{l}08 \\
08 \\
08 \\
08 \\
08 \\
08 \\
08 \\
08\end{array}$ & $\begin{array}{l}0559 \\
0559 \\
0600 \\
0600 \\
0601 \\
0601 \\
0602 \\
0602\end{array}$ & $\begin{array}{c}0.2 \\
3.3 \\
5.4 \\
10 \\
15 \\
20 \\
25 \\
26\end{array}$ & $\begin{array}{l}900 \\
900 \\
900 \\
900 \\
900 \\
900 \\
900 \\
900\end{array}$ & $\begin{array}{l}478 \\
478 \\
479 \\
477 \\
473 \\
479 \\
473 \\
481\end{array}$ & $\begin{array}{l}7.5 \\
7.5 \\
7.5 \\
7.5 \\
7.5 \\
7.5 \\
7.5 \\
7.5\end{array}$ & $\begin{array}{l}28.9 \\
28.9 \\
28.9 \\
28.9 \\
28.9 \\
28.9 \\
28.9 \\
28.9\end{array}$ & $\begin{array}{l}- \\
-- \\
- \\
- \\
- \\
- \\
-\end{array}$ & $\begin{array}{l}7.3 \\
7.3 \\
7.3 \\
7.4 \\
7.4 \\
7.4 \\
7.4 \\
7.3\end{array}$ & $\begin{array}{l}97 \\
96 \\
97 \\
97 \\
97 \\
97 \\
97 \\
96\end{array}$ \\
\hline $\begin{array}{l}08 \\
08 \\
08 \\
08 \\
08 \\
08 \\
08 \\
08\end{array}$ & $\begin{array}{l}1356 \\
1356 \\
1357 \\
1357 \\
1358 \\
1358 \\
1359 \\
1359\end{array}$ & $\begin{array}{c}0.4 \\
3.2 \\
5.2 \\
9.8 \\
15 \\
20 \\
25 \\
27\end{array}$ & $\begin{array}{l}300 \\
300 \\
300 \\
300 \\
300 \\
300 \\
300 \\
300\end{array}$ & $\begin{array}{l}472 \\
473 \\
473 \\
471 \\
470 \\
470 \\
476 \\
469\end{array}$ & $\begin{array}{l}7.6 \\
7.5 \\
7.5 \\
7.5 \\
7.5 \\
7.5 \\
7.5 \\
7.5\end{array}$ & $\begin{array}{l}28.5 \\
28.6 \\
28.6 \\
28.6 \\
28.6 \\
28.6 \\
28.6 \\
28.6\end{array}$ & $\begin{array}{l}-- \\
- \\
- \\
- \\
- \\
- \\
- \\
-\end{array}$ & $\begin{array}{l}7.2 \\
7.0 \\
7.0 \\
6.9 \\
6.9 \\
6.9 \\
7.0 \\
7.0\end{array}$ & $\begin{array}{l}95 \\
92 \\
92 \\
91 \\
91 \\
91 \\
92 \\
91\end{array}$ \\
\hline $\begin{array}{l}08 \\
08 \\
08 \\
08 \\
08 \\
08 \\
08 \\
08\end{array}$ & $\begin{array}{l}1346 \\
1346 \\
1347 \\
1347 \\
1348 \\
1348 \\
1349 \\
1349\end{array}$ & $\begin{array}{r}0.7 \\
3.1 \\
5.1 \\
9.9 \\
15 \\
20 \\
25 \\
29\end{array}$ & $\begin{array}{l}600 \\
600 \\
600 \\
600 \\
600 \\
600 \\
600 \\
600\end{array}$ & $\begin{array}{l}468 \\
469 \\
469 \\
469 \\
469 \\
469 \\
469 \\
470\end{array}$ & $\begin{array}{l}7.7 \\
7.6 \\
7.6 \\
7.6 \\
7.5 \\
7.5 \\
7.5 \\
7.5\end{array}$ & $\begin{array}{l}28.7 \\
28.6 \\
28.7 \\
28.6 \\
28.6 \\
28.6 \\
28.6 \\
28.6\end{array}$ & $\begin{array}{c}- \\
- \\
3.5 \\
- \\
- \\
- \\
- \\
-\end{array}$ & $\begin{array}{l}7.3 \\
7.1 \\
7.1 \\
7.0 \\
7.0 \\
7.0 \\
7.0 \\
7.1\end{array}$ & $\begin{array}{l}95 \\
94 \\
93 \\
92 \\
92 \\
92 \\
92 \\
92\end{array}$ \\
\hline $\begin{array}{l}08 \\
08 \\
08 \\
08 \\
08 \\
08 \\
08\end{array}$ & $\begin{array}{l}1351 \\
1351 \\
1352 \\
1352 \\
1353 \\
1353 \\
1354\end{array}$ & $\begin{array}{r}0.4 \\
3.2 \\
5.0 \\
9.7 \\
15 \\
20 \\
24\end{array}$ & $\begin{array}{l}900 \\
900 \\
900 \\
900 \\
900 \\
900 \\
900\end{array}$ & $\begin{array}{l}469 \\
470 \\
469 \\
469 \\
469 \\
467 \\
466\end{array}$ & $\begin{array}{l}7.5 \\
7.5 \\
7.5 \\
7.5 \\
7.5 \\
7.5 \\
7.5\end{array}$ & $\begin{array}{l}29.1 \\
29.0 \\
29.0 \\
28.9 \\
28.7 \\
28.7 \\
28.7\end{array}$ & $\begin{array}{l}\cdots \\
\cdots \\
\cdots \\
\cdots \\
\cdots\end{array}$ & $\begin{array}{l}7.0 \\
6.9 \\
6.9 \\
7.0 \\
6.9 \\
6.9 \\
7.1\end{array}$ & $\begin{array}{l}93 \\
91 \\
91 \\
92 \\
91 \\
91 \\
93\end{array}$ \\
\hline
\end{tabular}


Table 16. Water-quality data for station 401838080360701, Ohio River at river mile 71.4, June to October, 1995, Continued.

[ft = feet; $\mu \mathrm{s} / \mathrm{cm}=$ microsiemens per centimeter; ${ }^{\circ} \mathrm{C}=$ degrees Celsius; $\mathrm{mg} / \mathrm{L}=\mathrm{mili}$ igrams per liter; $\cdots$ = data not collected]

\begin{tabular}{|c|c|c|c|c|c|c|c|c|c|}
\hline Date & Time & $\begin{array}{l}\text { Sampling } \\
\text { depth } \\
\text { (ft) }\end{array}$ & $\begin{array}{c}\text { Sarmle } \\
\text { location } \\
\text { (Et Erom } \\
\text { left bank) }\end{array}$ & $\begin{array}{l}\text { Specific } \\
\text { conduct - } \\
\text { ance } \\
(\mu \mathrm{s} / \mathrm{cm})\end{array}$ & $\begin{array}{l}\mathrm{pH} \\
\text { (stan- } \\
\text { dard } \\
\text { units) }\end{array}$ & $\begin{array}{c}\text { Termer- } \\
\text { ature, } \\
\text { water } \\
\left({ }^{\circ} \mathrm{C}\right)\end{array}$ & $\begin{array}{c}\text { Trans- } \\
\text { parency } \\
\text { (Secchi } \\
\text { disk) } \\
\text { (ft) }\end{array}$ & $\begin{array}{l}\text { Dissolved } \\
\text { oxygen } \\
\text { (mg/L) }\end{array}$ & $\begin{array}{c}\text { Dissolved } \\
\text { oxygen } \\
\text { (percent } \\
\text { satura- } \\
\text { tion) }\end{array}$ \\
\hline
\end{tabular}

\begin{tabular}{|c|c|c|c|c|c|c|c|c|c|}
\hline $\begin{array}{c}\text { Augus } \\
22 \\
22 \\
22 \\
22 \\
22 \\
22 \\
22 \\
22\end{array}$ & $\begin{array}{l}0613 \\
0613 \\
0614 \\
0614 \\
0615 \\
0615 \\
0616 \\
0616\end{array}$ & $\begin{array}{l}0.9 \\
3.2 \\
5.1 \\
9.8 \\
15 \\
20 \\
25 \\
27\end{array}$ & $\begin{array}{l}300 \\
300 \\
300 \\
300 \\
300 \\
300 \\
300 \\
300\end{array}$ & $\begin{array}{l}512 \\
513 \\
513 \\
515 \\
515 \\
512 \\
516 \\
514\end{array}$ & $\begin{array}{l}7.8 \\
7.8 \\
7.8 \\
7.8 \\
7.8 \\
7.8 \\
7.8 \\
7.8\end{array}$ & $\begin{array}{l}30.7 \\
30.7 \\
30.7 \\
30.7 \\
30.7 \\
30.7 \\
30.7 \\
30.7\end{array}$ & $\begin{array}{l}- \\
- \\
-- \\
-- \\
-- \\
- \\
--\end{array}$ & $\begin{array}{l}7.2 \\
7.2 \\
7.2 \\
7.1 \\
7.2 \\
7.1 \\
7.0 \\
7.1\end{array}$ & $\begin{array}{l}98 \\
98 \\
99 \\
97 \\
99 \\
98 \\
96 \\
96\end{array}$ \\
\hline $\begin{array}{l}22 \\
22 \\
22 \\
22 \\
22 \\
22 \\
22 \\
22\end{array}$ & $\begin{array}{l}0618 \\
0618 \\
0619 \\
0619 \\
0620 \\
0620 \\
0621 \\
0621\end{array}$ & $\begin{array}{l}0.9 \\
3.0 \\
5.1 \\
10 \\
15 \\
20 \\
25 \\
28\end{array}$ & $\begin{array}{l}600 \\
600 \\
600 \\
600 \\
600 \\
600 \\
600 \\
600\end{array}$ & $\begin{array}{l}516 \\
518 \\
515 \\
514 \\
514 \\
516 \\
513 \\
513\end{array}$ & $\begin{array}{l}7.8 \\
7.8 \\
7.8 \\
7.8 \\
7.8 \\
7.8 \\
7.7 \\
7.7\end{array}$ & $\begin{array}{l}30.8 \\
30.9 \\
30.9 \\
30.8 \\
30.7 \\
30.7 \\
30.7 \\
30.7\end{array}$ & $\begin{array}{l}-- \\
-- \\
-- \\
-- \\
-- \\
--\end{array}$ & $\begin{array}{l}7.2 \\
7.2 \\
7.2 \\
7.2 \\
7.2 \\
7.2 \\
7.2 \\
7.1\end{array}$ & $\begin{array}{l}99 \\
98 \\
98 \\
99 \\
98 \\
98 \\
98 \\
97\end{array}$ \\
\hline $\begin{array}{l}22 \\
22 \\
22 \\
22 \\
22 \\
22 \\
22\end{array}$ & $\begin{array}{l}0623 \\
0623 \\
0624 \\
0624 \\
0625 \\
0625 \\
0626\end{array}$ & $\begin{array}{l}0.4 \\
3.2 \\
5.2 \\
9.8 \\
15 \\
20 \\
25\end{array}$ & $\begin{array}{l}900 \\
900 \\
900 \\
900 \\
900 \\
900 \\
900\end{array}$ & $\begin{array}{l}513 \\
513 \\
517 \\
514 \\
516 \\
514 \\
513\end{array}$ & $\begin{array}{l}7.8 \\
7.8 \\
7.8 \\
7.8 \\
7.8 \\
7.7 \\
7.7\end{array}$ & $\begin{array}{l}30.8 \\
30.9 \\
30.9 \\
30.9 \\
30.8 \\
30.7 \\
30.7\end{array}$ & $\begin{array}{l}-- \\
-- \\
- \\
- \\
- \\
-\end{array}$ & $\begin{array}{l}7.2 \\
7.1 \\
7.2 \\
7.2 \\
7.2 \\
7.2 \\
7.2\end{array}$ & $\begin{array}{l}98 \\
98 \\
98 \\
98 \\
99 \\
98 \\
98\end{array}$ \\
\hline $\begin{array}{l}22 \\
22 \\
22 \\
22 \\
22 \\
22 \\
22 \\
22\end{array}$ & $\begin{array}{l}1527 \\
1527 \\
1528 \\
1528 \\
1529 \\
1530 \\
1530 \\
1531\end{array}$ & $\begin{array}{l}0.5 \\
3.1 \\
5.5 \\
9.8 \\
15 \\
19 \\
26 \\
28\end{array}$ & $\begin{array}{l}300 \\
300 \\
300 \\
300 \\
300 \\
300 \\
300 \\
300\end{array}$ & $\begin{array}{l}509 \\
509 \\
509 \\
509 \\
510 \\
511 \\
512 \\
512\end{array}$ & $\begin{array}{l}8.2 \\
8.2 \\
8.2 \\
8.1 \\
8.1 \\
7.9 \\
7.9 \\
7.9\end{array}$ & $\begin{array}{l}30.8 \\
30.8 \\
30.8 \\
30.8 \\
30.7 \\
30.7 \\
30.6 \\
30.6\end{array}$ & $\begin{array}{l}- \\
- \\
- \\
- \\
- \\
- \\
-\end{array}$ & $\begin{array}{l}8.6 \\
8.6 \\
8.7 \\
8.5 \\
8.3 \\
8.0 \\
7.9 \\
7.8\end{array}$ & $\begin{array}{l}117 \\
116 \\
118 \\
115 \\
113 \\
108 \\
107 \\
106\end{array}$ \\
\hline $\begin{array}{l}22 \\
22 \\
22 \\
22 \\
22 \\
22 \\
22 \\
22\end{array}$ & $\begin{array}{l}1521 \\
1521 \\
1522 \\
1522 \\
1523 \\
1524 \\
1524 \\
1525\end{array}$ & $\begin{array}{c}0.3 \\
3.2 \\
4.3 \\
8.7 \\
17 \\
21 \\
24 \\
27\end{array}$ & $\begin{array}{l}600 \\
600 \\
600 \\
600 \\
600 \\
600 \\
600 \\
600\end{array}$ & $\begin{array}{l}513 \\
513 \\
511 \\
511 \\
510 \\
513 \\
520 \\
543\end{array}$ & $\begin{array}{l}8.0 \\
8.0 \\
8.0 \\
8.0 \\
8.0 \\
7.9 \\
7.8 \\
7.7\end{array}$ & $\begin{array}{l}31.1 \\
31.1 \\
31.0 \\
30.7 \\
30.7 \\
30.7 \\
30.6 \\
30.2\end{array}$ & $\begin{array}{c}\ldots \\
3.5 \\
\therefore \\
\therefore \\
\therefore \\
-\end{array}$ & $\begin{array}{l}7.9 \\
8.1 \\
8.1 \\
8.2 \\
8.2 \\
7.9 \\
7.7 \\
7.0\end{array}$ & $\begin{array}{r}108 \\
110 \\
110 \\
111 \\
111 \\
107 \\
104 \\
95\end{array}$ \\
\hline
\end{tabular}


Table 16. Water-quality data for station 401838080360701 , Ohio River at river mile 71.4, June to October, 1995, Continued.

[ft $=$ feet; $\mu \mathrm{s} / \mathrm{cm}=$ microsiemens per centimeter; ${ }^{\circ} \mathrm{C}=$ degrees Celsius; $\mathrm{mg} / \mathrm{L}=$ miligrams per liter; $-\cdot$ = data not collected]

\begin{tabular}{|c|c|c|c|c|c|c|c|c|c|}
\hline Date & Time & $\begin{array}{l}\text { Sampling } \\
\text { depth } \\
(\mathrm{ft})\end{array}$ & $\begin{array}{c}\text { Sample } \\
\text { location } \\
\text { (ft from } \\
\text { left bank) }\end{array}$ & $\begin{array}{l}\text { specific } \\
\text { conduct- } \\
\text { ance } \\
(\mu \mathrm{s} / \mathrm{cm})\end{array}$ & $\begin{array}{l}\text { pH } \\
\text { (stan- } \\
\text { dard } \\
\text { units) }\end{array}$ & $\begin{array}{c}\text { Temper- } \\
\text { ature, } \\
\text { water } \\
\left({ }^{\circ} \mathrm{C}\right)\end{array}$ & $\begin{array}{l}\text { Trans- } \\
\text { parency } \\
\text { (Secchi } \\
\text { disk) } \\
\text { (ft) }\end{array}$ & $\begin{array}{l}\text { Dissolved } \\
\text { oxygen } \\
(\mathrm{mg} / \mathrm{L})\end{array}$ & $\begin{array}{c}\text { Dissolved } \\
\text { oxygen } \\
\text { (percent } \\
\text { satura- } \\
\text { tion) }\end{array}$ \\
\hline
\end{tabular}

\begin{tabular}{|c|c|c|c|c|c|c|c|c|c|}
\hline $\begin{array}{r}\text { Augu } \\
22 \\
22 \\
22 \\
22 \\
22 \\
22 \\
22\end{array}$ & $\begin{array}{l}\text { t } 1533 \\
1533 \\
1534 \\
1535 \\
1535 \\
1536 \\
1536\end{array}$ & $\begin{array}{c}0.5 \\
3.0 \\
4.9 \\
10 \\
15 \\
20 \\
24\end{array}$ & $\begin{array}{l}900 \\
900 \\
900 \\
900 \\
900 \\
900 \\
900\end{array}$ & $\begin{array}{l}513 \\
511 \\
511 \\
511 \\
516 \\
510 \\
510\end{array}$ & $\begin{array}{l}7.8 \\
7.9 \\
7.9 \\
7.9 \\
7.8 \\
7.8 \\
7.8\end{array}$ & $\begin{array}{l}31.0 \\
30.8 \\
30.8 \\
30.7 \\
30.7 \\
30.7 \\
30.7\end{array}$ & $\begin{array}{l}- \\
\cdots \\
- \\
- \\
\cdots \\
-\end{array}$ & $\begin{array}{l}7.9 \\
7.7 \\
7.7 \\
7.7 \\
7.7 \\
7.7 \\
7.7\end{array}$ & $\begin{array}{l}107 \\
105 \\
104 \\
105 \\
104 \\
104 \\
104\end{array}$ \\
\hline $\begin{array}{r}\text { Sept } \\
07 \\
07 \\
07 \\
07 \\
07 \\
07 \\
07 \\
07\end{array}$ & $\begin{array}{l}\text { nber } \\
1401 \\
1401 \\
1402 \\
1402 \\
1403 \\
1403 \\
1404 \\
1405\end{array}$ & $\begin{array}{l}0.2 \\
3.0 \\
5.0 \\
10 \\
15 \\
20 \\
25 \\
27\end{array}$ & $\begin{array}{l}300 \\
300 \\
300 \\
300 \\
300 \\
300 \\
300 \\
300\end{array}$ & $\begin{array}{l}484 \\
483 \\
483 \\
482 \\
480 \\
479 \\
480 \\
480\end{array}$ & $\begin{array}{l}8.1 \\
8.1 \\
8.1 \\
8.2 \\
8.2 \\
8.1 \\
8.1 \\
8.1\end{array}$ & $\begin{array}{l}29.0 \\
29.0 \\
29.0 \\
28.9 \\
28.7 \\
28.5 \\
28.5 \\
28.4\end{array}$ & $\begin{array}{l}-- \\
-- \\
- \\
-- \\
-- \\
-\end{array}$ & $\begin{array}{l}7.7 \\
7.7 \\
7.7 \\
7.9 \\
7.9 \\
7.8 \\
7.8 \\
7.4\end{array}$ & $\begin{array}{r}102 \\
102 \\
103 \\
104 \\
105 \\
104 \\
102 \\
97\end{array}$ \\
\hline $\begin{array}{l}07 \\
07 \\
07 \\
07 \\
07 \\
07 \\
07 \\
07\end{array}$ & $\begin{array}{l}1407 \\
1407 \\
1408 \\
1408 \\
1409 \\
1409 \\
1410 \\
1410\end{array}$ & $\begin{array}{c}0.2 \\
3.0 \\
5.0 \\
10 \\
15 \\
20 \\
25 \\
29\end{array}$ & $\begin{array}{l}600 \\
600 \\
600 \\
600 \\
600 \\
600 \\
600 \\
600\end{array}$ & $\begin{array}{l}483 \\
482 \\
483 \\
482 \\
480 \\
488 \\
480 \\
481\end{array}$ & $\begin{array}{l}8.1 \\
8.1 \\
8.1 \\
8.1 \\
8.2 \\
8.1 \\
8.0 \\
7.9\end{array}$ & $\begin{array}{l}28.9 \\
28.9 \\
28.8 \\
28.8 \\
28.6 \\
28.5 \\
28.4 \\
28.4\end{array}$ & $\begin{array}{l}-- \\
- \\
-- \\
- \\
- \\
- \\
-\end{array}$ & $\begin{array}{l}7.7 \\
7.7 \\
7.8 \\
7.8 \\
7.8 \\
7.7 \\
7.3 \\
7.1\end{array}$ & $\begin{array}{r}102 \\
102 \\
103 \\
103 \\
103 \\
101 \\
96 \\
93\end{array}$ \\
\hline $\begin{array}{l}07 \\
07 \\
07 \\
07 \\
07 \\
07 \\
07 \\
07\end{array}$ & $\begin{array}{l}1414 \\
1414 \\
1415 \\
1415 \\
1416 \\
1416 \\
1417 \\
1417\end{array}$ & $\begin{array}{l}0.2 \\
3.0 \\
5.0 \\
10 \\
15 \\
20 \\
25 \\
29\end{array}$ & $\begin{array}{l}900 \\
900 \\
900 \\
900 \\
900 \\
900 \\
900 \\
900\end{array}$ & $\begin{array}{l}484 \\
485 \\
483 \\
483 \\
481 \\
482 \\
482 \\
482\end{array}$ & $\begin{array}{l}8.1 \\
8.1 \\
8.1 \\
8.1 \\
8.1 \\
8.0 \\
8.0 \\
8.0\end{array}$ & $\begin{array}{l}28.8 \\
28.8 \\
28.8 \\
28.6 \\
28.5 \\
28.4 \\
28.4 \\
28.4\end{array}$ & $\begin{array}{l}-- \\
- \\
- \\
- \\
-- \\
-\end{array}$ & $\begin{array}{l}7.7 \\
7.7 \\
7.6 \\
7.6 \\
7.7 \\
7.5 \\
7.5 \\
7.2\end{array}$ & $\begin{array}{r}102 \\
102 \\
101 \\
101 \\
101 \\
99 \\
98 \\
95\end{array}$ \\
\hline $\begin{array}{l}08 \\
08 \\
08 \\
08 \\
08 \\
08 \\
08 \\
08 \\
08\end{array}$ & $\begin{array}{l}0540 \\
0540 \\
0541 \\
0541 \\
0542 \\
0542 \\
0543 \\
0543 \\
0544\end{array}$ & $\begin{array}{l}1.0 \\
2.9 \\
5.5 \\
9.6 \\
15 \\
20 \\
24 \\
30 \\
33\end{array}$ & $\begin{array}{l}300 \\
300 \\
300 \\
300 \\
300 \\
300 \\
300 \\
300 \\
300\end{array}$ & $\begin{array}{l}476 \\
471 \\
472 \\
474 \\
474 \\
474 \\
477 \\
469 \\
479\end{array}$ & $\begin{array}{l}7.9 \\
7.9 \\
7.9 \\
7.9 \\
7.9 \\
7.9 \\
7.9 \\
7.9 \\
7.9\end{array}$ & $\begin{array}{l}28.3 \\
28.3 \\
28.3 \\
28.3 \\
28.3 \\
28.3 \\
28.3 \\
28.3 \\
28.3\end{array}$ & $\begin{array}{l}\cdots \\
\cdots \\
\cdots \\
\cdots \\
\cdots \\
\cdots\end{array}$ & $\begin{array}{l}7.6 \\
7.6 \\
7.5 \\
7.6 \\
7.6 \\
7.5 \\
7.5 \\
7.5 \\
7.4\end{array}$ & $\begin{array}{r}100 \\
100 \\
98 \\
99 \\
99 \\
98 \\
98 \\
99 \\
97\end{array}$ \\
\hline
\end{tabular}


Table 16. Water-quality data for station 401838080360701, Ohio River at river mile 71.4, June to October, 1995, Continued.

[ft $=$ feet; $\mu \mathrm{s} / \mathrm{cm}=$ microsiemens per centimeter; ${ }^{\circ} \mathrm{C}=$ degrees Celsius; $\mathrm{mg} / \mathrm{L}=\mathrm{mili}$ igrams per liter; - = data not collected]

\begin{tabular}{|c|c|c|c|c|c|c|c|c|c|}
\hline Date & Time & $\begin{array}{l}\text { Sampling } \\
\text { depth } \\
\text { (ft) }\end{array}$ & $\begin{array}{c}\text { Sample } \\
\text { location } \\
\text { (ft Erom } \\
\text { left bank) }\end{array}$ & $\begin{array}{l}\text { Specific } \\
\text { conduct- } \\
\text { ance } \\
(\mu \mathrm{s} / \mathrm{cm})\end{array}$ & $\begin{array}{l}\mathrm{pH} \\
\text { (stan- } \\
\text { dard } \\
\text { units) }\end{array}$ & $\begin{array}{l}\text { Temper- } \\
\text { ature, } \\
\text { water } \\
\left({ }^{\circ} \mathrm{C}\right)\end{array}$ & $\begin{array}{l}\text { Trans- } \\
\text { parency } \\
\text { (secchi } \\
\text { disk) } \\
\text { (ft) }\end{array}$ & $\begin{array}{l}\text { Dissolved } \\
\text { oxygen } \\
\text { (mg/L) }\end{array}$ & $\begin{array}{c}\text { Dissolved } \\
\text { oxygen } \\
\text { (percent } \\
\text { satura- } \\
\text { tion) }\end{array}$ \\
\hline
\end{tabular}

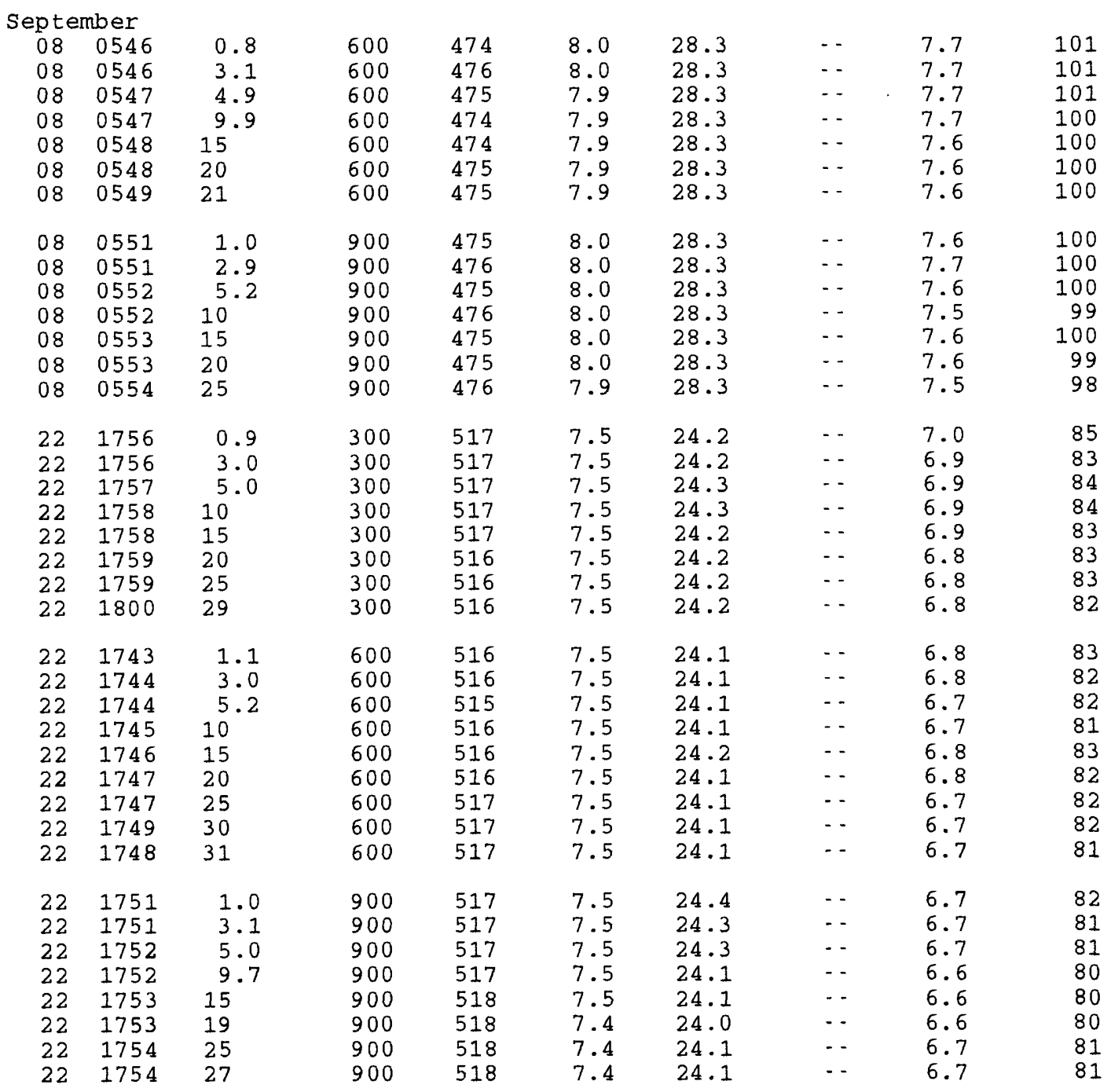


Table 16. Water-quality data for station 401838080360701 , Ohio River at river mile 71.4, June to October, 1995, Continued.

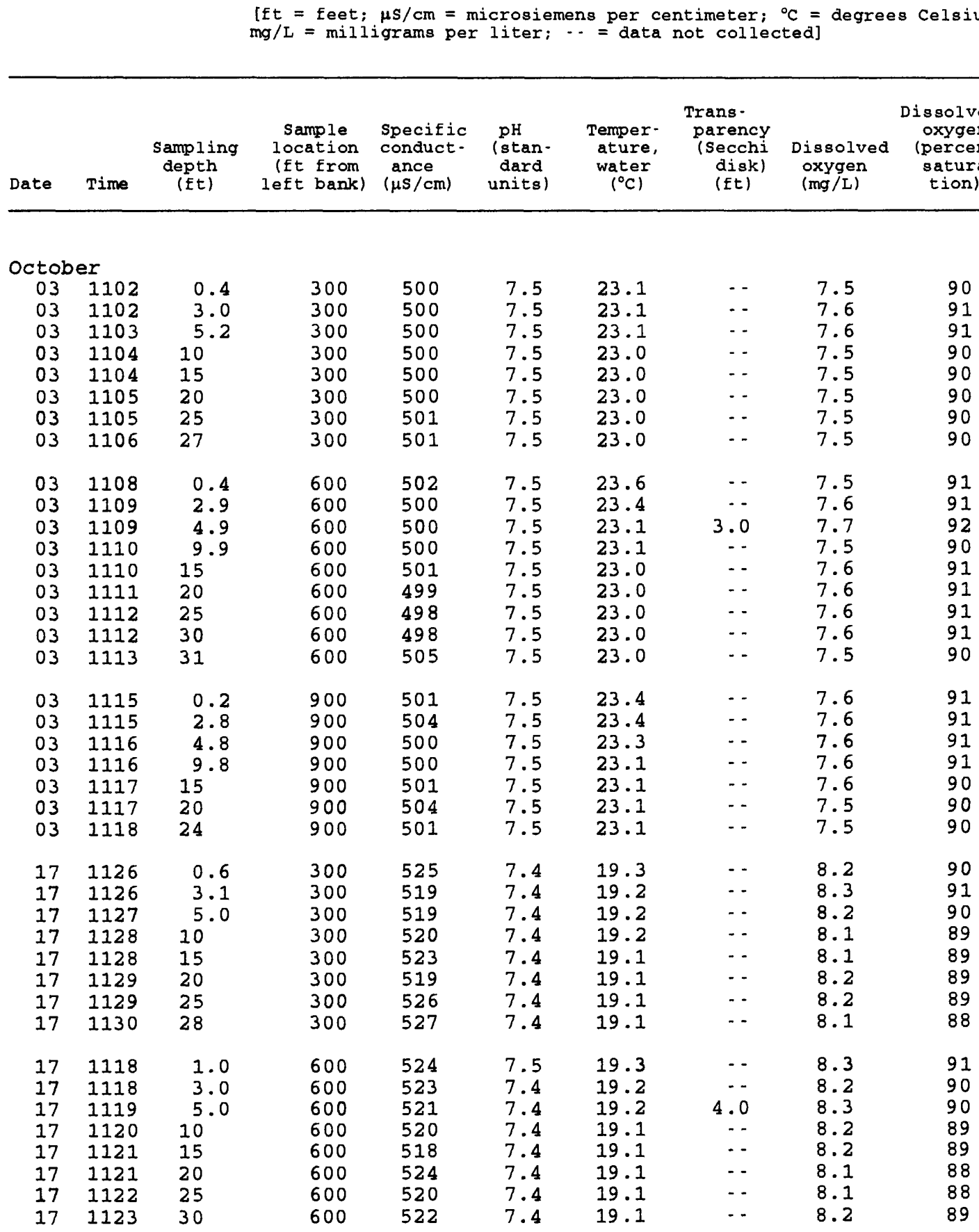


Table 16. Water-quality data for station 401838080360701 , Ohio River at river mile 71.4, June to October, 1995, Continued.

\begin{tabular}{|c|c|c|c|c|c|c|c|c|c|}
\hline Date & Time & $\begin{array}{l}\text { Sampling } \\
\text { depth } \\
\text { (ft) }\end{array}$ & $\begin{array}{c}\text { Sample } \\
\text { location } \\
\text { (ft from } \\
\text { left bank) }\end{array}$ & $\begin{array}{l}\text { Specific } \\
\text { conduct- } \\
\text { ance } \\
(\mu \mathrm{S} / \mathrm{cm})\end{array}$ & $\begin{array}{l}\mathrm{pH} \\
\text { (stan- } \\
\text { dard } \\
\text { units) }\end{array}$ & $\begin{array}{c}\text { Temper- } \\
\text { ature, } \\
\text { water } \\
\left({ }^{\circ} \mathrm{C}\right)\end{array}$ & $\begin{array}{l}\text { Trans- } \\
\text { parency } \\
\text { (secchi } \\
\text { disk) } \\
\text { (ft) }\end{array}$ & $\begin{array}{l}\text { Dissolved } \\
\text { oxygen } \\
(\mathrm{mg} / \mathrm{L})\end{array}$ & $\begin{array}{c}\text { Dissolved } \\
\text { oxygen } \\
\text { (percent } \\
\text { satura- } \\
\text { tion) }\end{array}$ \\
\hline \multicolumn{10}{|c|}{ October } \\
\hline 17 & 1136 & 0.8 & 900 & 523 & 7.5 & 19.3 & $\cdots$ & 8.3 & 90 \\
\hline 17 & 1136 & 3.1 & 900 & 520 & 7.4 & 19.4 & $\cdots$ & 8.1 & 89 \\
\hline 17 & 1137 & 4.8 & 900 & 524 & 7.4 & 19.3 & $\cdots$ & 8.2 & 90 \\
\hline 17 & 1137 & 10 & 900 & 520 & 7.4 & 19.3 & $\cdots$ & 8.2 & 90 \\
\hline 17 & 1138 & 15 & 900 & 519 & 7.4 & 19.1 & $\cdots$ & 8.3 & 90 \\
\hline 17 & 1138 & 20 & 900 & 524 & 7.4 & 19.1 & $\cdots$ & 8.3 & 91 \\
\hline 17 & 1139 & 21 & 900 & 523 & 7.4 & 19.1 & $\cdots$ & 8.3 & 90 \\
\hline
\end{tabular}


Table 17. Water-quality data for station 401728080365101, Ohio River at river mile 72.9 , June to october, 1995.

ft $=$ feet $; \mu \mathrm{s} / \mathrm{cm}=$ microsiemens per centimeter; ${ }^{\circ} \mathrm{C}=$ degrees Celsius; $\mathrm{mg} / \mathrm{L}=$ milligrams per liter; -. = data not collected]

\begin{tabular}{|c|c|c|c|c|c|c|c|c|c|}
\hline Date & Time & $\begin{array}{l}\text { Sampling } \\
\text { depth } \\
\text { (ft) }\end{array}$ & $\begin{array}{l}\text { Sample } \\
\text { location } \\
\text { (ft from } \\
\text { left bank) }\end{array}$ & $\begin{array}{l}\text { Specific } \\
\text { conduct- } \\
\text { ance } \\
(\mu \mathrm{S} / \mathrm{cm})\end{array}$ & $\begin{array}{l}\mathrm{pH} \\
\text { (stan- } \\
\text { dard } \\
\text { units) }\end{array}$ & $\begin{array}{c}\text { Temper- } \\
\text { ature, } \\
\text { water } \\
\left({ }^{\circ} \mathrm{C}\right)\end{array}$ & $\begin{array}{l}\text { Trans- } \\
\text { parency } \\
\text { (Secchi } \\
\text { disk) } \\
\text { (ft) }\end{array}$ & $\begin{array}{l}\text { Dissolved } \\
\text { oxygen } \\
(\mathrm{mg} / \mathrm{L})\end{array}$ & $\begin{array}{c}\text { Dissolved } \\
\text { oxygen } \\
\text { (percent } \\
\text { satura- } \\
\text { tion) }\end{array}$ \\
\hline $\begin{array}{r}\text { July } \\
11 \\
11 \\
11 \\
11\end{array}$ & $\begin{array}{l}1541 \\
1541 \\
1543 \\
1542\end{array}$ & $\begin{array}{r}0.6 \\
3.1 \\
22 \\
45\end{array}$ & $\begin{array}{l}600 \\
600 \\
600 \\
600\end{array}$ & $\begin{array}{l}416 \\
419 \\
423 \\
418\end{array}$ & $\begin{array}{l}8.0 \\
7.6 \\
7.4 \\
7.4\end{array}$ & $\begin{array}{l}28.1 \\
27.0 \\
26.5 \\
26.5\end{array}$ & $\begin{array}{l}-- \\
\cdots \\
--\end{array}$ & $\begin{array}{l}9.3 \\
8.2 \\
7.5 \\
7.3\end{array}$ & $\begin{array}{r}122 \\
105 \\
95 \\
93\end{array}$ \\
\hline $\begin{array}{l}25 \\
25 \\
25 \\
25\end{array}$ & $\begin{array}{l}1637 \\
1637 \\
1639 \\
1638\end{array}$ & $\begin{array}{l}0.5 \\
3.3 \\
12 \\
23\end{array}$ & $\begin{array}{l}600 \\
600 \\
600 \\
600\end{array}$ & $\begin{array}{l}427 \\
428 \\
429 \\
431\end{array}$ & $\begin{array}{l}7.7 \\
7.7 \\
7.5 \\
7.5\end{array}$ & $\begin{array}{l}29.7 \\
29.6 \\
29.1 \\
29.0\end{array}$ & $\begin{array}{l}\cdots \\
-- \\
--\end{array}$ & $\begin{array}{l}7.7 \\
7.5 \\
6.6 \\
6.6\end{array}$ & $\begin{array}{r}103 \\
100 \\
88 \\
88\end{array}$ \\
\hline $\begin{array}{c}\text { Augus } \\
08 \\
08 \\
08 \\
08\end{array}$ & $\begin{array}{l}\text { t } \\
1401 \\
1401 \\
1403 \\
1402\end{array}$ & $\begin{array}{l}0.4 \\
3.3 \\
14 \\
28\end{array}$ & $\begin{array}{l}600 \\
600 \\
600 \\
600\end{array}$ & $\begin{array}{l}469 \\
469 \\
470 \\
472\end{array}$ & $\begin{array}{l}7.6 \\
7.5 \\
7.5 \\
7.5\end{array}$ & $\begin{array}{l}28.6 \\
28.7 \\
28.7 \\
28.6\end{array}$ & $\begin{array}{l}-- \\
-- \\
--\end{array}$ & $\begin{array}{l}7.1 \\
7.1 \\
6.9 \\
6.8\end{array}$ & $\begin{array}{l}93 \\
94 \\
91 \\
90\end{array}$ \\
\hline $\begin{array}{l}22 \\
22 \\
22 \\
22\end{array}$ & $\begin{array}{l}1538 \\
1538 \\
1540 \\
1539\end{array}$ & $\begin{array}{l}0.9 \\
3.1 \\
12 \\
25\end{array}$ & $\begin{array}{l}600 \\
600 \\
600 \\
600\end{array}$ & $\begin{array}{l}518 \\
517 \\
519 \\
528\end{array}$ & $\begin{array}{l}8.0 \\
8.0 \\
7.9 \\
7.9\end{array}$ & $\begin{array}{l}30.6 \\
30.6 \\
30.6 \\
30.6\end{array}$ & $\begin{array}{l}-- \\
-- \\
--\end{array}$ & $\begin{array}{l}7.9 \\
8.0 \\
7.7 \\
7.7\end{array}$ & $\begin{array}{l}108 \\
108 \\
104 \\
104\end{array}$ \\
\hline $\begin{array}{c}\text { Septe } \\
07 \\
07 \\
07 \\
07\end{array}$ & $\begin{array}{l}\text { nber } \\
1356 \\
1357 \\
1358 \\
1357\end{array}$ & $\begin{array}{l}0.2 \\
3.0 \\
14 \\
29\end{array}$ & $\begin{array}{l}600 \\
600 \\
600 \\
600\end{array}$ & $\begin{array}{l}481 \\
481 \\
482 \\
483\end{array}$ & $\begin{array}{l}8.3 \\
8.3 \\
7.9 \\
7.8\end{array}$ & $\begin{array}{l}28.6 \\
28.6 \\
28.4 \\
28.4\end{array}$ & $\begin{array}{l}-- \\
-- \\
--\end{array}$ & $\begin{array}{l}8.1 \\
8.1 \\
7.0 \\
6.8\end{array}$ & $\begin{array}{r}108 \\
107 \\
93 \\
89\end{array}$ \\
\hline $\begin{array}{l}22 \\
22 \\
22 \\
22\end{array}$ & $\begin{array}{l}1035 \\
1036 \\
1038 \\
1037\end{array}$ & $\begin{array}{l}0.6 \\
3.0 \\
18 \\
34\end{array}$ & $\begin{array}{l}600 \\
600 \\
600 \\
600\end{array}$ & $\begin{array}{l}518 \\
520 \\
517 \\
517\end{array}$ & $\begin{array}{l}7.5 \\
7.5 \\
7.5 \\
7.5\end{array}$ & $\begin{array}{l}24.0 \\
24.1 \\
24.1 \\
24.1\end{array}$ & $\begin{array}{l}-- \\
-- \\
--\end{array}$ & $\begin{array}{l}7.5 \\
7.5 \\
7.5 \\
7.5\end{array}$ & $\begin{array}{l}90 \\
91 \\
91 \\
91\end{array}$ \\
\hline $\begin{array}{c}\text { octob } \\
03 \\
03 \\
03 \\
03\end{array}$ & $\begin{array}{l}\text { er } \\
1056 \\
1056 \\
1057 \\
1057\end{array}$ & $\begin{array}{l}0.2 \\
2.8 \\
11 \\
24\end{array}$ & $\begin{array}{l}600 \\
600 \\
600 \\
600\end{array}$ & $\begin{array}{l}505 \\
505 \\
503 \\
505\end{array}$ & $\begin{array}{l}7.5 \\
7.5 \\
7.5 \\
7.5\end{array}$ & $\begin{array}{l}23.1 \\
23.1 \\
23.0 \\
23.0\end{array}$ & $\begin{array}{l}-- \\
- \\
-\end{array}$ & $\begin{array}{l}7.6 \\
7.5 \\
7.5 \\
7.5\end{array}$ & $\begin{array}{l}91 \\
90 \\
90 \\
90\end{array}$ \\
\hline $\begin{array}{l}17 \\
17 \\
17 \\
17\end{array}$ & $\begin{array}{l}1111 \\
1112 \\
1113 \\
1112\end{array}$ & $\begin{array}{r}0.9 \\
3.1 \\
12 \\
23\end{array}$ & $\begin{array}{l}600 \\
600 \\
600 \\
600\end{array}$ & $\begin{array}{l}526 \\
527 \\
528 \\
522\end{array}$ & $\begin{array}{l}7.4 \\
7.4 \\
7.4 \\
7.4\end{array}$ & $\begin{array}{l}19.4 \\
19.4 \\
19.4 \\
19.4\end{array}$ & $\begin{array}{l}- \\
-- \\
--\end{array}$ & $\begin{array}{l}8.2 \\
8.2 \\
8.2 \\
8.1\end{array}$ & $\begin{array}{l}90 \\
90 \\
89 \\
89\end{array}$ \\
\hline
\end{tabular}


Table 18. Water-quality data for station 401542080371801 , Ohio River at river mile 75.0, June to October, 1995.

[ft $=$ feet $; \mu \mathrm{s} / \mathrm{cm}=$ microsiemens per centimeter; ${ }^{\circ} \mathrm{C}=$ degrees celsius; $\mathrm{mg} / \mathrm{L}=$ milligrams per liter; $\cdots$ = data not collected]

\begin{tabular}{|c|c|c|c|c|c|c|c|c|c|}
\hline Date & Time & $\begin{array}{l}\text { Sampling } \\
\text { depth } \\
\text { (ft) }\end{array}$ & $\begin{array}{c}\text { Sample } \\
\text { location } \\
\text { (ft from } \\
\text { left bank) }\end{array}$ & $\begin{array}{l}\text { Specific } \\
\text { conduct- } \\
\text { ance } \\
(\mu \mathrm{s} / \mathrm{cm})\end{array}$ & $\begin{array}{l}\mathrm{pH} \\
\text { (stan. } \\
\text { dard } \\
\text { units) }\end{array}$ & $\begin{array}{c}\text { Temper: } \\
\text { ature, } \\
\text { water } \\
\left({ }^{\circ} \mathrm{C}\right)\end{array}$ & $\begin{array}{l}\text { Trans- } \\
\text { parency } \\
\text { (Secchi } \\
\text { disk) } \\
\text { (ft) }\end{array}$ & $\begin{array}{l}\text { Dissolved } \\
\text { oxygen } \\
\text { (mg/L) }\end{array}$ & $\begin{array}{c}\text { Dissolved } \\
\text { oxygen } \\
\text { (percent } \\
\text { satura- } \\
\text { tion) }\end{array}$ \\
\hline $\begin{array}{r}\text { July } \\
11 \\
11 \\
11 \\
11\end{array}$ & $\begin{array}{l}1550 \\
1550 \\
1552 \\
1551\end{array}$ & $\begin{array}{l}0.6 \\
3.1 \\
14 \\
29\end{array}$ & $\begin{array}{l}600 \\
600 \\
600 \\
600\end{array}$ & $\begin{array}{l}421 \\
422 \\
424 \\
429\end{array}$ & $\begin{array}{l}7.9 \\
7.9 \\
7.4 \\
7.4\end{array}$ & $\begin{array}{l}28.1 \\
27.9 \\
26.5 \\
26.4\end{array}$ & $\begin{array}{l}\cdots \\
\cdots \\
\cdots\end{array}$ & $\begin{array}{l}9.1 \\
9.1 \\
7.4 \\
7.4\end{array}$ & $\begin{array}{r}119 \\
118 \\
94 \\
94\end{array}$ \\
\hline $\begin{array}{l}25 \\
25 \\
25 \\
25\end{array}$ & $\begin{array}{l}1646 \\
1647 \\
1650 \\
1648\end{array}$ & $\begin{array}{l}0.3 \\
3.1 \\
18 \\
35\end{array}$ & $\begin{array}{l}600 \\
600 \\
600 \\
600\end{array}$ & $\begin{array}{l}426 \\
427 \\
423 \\
428\end{array}$ & $\begin{array}{l}7.8 \\
7.7 \\
7.5 \\
7.5\end{array}$ & $\begin{array}{l}29.6 \\
29.5 \\
28.8 \\
28.6\end{array}$ & $\begin{array}{l}\cdots \\
\cdots \\
\cdots\end{array}$ & $\begin{array}{l}7.8 \\
7.5 \\
6.5 \\
6.1\end{array}$ & $\begin{array}{r}105 \\
101 \\
86 \\
81\end{array}$ \\
\hline $\begin{array}{c}\text { Augus } \\
08 \\
08 \\
08 \\
08\end{array}$ & $\begin{array}{l}t \\
1408 \\
1409 \\
1410 \\
1409\end{array}$ & $\begin{array}{l}0.3 \\
3.3 \\
14 \\
28\end{array}$ & $\begin{array}{l}600 \\
600 \\
600 \\
600\end{array}$ & $\begin{array}{l}466 \\
468 \\
468 \\
471\end{array}$ & $\begin{array}{l}7.6 \\
7.5 \\
7.5 \\
7.5\end{array}$ & $\begin{array}{l}28.8 \\
28.8 \\
28.8 \\
28.8\end{array}$ & $\begin{array}{l}-- \\
- \\
\cdots\end{array}$ & $\begin{array}{l}7.1 \\
6.9 \\
6.7 \\
6.8\end{array}$ & $\begin{array}{l}93 \\
91 \\
88 \\
89\end{array}$ \\
\hline $\begin{array}{l}22 \\
22 \\
22 \\
22\end{array}$ & $\begin{array}{l}1546 \\
1546 \\
1548 \\
1547\end{array}$ & $\begin{array}{l}0.3 \\
3.3 \\
14 \\
29\end{array}$ & $\begin{array}{l}600 \\
600 \\
600 \\
600\end{array}$ & $\begin{array}{l}525 \\
525 \\
527 \\
525\end{array}$ & $\begin{array}{l}8.0 \\
8.0 \\
8.0 \\
7.9\end{array}$ & $\begin{array}{l}30.6 \\
30.6 \\
30.6 \\
30.6\end{array}$ & $\begin{array}{l}-- \\
-- \\
--\end{array}$ & $\begin{array}{l}7.8 \\
7.9 \\
7.8 \\
7.5\end{array}$ & $\begin{array}{l}105 \\
107 \\
106 \\
102\end{array}$ \\
\hline $\begin{array}{r}\text { Septe } \\
07 \\
07 \\
07 \\
07\end{array}$ & $\begin{array}{l}\text { mber } \\
1346 \\
1347 \\
1349 \\
1348\end{array}$ & $\begin{array}{r}0.2 \\
3.0 \\
14 \\
28\end{array}$ & $\begin{array}{l}600 \\
600 \\
600 \\
600\end{array}$ & $\begin{array}{l}483 \\
482 \\
484 \\
485\end{array}$ & $\begin{array}{l}8.3 \\
8.3 \\
7.9 \\
7.9\end{array}$ & $\begin{array}{l}28.4 \\
28.3 \\
28.1 \\
28.0\end{array}$ & $\begin{array}{l}-- \\
- \\
-\end{array}$ & $\begin{array}{l}8.2 \\
8.2 \\
7.1 \\
7.0\end{array}$ & $\begin{array}{r}108 \\
108 \\
93 \\
91\end{array}$ \\
\hline $\begin{array}{l}22 \\
22 \\
22 \\
22\end{array}$ & $\begin{array}{l}1027 \\
1027 \\
1029 \\
1028\end{array}$ & $\begin{array}{l}1.1 \\
3.1 \\
14 \\
28\end{array}$ & $\begin{array}{l}600 \\
600 \\
600 \\
600\end{array}$ & $\begin{array}{l}518 \\
519 \\
519 \\
521\end{array}$ & $\begin{array}{l}7.5 \\
7.5 \\
7.5 \\
7.5\end{array}$ & $\begin{array}{l}23.7 \\
23.7 \\
23.7 \\
23.7\end{array}$ & $\begin{array}{l}-- \\
-- \\
-\end{array}$ & $\begin{array}{l}7.5 \\
7.4 \\
7.5 \\
7.5\end{array}$ & $\begin{array}{l}90 \\
90 \\
91 \\
90\end{array}$ \\
\hline $\begin{array}{c}\text { Octob } \\
03 \\
03 \\
03 \\
03\end{array}$ & $\begin{array}{l}\text { er } \\
1049 \\
1049 \\
1050 \\
1050\end{array}$ & $\begin{array}{r}0.5 \\
3.2 \\
14 \\
28\end{array}$ & $\begin{array}{l}600 \\
600 \\
600 \\
600\end{array}$ & $\begin{array}{l}501 \\
502 \\
503 \\
504\end{array}$ & $\begin{array}{l}7.5 \\
7.5 \\
7.5 \\
7.5\end{array}$ & $\begin{array}{l}22.8 \\
22.8 \\
22.8 \\
22.8\end{array}$ & $\begin{array}{l}-- \\
-- \\
--\end{array}$ & $\begin{array}{l}7.2 \\
7.3 \\
7.2 \\
7.2\end{array}$ & $\begin{array}{l}85 \\
87 \\
85 \\
86\end{array}$ \\
\hline $\begin{array}{l}17 \\
17 \\
17 \\
17\end{array}$ & $\begin{array}{l}1104 \\
1104 \\
1105 \\
1105\end{array}$ & $\begin{array}{l}0.8 \\
3.0 \\
14 \\
28\end{array}$ & $\begin{array}{l}600 \\
600 \\
600 \\
600\end{array}$ & $\begin{array}{l}525 \\
526 \\
527 \\
519\end{array}$ & $\begin{array}{l}7.5 \\
7.5 \\
7.5 \\
7.4\end{array}$ & $\begin{array}{l}19.7 \\
19.7 \\
19.6 \\
19.6\end{array}$ & $\begin{array}{l}-- \\
-- \\
--\end{array}$ & $\begin{array}{l}8.1 \\
8.1 \\
7.9 \\
7.9\end{array}$ & $\begin{array}{l}89 \\
89 \\
87 \\
87\end{array}$ \\
\hline
\end{tabular}


Table 19. Water-quality data for station 401422080391701, Ohio River at river mile 77.4, June to October, 1995.

[ft $=$ feet $; \mu \mathrm{s} / \mathrm{cm}=$ microsiemens per centimeter; ${ }^{\circ} \mathrm{C}=$ degrees Celsius; $\mathrm{mg} / \mathrm{L}=$ milligrams per liter; $\cdot-$ = data not collected]

\begin{tabular}{|c|c|c|c|c|c|c|c|c|c|}
\hline Date & Time & $\begin{array}{l}\text { Sampling } \\
\text { depth } \\
\text { (ft) }\end{array}$ & $\begin{array}{c}\text { Sample } \\
\text { location } \\
\text { (ft from } \\
\text { left bank) }\end{array}$ & $\begin{array}{l}\text { Specific } \\
\text { conduct - } \\
\text { ance } \\
(\mu \mathrm{s} / \mathrm{cm})\end{array}$ & $\begin{array}{l}\mathrm{pH} \\
\text { (stan- } \\
\text { dard } \\
\text { units) }\end{array}$ & $\begin{array}{c}\text { Temper- } \\
\text { ature, } \\
\text { water } \\
\left({ }^{\circ} \mathrm{C}\right)\end{array}$ & $\begin{array}{l}\text { Trans- } \\
\text { parency } \\
\text { (secchi } \\
\text { disk) } \\
\text { (ft) }\end{array}$ & $\begin{array}{l}\text { Dissolved } \\
\text { oxygen } \\
(\mathrm{mg} / \mathrm{L})\end{array}$ & $\begin{array}{c}\text { Dissolved } \\
\text { oxygen } \\
\text { (percent } \\
\text { satura- } \\
\text { tion) }\end{array}$ \\
\hline
\end{tabular}

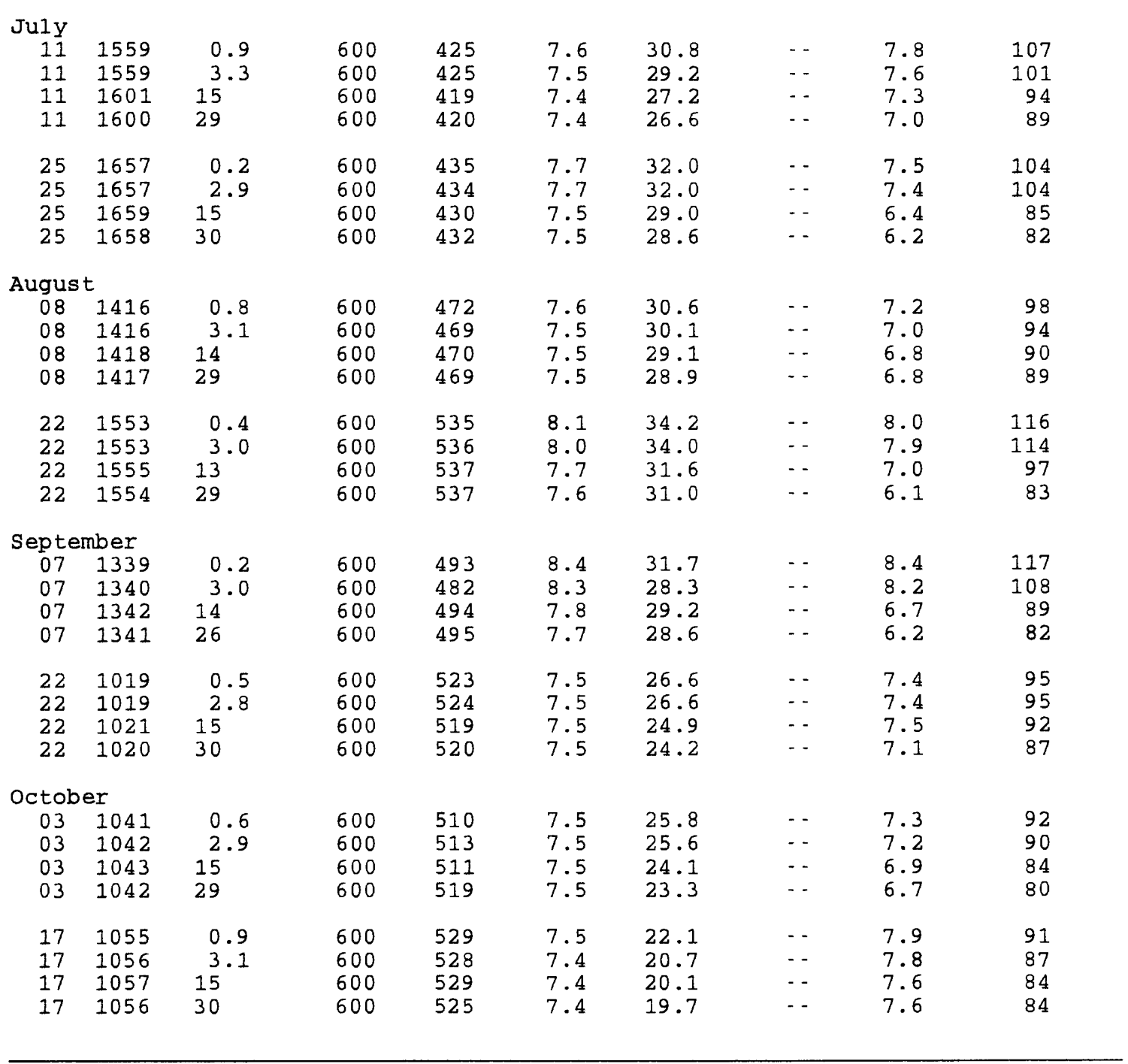


Table 20. Water-quality data for station 401148080400901, Ohio River at river mile 80.5, June to October, 1995.

[ft = feet; $\mu \mathrm{s} / \mathrm{cm}=$ microsiemens per centimeter $;{ }^{\circ} \mathrm{C}=$ degrees Celsius; $\mathrm{mg} / \mathrm{L}=\mathrm{milligrams}$ per liter; $\cdots$ = data not collected]

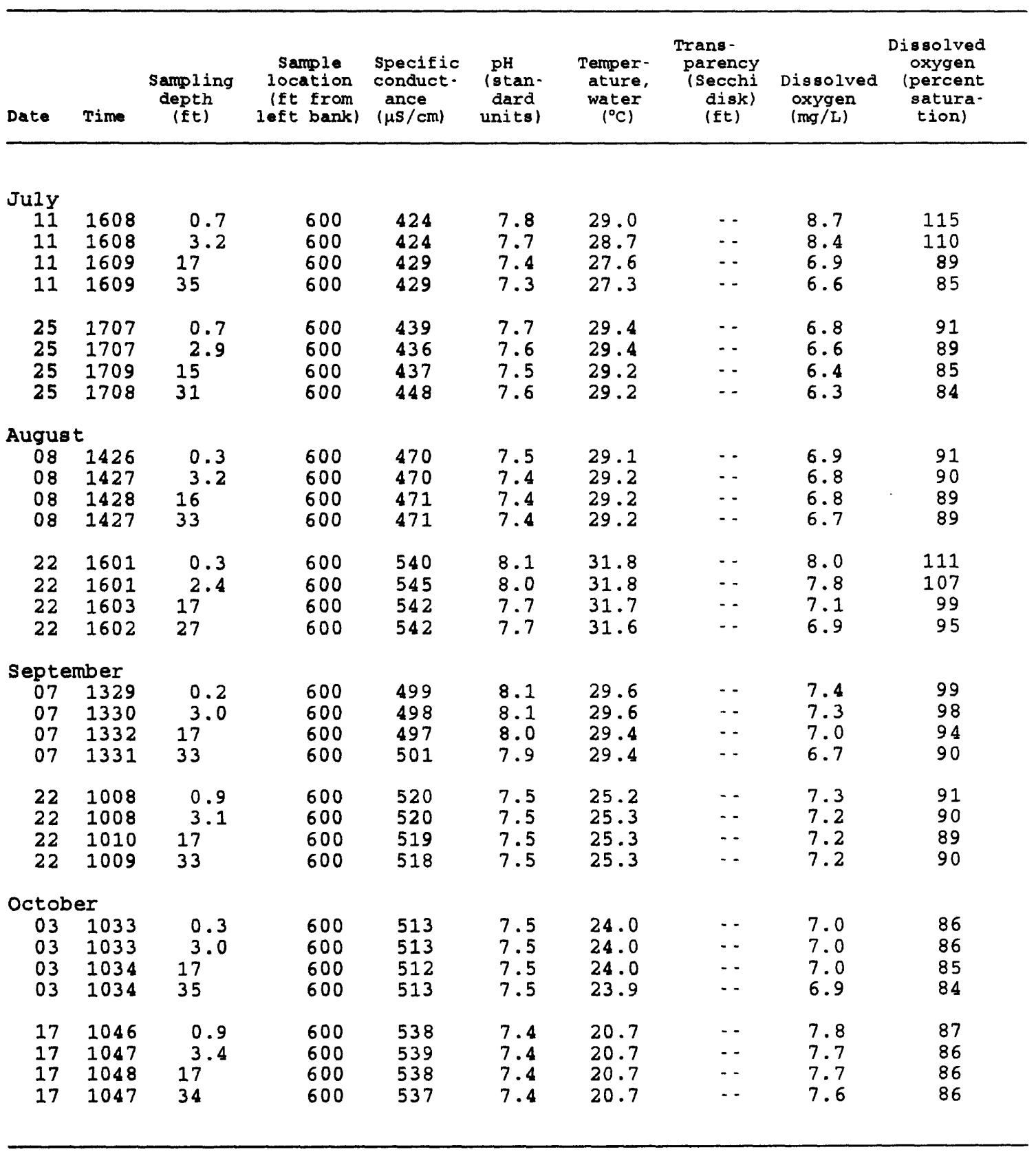


Table 21. Water-quality data for station 401031080411601, Ohio River at river mile 82.3, June to October, 1995.

\begin{tabular}{|c|c|c|c|c|c|c|c|c|c|}
\hline Date & Time & $\begin{array}{l}\text { Sampling } \\
\text { depth } \\
\text { (ft) }\end{array}$ & $\begin{array}{c}\text { Sample } \\
\text { location } \\
\text { (ft from } \\
\text { left bank) }\end{array}$ & $\begin{array}{l}\text { Specific } \\
\text { conduct- } \\
\text { ance } \\
(\mu S / \mathrm{cm})\end{array}$ & $\begin{array}{l}\text { pH } \\
\text { (stan- } \\
\text { dard } \\
\text { units) }\end{array}$ & $\begin{array}{c}\text { Temper- } \\
\text { ature, } \\
\text { water } \\
\left({ }^{\circ} \mathrm{C}\right)\end{array}$ & $\begin{array}{l}\text { Trans- } \\
\text { parency } \\
\text { (Secchi } \\
\text { disk) } \\
\text { (ft) }\end{array}$ & $\begin{array}{l}\text { Dissolved } \\
\text { oxygen } \\
(\mathrm{mg} / \mathrm{L})\end{array}$ & $\begin{array}{c}\text { Dissolved } \\
\text { oxygen } \\
\text { (percent } \\
\text { satura- } \\
\text { tion) }\end{array}$ \\
\hline $\begin{array}{r}\text { July } \\
11 \\
11 \\
11 \\
11\end{array}$ & $\begin{array}{l}1614 \\
1615 \\
1617 \\
1615\end{array}$ & $\begin{array}{l}0.5 \\
3.1 \\
15 \\
30\end{array}$ & $\begin{array}{l}800 \\
800 \\
800 \\
800\end{array}$ & $\begin{array}{l}425 \\
425 \\
433 \\
451\end{array}$ & $\begin{array}{l}8.3 \\
8.0 \\
7.4 \\
7.3\end{array}$ & $\begin{array}{l}29.7 \\
28.8 \\
27.3 \\
27.0\end{array}$ & $\begin{array}{l}-- \\
\cdots \\
\cdots\end{array}$ & $\begin{array}{l}9.7 \\
9.2 \\
7.1 \\
6.6\end{array}$ & $\begin{array}{r}131 \\
122 \\
92 \\
85\end{array}$ \\
\hline $\begin{array}{l}25 \\
25 \\
25 \\
25\end{array}$ & $\begin{array}{l}1713 \\
1714 \\
1716 \\
1715\end{array}$ & $\begin{array}{l}0.3 \\
2.5 \\
12 \\
22\end{array}$ & $\begin{array}{l}800 \\
800 \\
800 \\
800\end{array}$ & $\begin{array}{l}450 \\
451 \\
448 \\
457\end{array}$ & $\begin{array}{l}7.9 \\
8.0 \\
7.9 \\
7.6\end{array}$ & $\begin{array}{l}29.6 \\
29.6 \\
29.5 \\
28.8\end{array}$ & $\begin{array}{l}-- \\
\cdots \\
\cdots\end{array}$ & $\begin{array}{l}8.1 \\
8.2 \\
8.0 \\
6.4\end{array}$ & $\begin{array}{r}108 \\
110 \\
108 \\
84\end{array}$ \\
\hline $\begin{array}{c}\text { Augus } \\
08 \\
08 \\
08 \\
08\end{array}$ & $\begin{array}{l}\text { t } \\
1432 \\
1432 \\
1434 \\
1433\end{array}$ & $\begin{array}{l}0.4 \\
3.2 \\
15 \\
30\end{array}$ & $\begin{array}{l}800 \\
800 \\
800 \\
800\end{array}$ & $\begin{array}{l}477 \\
477 \\
477 \\
477\end{array}$ & $\begin{array}{l}7.5 \\
7.5 \\
7.5 \\
7.5\end{array}$ & $\begin{array}{l}29.4 \\
29.4 \\
29.4 \\
29.4\end{array}$ & $\begin{array}{l}- \\
\cdots \\
\cdots \\
\cdots\end{array}$ & $\begin{array}{l}6.9 \\
6.8 \\
6.8 \\
6.8\end{array}$ & $\begin{array}{l}92 \\
91 \\
91 \\
90\end{array}$ \\
\hline $\begin{array}{l}22 \\
22 \\
22 \\
22\end{array}$ & $\begin{array}{l}1641 \\
1641 \\
1643 \\
1642\end{array}$ & $\begin{array}{l}0.7 \\
3.1 \\
24 \\
38\end{array}$ & $\begin{array}{l}800 \\
800 \\
800 \\
800\end{array}$ & $\begin{array}{l}552 \\
552 \\
560 \\
560\end{array}$ & $\begin{array}{l}8.3 \\
8.2 \\
7.9 \\
7.7\end{array}$ & $\begin{array}{l}31.7 \\
31.7 \\
31.4 \\
31.2\end{array}$ & $\begin{array}{l}\cdots \\
\cdots \\
\cdots\end{array}$ & $\begin{array}{l}8.4 \\
8.3 \\
7.6 \\
7.0\end{array}$ & $\begin{array}{r}116 \\
115 \\
104 \\
97\end{array}$ \\
\hline $\begin{array}{c}\text { Septe } \\
07 \\
07 \\
07 \\
07\end{array}$ & $\begin{array}{l}\text { mber } \\
1317 \\
1318 \\
1320 \\
1319\end{array}$ & $\begin{array}{l}0.2 \\
3.0 \\
13 \\
26\end{array}$ & $\begin{array}{l}800 \\
800 \\
800 \\
800\end{array}$ & $\begin{array}{l}508 \\
509 \\
509 \\
509\end{array}$ & $\begin{array}{l}8.1 \\
8.2 \\
8.0 \\
7.9\end{array}$ & $\begin{array}{l}29.4 \\
29.4 \\
29.4 \\
29.3\end{array}$ & $\begin{array}{l}\cdots \\
\cdots \\
\cdots\end{array}$ & $\begin{array}{l}7.4 \\
7.4 \\
7.1 \\
6.8\end{array}$ & $\begin{array}{r}100 \\
100 \\
96 \\
91\end{array}$ \\
\hline $\begin{array}{l}22 \\
22 \\
22 \\
22\end{array}$ & $\begin{array}{l}1000 \\
1000 \\
1002 \\
1001\end{array}$ & $\begin{array}{l}0.7 \\
3.0 \\
15 \\
30\end{array}$ & $\begin{array}{l}800 \\
800 \\
800 \\
800\end{array}$ & $\begin{array}{l}525 \\
532 \\
518 \\
520\end{array}$ & $\begin{array}{l}7.5 \\
7.5 \\
7.5 \\
7.5\end{array}$ & $\begin{array}{l}24.9 \\
24.9 \\
25.0 \\
25.0\end{array}$ & $\begin{array}{l}\cdots \\
\cdots \\
-\end{array}$ & $\begin{array}{l}7.5 \\
7.5 \\
7.5 \\
7.4\end{array}$ & $\begin{array}{l}92 \\
92 \\
92 \\
92\end{array}$ \\
\hline $\begin{array}{c}\text { octob } \\
03 \\
03 \\
03 \\
03\end{array}$ & $\begin{array}{l}\text { er } \\
1028 \\
1028 \\
1029 \\
1029\end{array}$ & $\begin{array}{l}0.2 \\
2.8 \\
22 \\
44\end{array}$ & $\begin{array}{l}800 \\
800 \\
800 \\
800\end{array}$ & $\begin{array}{l}520 \\
519 \\
517 \\
512\end{array}$ & $\begin{array}{l}7.5 \\
7.5 \\
7.5 \\
7.5\end{array}$ & $\begin{array}{l}23.4 \\
23.4 \\
23.4 \\
23.4\end{array}$ & $\begin{array}{l}- \\
\cdots \\
\cdots \\
-\end{array}$ & $\begin{array}{l}7.1 \\
7.0 \\
6.8 \\
6.9\end{array}$ & $\begin{array}{l}86 \\
84 \\
82 \\
83\end{array}$ \\
\hline $\begin{array}{l}17 \\
17 \\
17 \\
17\end{array}$ & $\begin{array}{l}1040 \\
1041 \\
1042 \\
1041\end{array}$ & $\begin{array}{l}0.5 \\
3.3 \\
15 \\
30\end{array}$ & $\begin{array}{l}800 \\
800 \\
800 \\
800\end{array}$ & $\begin{array}{l}544 \\
543 \\
544 \\
548\end{array}$ & $\begin{array}{l}7.5 \\
7.4 \\
7.4 \\
7.4\end{array}$ & $\begin{array}{l}20.4 \\
20.5 \\
20.4 \\
20.4\end{array}$ & $\begin{array}{l}- \\
-- \\
-\end{array}$ & $\begin{array}{l}7.8 \\
7.8 \\
7.6 \\
7.6\end{array}$ & $\begin{array}{l}87 \\
87 \\
85 \\
85\end{array}$ \\
\hline
\end{tabular}


Table 22. Water-quality data for station 400913080421201, Ohio River at river mile 84.0, June to October, 1995.

[ft $=$ feet $; \mu \mathrm{s} / \mathrm{cm}=$ microsiemens per centimeter $;{ }^{\circ} \mathrm{C}=$ degrees Celsius; $\mathrm{mg} / \mathrm{L}=$ milligrams per liter; $\cdots=$ data not collected]

\begin{tabular}{|c|c|c|c|c|c|c|c|c|c|}
\hline Date & Time & $\begin{array}{l}\text { Sampling } \\
\text { depth } \\
\text { (ft) }\end{array}$ & $\begin{array}{c}\text { Sample } \\
\text { location } \\
\text { (ft from } \\
\text { left bank) }\end{array}$ & $\begin{array}{l}\text { Specific } \\
\text { conduct- } \\
\text { ance } \\
(\mu \mathrm{s} / \mathrm{cm})\end{array}$ & $\begin{array}{l}\mathrm{pH} \\
\text { (stan- } \\
\text { dard } \\
\text { units) }\end{array}$ & $\begin{array}{c}\text { Temper- } \\
\text { ature, } \\
\text { water } \\
\left({ }^{\circ} \mathrm{C}\right)\end{array}$ & $\begin{array}{l}\text { Trans - } \\
\text { parency } \\
\text { (secchi } \\
\text { disk) } \\
\text { (ft) }\end{array}$ & $\begin{array}{c}\text { Dissolved } \\
\text { oxygen } \\
(\mathrm{mg} / \mathrm{L})\end{array}$ & $\begin{array}{c}\text { Dissolved } \\
\text { oxygen } \\
\text { (percent } \\
\text { satura. } \\
\text { tion) }\end{array}$ \\
\hline
\end{tabular}

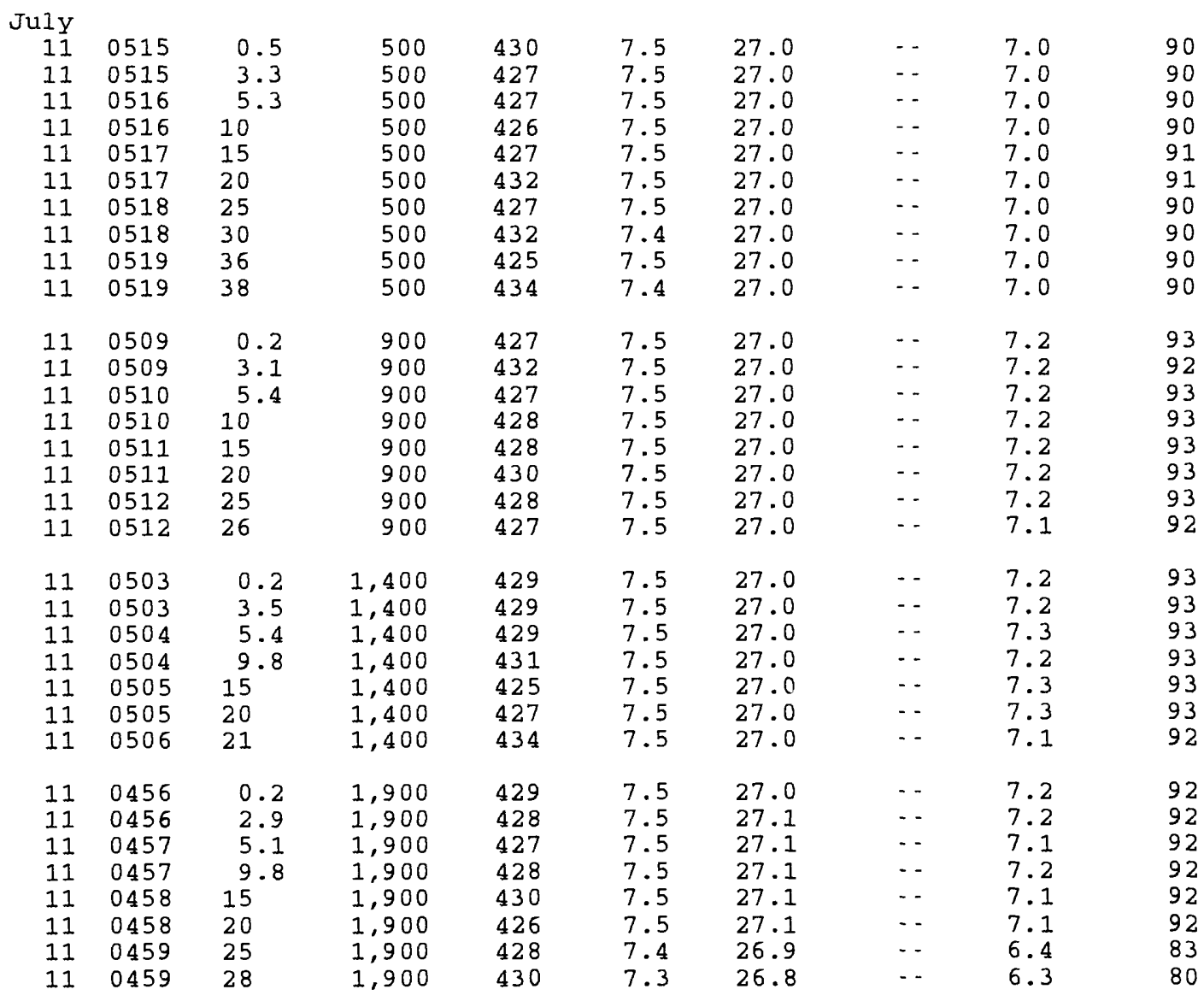


Table 22. Water-quality data for station 400913080421201, Ohio River at river mile 84.0, June to October, 1995, Continued.

[ft $=$ feet $; \mu \mathrm{S} / \mathrm{cm}=$ microsiemens per centimeter $;{ }^{\circ} \mathrm{C}=$ degrees Celsius; $\mathrm{mg} / \mathrm{L}=$ mililigrams per liter; $\cdot-$ = data not collected]

\begin{tabular}{|c|c|c|c|c|c|c|c|c|c|}
\hline Date & Time & $\begin{array}{l}\text { Sampling } \\
\text { depth } \\
\text { (ft) }\end{array}$ & $\begin{array}{c}\text { Sample } \\
\text { location } \\
\text { (ft from } \\
\text { left bank) }\end{array}$ & $\begin{array}{l}\text { Specific } \\
\text { conduct- } \\
\text { ance } \\
(\mu S / \mathrm{cm})\end{array}$ & $\begin{array}{l}\text { pH } \\
\text { (stan- } \\
\text { dard } \\
\text { units) }\end{array}$ & $\begin{array}{c}\text { Temper- } \\
\text { ature, } \\
\text { water } \\
\left({ }^{\circ} \mathrm{C}\right)\end{array}$ & $\begin{array}{c}\text { Trans - } \\
\text { parency } \\
\text { (secchi } \\
\text { disk) } \\
\text { (ft) }\end{array}$ & $\begin{array}{l}\text { Dissolved } \\
\text { oxygen } \\
\text { (mg/L) }\end{array}$ & $\begin{array}{c}\text { Dissolved } \\
\text { oxygen } \\
\text { (percent } \\
\text { satura- } \\
\text { tion) }\end{array}$ \\
\hline
\end{tabular}

\begin{tabular}{|c|c|c|c|c|c|c|c|c|c|}
\hline $\begin{array}{r}\text { July } \\
11 \\
11 \\
11 \\
11 \\
11 \\
11 \\
11 \\
11 \\
11 \\
11 \\
11\end{array}$ & $\begin{array}{l}1649 \\
1649 \\
1650 \\
1651 \\
1651 \\
1652 \\
1652 \\
1653 \\
1654 \\
1654 \\
1655\end{array}$ & $\begin{array}{l}0.5 \\
3.1 \\
5.0 \\
9.9 \\
15 \\
20 \\
25 \\
30 \\
35 \\
40 \\
45\end{array}$ & $\begin{array}{l}500 \\
500 \\
500 \\
500 \\
500 \\
500 \\
500 \\
500 \\
500 \\
500 \\
500\end{array}$ & $\begin{array}{l}443 \\
442 \\
443 \\
441 \\
444 \\
444 \\
443 \\
441 \\
442 \\
445 \\
444\end{array}$ & $\begin{array}{l}7.6 \\
7.5 \\
7.5 \\
7.4 \\
7.4 \\
7.4 \\
7.3 \\
7.3 \\
7.3 \\
7.3 \\
7.3\end{array}$ & $\begin{array}{l}27.9 \\
27.5 \\
27.6 \\
27.4 \\
27.1 \\
27.1 \\
27.1 \\
27.1 \\
27.0 \\
27.0 \\
27.0\end{array}$ & $\begin{array}{l}- \\
- \\
- \\
- \\
-- \\
-- \\
- \\
- \\
--\end{array}$ & $\begin{array}{l}8.0 \\
7.3 \\
7.5 \\
7.3 \\
6.9 \\
6.9 \\
6.7 \\
6.7 \\
6.5 \\
6.4 \\
6.4\end{array}$ & $\begin{array}{r}105 \\
95 \\
97 \\
94 \\
88 \\
88 \\
86 \\
86 \\
83 \\
83 \\
82\end{array}$ \\
\hline $\begin{array}{l}11 \\
11 \\
11 \\
11 \\
11 \\
11 \\
11 \\
11\end{array}$ & $\begin{array}{l}1639 \\
1640 \\
1640 \\
1641 \\
1642 \\
1643 \\
1643 \\
1644\end{array}$ & $\begin{array}{l}0.4 \\
3.2 \\
5.1 \\
9.9 \\
15 \\
20 \\
25 \\
29\end{array}$ & $\begin{array}{l}900 \\
900 \\
900 \\
900 \\
900 \\
900 \\
900 \\
900\end{array}$ & $\begin{array}{l}441 \\
443 \\
441 \\
445 \\
444 \\
443 \\
444 \\
442\end{array}$ & $\begin{array}{l}8.1 \\
8.0 \\
7.5 \\
7.4 \\
7.4 \\
7.4 \\
7.3 \\
7.3\end{array}$ & $\begin{array}{l}29.0 \\
28.7 \\
27.6 \\
27.2 \\
27.2 \\
27.1 \\
27.0 \\
27.0\end{array}$ & $\begin{array}{l}- \\
\cdots \\
- \\
\cdots \\
\cdots \\
\cdots\end{array}$ & $\begin{array}{l}9.5 \\
8.6 \\
7.5 \\
6.9 \\
7.0 \\
6.8 \\
6.6 \\
6.4\end{array}$ & $\begin{array}{r}126 \\
114 \\
97 \\
89 \\
90 \\
88 \\
84 \\
82\end{array}$ \\
\hline $\begin{array}{l}11 \\
11 \\
11 \\
11 \\
11 \\
11 \\
11\end{array}$ & $\begin{array}{l}1632 \\
1632 \\
1633 \\
1634 \\
1634 \\
1635 \\
1636\end{array}$ & $\begin{array}{l}0.3 \\
3.1 \\
5.0 \\
10 \\
15 \\
20 \\
22\end{array}$ & $\begin{array}{l}1,400 \\
1,400 \\
1,400 \\
1,400 \\
1,400 \\
1,400 \\
1,400\end{array}$ & $\begin{array}{l}441 \\
445 \\
442 \\
442 \\
444 \\
446 \\
444\end{array}$ & $\begin{array}{l}8.1 \\
7.8 \\
7.6 \\
7.4 \\
7.4 \\
7.3 \\
7.3\end{array}$ & $\begin{array}{l}29.0 \\
28.3 \\
27.9 \\
27.2 \\
27.0 \\
27.0 \\
27.0\end{array}$ & $\begin{array}{l}-- \\
-- \\
\cdots \\
-- \\
\cdots \\
-\end{array}$ & $\begin{array}{l}9.4 \\
8.4 \\
7.8 \\
7.1 \\
6.8 \\
6.7 \\
6.5\end{array}$ & $\begin{array}{r}125 \\
111 \\
101 \\
91 \\
87 \\
86 \\
83\end{array}$ \\
\hline $\begin{array}{l}11 \\
11 \\
11 \\
11 \\
11 \\
11 \\
11 \\
11\end{array}$ & $\begin{array}{l}1624 \\
1624 \\
1625 \\
1626 \\
1627 \\
1627 \\
1628 \\
1629\end{array}$ & $\begin{array}{l}0.4 \\
3.0 \\
5.0 \\
10 \\
15 \\
20 \\
25 \\
28\end{array}$ & $\begin{array}{l}1,900 \\
1,900 \\
1,900 \\
1,900 \\
1,9000 \\
1,900 \\
1,900 \\
1,900\end{array}$ & $\begin{array}{l}440 \\
443 \\
443 \\
443 \\
443 \\
443 \\
441 \\
439\end{array}$ & $\begin{array}{l}8.1 \\
8.0 \\
7.6 \\
7.4 \\
7.4 \\
7.4 \\
7.4 \\
7.2\end{array}$ & $\begin{array}{l}28.9 \\
28.4 \\
27.7 \\
27.4 \\
27.1 \\
27.1 \\
27.0 \\
26.9\end{array}$ & $\begin{array}{l}-- \\
-- \\
-- \\
-- \\
-- \\
-- \\
-\end{array}$ & $\begin{array}{l}9.4 \\
9.0 \\
8.0 \\
7.1 \\
6.9 \\
6.9 \\
6.8 \\
5.5\end{array}$ & $\begin{array}{r}124 \\
119 \\
104 \\
92 \\
89 \\
88 \\
87 \\
71\end{array}$ \\
\hline
\end{tabular}


Table 22. Water-quality data for station 400913080421201, Ohio River at river mile 84.0, June to October, 1995, Continued.

[ft $=$ feet $; \mu \mathrm{s} / \mathrm{cm}=$ microsiemens per centimeter; ${ }^{\circ} \mathrm{C}=$ degrees Celsius; $\mathrm{mg} / \mathrm{L}=\mathrm{milligrams}$ per liter;.. = data not collected]

\begin{tabular}{|c|c|c|c|c|c|c|c|c|c|}
\hline Date & Time & $\begin{array}{l}\text { Sampling } \\
\text { depth } \\
\text { (ft) }\end{array}$ & $\begin{array}{l}\text { Sample } \\
\text { location } \\
\text { (ft from } \\
\text { left bank) }\end{array}$ & $\begin{array}{l}\text { Specific } \\
\text { conduct- } \\
\text { ance } \\
(\mu s / \mathrm{cm})\end{array}$ & $\begin{array}{l}\mathrm{pH} \\
\text { (stan- } \\
\text { dard } \\
\text { units) }\end{array}$ & $\begin{array}{c}\text { Temper- } \\
\text { ature, } \\
\text { water } \\
\left({ }^{\circ} \mathrm{C}\right)\end{array}$ & $\begin{array}{l}\text { Trans- } \\
\text { parency } \\
\text { (secchi } \\
\text { disk) } \\
\text { (ft) }\end{array}$ & $\begin{array}{l}\text { Dissolved } \\
\text { oxygen } \\
(\mathrm{mg} / \mathrm{L})\end{array}$ & $\begin{array}{c}\text { Dissolved } \\
\text { oxygen } \\
\text { (percent } \\
\text { satura- } \\
\text { tion) }\end{array}$ \\
\hline
\end{tabular}

\begin{tabular}{|c|c|c|c|c|c|c|c|c|c|}
\hline $\begin{array}{r}\text { July } \\
25 \\
25 \\
25 \\
25 \\
25 \\
25 \\
25 \\
25 \\
25 \\
25 \\
25\end{array}$ & $\begin{array}{l}1749 \\
1749 \\
1750 \\
1750 \\
1751 \\
1751 \\
1752 \\
1752 \\
1753 \\
1753 \\
1754\end{array}$ & $\begin{array}{c}0.4 \\
3.1 \\
5.1 \\
10 \\
15 \\
20 \\
25 \\
30 \\
35 \\
40 \\
44\end{array}$ & $\begin{array}{l}500 \\
500 \\
500 \\
500 \\
500 \\
500 \\
500 \\
500 \\
500 \\
500 \\
500\end{array}$ & $\begin{array}{l}478 \\
479 \\
480 \\
476 \\
475 \\
469 \\
468 \\
465 \\
474 \\
471 \\
472\end{array}$ & $\begin{array}{l}8.4 \\
8.4 \\
8.4 \\
8.0 \\
8.0 \\
7.8 \\
7.7 \\
7.7 \\
7.9 \\
7.7 \\
7.7\end{array}$ & $\begin{array}{l}29.7 \\
29.7 \\
29.7 \\
29.2 \\
29.3 \\
28.9 \\
28.8 \\
28.8 \\
28.8 \\
28.8 \\
28.8\end{array}$ & $\begin{array}{l}-- \\
-- \\
-- \\
-- \\
-- \\
-- \\
-- \\
-- \\
-- \\
--\end{array}$ & $\begin{array}{l}9.0 \\
9.0 \\
8.9 \\
7.4 \\
7.8 \\
6.7 \\
6.4 \\
6.3 \\
6.3 \\
6.2 \\
6.2\end{array}$ & $\begin{array}{r}121 \\
122 \\
120 \\
99 \\
104 \\
88 \\
84 \\
83 \\
83 \\
83 \\
82\end{array}$ \\
\hline $\begin{array}{l}25 \\
25 \\
25 \\
25 \\
25 \\
25 \\
25 \\
25\end{array}$ & $\begin{array}{l}1741 \\
1741 \\
1742 \\
1742 \\
1743 \\
1743 \\
1744 \\
1744\end{array}$ & $\begin{array}{l}0.2 \\
3.0 \\
5.1 \\
9.9 \\
15 \\
20 \\
25 \\
26\end{array}$ & 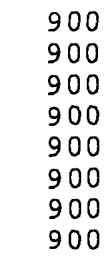 & $\begin{array}{l}478 \\
477 \\
475 \\
477 \\
476 \\
473 \\
471 \\
470\end{array}$ & $\begin{array}{l}8.2 \\
8.2 \\
8.1 \\
8.1 \\
8.0 \\
7.9 \\
7.7 \\
7.7\end{array}$ & $\begin{array}{l}29.6 \\
29.5 \\
29.4 \\
29.4 \\
29.3 \\
29.2 \\
28.8 \\
28.8\end{array}$ & $\begin{array}{l}-- \\
-- \\
-- \\
-- \\
-- \\
- \\
--\end{array}$ & $\begin{array}{l}8.5 \\
8.5 \\
8.2 \\
8.0 \\
7.8 \\
7.5 \\
6.3 \\
6.2\end{array}$ & $\begin{array}{r}114 \\
114 \\
110 \\
107 \\
104 \\
100 \\
83 \\
83\end{array}$ \\
\hline $\begin{array}{l}25 \\
25 \\
25 \\
25 \\
25 \\
25 \\
25\end{array}$ & $\begin{array}{l}1732 \\
1732 \\
1733 \\
1733 \\
1734 \\
1734 \\
1735\end{array}$ & $\begin{array}{l}0.5 \\
3.0 \\
4.9 \\
10 \\
15 \\
20 \\
21\end{array}$ & $\begin{array}{l}1,400 \\
1,400 \\
1,400 \\
1,400 \\
1,400 \\
1,400 \\
1,400\end{array}$ & $\begin{array}{l}476 \\
477 \\
477 \\
479 \\
473 \\
476 \\
476\end{array}$ & $\begin{array}{l}8.0 \\
8.0 \\
8.0 \\
8.0 \\
7.8 \\
7.7 \\
7.7\end{array}$ & $\begin{array}{l}29.3 \\
29.3 \\
29.3 \\
29.3 \\
29.0 \\
28.8 \\
28.8\end{array}$ & $\begin{array}{l}- \\
- \\
- \\
- \\
\cdots \\
- \\
-\end{array}$ & $\begin{array}{l}7.9 \\
7.9 \\
7.9 \\
7.9 \\
6.6 \\
6.2 \\
6.2\end{array}$ & $\begin{array}{r}106 \\
106 \\
105 \\
105 \\
88 \\
82 \\
82\end{array}$ \\
\hline $\begin{array}{l}25 \\
25 \\
25 \\
25 \\
25 \\
25 \\
25 \\
25\end{array}$ & $\begin{array}{l}1725 \\
1725 \\
1726 \\
1726 \\
1727 \\
1727 \\
1728 \\
1728\end{array}$ & $\begin{array}{l}0.3 \\
3.1 \\
5.1 \\
10 \\
15 \\
20 \\
25 \\
29\end{array}$ & $\begin{array}{l}1,900 \\
1,900 \\
1,900 \\
1,900 \\
1,9000 \\
1,900 \\
1,900 \\
1,900\end{array}$ & $\begin{array}{l}476 \\
476 \\
476 \\
474 \\
469 \\
469 \\
469 \\
474\end{array}$ & $\begin{array}{l}7.9 \\
7.9 \\
7.9 \\
7.8 \\
7.7 \\
7.7 \\
7.6 \\
7.6\end{array}$ & $\begin{array}{l}29.3 \\
29.3 \\
29.2 \\
29.2 \\
28.9 \\
28.8 \\
28.8 \\
28.8\end{array}$ & $\begin{array}{l}- \\
- \\
-- \\
-- \\
- \\
- \\
-\end{array}$ & $\begin{array}{l}7.5 \\
7.5 \\
7.4 \\
7.4 \\
6.6 \\
6.3 \\
6.2 \\
6.2\end{array}$ & $\begin{array}{r}100 \\
100 \\
99 \\
98 \\
87 \\
83 \\
82 \\
81\end{array}$ \\
\hline
\end{tabular}


Table 22. Water-quality data for station 400913080421201, Ohio River at river mile 84.0, June to October, 1995, Continued.

[ft $=$ feet; $\mu \mathrm{s} / \mathrm{cm}=$ microsiemens per centimeter; ${ }^{\circ} \mathrm{C}=$ degrees Celsius;

$\mathrm{mg} / \mathrm{L}=\mathrm{mili}$ igrams per liter; - = data not collected]

\begin{tabular}{|c|c|c|c|c|c|c|c|c|c|}
\hline Date & Time & $\begin{array}{l}\text { Sampling } \\
\text { depth } \\
\text { (ft) }\end{array}$ & $\begin{array}{c}\text { Sample } \\
\text { location } \\
\text { (ft from } \\
\text { left bank) }\end{array}$ & $\begin{array}{l}\text { Specific } \\
\text { conduct- } \\
\text { ance } \\
(\mu \mathrm{s} / \mathrm{cm})\end{array}$ & $\begin{array}{l}\text { pH } \\
\text { (stan- } \\
\text { dard } \\
\text { units) }\end{array}$ & $\begin{array}{c}\text { Temper- } \\
\text { ature, } \\
\text { water } \\
\left({ }^{\circ} \mathrm{C}\right)\end{array}$ & $\begin{array}{l}\text { Trans- } \\
\text { parency } \\
\text { (Secchi } \\
\text { disk) } \\
(\mathrm{Et})\end{array}$ & $\begin{array}{l}\text { Dissolved } \\
\text { oxygen } \\
(\mathrm{mg} / \mathrm{L})\end{array}$ & $\begin{array}{c}\text { Dissolved } \\
\text { oxygen } \\
\text { (percent } \\
\text { satura- } \\
\text { tion) }\end{array}$ \\
\hline
\end{tabular}

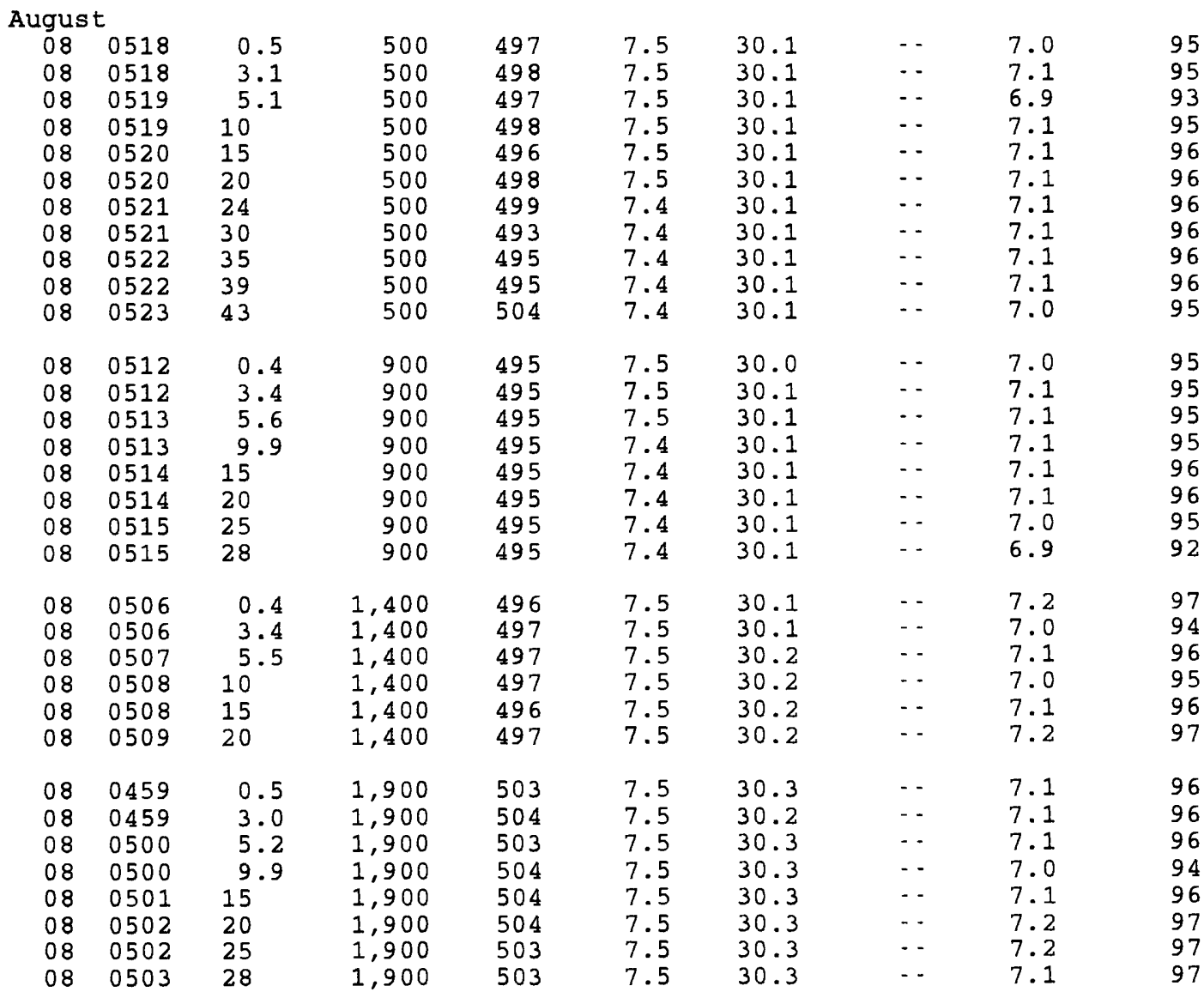


Table 22. Water-quality data for station 400913080421201, Ohio River at river mile 84.0, June to October, 1995, Continued.

[ft $=$ feet $; \mu \mathrm{s} / \mathrm{cm}=$ microsiemens per centimeter $;{ }^{\circ} \mathrm{C}=$ degrees Celsius; $\mathrm{mg} / \mathrm{L}=$ milligrams per liter; $\cdot$ - = data not collected]

\begin{tabular}{|c|c|c|c|c|c|c|c|c|c|}
\hline Date & Time & $\begin{array}{l}\text { Sampling } \\
\text { depth } \\
\text { (ft) }\end{array}$ & $\begin{array}{c}\text { Sample } \\
\text { location } \\
\text { (ft from } \\
\text { left bank) }\end{array}$ & $\begin{array}{l}\text { Specific } \\
\text { conduct- } \\
\text { ance } \\
(\mu \mathrm{S} / \mathrm{cm})\end{array}$ & $\begin{array}{l}\text { pH } \\
\text { (stan- } \\
\text { dard } \\
\text { units) }\end{array}$ & $\begin{array}{l}\text { Temper- } \\
\text { ature, } \\
\text { water } \\
\left({ }^{\circ} \mathrm{C}\right)\end{array}$ & $\begin{array}{l}\text { Trans- } \\
\text { parency } \\
\text { (Secchi } \\
\text { disk) } \\
(\mathrm{ft})\end{array}$ & $\begin{array}{l}\text { Dissolved } \\
\text { oxygen } \\
(\mathrm{mg} / \mathrm{L})\end{array}$ & $\begin{array}{c}\text { Dissolved } \\
\text { oxygen } \\
\text { (percent } \\
\text { satura- } \\
\text { tion) }\end{array}$ \\
\hline
\end{tabular}

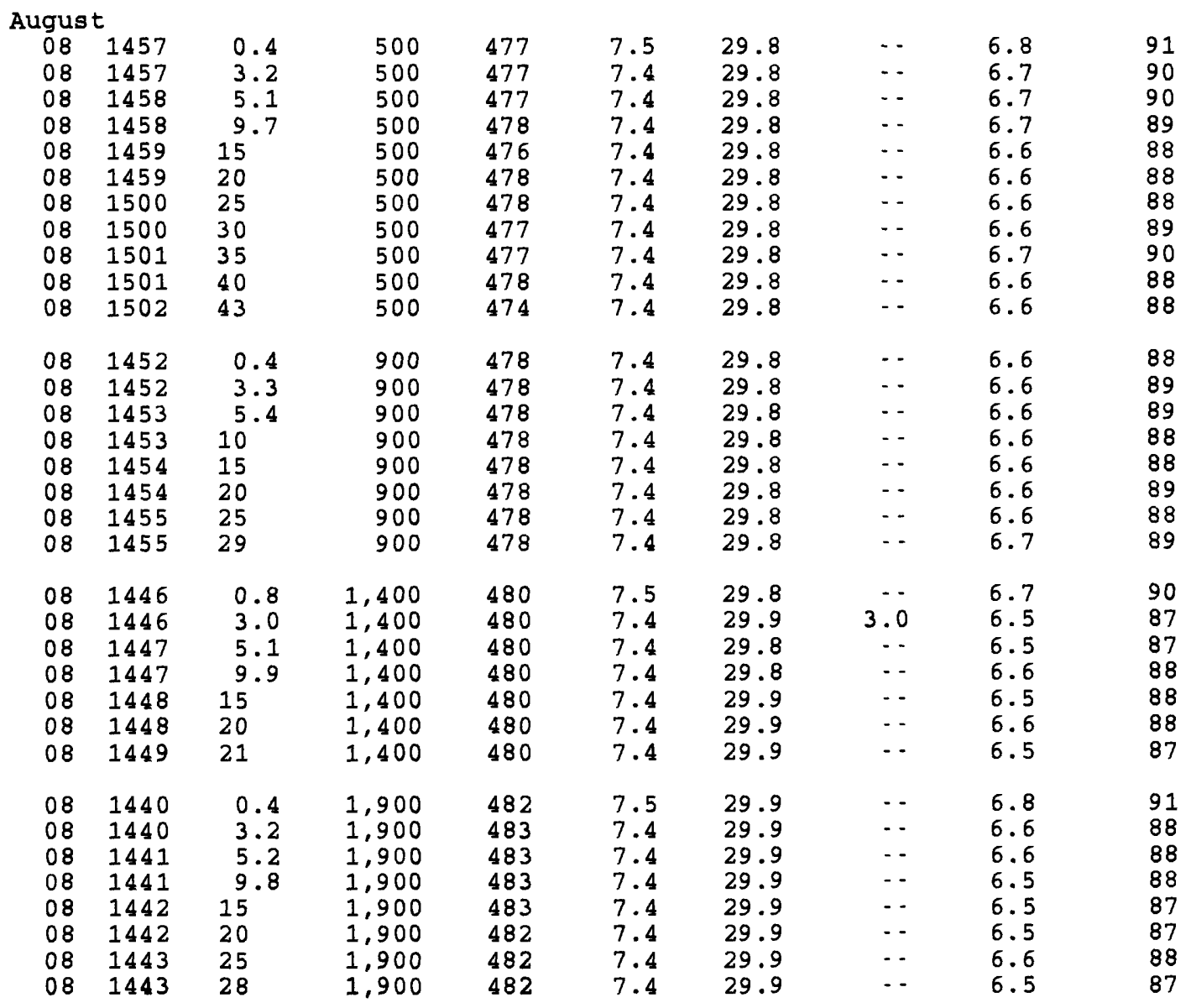


Table 22. Water-quality data for station 400913080421201 , Ohio River at river mile 84.0, June to October, 1995, Continued.

[ft = feet; $\mu \mathrm{s} / \mathrm{cm}=$ microsiemens per centimeter; ${ }^{\circ} \mathrm{C}=$ degrees Celsius; $\mathrm{mg} / \mathrm{L}=$ mililigrams per liter; - = data not collected]

\begin{tabular}{|c|c|c|c|c|c|c|c|c|c|}
\hline Date & Time & $\begin{array}{l}\text { Sampling } \\
\text { depth } \\
(f t)\end{array}$ & $\begin{array}{c}\text { Sample } \\
\text { location } \\
\text { (ft from } \\
\text { left bank) }\end{array}$ & $\begin{array}{l}\text { Specific } \\
\text { conduct - } \\
\text { ance } \\
(\mu \mathrm{s} / \mathrm{cm})\end{array}$ & $\begin{array}{l}\mathrm{pH} \\
\text { (stan- } \\
\text { dard } \\
\text { units) }\end{array}$ & $\begin{array}{c}\text { Temper- } \\
\text { ature, } \\
\text { water } \\
\left({ }^{\circ} \mathrm{C}\right)\end{array}$ & $\begin{array}{c}\text { Trans- } \\
\text { parency } \\
\text { (secchi } \\
\text { disk) } \\
\text { (ft) }\end{array}$ & $\begin{array}{l}\text { Dissolved } \\
\text { oxygen } \\
(\mathrm{mg} / \mathrm{L})\end{array}$ & $\begin{array}{c}\text { Dissolved } \\
\text { oxygen } \\
\text { (percent } \\
\text { satura- } \\
\text { tion) }\end{array}$ \\
\hline
\end{tabular}

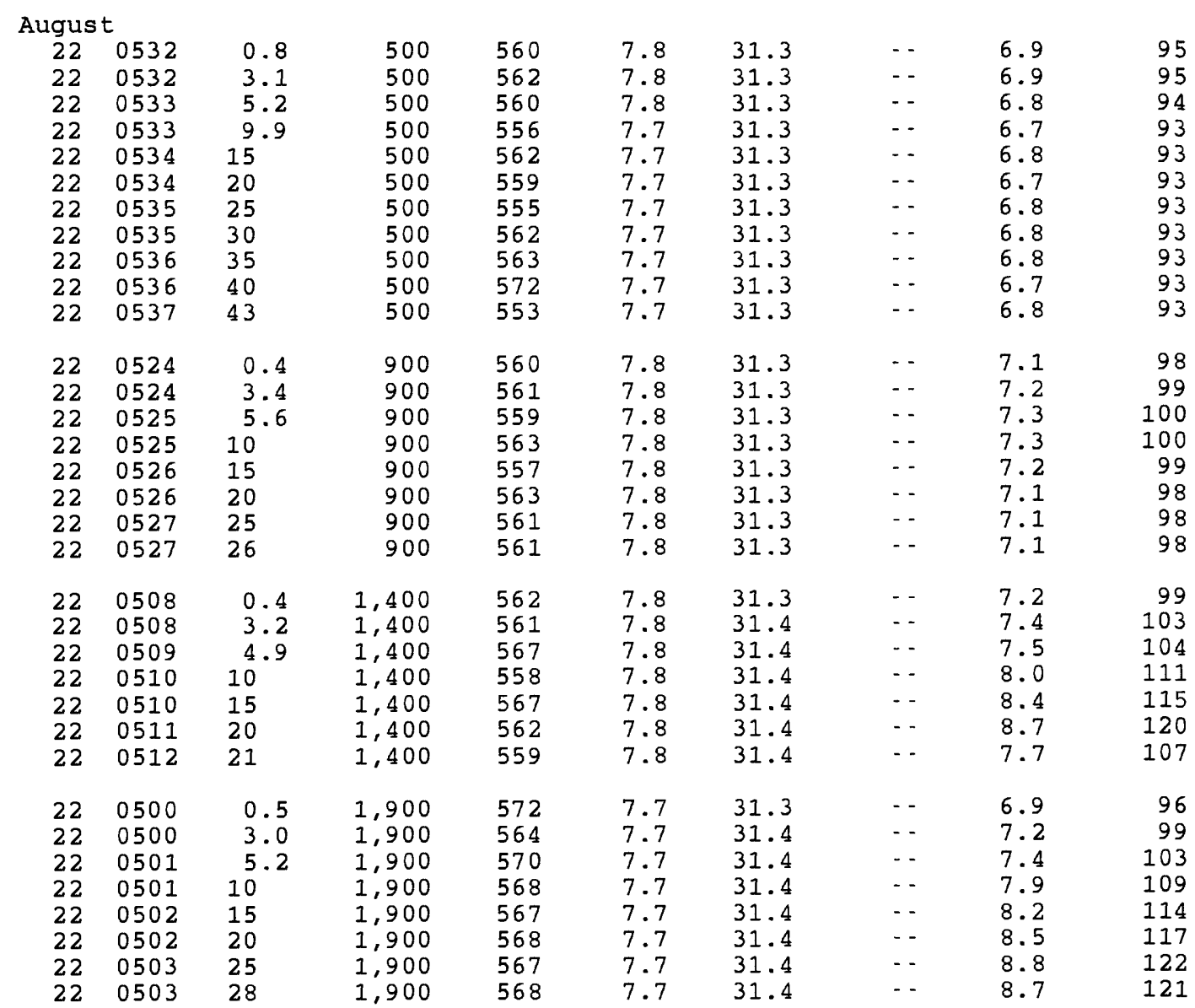


Table 22. Water-quality data for station 400913080421201 , Ohio River at river mile 84.0, June to October, 1995, Continued.

Ift $=$ feet; $\mu \mathrm{s} / \mathrm{cm}=$ microsiemens per centimeter; ${ }^{\circ} \mathrm{C}=$ degrees Celsius; $\mathrm{mg} / \mathrm{I}=\mathrm{mili}$ igrams per liter; - = data not collected]

\begin{tabular}{|c|c|c|c|c|c|c|c|c|c|}
\hline Date & Time & $\begin{array}{l}\text { Sarmpling } \\
\text { depth } \\
\text { (ft) }\end{array}$ & $\begin{array}{c}\text { Sample } \\
\text { location } \\
\text { (ft from } \\
\text { left bank) }\end{array}$ & $\begin{array}{l}\text { Specific } \\
\text { conduct- } \\
\text { ance } \\
(\mu s / \mathrm{cm})\end{array}$ & $\begin{array}{l}\mathrm{pH} \\
\text { (stan- } \\
\text { dard } \\
\text { units) }\end{array}$ & $\begin{array}{c}\text { Temper- } \\
\text { ature, } \\
\text { water } \\
\left({ }^{\circ} \mathrm{C}\right)\end{array}$ & $\begin{array}{l}\text { Trans- } \\
\text { parency } \\
\text { (secchi } \\
\text { disk) } \\
\text { (ft) }\end{array}$ & $\begin{array}{l}\text { Dissolved } \\
\text { oxygen } \\
(\mathrm{mg} / \mathrm{L})\end{array}$ & $\begin{array}{c}\text { Dissolved } \\
\text { oxygen } \\
\text { (percent } \\
\text { satura- } \\
\text { tion) }\end{array}$ \\
\hline
\end{tabular}

\begin{tabular}{|c|c|c|c|c|c|c|c|c|c|}
\hline $\begin{array}{c}\text { Augus } \\
22 \\
22 \\
22 \\
22 \\
22 \\
22 \\
22 \\
22 \\
22 \\
22 \\
22\end{array}$ & $\begin{array}{l}1631 \\
1631 \\
1632 \\
1632 \\
1633 \\
1633 \\
1634 \\
1634 \\
1635 \\
1635 \\
1636\end{array}$ & $\begin{array}{c}0.5 \\
3.4 \\
5.5 \\
9.8 \\
15 \\
20 \\
25 \\
30 \\
35 \\
40 \\
44\end{array}$ & $\begin{array}{l}500 \\
500 \\
500 \\
500 \\
500 \\
500 \\
500 \\
500 \\
500 \\
500 \\
500\end{array}$ & $\begin{array}{l}557 \\
557 \\
559 \\
558 \\
561 \\
557 \\
555 \\
558 \\
563 \\
554 \\
560\end{array}$ & $\begin{array}{l}7.9 \\
8.0 \\
8.0 \\
7.9 \\
7.9 \\
7.9 \\
7.9 \\
7.8 \\
7.8 \\
7.8 \\
7.8\end{array}$ & $\begin{array}{l}31.3 \\
31 \cdot 3 \\
31.3 \\
31 \cdot 3 \\
31.3 \\
31 \cdot 3 \\
31 \cdot 3 \\
31.3 \\
31 \cdot 3 \\
31.2 \\
31 \cdot 2\end{array}$ & $\begin{array}{l}- \\
- \\
\cdots \\
\cdots \\
\cdots \\
- \\
- \\
-\end{array}$ & $\begin{array}{l}7.6 \\
7.4 \\
7.6 \\
7.4 \\
7.3 \\
7.2 \\
7.3 \\
7.1 \\
7.0 \\
7.0 \\
6.9\end{array}$ & $\begin{array}{r}104 \\
102 \\
105 \\
101 \\
100 \\
99 \\
100 \\
97 \\
96 \\
96 \\
95\end{array}$ \\
\hline $\begin{array}{l}22 \\
22 \\
22 \\
22 \\
22 \\
22 \\
22 \\
22\end{array}$ & $\begin{array}{l}1625 \\
1625 \\
1626 \\
1626 \\
1627 \\
1627 \\
1628 \\
1628\end{array}$ & $\begin{array}{l}0.5 \\
3.5 \\
5.5 \\
9.9 \\
15 \\
20 \\
25 \\
29\end{array}$ & $\begin{array}{l}900 \\
900 \\
900 \\
900 \\
900 \\
900 \\
900 \\
900\end{array}$ & $\begin{array}{l}556 \\
557 \\
558 \\
560 \\
562 \\
555 \\
555 \\
564\end{array}$ & $\begin{array}{l}8.0 \\
8.0 \\
8.0 \\
7.9 \\
7.9 \\
7.9 \\
7.8 \\
7.7\end{array}$ & $\begin{array}{l}31.3 \\
31.3 \\
31.3 \\
31.3 \\
31.3 \\
31.3 \\
31.3 \\
31.2\end{array}$ & $\begin{array}{l}- \\
- \\
- \\
-- \\
-- \\
-\end{array}$ & $\begin{array}{l}7.8 \\
7.7 \\
7.6 \\
7.6 \\
7.3 \\
7.5 \\
7.3 \\
6.8\end{array}$ & $\begin{array}{r}107 \\
106 \\
105 \\
104 \\
100 \\
103 \\
100 \\
94\end{array}$ \\
\hline $\begin{array}{l}22 \\
22 \\
22 \\
22 \\
22 \\
22 \\
22\end{array}$ & $\begin{array}{l}1620 \\
1620 \\
1621 \\
1621 \\
1622 \\
1622 \\
1623\end{array}$ & $\begin{array}{l}0.4 \\
3.2 \\
5.4 \\
10 \\
15 \\
20 \\
22\end{array}$ & $\begin{array}{l}1,400 \\
1,400 \\
1,400 \\
1,400 \\
1,400 \\
1,400 \\
1,400\end{array}$ & $\begin{array}{l}555 \\
557 \\
558 \\
556 \\
557 \\
561 \\
556\end{array}$ & $\begin{array}{l}8.1 \\
8.1 \\
8.1 \\
8.0 \\
8.0 \\
7.9 \\
7.8\end{array}$ & $\begin{array}{l}31.4 \\
31.4 \\
31.4 \\
31.4 \\
31.3 \\
31.3 \\
31.3\end{array}$ & $\begin{array}{l}- \\
- \\
- \\
- \\
- \\
\cdots \\
-\end{array}$ & $\begin{array}{l}8.0 \\
8.0 \\
7.9 \\
7.7 \\
7.5 \\
7.2 \\
7.1\end{array}$ & $\begin{array}{r}110 \\
110 \\
108 \\
105 \\
103 \\
98 \\
97\end{array}$ \\
\hline $\begin{array}{l}22 \\
22 \\
22 \\
22 \\
22 \\
22 \\
22 \\
22\end{array}$ & $\begin{array}{l}1613 \\
1613 \\
1614 \\
1615 \\
1615 \\
1616 \\
1616 \\
1617\end{array}$ & $\begin{array}{l}0.7 \\
3.1 \\
5.1 \\
10 \\
15 \\
20 \\
25 \\
28\end{array}$ & $\begin{array}{l}1,900 \\
1,900 \\
1,900 \\
1,900 \\
1,900 \\
1,900 \\
1,900 \\
1,900\end{array}$ & $\begin{array}{l}556 \\
554 \\
554 \\
554 \\
554 \\
555 \\
563 \\
564\end{array}$ & $\begin{array}{l}8.2 \\
8.2 \\
8.2 \\
8.1 \\
8.1 \\
7.9 \\
7.7 \\
7.6\end{array}$ & $\begin{array}{l}31.5 \\
31.5 \\
31.5 \\
31.5 \\
31.4 \\
31.4 \\
31.4 \\
31.2\end{array}$ & $\begin{array}{l}-. \\
- \\
- \\
-- \\
-. \\
-.\end{array}$ & $\begin{array}{l}8.5 \\
8.3 \\
8.4 \\
7.8 \\
7.9 \\
7.5 \\
7.0 \\
6.5\end{array}$ & $\begin{array}{r}116 \\
114 \\
115 \\
108 \\
109 \\
103 \\
96 \\
89\end{array}$ \\
\hline
\end{tabular}


Table 22. Water-quality data for station 400913080421201 , Ohio River at river mile 84.0, June to October, 1995, Continued.

[Et $=$ feet; $\mu \mathrm{s} / \mathrm{cm}=$ microsiemens per centimeter; ${ }^{\circ} \mathrm{C}=$ degrees Celsius;

$\mathrm{mg} / \mathrm{L}=\mathrm{mili}$ igrams per liter; $\cdots$ = data not collected]

\begin{tabular}{|c|c|c|c|c|c|c|c|c|c|}
\hline Date & Time & $\begin{array}{l}\text { Sampling } \\
\text { depth } \\
\text { (ft) }\end{array}$ & $\begin{array}{c}\text { Sample } \\
\text { location } \\
\text { (ft from } \\
\text { left bank) }\end{array}$ & $\begin{array}{l}\text { Specific } \\
\text { conduct- } \\
\text { ance } \\
(\mu \mathrm{S} / \mathrm{cm})\end{array}$ & $\begin{array}{l}\text { pH } \\
\text { (stan- } \\
\text { dard } \\
\text { units) }\end{array}$ & $\begin{array}{l}\text { Temper- } \\
\text { ature, } \\
\text { water } \\
\left({ }^{\circ} \mathrm{C}\right)\end{array}$ & $\begin{array}{l}\text { Trans- } \\
\text { parency } \\
\text { (Secchi } \\
\text { disk) } \\
\text { (ft) }\end{array}$ & $\begin{array}{l}\text { Dissolved } \\
\text { oxygen } \\
(\mathrm{mg} / \mathrm{L})\end{array}$ & $\begin{array}{c}\text { Dissolved } \\
\text { oxygen } \\
\text { (percent } \\
\text { satura- } \\
\text { tion) }\end{array}$ \\
\hline
\end{tabular}

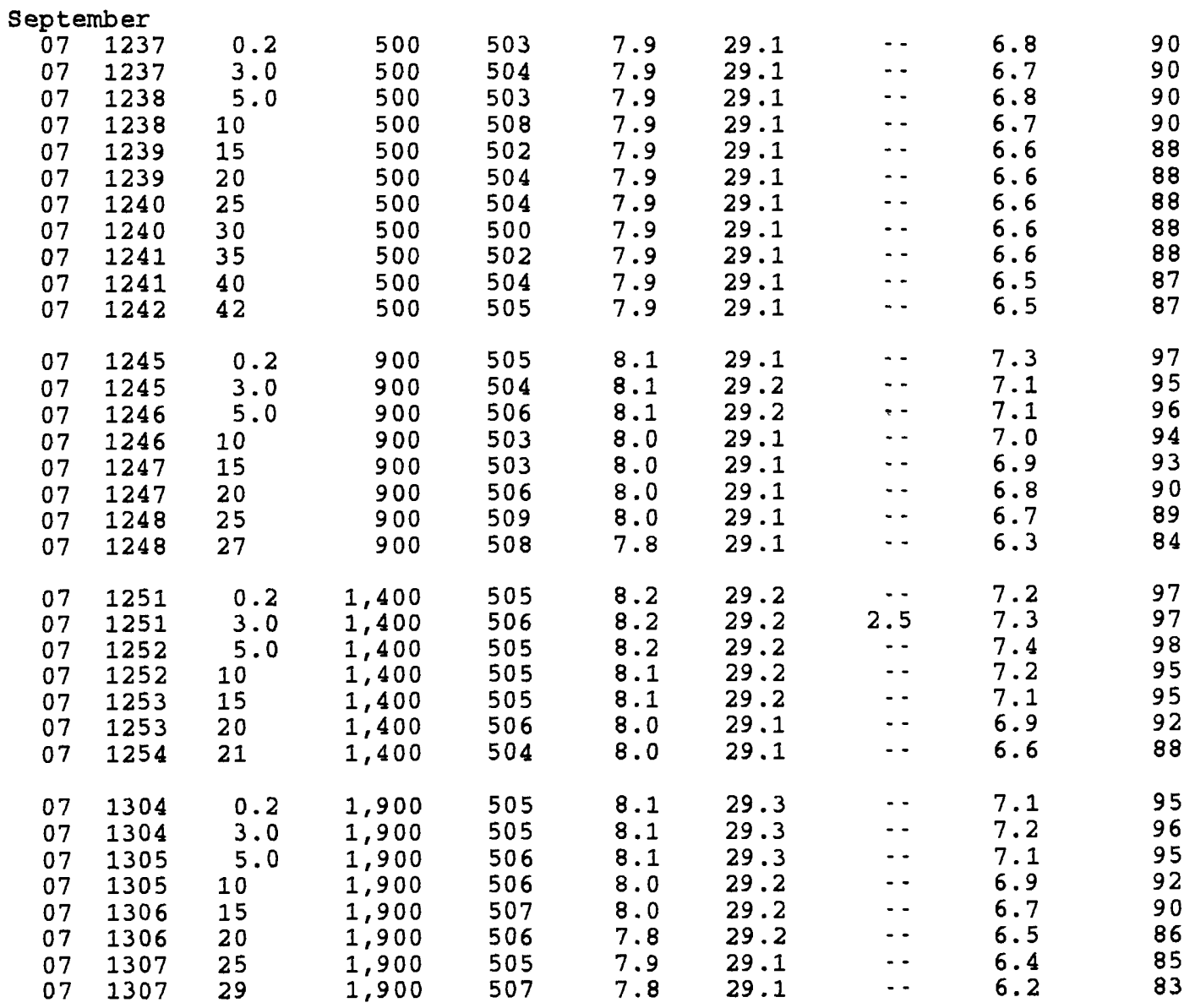


Table 22. Water-quality data for station 400913080421201, Ohio River at river mile 84.0, June to October, 1995, Continued.

[ft $=$ feet $; \mu \mathrm{S} / \mathrm{cm}=$ microsiemens per centimeter; ${ }^{\circ} \mathrm{C}=$ degrees Celsius; $\mathrm{mg} / \mathrm{L}=$ milligrams per liter; - - = data not collected]

\begin{tabular}{|c|c|c|c|c|c|c|c|c|c|}
\hline Date & Time & $\begin{array}{l}\text { Sampling } \\
\text { depth } \\
\text { (ft) }\end{array}$ & $\begin{array}{c}\text { Sample } \\
\text { location } \\
\text { (ft from } \\
\text { left bank) }\end{array}$ & $\begin{array}{l}\text { Specific } \\
\text { conduct- } \\
\text { ance } \\
(\mu \mathrm{s} / \mathrm{cm})\end{array}$ & $\begin{array}{l}\text { pH } \\
\text { (stan- } \\
\text { dard } \\
\text { units) }\end{array}$ & $\begin{array}{c}\text { Temper- } \\
\text { ature, } \\
\text { water } \\
\left({ }^{\circ} \mathrm{C}\right)\end{array}$ & $\begin{array}{c}\text { Trans- } \\
\text { parency } \\
\text { (Secchi } \\
\text { disk) } \\
\text { (ft) }\end{array}$ & $\begin{array}{l}\text { Dissolved } \\
\text { oxygen } \\
\text { (mg/L) }\end{array}$ & $\begin{array}{c}\text { Dissolved } \\
\text { oxygen } \\
\text { (percent } \\
\text { satura- } \\
\text { tion) }\end{array}$ \\
\hline
\end{tabular}

\begin{tabular}{|c|c|c|c|c|c|c|c|c|c|}
\hline $\begin{array}{c}\text { Septe } \\
08 \\
08 \\
08 \\
08 \\
08 \\
08 \\
08 \\
08 \\
08 \\
08\end{array}$ & $\begin{array}{l}\text { nber } \\
0439 \\
0439 \\
0440 \\
0440 \\
0441 \\
0441 \\
0442 \\
0442 \\
0443 \\
0444\end{array}$ & $\begin{array}{c}0.3 \\
2.8 \\
4.8 \\
9.9 \\
15 \\
19 \\
25 \\
30 \\
35 \\
38\end{array}$ & $\begin{array}{l}500 \\
500 \\
500 \\
500 \\
500 \\
500 \\
500 \\
500 \\
500 \\
500\end{array}$ & $\begin{array}{l}508 \\
508 \\
506 \\
509 \\
506 \\
506 \\
505 \\
516 \\
500 \\
518\end{array}$ & $\begin{array}{l}7.8 \\
7.8 \\
7.8 \\
7.5 \\
7.8 \\
7.8 \\
7.7 \\
7.9 \\
7.8 \\
7.8\end{array}$ & $\begin{array}{l}28.8 \\
28.9 \\
28.9 \\
29.0 \\
28.9 \\
28.9 \\
29.0 \\
29.0 \\
28.9 \\
29.0\end{array}$ & $\begin{array}{l}- \\
- \\
- \\
- \\
- \\
- \\
- \\
-\end{array}$ & $\begin{array}{l}6.7 \\
6.8 \\
6.7 \\
6.7 \\
6.7 \\
6.7 \\
6.7 \\
6.6 \\
6.6 \\
6.6\end{array}$ & $\begin{array}{l}89 \\
90 \\
89 \\
89 \\
89 \\
89 \\
88 \\
88 \\
88 \\
88\end{array}$ \\
\hline $\begin{array}{l}08 \\
08 \\
08 \\
08 \\
08 \\
08 \\
08\end{array}$ & $\begin{array}{l}0454 \\
0455 \\
0455 \\
0456 \\
0456 \\
0457 \\
0457\end{array}$ & $\begin{array}{l}0.4 \\
2.7 \\
4.8 \\
10 \\
15 \\
20 \\
21\end{array}$ & 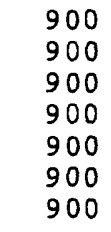 & $\begin{array}{l}505 \\
503 \\
507 \\
505 \\
510 \\
509 \\
505\end{array}$ & $\begin{array}{l}7.9 \\
7.9 \\
7.9 \\
7.9 \\
7.9 \\
7.9 \\
7.9\end{array}$ & $\begin{array}{l}29.0 \\
29.0 \\
29.0 \\
29.0 \\
29.0 \\
29.0 \\
29.0\end{array}$ & $\begin{array}{l}-- \\
-- \\
- \\
-- \\
- \\
-\end{array}$ & $\begin{array}{l}6.8 \\
6.8 \\
6.8 \\
6.8 \\
6.8 \\
6.8 \\
6.7\end{array}$ & $\begin{array}{l}90 \\
90 \\
91 \\
90 \\
90 \\
91 \\
89\end{array}$ \\
\hline $\begin{array}{l}08 \\
08 \\
08 \\
08 \\
08 \\
08 \\
08 \\
08\end{array}$ & $\begin{array}{l}0448 \\
0448 \\
0449 \\
0449 \\
0450 \\
0450 \\
0451 \\
0451\end{array}$ & $\begin{array}{l}0.3 \\
3.0 \\
4.9 \\
9.9 \\
15 \\
20 \\
25 \\
27\end{array}$ & $\begin{array}{l}1,400 \\
1,400 \\
1,400 \\
1,400 \\
1,400 \\
1,400 \\
1,400 \\
1,400\end{array}$ & $\begin{array}{l}504 \\
506 \\
508 \\
506 \\
513 \\
503 \\
507 \\
514\end{array}$ & $\begin{array}{l}7.8 \\
7.9 \\
7.9 \\
7.9 \\
7.9 \\
7.9 \\
7.9 \\
7.9\end{array}$ & $\begin{array}{l}29.0 \\
29.0 \\
29.0 \\
29.0 \\
29.0 \\
29.0 \\
29.0 \\
29.0\end{array}$ & $\begin{array}{l}-- \\
-- \\
-- \\
-- \\
-- \\
-- \\
-\end{array}$ & $\begin{array}{l}6.7 \\
6.8 \\
6.8 \\
6.8 \\
6.8 \\
6.8 \\
6.9 \\
6.7\end{array}$ & $\begin{array}{l}90 \\
90 \\
90 \\
90 \\
91 \\
91 \\
91 \\
90\end{array}$ \\
\hline $\begin{array}{l}08 \\
08 \\
08 \\
08 \\
08 \\
08 \\
08\end{array}$ & $\begin{array}{l}0500 \\
0500 \\
0501 \\
0501 \\
0502 \\
0502 \\
0503\end{array}$ & $\begin{array}{l}0.6 \\
3.1 \\
5.0 \\
10 \\
15 \\
20 \\
25\end{array}$ & $\begin{array}{l}1,900 \\
1,900 \\
1,900 \\
1,900 \\
1,900 \\
1,900 \\
1,900\end{array}$ & $\begin{array}{l}508 \\
508 \\
512 \\
510 \\
513 \\
514 \\
506\end{array}$ & $\begin{array}{l}7.8 \\
7.8 \\
7.8 \\
7.8 \\
7.8 \\
7.8 \\
7.8\end{array}$ & $\begin{array}{l}29.0 \\
29.0 \\
29.0 \\
29.0 \\
29.0 \\
29.0 \\
29.0\end{array}$ & $\begin{array}{l}- \\
- \\
-- \\
-- \\
-- \\
-\end{array}$ & $\begin{array}{l}6.6 \\
6.7 \\
6.8 \\
6.7 \\
6.8 \\
6.5 \\
6.5\end{array}$ & $\begin{array}{l}88 \\
89 \\
90 \\
89 \\
90 \\
87 \\
87\end{array}$ \\
\hline $\begin{array}{l}22 \\
22 \\
22 \\
22 \\
22 \\
22 \\
22 \\
22 \\
22 \\
22 \\
22\end{array}$ & $\begin{array}{l}1901 \\
1901 \\
1902 \\
1902 \\
1903 \\
1903 \\
1904 \\
1904 \\
1905 \\
1905 \\
1906\end{array}$ & $\begin{array}{r}0.7 \\
3.1 \\
5.0 \\
9.8 \\
15 \\
20 \\
25 \\
30 \\
35 \\
40 \\
45\end{array}$ & $\begin{array}{l}500 \\
500 \\
500 \\
500 \\
500 \\
500 \\
500 \\
500 \\
500 \\
500 \\
500\end{array}$ & $\begin{array}{l}525 \\
525 \\
526 \\
526 \\
526 \\
526 \\
526 \\
526 \\
526 \\
527 \\
527\end{array}$ & $\begin{array}{l}7.5 \\
7.5 \\
7.5 \\
7.5 \\
7.5 \\
7.5 \\
7.5 \\
7.6 \\
7.5 \\
7.5 \\
7.5\end{array}$ & $\begin{array}{l}24.5 \\
24.6 \\
24.6 \\
24.6 \\
24.6 \\
24.6 \\
24.6 \\
24.6 \\
24.6 \\
24.6 \\
24.6\end{array}$ & $\begin{array}{l}-- \\
-- \\
-- \\
-- \\
-- \\
-- \\
-- \\
-- \\
-\end{array}$ & $\begin{array}{l}6.9 \\
6.9 \\
6.9 \\
6.9 \\
6.9 \\
6.9 \\
6.9 \\
6.8 \\
6.8 \\
6.8 \\
6.8\end{array}$ & $\begin{array}{l}84 \\
84 \\
84 \\
84 \\
84 \\
84 \\
84 \\
84 \\
83 \\
83 \\
83\end{array}$ \\
\hline
\end{tabular}


Table 22. Water-quality data for station 400913080421201, Ohio River at river mile 84.0, June to October, 1995, Continued.

lft $=$ feet $; \mu \mathrm{s} / \mathrm{cm}=$ microsiemens per centimeter; ${ }^{\circ} \mathrm{C}=$ degrees Celsius; $\mathrm{mg} / \mathrm{L}=$ mililigrams per 1 iter; $\cdots$ = data not collected]

\begin{tabular}{|c|c|c|c|c|c|c|c|c|c|}
\hline Date & Time & $\begin{array}{l}\text { Sampling } \\
\text { depth } \\
\text { (ft) }\end{array}$ & $\begin{array}{l}\text { sample } \\
\text { location } \\
\text { (ft from } \\
\text { left bank) }\end{array}$ & $\begin{array}{l}\text { specific } \\
\text { conduct- } \\
\text { ance } \\
(\mu s / \mathrm{cm})\end{array}$ & $\begin{array}{l}\text { pH } \\
\text { (stan- } \\
\text { dard } \\
\text { units) }\end{array}$ & $\begin{array}{c}\text { Temper- } \\
\text { ature, } \\
\text { water } \\
\left({ }^{\circ} \mathrm{C}\right)\end{array}$ & $\begin{array}{c}\text { Trans - } \\
\text { parency } \\
\text { (secchi } \\
\text { disk) } \\
\text { (ft) }\end{array}$ & $\begin{array}{l}\text { Dissolved } \\
\text { oxygen } \\
(\mathrm{mg} / \mathrm{L})\end{array}$ & $\begin{array}{c}\text { Dissolved } \\
\text { oxygen } \\
\text { (percent } \\
\text { satura- } \\
\text { tion) }\end{array}$ \\
\hline
\end{tabular}

\begin{tabular}{|c|c|c|c|c|c|c|c|c|c|}
\hline \multicolumn{2}{|c|}{ September } & & & & & & & & \\
\hline $\begin{array}{l}22 \\
22 \\
22 \\
22 \\
22 \\
22 \\
22\end{array}$ & $\begin{array}{l}1853 \\
1853 \\
1854 \\
1854 \\
1855 \\
1855 \\
1856\end{array}$ & $\begin{array}{l}0.9 \\
3.1 \\
5.0 \\
9.8 \\
15 \\
20 \\
25\end{array}$ & $\begin{array}{l}900 \\
900 \\
900 \\
900 \\
900 \\
900 \\
900\end{array}$ & $\begin{array}{l}528 \\
528 \\
528 \\
528 \\
527 \\
527 \\
527\end{array}$ & $\begin{array}{l}7.6 \\
7.6 \\
7.6 \\
7.6 \\
7.6 \\
7.5 \\
7.5\end{array}$ & $\begin{array}{l}24.6 \\
24.6 \\
24.6 \\
24.6 \\
24.6 \\
24.6 \\
24.6\end{array}$ & $\begin{array}{l}\cdots \\
\cdots \\
\cdots \\
\cdots \\
\cdots\end{array}$ & $\begin{array}{l}6.9 \\
6.8 \\
6.9 \\
6.8 \\
6.8 \\
6.8 \\
6.8\end{array}$ & $\begin{array}{l}84 \\
84 \\
84 \\
84 \\
84 \\
83 \\
83\end{array}$ \\
\hline $\begin{array}{l}22 \\
22 \\
22 \\
22 \\
22 \\
22 \\
22\end{array}$ & $\begin{array}{l}1844 \\
1844 \\
1845 \\
1845 \\
1846 \\
1846 \\
1847\end{array}$ & $\begin{array}{l}1.1 \\
3.1 \\
4.9 \\
9.7 \\
15 \\
20 \\
21\end{array}$ & $\begin{array}{l}1,400 \\
1,400 \\
1,400 \\
1,400 \\
1,400 \\
1,400 \\
1,400\end{array}$ & $\begin{array}{l}528 \\
528 \\
528 \\
528 \\
528 \\
529 \\
529\end{array}$ & $\begin{array}{l}7.6 \\
7.5 \\
7.5 \\
7.5 \\
7.5 \\
7.5 \\
7.5\end{array}$ & $\begin{array}{l}24.6 \\
24.7 \\
24.7 \\
24.7 \\
24.7 \\
24.7 \\
24.6\end{array}$ & $\begin{array}{l}\cdots \\
\cdots \\
\cdots \\
\cdots \\
-\end{array}$ & $\begin{array}{l}6.8 \\
6.8 \\
6.8 \\
6.8 \\
6.8 \\
6.7 \\
6.7\end{array}$ & $\begin{array}{l}83 \\
83 \\
83 \\
83 \\
83 \\
82 \\
82\end{array}$ \\
\hline $\begin{array}{l}22 \\
22 \\
22 \\
22 \\
22 \\
22 \\
22 \\
22\end{array}$ & $\begin{array}{l}1836 \\
1836 \\
1837 \\
1837 \\
1838 \\
1838 \\
1839 \\
1839\end{array}$ & $\begin{array}{l}1.0 \\
3.0 \\
5.0 \\
9.9 \\
15 \\
20 \\
25 \\
29\end{array}$ & $\begin{array}{l}1,900 \\
1,900 \\
1,900 \\
1,900 \\
1,900 \\
1,900 \\
1,900 \\
1,900\end{array}$ & $\begin{array}{l}527 \\
527 \\
527 \\
527 \\
527 \\
527 \\
527 \\
528\end{array}$ & $\begin{array}{l}7.5 \\
7.5 \\
7.5 \\
7.5 \\
7.5 \\
7.5 \\
7.5 \\
7.5\end{array}$ & $\begin{array}{l}24.7 \\
24.7 \\
24.7 \\
24.7 \\
24.7 \\
24.7 \\
24.7 \\
24.7\end{array}$ & $\begin{array}{l}\cdots \\
\cdots \\
\cdots \\
\cdots \\
\cdots \\
\cdots\end{array}$ & $\begin{array}{l}6.7 \\
6.7 \\
6.7 \\
6.6 \\
6.7 \\
6.7 \\
6.7 \\
6.7\end{array}$ & $\begin{array}{l}82 \\
82 \\
82 \\
81 \\
82 \\
82 \\
82 \\
81\end{array}$ \\
\hline $\begin{array}{c}\text { Octob } \\
03 \\
03 \\
03 \\
03 \\
03 \\
03 \\
03 \\
03 \\
03 \\
03 \\
03\end{array}$ & $\begin{array}{l}e r \\
1001 \\
1001 \\
1002 \\
1002 \\
1003 \\
1004 \\
1004 \\
1005 \\
1005 \\
1006 \\
1007\end{array}$ & $\begin{array}{c}0.7 \\
2.9 \\
4.8 \\
9.8 \\
15 \\
20 \\
25 \\
30 \\
35 \\
40 \\
44\end{array}$ & $\begin{array}{l}500 \\
500 \\
500 \\
5000 \\
5000 \\
500 \\
5000 \\
5000 \\
5000 \\
5000 \\
500\end{array}$ & $\begin{array}{l}518 \\
518 \\
518 \\
519 \\
518 \\
518 \\
518 \\
518 \\
519 \\
518 \\
520\end{array}$ & $\begin{array}{l}7.5 \\
7.5 \\
7.5 \\
7.5 \\
7.5 \\
7.5 \\
7.5 \\
7.6 \\
7.6 \\
7.5 \\
7.4\end{array}$ & $\begin{array}{l}23.2 \\
23.2 \\
23.2 \\
23.2 \\
23.2 \\
23.2 \\
23.2 \\
23.2 \\
23.3 \\
23.2 \\
23.2\end{array}$ & $\begin{array}{l}\cdots \\
\ldots \\
\cdots \\
\cdots \\
\cdots \\
\cdots \\
\cdots\end{array}$ & $\begin{array}{l}6.7 \\
6.7 \\
5.6 \\
6.2 \\
5.6 \\
6.8 \\
6.8 \\
6.8 \\
6.8 \\
6.8 \\
6.8\end{array}$ & $\begin{array}{l}81 \\
80 \\
68 \\
74 \\
67 \\
81 \\
82 \\
82 \\
81 \\
81 \\
82\end{array}$ \\
\hline
\end{tabular}


Table 22. Water-quality data for station 400913080421201 , Ohio River at river mile 84.0, June to October, 1995, Continued.

$\left[\mathrm{ft}=\mathrm{feet} ; \mu \mathrm{S} / \mathrm{cm}=\mathrm{microsiemens}\right.$ per centimeter $;{ }^{\circ} \mathrm{C}=$ degrees celsius;
$\mathrm{mg} / \mathrm{L}=\mathrm{milligrams}$ per liter; $\cdots=$ data not collected]

\begin{tabular}{|c|c|c|c|c|c|c|c|c|c|}
\hline Date & Time & $\begin{array}{l}\text { Sampling } \\
\text { depth } \\
\text { (ft) }\end{array}$ & $\begin{array}{c}\text { Sample } \\
\text { location } \\
\text { (ft from } \\
\text { left bank) }\end{array}$ & $\begin{array}{l}\text { Specific } \\
\text { conduct- } \\
\text { ance } \\
(\mu \mathrm{s} / \mathrm{cm})\end{array}$ & $\begin{array}{l}\mathrm{pH} \\
\text { (stan- } \\
\text { dard } \\
\text { units) }\end{array}$ & $\begin{array}{c}\text { Temper- } \\
\text { ature, } \\
\text { water } \\
\left({ }^{\circ} \mathrm{C}\right)\end{array}$ & $\begin{array}{c}\text { Trans - } \\
\text { parency } \\
\text { (secchi } \\
\text { disk) } \\
\text { (ft) }\end{array}$ & $\begin{array}{l}\text { Dissolved } \\
\text { oxygen } \\
\text { (mg/L) }\end{array}$ & $\begin{array}{c}\text { Dissolved } \\
\text { oxygen } \\
\text { (percent } \\
\text { satura- } \\
\text { tion) }\end{array}$ \\
\hline
\end{tabular}

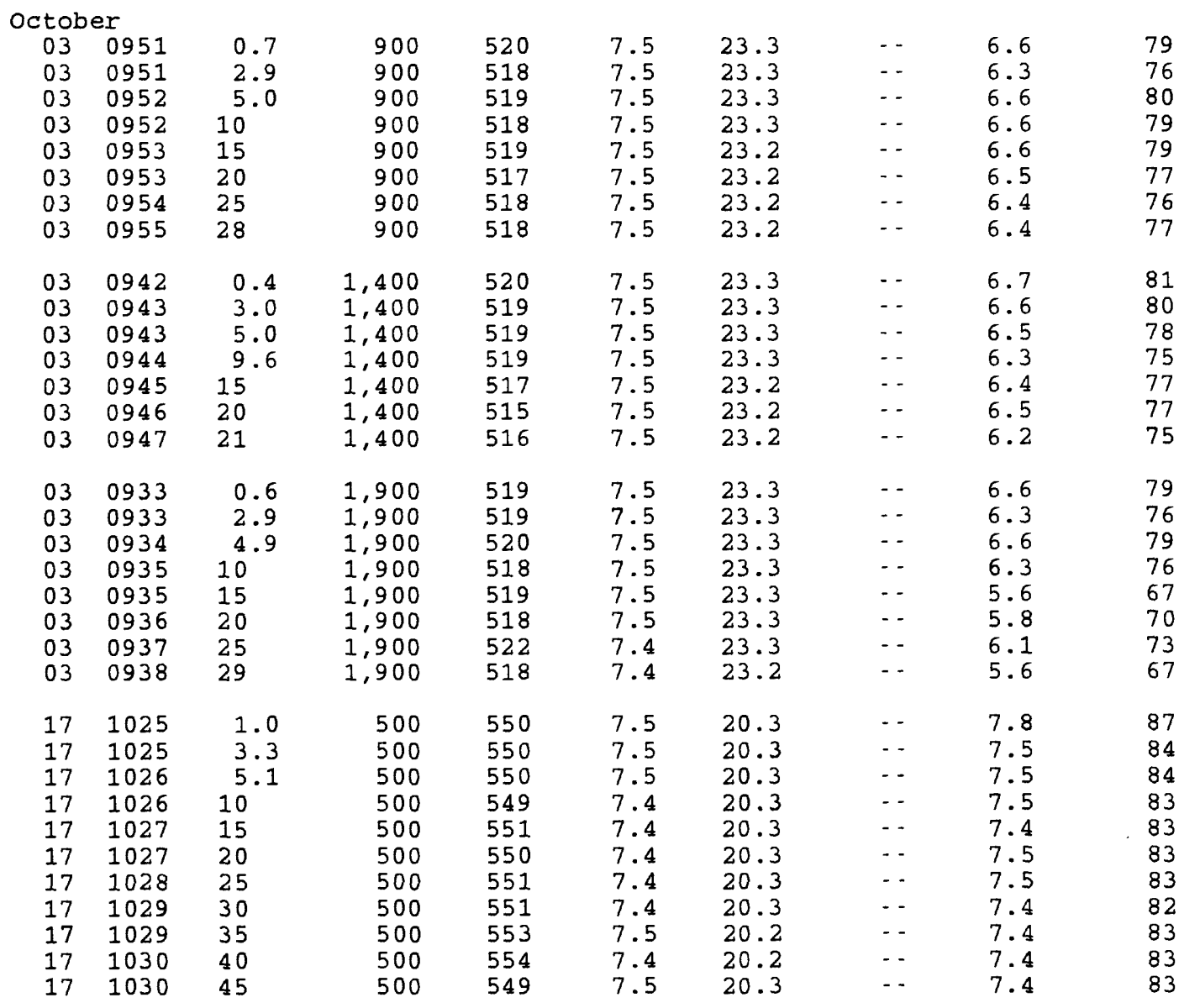


Table 22. Water-quality data for station 400913080421201, Ohio River at river mile 84.0, June to October, 1995, Continued.

[ft $=$ feet $; \mu \mathrm{s} / \mathrm{cm}=$ microsiemens per centimeter $;{ }^{\circ} \mathrm{C}=$ degrees Celsius; $\mathrm{mg} / \mathrm{L}=$ milligrams per liter; - = data not collected]

\begin{tabular}{|c|c|c|c|c|c|c|c|c|c|}
\hline Date & Time & $\begin{array}{l}\text { Sampling } \\
\text { depth } \\
\text { (ft) }\end{array}$ & $\begin{array}{c}\text { Sample } \\
\text { location } \\
\text { (ft from } \\
\text { left bank) }\end{array}$ & $\begin{array}{l}\text { Specific } \\
\text { conduct- } \\
\text { ance } \\
(\mu \mathrm{s} / \mathrm{cm})\end{array}$ & $\begin{array}{l}\text { pH } \\
\text { (stan- } \\
\text { dard } \\
\text { units) }\end{array}$ & $\begin{array}{c}\text { Temper- } \\
\text { ature, } \\
\text { water } \\
\left({ }^{\circ} \mathrm{C}\right)\end{array}$ & $\begin{array}{l}\text { Trans- } \\
\text { parency } \\
\text { (Secchi } \\
\text { disk) } \\
\text { (ft) }\end{array}$ & $\begin{array}{l}\text { Dissolved } \\
\text { oxygen } \\
(\mathrm{mg} / \mathrm{L})\end{array}$ & $\begin{array}{c}\text { Dissolved } \\
\text { oxygen } \\
\text { (percent } \\
\text { satura- } \\
\text { tion) }\end{array}$ \\
\hline \multicolumn{10}{|c|}{ October } \\
\hline 17 & 1020 & 0.6 & 900 & 551 & 7.4 & 20.3 & $\cdots$ & 7.7 & 86 \\
\hline 17 & 1020 & 3.4 & 900 & 550 & 7.4 & 20.3 & - - & 7.7 & 86 \\
\hline 17 & 1021 & 5.2 & 900 & 551 & 7.4 & 20.3 & 3.5 & 7.7 & 86 \\
\hline 17 & 1021 & 10 & 900 & 551 & 7.4 & 20.3 & - - & 7.7 & 86 \\
\hline 17 & 1022 & 15 & 900 & 551 & 7.4 & 20.3 & $\cdots$ & 7.7 & 86 \\
\hline 17 & 1022 & 20 & 900 & 550 & 7.4 & 20.3 & -- & 7.6 & 85 \\
\hline 17 & 1023 & 25 & 900 & 551 & 7.4 & 20.3 & - & 7.6 & 85 \\
\hline 17 & 1023 & 27 & 900 & 550 & 7.4 & 20.3 & - & 7.7 & 85 \\
\hline 17 & 1015 & 0.6 & 1,400 & 552 & 7.4 & 20.3 & - & 7.6 & 85 \\
\hline 17 & 1015 & 3.3 & 1,400 & 552 & 7.4 & 20.3 & -- & 7.7 & 86 \\
\hline 17 & 1016 & 5.1 & 1,400 & 552 & 7.4 & 20.3 & $\cdots$ & 7.7 & 86 \\
\hline 17 & 1016 & 10 & 1,400 & 552 & 7.4 & 20.4 & -- & 7.7 & 86 \\
\hline 17 & 1017 & 15 & 1,400 & 552 & 7.4 & 20.4 & -- & 7.7 & 86 \\
\hline 17 & 1017 & 20 & 1,400 & 552 & 7.4 & 20.3 & -- & 7.7 & 86 \\
\hline 17 & 1018 & 22 & 1,400 & 553 & 7.4 & 20.3 & - & 7.5 & 84 \\
\hline 17 & 1009 & 0.8 & 1,900 & 548 & 7.3 & 20.3 & $\cdots$ & 7.9 & 88 \\
\hline 17 & 1009 & 3.0 & 1,900 & 549 & 7.4 & 20.3 & -- & 7.7 & 86 \\
\hline 17 & 1010 & 5.2 & 1,900 & 550 & 7.4 & 20.4 & $\cdots$ & 7.7 & 86 \\
\hline 17 & 1010 & 10 & 1,900 & 550 & 7.4 & 20.3 & -- & 7.6 & 85 \\
\hline 17 & 1011 & 15 & 1,900 & 549 & 7.4 & 20.3 & -- & 7.6 & 84 \\
\hline 17 & 1011 & 20 & 1,900 & 549 & 7.4 & 20.3 & - & 7.7 & 86 \\
\hline 17 & 1012 & 25 & 1,900 & 550 & 7.4 & 20.3 & -- & 7.6 & 85 \\
\hline 17 & 1012 & 29 & 1,900 & 550 & 7.3 & 20.4 & - & 7.3 & 82 \\
\hline
\end{tabular}


Table 23. Daily maximum, minimum, and mean specific conductance at station 403155080373501, from the New Cumberland Dam (upstream) continuous-recording water-quality monitor, June to October 1995

$[\cdots$, value not determined]

\begin{tabular}{|c|c|c|c|c|c|c|c|c|c|}
\hline \multirow{3}{*}{ Day } & \multicolumn{9}{|c|}{ Specific conductance, in microsiemens per centimeter at 25 degrees celsius } \\
\hline & \multicolumn{3}{|c|}{ May } & \multicolumn{3}{|c|}{ June } & \multicolumn{3}{|c|}{ July } \\
\hline & Maximum & Minimum & Mean & Maximum & Minimum & Mean & Maximum & Minimum & Mean \\
\hline $\begin{array}{l}1 \\
2 \\
3 \\
4 \\
5\end{array}$ & $\begin{array}{l}\cdots \\
\cdots \\
\cdots \\
\cdots\end{array}$ & $\begin{array}{l}\cdots \\
\cdots \\
\cdots \\
\cdots \\
\cdots\end{array}$ & $\begin{array}{l}\cdots \\
\cdots \\
\cdots \\
\cdots\end{array}$ & $\begin{array}{c}\cdots \\
\cdots \\
\cdots \\
\cdots \\
\end{array}$ & $\begin{array}{c}\cdots \\
\cdots \\
\cdots \\
\cdots \\
\cdots\end{array}$ & $\begin{array}{c}\cdots \\
\cdots \\
\cdots \\
\cdots \\
\cdots\end{array}$ & $\begin{array}{l}404 \\
421 \\
432 \\
425 \\
411\end{array}$ & $\begin{array}{l}386 \\
392 \\
421 \\
395 \\
396\end{array}$ & $\begin{array}{l}394 \\
404 \\
428 \\
409 \\
403\end{array}$ \\
\hline $\begin{array}{r}6 \\
7 \\
8 \\
9 \\
10\end{array}$ & $\begin{array}{l}\cdots \\
\cdots \\
\cdots \\
\cdots\end{array}$ & $\begin{array}{l}\cdots \\
\cdots \\
\cdots \\
\cdots \\
\cdots\end{array}$ & $\begin{array}{l}\cdots \\
\cdots \\
\cdots \\
\cdots\end{array}$ & $\begin{array}{c}\cdots \\
\cdots \\
\cdots \\
\cdots \\
\cdots\end{array}$ & $\begin{array}{c}\cdots \\
\cdots \\
\cdots \\
\cdots \\
\cdots\end{array}$ & $\begin{array}{c}\cdots \\
\cdots \\
\cdots \\
\cdots \\
\cdots\end{array}$ & $\begin{array}{l}412 \\
413 \\
416 \\
420 \\
433\end{array}$ & $\begin{array}{l}407 \\
408 \\
409 \\
414 \\
420\end{array}$ & $\begin{array}{l}409 \\
410 \\
411 \\
417 \\
427\end{array}$ \\
\hline $\begin{array}{l}11 \\
12 \\
13 \\
14 \\
15\end{array}$ & $\begin{array}{l}\cdots \\
\cdots \\
\cdots \\
\cdots\end{array}$ & $\begin{array}{l}\cdots \\
\cdots \\
\cdots \\
\cdots \\
\cdots\end{array}$ & $\begin{array}{l}\cdots \\
\cdots \\
\cdots \\
\cdots\end{array}$ & $\begin{array}{c}\cdots \\
\cdots \\
\cdots \\
\cdots \\
\cdots\end{array}$ & $\begin{array}{c}\cdots \\
\cdots \\
\cdots \\
\cdots \\
\cdots\end{array}$ & $\begin{array}{c}\cdots \\
\cdots \\
\cdots \\
\cdots\end{array}$ & $\begin{array}{l}438 \\
437 \\
445 \\
445 \\
441\end{array}$ & $\begin{array}{l}422 \\
427 \\
436 \\
440 \\
430\end{array}$ & $\begin{array}{l}429 \\
433 \\
441 \\
443 \\
435\end{array}$ \\
\hline $\begin{array}{l}16 \\
17 \\
18 \\
19 \\
20\end{array}$ & $\begin{array}{l}\cdots \\
\cdots \\
\cdots \\
\cdots\end{array}$ & $\begin{array}{l}\cdots \\
\cdots \\
\cdots \\
\cdots\end{array}$ & $\begin{array}{c}\cdots \\
\cdots \\
\cdots \\
\cdots \\
\cdots\end{array}$ & $\begin{array}{l}\ldots \\
308 \\
302 \\
295 \\
295\end{array}$ & $\begin{array}{l}\ldots \\
299 \\
294 \\
292 \\
293\end{array}$ & $\begin{array}{l}302 \\
298 \\
293 \\
294\end{array}$ & $\begin{array}{l}442 \\
476 \\
473 \\
463 \\
461\end{array}$ & $\begin{array}{l}428 \\
442 \\
459 \\
456 \\
414\end{array}$ & $\begin{array}{l}432 \\
463 \\
465 \\
459 \\
434\end{array}$ \\
\hline $\begin{array}{l}21 \\
22 \\
23 \\
24 \\
25\end{array}$ & $\begin{array}{l}\cdots \\
\cdots \\
\cdots \\
\cdots\end{array}$ & $\begin{array}{l}\cdots \\
\cdots \\
\cdots \\
\cdots \\
\cdots\end{array}$ & $\begin{array}{c}\cdots \\
\cdots \\
\cdots \\
\cdots \\
\cdots\end{array}$ & $\begin{array}{l}298 \\
321 \\
333 \\
351 \\
364\end{array}$ & $\begin{array}{l}294 \\
297 \\
320 \\
333 \\
351\end{array}$ & $\begin{array}{l}295 \\
310 \\
324 \\
344 \\
357\end{array}$ & $\begin{array}{l}414 \\
413 \\
417 \\
427 \\
435\end{array}$ & $\begin{array}{l}411 \\
408 \\
411 \\
417 \\
424\end{array}$ & $\begin{array}{l}413 \\
410 \\
414 \\
421 \\
429\end{array}$ \\
\hline $\begin{array}{l}26 \\
27 \\
28 \\
29 \\
30 \\
31\end{array}$ & $\begin{array}{l}\cdots \\
\cdots \\
\cdots \\
\cdots \\
\cdots\end{array}$ & $\begin{array}{l}\cdots \\
\cdots \\
\cdots \\
\cdots \\
\cdots\end{array}$ & $\begin{array}{c}\cdots \\
\cdots \\
\cdots \\
\cdots \\
\cdots\end{array}$ & $\begin{array}{l}371 \\
395 \\
408 \\
404 \\
401\end{array}$ & $\begin{array}{l}364 \\
369 \\
387 \\
370 \\
372\end{array}$ & $\begin{array}{l}368 \\
381 \\
396 \\
389 \\
383 \\
\ldots .-\end{array}$ & $\begin{array}{l}445 \\
449 \\
455 \\
455 \\
454 \\
456\end{array}$ & $\begin{array}{l}435 \\
445 \\
448 \\
452 \\
451 \\
454\end{array}$ & $\begin{array}{l}441 \\
447 \\
452 \\
454 \\
453 \\
455\end{array}$ \\
\hline Month & $\ldots$ & $\ldots$ & $\ldots$ & $\ldots$ & $\ldots$ & $\ldots$ & 476 & 386 & 430 \\
\hline
\end{tabular}


Table 23. Daily maximum, minimum, and mean specific conductance at station 403155080373501, from the New Cumberland Dam (upstream) continuous-recording water-quality monitor, June to october 1995, Continued.

$$
[\cdots, \text { value not determined] }
$$

\begin{tabular}{|c|c|c|c|c|c|c|c|c|c|}
\hline \multirow{3}{*}{ Day } & \multicolumn{3}{|c|}{ Specific conductance, } & nicrosiem & nens per & \multirow{2}{*}{ centimeter } & \multicolumn{3}{|c|}{25 degrees Celsius } \\
\hline & \multicolumn{3}{|c|}{ August } & \multicolumn{2}{|c|}{ September } & & \multicolumn{3}{|c|}{ October } \\
\hline & Maximum & Minimum & Mean & Maximum & Minimum & Mean & Maximum & Minimum & Mean \\
\hline $\begin{array}{l}1 \\
2 \\
3 \\
4 \\
5\end{array}$ & $\begin{array}{l}460 \\
466 \\
477 \\
484 \\
484\end{array}$ & $\begin{array}{l}455 \\
459 \\
457 \\
477 \\
481\end{array}$ & $\begin{array}{l}457 \\
462 \\
471 \\
481 \\
483\end{array}$ & $\begin{array}{l}467 \\
466 \\
465 \\
466 \\
468\end{array}$ & $\begin{array}{l}461 \\
464 \\
463 \\
461 \\
463\end{array}$ & $\begin{array}{l}464 \\
465 \\
464 \\
464 \\
465\end{array}$ & $\begin{array}{l}486 \\
484 \\
486 \\
486 \\
503\end{array}$ & $\begin{array}{l}481 \\
481 \\
481 \\
483 \\
485\end{array}$ & $\begin{array}{l}483 \\
482 \\
483 \\
485 \\
491\end{array}$ \\
\hline $\begin{array}{r}6 \\
7 \\
8 \\
9 \\
10\end{array}$ & $\begin{array}{l}484 \\
476 \\
461 \\
462 \\
466\end{array}$ & $\begin{array}{l}476 \\
461 \\
448 \\
450 \\
462\end{array}$ & $\begin{array}{l}481 \\
469 \\
453 \\
456 \\
464\end{array}$ & $\begin{array}{l}477 \\
483 \\
484 \\
488 \\
493\end{array}$ & $\begin{array}{l}467 \\
477 \\
478 \\
483 \\
487\end{array}$ & $\begin{array}{l}472 \\
480 \\
481 \\
485 \\
489\end{array}$ & $\begin{array}{l}520 \\
524 \\
518 \\
517 \\
523\end{array}$ & $\begin{array}{l}503 \\
518 \\
513 \\
513 \\
516\end{array}$ & $\begin{array}{l}514 \\
521 \\
516 \\
515 \\
520\end{array}$ \\
\hline $\begin{array}{l}11 \\
12 \\
13 \\
14 \\
15\end{array}$ & $\begin{array}{l}490 \\
542 \\
551 \\
551 \\
536\end{array}$ & $\begin{array}{l}466 \\
490 \\
542 \\
536 \\
524\end{array}$ & $\begin{array}{l}471 \\
530 \\
547 \\
545 \\
530\end{array}$ & $\begin{array}{l}496 \\
497 \\
496 \\
491 \\
494\end{array}$ & $\begin{array}{l}492 \\
495 \\
491 \\
489 \\
489\end{array}$ & $\begin{array}{l}495 \\
496 \\
494 \\
490 \\
491\end{array}$ & $\begin{array}{l}525 \\
525 \\
518 \\
515 \\
521\end{array}$ & $\begin{array}{l}521 \\
518 \\
514 \\
512 \\
511\end{array}$ & $\begin{array}{l}523 \\
522 \\
516 \\
513 \\
515\end{array}$ \\
\hline $\begin{array}{l}16 \\
17 \\
18 \\
19 \\
20\end{array}$ & $\begin{array}{l}524 \\
516 \\
504 \\
490 \\
478\end{array}$ & $\begin{array}{l}516 \\
504 \\
488 \\
477 \\
476\end{array}$ & $\begin{array}{l}520 \\
510 \\
496 \\
483 \\
477\end{array}$ & $\begin{array}{l}495 \\
496 \\
497 \\
494 \\
492\end{array}$ & $\begin{array}{l}491 \\
491 \\
493 \\
491 \\
489\end{array}$ & $\begin{array}{l}493 \\
494 \\
495 \\
492 \\
490\end{array}$ & $\begin{array}{l}522 \\
517 \\
477 \\
467 \\
482\end{array}$ & $\begin{array}{l}517 \\
477 \\
462 \\
461 \\
467\end{array}$ & $\begin{array}{l}521 \\
502 \\
467 \\
464 \\
474\end{array}$ \\
\hline $\begin{array}{l}21 \\
22 \\
23 \\
24 \\
25\end{array}$ & $\begin{array}{l}478 \\
484 \\
484 \\
478 \\
477\end{array}$ & $\begin{array}{l}475 \\
474 \\
477 \\
476 \\
476\end{array}$ & $\begin{array}{l}477 \\
480 \\
480 \\
477 \\
476\end{array}$ & $\begin{array}{l}490 \\
505 \\
504 \\
504 \\
500\end{array}$ & $\begin{array}{l}486 \\
485 \\
502 \\
500 \\
497\end{array}$ & $\begin{array}{l}488 \\
493 \\
503 \\
502 \\
498\end{array}$ & $\begin{array}{l}493 \\
493 \\
486 \\
496 \\
499\end{array}$ & $\begin{array}{l}480 \\
485 \\
479 \\
482 \\
495\end{array}$ & $\begin{array}{l}489 \\
491 \\
482 \\
490 \\
498\end{array}$ \\
\hline $\begin{array}{l}26 \\
27 \\
28 \\
29 \\
30 \\
31\end{array}$ & $\begin{array}{l}480 \\
480 \\
478 \\
476 \\
475 \\
471\end{array}$ & $\begin{array}{l}477 \\
478 \\
474 \\
473 \\
471 \\
460\end{array}$ & $\begin{array}{l}478 \\
479 \\
476 \\
475 \\
473 \\
466\end{array}$ & $\begin{array}{l}498 \\
497 \\
494 \\
494 \\
492 \\
\ldots\end{array}$ & $\begin{array}{l}496 \\
493 \\
493 \\
492 \\
486 \\
\ldots .\end{array}$ & $\begin{array}{l}497 \\
495 \\
493 \\
493 \\
489 \\
--\end{array}$ & $\begin{array}{l}498 \\
493 \\
492 \\
498 \\
501 \\
499\end{array}$ & $\begin{array}{l}493 \\
490 \\
488 \\
488 \\
496 \\
479\end{array}$ & $\begin{array}{l}495 \\
491 \\
490 \\
493 \\
499 \\
485\end{array}$ \\
\hline Month & 551 & 448 & 485 & 505 & 461 & 487 & 525 & 461 & 498 \\
\hline
\end{tabular}


Table 24. Daily maximum, minimum, and median $p H$ at station 403155080373501, from the New Cumberland Dam (upstream) continuous-recording water-quality monitor, June to October 1995

$[\cdots$, value not determined]

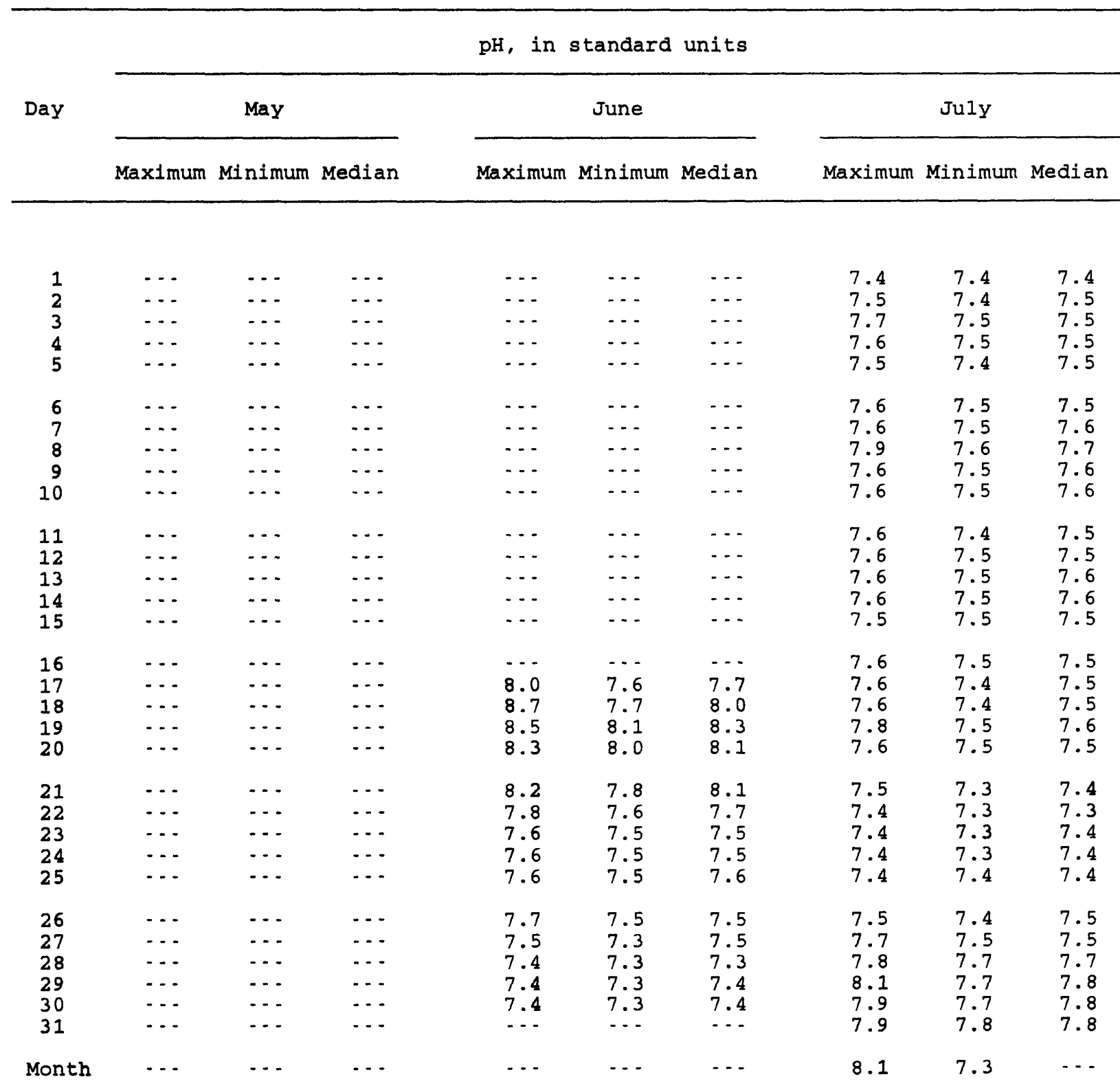


Table 24. Daily maximum, minimum, and median $p H$ at station 403155080373501, from the New Cumberland Dam (upstream) continuous-recording water-quality monitor, June to October 1995, Continued.

$[\cdots$, value not determined]

$\mathrm{pH}$, in standard units

Day $\frac{\text { August }}{\text { Maximum Minimum Median }} \quad \frac{\text { September }}{\text { Maximum Minimum Median }} \quad$ Maximum Minimum Median

\begin{tabular}{|c|c|c|c|c|c|c|c|c|c|}
\hline $\begin{array}{l}1 \\
2 \\
3 \\
4 \\
5\end{array}$ & $\begin{array}{l}8.0 \\
8.0 \\
7.9 \\
7.9 \\
7.6\end{array}$ & $\begin{array}{l}7.8 \\
7.8 \\
7.8 \\
7.6 \\
7.5\end{array}$ & $\begin{array}{l}7.9 \\
7.9 \\
7.9 \\
7.8 \\
7.5\end{array}$ & $\begin{array}{l}8.1 \\
8.0 \\
8.0 \\
8.1 \\
8.2\end{array}$ & $\begin{array}{l}7.8 \\
7.8 \\
7.7 \\
7.8 \\
7.9\end{array}$ & $\begin{array}{l}7.9 \\
7.9 \\
7.8 \\
7.9 \\
8.0\end{array}$ & $\begin{array}{l}7.4 \\
7.4 \\
7.6 \\
7.6 \\
7.6\end{array}$ & $\begin{array}{l}7.4 \\
7.4 \\
7.4 \\
7.5 \\
7.5\end{array}$ & $\begin{array}{l}7.4 \\
7.4 \\
7.4 \\
7.6 \\
7.6\end{array}$ \\
\hline $\begin{array}{r}6 \\
7 \\
8 \\
9 \\
10\end{array}$ & $\begin{array}{l}7.5 \\
7.6 \\
7.6 \\
7.5 \\
7.6\end{array}$ & $\begin{array}{l}7.4 \\
7.5 \\
7.4 \\
7.4 \\
7.4\end{array}$ & $\begin{array}{l}7.5 \\
7.5 \\
7.5 \\
7.5 \\
7.4\end{array}$ & $\begin{array}{l}8.2 \\
7.9 \\
8.0 \\
7.9 \\
8.1\end{array}$ & $\begin{array}{l}7.9 \\
7.8 \\
7.8 \\
7.8 \\
7.9\end{array}$ & $\begin{array}{l}8.0 \\
7.9 \\
7.9 \\
7.9 \\
7.9\end{array}$ & $\begin{array}{l}7.6 \\
7.6 \\
7.6 \\
7.6 \\
7.6\end{array}$ & $\begin{array}{l}7.6 \\
7.6 \\
7.6 \\
7.6 \\
7.6\end{array}$ & $\begin{array}{l}7.6 \\
7.6 \\
7.6 \\
7.6 \\
7.6\end{array}$ \\
\hline $\begin{array}{l}11 \\
12 \\
13 \\
14 \\
15\end{array}$ & $\begin{array}{l}7.7 \\
7.9 \\
7.7 \\
7.6 \\
7.7\end{array}$ & $\begin{array}{l}7.5 \\
7.5 \\
7.6 \\
7.6 \\
7.6\end{array}$ & $\begin{array}{l}7.5 \\
7.7 \\
7.6 \\
7.6 \\
7.6\end{array}$ & $\begin{array}{l}7.9 \\
7.7 \\
7.7 \\
8.1 \\
7.9\end{array}$ & $\begin{array}{l}7.7 \\
7.6 \\
7.6 \\
7.6 \\
7.6\end{array}$ & $\begin{array}{l}7.7 \\
7.7 \\
7.7 \\
7.7 \\
7.7\end{array}$ & $\begin{array}{l}7.6 \\
7.6 \\
7.7 \\
7.6 \\
7.7\end{array}$ & $\begin{array}{l}7.6 \\
7.6 \\
7.6 \\
7.6 \\
7.6\end{array}$ & $\begin{array}{l}7.6 \\
7.6 \\
7.6 \\
7.6 \\
7.6\end{array}$ \\
\hline $\begin{array}{l}16 \\
17 \\
18 \\
19 \\
20\end{array}$ & $\begin{array}{l}8.1 \\
8.0 \\
7.8 \\
7.6 \\
7.6\end{array}$ & $\begin{array}{l}7.6 \\
7.7 \\
7.6 \\
7.6 \\
7.6\end{array}$ & $\begin{array}{l}7.7 \\
7.8 \\
7.7 \\
7.6 \\
7.6\end{array}$ & $\begin{array}{l}7.6 \\
7.6 \\
7.6 \\
7.5 \\
7.5\end{array}$ & $\begin{array}{l}7.6 \\
7.6 \\
7.5 \\
7.5 \\
7.5\end{array}$ & $\begin{array}{l}7.6 \\
7.6 \\
7.6 \\
7.5 \\
7.5\end{array}$ & $\begin{array}{l}7.7 \\
7.7 \\
7.6 \\
7.6 \\
7.6\end{array}$ & $\begin{array}{l}7.6 \\
7.5 \\
7.5 \\
7.6 \\
7.5\end{array}$ & $\begin{array}{l}7.6 \\
7.5 \\
7.5 \\
7.6 \\
7.6\end{array}$ \\
\hline $\begin{array}{l}21 \\
22 \\
23 \\
24 \\
25\end{array}$ & $\begin{array}{l}7.7 \\
7.8 \\
7.8 \\
7.7 \\
7.9\end{array}$ & $\begin{array}{l}7.6 \\
7.6 \\
7.6 \\
7.6 \\
7.7\end{array}$ & $\begin{array}{l}7.6 \\
7.6 \\
7.7 \\
7.6 \\
7.7\end{array}$ & $\begin{array}{l}7.5 \\
7.5 \\
7.3 \\
7.3 \\
7.4\end{array}$ & $\begin{array}{l}7.5 \\
7.3 \\
7.2 \\
7.3 \\
7.3\end{array}$ & $\begin{array}{l}7.5 \\
7.5 \\
7.3 \\
7.3 \\
7.3\end{array}$ & $\begin{array}{l}7.6 \\
7.6 \\
7.7 \\
7.7 \\
7.7\end{array}$ & $\begin{array}{l}7.6 \\
7.6 \\
7.6 \\
7.6 \\
7.6\end{array}$ & $\begin{array}{l}7.6 \\
7.6 \\
7.7 \\
7.7 \\
7.7\end{array}$ \\
\hline $\begin{array}{l}26 \\
27 \\
28 \\
29 \\
30 \\
31\end{array}$ & $\begin{array}{l}7.8 \\
8.1 \\
8.1 \\
8.1 \\
8.0 \\
7.9\end{array}$ & $\begin{array}{l}7.7 \\
7.8 \\
7.9 \\
7.9 \\
7.9 \\
7.8\end{array}$ & $\begin{array}{l}7.8 \\
8.0 \\
8.0 \\
7.9 \\
7.9 \\
7.8\end{array}$ & $\begin{array}{l}7.4 \\
7.4 \\
7.4 \\
7.4 \\
7.4 \\
\cdots .\end{array}$ & $\begin{array}{l}7.3 \\
7.4 \\
7.4 \\
7.4 \\
7.4 \\
\cdots .\end{array}$ & $\begin{array}{l}7.3 \\
7.4 \\
7.4 \\
7.4 \\
7.4\end{array}$ & $\begin{array}{l}7.8 \\
7.8 \\
7.8 \\
7.7 \\
7.7 \\
7.7\end{array}$ & $\begin{array}{l}7.7 \\
7.7 \\
7.7 \\
7.7 \\
7.7 \\
7.7\end{array}$ & $\begin{array}{l}7.7 \\
7.8 \\
7.7 \\
7.7 \\
7.7 \\
7.7\end{array}$ \\
\hline Month & 8.1 & 7.4 & & 8.2 & 7.2 & $\cdots$ & 7.8 & 7.4 & $\cdots$ \\
\hline
\end{tabular}


Table 25. Daily maximum, minimum, and mean water temperature at station 403155080373501, from the New Cumberland Dam (upstream) continuous-recording water-quality monitor, June to October 1995

\begin{tabular}{cccc}
\hline Water temperature, in degrees Celsius \\
\cline { 2 - 4 } & May & June & Ju1y \\
\cline { 2 - 3 } & Maximum Minimum Mean & Maximum Minimum Mean & Maximum Minimum Mean \\
\hline
\end{tabular}

\begin{tabular}{|c|c|c|c|c|c|c|c|c|c|}
\hline $\begin{array}{l}1 \\
2 \\
3 \\
4 \\
5\end{array}$ & $\begin{array}{l}\cdots \\
\cdots \\
\cdots \\
\cdots\end{array}$ & $\begin{array}{l}\cdots \\
\cdots \\
\cdots \\
\cdots\end{array}$ & $\begin{array}{l}\cdots \\
\cdots \\
\cdots \\
\cdots\end{array}$ & $\begin{array}{l}\cdots \\
\cdots \\
\cdots \\
\cdots\end{array}$ & $\begin{array}{l}\cdots \\
\cdots \\
\cdots \\
\cdots\end{array}$ & $\begin{array}{l}\cdots \\
\cdots \\
\cdots \\
\cdots\end{array}$ & $\begin{array}{l}25.2 \\
25.2 \\
26.9 \\
25.6 \\
29.0\end{array}$ & $\begin{array}{l}24.7 \\
24.8 \\
24.9 \\
24.6 \\
25.2\end{array}$ & $\begin{array}{l}24.9 \\
25.0 \\
25.5 \\
25.0 \\
27.1\end{array}$ \\
\hline $\begin{array}{r}6 \\
7 \\
8 \\
9 \\
10\end{array}$ & $\begin{array}{l}\cdots \\
\cdots \\
\cdots\end{array}$ & $\begin{array}{c}\cdots \\
\cdots \\
\cdots \\
\cdots\end{array}$ & $\begin{array}{c}\cdots \\
\cdots \\
\cdots \\
\cdots\end{array}$ & $\begin{array}{l}\cdots \\
\cdots \\
\cdots \\
\cdots\end{array}$ & $\begin{array}{l}\cdots \\
\cdots \\
\cdots \\
\cdots\end{array}$ & $\begin{array}{l}\cdots \\
\cdots \\
\cdots \\
\cdots\end{array}$ & $\begin{array}{l}29.0 \\
28.7 \\
28.5 \\
27.2 \\
29.3\end{array}$ & $\begin{array}{l}27.6 \\
27.7 \\
26.4 \\
26.0 \\
27.0\end{array}$ & $\begin{array}{l}28.4 \\
28.3 \\
27.5 \\
26.5 \\
28.1\end{array}$ \\
\hline $\begin{array}{l}11 \\
12 \\
13 \\
14 \\
15\end{array}$ & $\begin{array}{l}\cdots \\
\cdots \\
\cdots \\
\cdots\end{array}$ & $\begin{array}{c}\cdots \\
\cdots \\
\cdots \\
\cdots\end{array}$ & $\begin{array}{l}\cdots \\
\cdots \\
\cdots \\
\cdots\end{array}$ & $\begin{array}{l}\cdots \\
\cdots \\
\cdots \\
\cdots\end{array}$ & $\begin{array}{l}\cdots \\
\cdots \\
\cdots \\
\cdots\end{array}$ & $\begin{array}{l}\cdots \\
\cdots \\
\cdots \\
\cdots \\
\cdots\end{array}$ & $\begin{array}{l}30.3 \\
30.8 \\
30.6 \\
31.3 \\
31.7\end{array}$ & $\begin{array}{l}27.8 \\
28.3 \\
29.2 \\
29.4 \\
30.7\end{array}$ & $\begin{array}{l}29.1 \\
29.6 \\
30.0 \\
30.5 \\
31.1\end{array}$ \\
\hline $\begin{array}{l}16 \\
17 \\
18 \\
19 \\
20\end{array}$ & $\begin{array}{c}\cdots \\
\cdots \\
\cdots\end{array}$ & $\begin{array}{c}\cdots \\
\cdots \\
\cdots \\
\cdots\end{array}$ & $\begin{array}{l}\cdots \\
\cdots \\
\cdots \\
\cdots\end{array}$ & $\begin{array}{r}\cdots \\
23.5 \\
26.7 \\
27.3 \\
28.0\end{array}$ & $\begin{array}{l}22.7 \\
23.0 \\
25.6 \\
26.4\end{array}$ & $\begin{array}{r}23.0 \\
24.6 \\
26.5 \\
27.3\end{array}$ & $\begin{array}{l}31.5 \\
30.1 \\
30.0 \\
30.6 \\
30.5\end{array}$ & $\begin{array}{l}29.8 \\
26.9 \\
27.5 \\
28.5 \\
29.4\end{array}$ & $\begin{array}{l}30.4 \\
28.3 \\
28.9 \\
29.5 \\
29.9\end{array}$ \\
\hline $\begin{array}{l}21 \\
22 \\
23 \\
24 \\
25\end{array}$ & $\begin{array}{l}\cdots \\
\cdots \\
\cdots \\
\cdots\end{array}$ & $\begin{array}{c}\cdots \\
\cdots \\
\cdots \\
\cdots\end{array}$ & $\begin{array}{l}\cdots \\
\cdots \\
\cdots \\
\cdots\end{array}$ & $\begin{array}{l}28.1 \\
28.0 \\
28.3 \\
29.4 \\
29.4\end{array}$ & $\begin{array}{l}27.0 \\
26.4 \\
25.1 \\
27.6 \\
27.3\end{array}$ & $\begin{array}{l}27.7 \\
27.4 \\
27.0 \\
28.5 \\
28.3\end{array}$ & $\begin{array}{l}30.8 \\
31.5 \\
31.5 \\
31.7 \\
32.1\end{array}$ & $\begin{array}{l}29.1 \\
30.0 \\
29.8 \\
30.7 \\
30.7\end{array}$ & $\begin{array}{l}30.1 \\
30.8 \\
30.7 \\
31.3 \\
31.5\end{array}$ \\
\hline $\begin{array}{l}26 \\
27 \\
28 \\
29 \\
30 \\
31\end{array}$ & $\begin{array}{l}\cdots \\
\cdots \\
\cdots \\
\cdots \\
\cdots\end{array}$ & $\begin{array}{l}\cdots \\
\cdots \\
\cdots \\
\cdots \\
\cdots\end{array}$ & $\begin{array}{c}\cdots \\
\cdots \\
\cdots \\
\cdots \\
\cdots\end{array}$ & $\begin{array}{l}29.0 \\
29.0 \\
26.0 \\
27.7 \\
24.8\end{array}$ & $\begin{array}{l}25.1 \\
25.1 \\
24.9 \\
24.6 \\
24.5\end{array}$ & $\begin{array}{r}27.1 \\
25.8 \\
25.3 \\
25.9 \\
24.6 \\
\ldots . .\end{array}$ & $\begin{array}{l}32.0 \\
31.7 \\
31.7 \\
32.9 \\
33.1 \\
33.5\end{array}$ & $\begin{array}{l}31.0 \\
30.8 \\
29.9 \\
30.7 \\
30.6 \\
31.3\end{array}$ & $\begin{array}{l}31.6 \\
31.3 \\
31.0 \\
31.9 \\
31.8 \\
32.5\end{array}$ \\
\hline Month & $\cdots$ & $\cdots$ & $\cdots$ & $\cdots$ & $\cdots$ & $\cdots$ & 33.5 & 24.6 & 29.3 \\
\hline
\end{tabular}


Table 25. Daily maximum, minimum, and mean water temperature at station 403155080373501, from the New Cumberland Dam (upstream) continuous-recording water-quality monitor, June to October 1995, Continued.

$[\cdots$, value not determined]

\begin{tabular}{|c|c|c|c|c|c|c|c|c|c|}
\hline \multirow{3}{*}{ Day } & \multicolumn{9}{|c|}{ Water temperature, in degrees Celsius } \\
\hline & \multicolumn{3}{|c|}{ August } & \multicolumn{3}{|c|}{ September } & \multicolumn{3}{|c|}{ October } \\
\hline & Maximum & Minimum & Mean & Maximum & Minimum & Mean & Maximum & Minimum & Mean \\
\hline $\begin{array}{l}1 \\
2 \\
3 \\
4 \\
5\end{array}$ & $\begin{array}{l}33.1 \\
33.4 \\
33.9 \\
33.9 \\
33.6\end{array}$ & $\begin{array}{l}31.9 \\
32.0 \\
32.7 \\
33.2 \\
31.7\end{array}$ & $\begin{array}{l}32.6 \\
32.9 \\
33.4 \\
33.5 \\
32.9\end{array}$ & $\begin{array}{l}33.8 \\
32.9 \\
32.3 \\
32.8 \\
32.4\end{array}$ & $\begin{array}{l}32.7 \\
31.6 \\
31.0 \\
30.8 \\
30.5\end{array}$ & $\begin{array}{l}33.3 \\
32.3 \\
31.7 \\
31.9 \\
31.6\end{array}$ & $\begin{array}{l}24.9 \\
25.8 \\
25.7 \\
25.5 \\
24.4\end{array}$ & $\begin{array}{l}23.8 \\
24.2 \\
24.5 \\
24.0 \\
21.5\end{array}$ & $\begin{array}{l}24.3 \\
25.0 \\
25.1 \\
24.7 \\
23.3\end{array}$ \\
\hline $\begin{array}{r}6 \\
7 \\
8 \\
9 \\
10\end{array}$ & $\begin{array}{l}32.2 \\
30.1 \\
29.8 \\
31.2 \\
31.5\end{array}$ & $\begin{array}{l}30.1 \\
28.2 \\
28.2 \\
28.6 \\
29.7\end{array}$ & $\begin{array}{l}31.3 \\
28.6 \\
28.8 \\
30.1 \\
30.6\end{array}$ & $\begin{array}{l}31.7 \\
31.5 \\
31.7 \\
31.2 \\
30.8\end{array}$ & $\begin{array}{l}30.0 \\
30.0 \\
30.2 \\
29.5 \\
28.3\end{array}$ & $\begin{array}{l}31.1 \\
31.0 \\
31.0 \\
30.3 \\
29.1\end{array}$ & $\begin{array}{l}23.8 \\
23.3 \\
23.0 \\
23.2 \\
23.8\end{array}$ & $\begin{array}{l}21.5 \\
22.0 \\
22.0 \\
21.8 \\
22.3\end{array}$ & $\begin{array}{l}22.8 \\
22.5 \\
22.6 \\
22.4 \\
23.1\end{array}$ \\
\hline $\begin{array}{l}11 \\
12 \\
13 \\
14 \\
15\end{array}$ & $\begin{array}{l}31.9 \\
31.4 \\
32.8 \\
33.1 \\
33.1\end{array}$ & $\begin{array}{l}28.6 \\
27.5 \\
30.6 \\
32.0 \\
32.2\end{array}$ & $\begin{array}{l}31.0 \\
29.5 \\
31.8 \\
32.6 \\
32.8\end{array}$ & $\begin{array}{l}29.0 \\
29.0 \\
28.8 \\
29.3 \\
28.4\end{array}$ & $\begin{array}{l}27.5 \\
27.9 \\
27.7 \\
27.9 \\
27.3\end{array}$ & $\begin{array}{l}28.5 \\
28.6 \\
28.3 \\
28.5 \\
27.8\end{array}$ & $\begin{array}{l}23.7 \\
23.3 \\
23.7 \\
23.5 \\
22.3\end{array}$ & $\begin{array}{l}22.5 \\
22.3 \\
22.4 \\
22.3 \\
19.8\end{array}$ & $\begin{array}{l}23.1 \\
22.8 \\
23.1 \\
23.1 \\
20.8\end{array}$ \\
\hline $\begin{array}{l}16 \\
17 \\
18 \\
19 \\
20\end{array}$ & $\begin{array}{l}33.9 \\
33.4 \\
33.2 \\
33.1 \\
33.6\end{array}$ & $\begin{array}{l}32.1 \\
31.9 \\
32.0 \\
31.8 \\
31.4\end{array}$ & $\begin{array}{l}33.0 \\
32.8 \\
32.7 \\
32.6 \\
32.6\end{array}$ & $\begin{array}{l}27.6 \\
27.2 \\
27.2 \\
26.5 \\
25.7\end{array}$ & $\begin{array}{l}26.1 \\
25.8 \\
25.8 \\
25.1 \\
24.8\end{array}$ & $\begin{array}{l}26.9 \\
26.6 \\
26.3 \\
25.7 \\
25.3\end{array}$ & $\begin{array}{l}21.3 \\
20.6 \\
20.2 \\
21.3 \\
20.3\end{array}$ & $\begin{array}{l}20.2 \\
19.0 \\
19.1 \\
19.3 \\
18.5\end{array}$ & $\begin{array}{l}20.7 \\
19.8 \\
19.7 \\
20.4 \\
19.3\end{array}$ \\
\hline $\begin{array}{l}21 \\
22 \\
23 \\
24 \\
25\end{array}$ & $\begin{array}{l}34.5 \\
35.6 \\
34.5 \\
34.7 \\
34.1\end{array}$ & $\begin{array}{l}32.6 \\
33.8 \\
32.5 \\
32.1 \\
32.5\end{array}$ & $\begin{array}{l}33.6 \\
34.7 \\
33.4 \\
33.4 \\
33.3\end{array}$ & $\begin{array}{l}26.1 \\
25.6 \\
25.1 \\
24.8 \\
24.6\end{array}$ & $\begin{array}{l}25.1 \\
24.8 \\
23.8 \\
23.8 \\
23.8\end{array}$ & $\begin{array}{l}25.7 \\
25.2 \\
24.6 \\
24.2 \\
24.3\end{array}$ & $\begin{array}{l}19.1 \\
18.6 \\
18.9 \\
18.7 \\
18.0\end{array}$ & $\begin{array}{l}17.9 \\
17.0 \\
16.1 \\
17.0 \\
15.4\end{array}$ & $\begin{array}{l}18.6 \\
18.2 \\
17.3 \\
17.8 \\
16.7\end{array}$ \\
\hline $\begin{array}{l}26 \\
27 \\
28 \\
29 \\
30 \\
31\end{array}$ & $\begin{array}{l}33.7 \\
33.9 \\
33.9 \\
34.5 \\
34.0 \\
33.8\end{array}$ & $\begin{array}{l}31.9 \\
31.6 \\
33.1 \\
32.1 \\
32.5 \\
31.6\end{array}$ & $\begin{array}{l}32.9 \\
32.8 \\
33.6 \\
33.4 \\
33.2 \\
32.7\end{array}$ & $\begin{array}{l}24.8 \\
24.9 \\
25.6 \\
25.1 \\
24.5\end{array}$ & $\begin{array}{l}23.5 \\
23.8 \\
24.1 \\
23.8 \\
23.5\end{array}$ & $\begin{array}{l}24.2 \\
24.4 \\
24.8 \\
24.5 \\
24.0\end{array}$ & $\begin{array}{l}17.0 \\
15.8 \\
16.1 \\
16.5 \\
16.9 \\
15.4\end{array}$ & $\begin{array}{l}15.1 \\
15.1 \\
14.9 \\
14.6 \\
15.4 \\
13.8\end{array}$ & $\begin{array}{l}15.7 \\
15.4 \\
15.2 \\
15.2 \\
16.3 \\
14.3\end{array}$ \\
\hline Month & 35.6 & 27.5 & 32.4 & 33.8 & 23.5 & 27.7 & 25.8 & 13.8 & 20.3 \\
\hline
\end{tabular}


Table 26. Daily maximum, minimum, and mean dissolved oxygen concentrations at station 403155080373501, from the New Cumberland Dam (upstream) continuous recording water-quality monitor, June to october 1995

[--, value not determined]

Dissolved oxygen concentration, in milligrams per liter

\begin{tabular}{ccccc} 
May & \multicolumn{1}{c}{ June } & July \\
\cline { 2 - 4 } & Maximum Minimum Mean & Maximum Minimum Mean & Maximum Minimum Mean \\
\hline
\end{tabular}

\begin{tabular}{|c|c|c|c|c|c|c|c|c|c|}
\hline $\begin{array}{l}1 \\
2 \\
3 \\
4 \\
5\end{array}$ & $\begin{array}{l}- \\
- \\
- \\
- \\
- \\
- \\
-\end{array}$ & $\begin{array}{l}\ldots \\
\cdots \\
\cdots \\
\cdots\end{array}$ & $\begin{array}{l}\cdots \\
\cdots \\
\cdots \\
\cdots \\
\cdots\end{array}$ & $\begin{array}{l}\cdots \\
\cdots \\
\cdots \\
\cdots \\
\cdots\end{array}$ & $\begin{array}{l}\cdots \\
\cdots \\
\cdots \\
\cdots \\
\cdots\end{array}$ & $\begin{array}{l}\cdots \\
\cdots \\
\cdots \\
\cdots \\
\cdots\end{array}$ & $\begin{array}{l}7.6 \\
7.7 \\
8.3 \\
8.2 \\
7.9\end{array}$ & $\begin{array}{l}7.1 \\
7.5 \\
7.6 \\
7.7 \\
7.6\end{array}$ & $\begin{array}{l}7.4 \\
7.6 \\
7.9 \\
7.9 \\
7.7\end{array}$ \\
\hline $\begin{array}{r}6 \\
7 \\
8 \\
9 \\
10\end{array}$ & $\begin{array}{l}-- \\
\cdots \\
-- \\
-- \\
--\end{array}$ & $\begin{array}{l}\cdots \\
\cdots \\
\cdots \\
\cdots\end{array}$ & $\begin{array}{l}\cdots \\
\cdots \\
\cdots \\
\cdots \\
\cdots\end{array}$ & $\begin{array}{l}\cdots \\
- \\
\cdots \\
\cdots \\
\cdots\end{array}$ & $\begin{array}{l}\ldots \\
\cdots \\
\cdots \\
\cdots \\
\cdots\end{array}$ & $\begin{array}{l}\cdots \\
\cdots \\
\cdots \\
\cdots \\
\cdots\end{array}$ & $\begin{array}{l}8.0 \\
8.3 \\
8.8 \\
8.1 \\
7.8\end{array}$ & $\begin{array}{l}7.8 \\
8.0 \\
8.1 \\
7.8 \\
7.4\end{array}$ & $\begin{array}{l}7.9 \\
8.1 \\
8.4 \\
7.9 \\
7.6\end{array}$ \\
\hline $\begin{array}{l}11 \\
12 \\
13 \\
14 \\
15\end{array}$ & $\begin{array}{l}- \\
- \\
- \\
- \\
- \\
-\end{array}$ & $\begin{array}{c}\cdots \\
\cdots \\
\cdots \\
\cdots\end{array}$ & $\begin{array}{l}\cdots \\
\cdots \\
\cdots \\
\cdots \\
\cdots\end{array}$ & $\begin{array}{l}\cdots \\
\cdots \\
\cdots \\
- \\
\cdots\end{array}$ & $\begin{array}{l}\cdots \\
\cdots \\
\cdots \\
\cdots \\
\cdots\end{array}$ & $\begin{array}{l}\ldots \\
\ldots \\
\cdots \\
\cdots \\
\cdots\end{array}$ & $\begin{array}{l}7.5 \\
7.7 \\
7.9 \\
7.7 \\
7.6\end{array}$ & $\begin{array}{l}7.4 \\
7.5 \\
7.5 \\
7.5 \\
7.4\end{array}$ & $\begin{array}{l}7.4 \\
7.6 \\
7.7 \\
7.6 \\
7.5\end{array}$ \\
\hline $\begin{array}{l}16 \\
17 \\
18 \\
19 \\
20\end{array}$ & $\begin{array}{l}\cdots \\
-- \\
- \\
- \\
\cdots\end{array}$ & $\begin{array}{c}\cdots \\
\cdots \\
\cdots \\
\cdots \\
\cdots\end{array}$ & $\begin{array}{l}\cdots \\
\cdots \\
\cdots \\
\cdots \\
\cdots\end{array}$ & $\begin{array}{r}9.4 \\
10.7 \\
10.1 \\
9.8\end{array}$ & $\begin{array}{l}-.- \\
8.7 \\
8.6 \\
9.3 \\
8.6\end{array}$ & $\begin{array}{l}-. . \\
9.0 \\
9.5 \\
9.7 \\
9.1\end{array}$ & $\begin{array}{l}7.7 \\
7.4 \\
7.3 \\
7.7 \\
7.2\end{array}$ & $\begin{array}{l}7.4 \\
6.9 \\
6.7 \\
7.0 \\
6.9\end{array}$ & $\begin{array}{l}7.5 \\
7.1 \\
7.0 \\
7.2 \\
7.0\end{array}$ \\
\hline $\begin{array}{l}21 \\
22 \\
23 \\
24 \\
25\end{array}$ & $\begin{array}{l}\cdots \\
\cdots \\
\cdots \\
\cdots\end{array}$ & $\begin{array}{l}\cdots \\
\cdots \\
\cdots \\
\cdots\end{array}$ & $\begin{array}{l}\cdots \\
\cdots \\
\cdots \\
\cdots \\
-\end{array}$ & $\begin{array}{l}9.0 \\
8.0 \\
7.3 \\
7.1 \\
7.4\end{array}$ & $\begin{array}{l}8.0 \\
7.3 \\
6.9 \\
6.8 \\
7.0\end{array}$ & $\begin{array}{l}8.7 \\
7.7 \\
7.1 \\
7.0 \\
7.2\end{array}$ & $\begin{array}{l}7.1 \\
6.9 \\
7.0 \\
7.0 \\
7.0\end{array}$ & $\begin{array}{l}6.7 \\
6.8 \\
6.9 \\
6.8 \\
6.8\end{array}$ & $\begin{array}{l}6.9 \\
6.9 \\
6.9 \\
6.8 \\
6.9\end{array}$ \\
\hline $\begin{array}{l}26 \\
27 \\
28 \\
29 \\
30 \\
31\end{array}$ & $\begin{array}{l}-\cdots \\
\cdots \\
\cdots \\
\cdots \\
\cdots \\
-\cdots\end{array}$ & $\begin{array}{l}\cdots \\
\cdots \\
\cdots \\
\cdots \\
\cdots\end{array}$ & $\begin{array}{l}\ldots \\
- \\
-\ldots \\
-\ldots \\
\ldots \\
\cdots\end{array}$ & $\begin{array}{l}7.6 \\
7.2 \\
7.3 \\
7.2 \\
7.3 \\
-. .\end{array}$ & $\begin{array}{l}7.0 \\
6.9 \\
7.0 \\
6.8 \\
7.0 \\
\ldots-.\end{array}$ & $\begin{array}{l}7.2 \\
7.0 \\
7.1 \\
7.0 \\
7.2 \\
-.-\end{array}$ & $\begin{array}{l}7.2 \\
7.9 \\
8.1 \\
9.0 \\
8.6 \\
8.4\end{array}$ & $\begin{array}{l}6.9 \\
7.1 \\
7.9 \\
7.9 \\
8.1 \\
8.1\end{array}$ & $\begin{array}{l}7.0 \\
7.4 \\
8.0 \\
8.3 \\
8.3 \\
8.2\end{array}$ \\
\hline Month & $\cdots$ & $\cdots$ & $\cdots$ & $\cdots$ & $\cdots$ & $\cdots$ & 9.0 & 6.7 & 7.5 \\
\hline
\end{tabular}


Table 26. Daily maximum, minimum, and mean dissolved oxygen concentrations at station 403155080373501, from the New Cumberland Dam (upstream) continuous recording water-quality monitor, June to october 1995, Continued.

[.-, value not determined]

\begin{tabular}{|c|c|c|c|c|c|c|c|c|c|}
\hline \multirow{3}{*}{ Day } & \multicolumn{9}{|c|}{ Dissolved oxygen cc } \\
\hline & \multicolumn{3}{|c|}{ August } & \multicolumn{3}{|c|}{ September } & \multicolumn{3}{|c|}{ October } \\
\hline & Maximum & Minimum & Mean & Maximum & Minimum & Mean & Maximum & Minimum & Mean \\
\hline $\begin{array}{l}1 \\
2 \\
3 \\
4 \\
5\end{array}$ & $\begin{array}{l}8.4 \\
8.5 \\
8.0 \\
8.0 \\
7.1\end{array}$ & $\begin{array}{l}8.1 \\
7.8 \\
7.8 \\
7.1 \\
6.8\end{array}$ & $\begin{array}{l}8.3 \\
8.0 \\
7.9 \\
7.6 \\
6.9\end{array}$ & $\begin{array}{l}7.1 \\
7.0 \\
7.1 \\
7.5 \\
7.5\end{array}$ & $\begin{array}{l}6.7 \\
6.4 \\
6.3 \\
6.6 \\
6.9\end{array}$ & $\begin{array}{l}6.9 \\
6.8 \\
6.7 \\
7.0 \\
7.2\end{array}$ & $\begin{array}{l}8.3 \\
8.3 \\
8.6 \\
8.6 \\
8.3\end{array}$ & $\begin{array}{l}8.1 \\
8.1 \\
8.2 \\
8.3 \\
8.1\end{array}$ & $\begin{array}{l}8.2 \\
8.2 \\
8.4 \\
8.4 \\
8.2\end{array}$ \\
\hline $\begin{array}{r}6 \\
7 \\
8 \\
9 \\
10\end{array}$ & $\begin{array}{l}6.8 \\
7.0 \\
7.7 \\
7.7 \\
8.0\end{array}$ & $\begin{array}{l}6.2 \\
6.0 \\
7.0 \\
7.4 \\
7.4\end{array}$ & $\begin{array}{l}6.4 \\
6.4 \\
7.3 \\
7.5 \\
7.7\end{array}$ & $\begin{array}{l}7.7 \\
7.4 \\
7.7 \\
7.5 \\
8.0\end{array}$ & $\begin{array}{l}6.8 \\
6.8 \\
7.3 \\
7.1 \\
7.2\end{array}$ & $\begin{array}{l}7.2 \\
7.0 \\
7.4 \\
7.3 \\
7.5\end{array}$ & $\begin{array}{l}8.2 \\
8.1 \\
8.5 \\
8.4 \\
8.5\end{array}$ & $\begin{array}{l}8.1 \\
8.0 \\
8.1 \\
8.3 \\
8.3\end{array}$ & $\begin{array}{l}8.2 \\
8.1 \\
8.3 \\
8.4 \\
8.4\end{array}$ \\
\hline $\begin{array}{l}11 \\
12 \\
13 \\
14 \\
15\end{array}$ & $\begin{array}{l}8.4 \\
8.5 \\
8.1 \\
8.1 \\
8.3\end{array}$ & $\begin{array}{l}7.6 \\
7.7 \\
7.5 \\
7.7 \\
7.8\end{array}$ & $\begin{array}{l}7.9 \\
8.2 \\
7.7 \\
7.9 \\
8.1\end{array}$ & $\begin{array}{l}7.4 \\
7.0 \\
7.2 \\
8.0 \\
7.6\end{array}$ & $\begin{array}{l}7.0 \\
6.9 \\
6.9 \\
6.9 \\
7.1\end{array}$ & $\begin{array}{l}7.2 \\
7.0 \\
7.0 \\
7.3 \\
7.3\end{array}$ & $\begin{array}{l}8.7 \\
8.6 \\
8.6 \\
8.6 \\
8.4\end{array}$ & $\begin{array}{l}8.4 \\
8.4 \\
8.5 \\
8.3 \\
8.2\end{array}$ & $\begin{array}{l}8.5 \\
8.5 \\
8.5 \\
8.4 \\
8.3\end{array}$ \\
\hline $\begin{array}{l}16 \\
17 \\
18 \\
19 \\
20\end{array}$ & $\begin{array}{l}9.1 \\
8.9 \\
8.3 \\
7.9 \\
7.7\end{array}$ & $\begin{array}{l}8.0 \\
8.1 \\
7.8 \\
7.6 \\
7.6\end{array}$ & $\begin{array}{l}8.3 \\
8.4 \\
8.0 \\
7.7 \\
7.6\end{array}$ & $\begin{array}{l}7.1 \\
7.0 \\
7.0 \\
6.9 \\
7.1\end{array}$ & $\begin{array}{l}6.9 \\
6.9 \\
6.8 \\
6.7 \\
6.9\end{array}$ & $\begin{array}{l}7.0 \\
7.0 \\
6.9 \\
6.8 \\
7.0\end{array}$ & $\begin{array}{l}8.3 \\
8.4 \\
8.6 \\
8.8 \\
9.0\end{array}$ & $\begin{array}{l}8.2 \\
8.1 \\
8.4 \\
8.6 \\
8.8\end{array}$ & $\begin{array}{l}8.3 \\
8.3 \\
8.5 \\
8.7 \\
9.0\end{array}$ \\
\hline $\begin{array}{l}21 \\
22 \\
23 \\
24 \\
25\end{array}$ & $\begin{array}{l}7.6 \\
7.8 \\
7.8 \\
7.4 \\
7.8\end{array}$ & $\begin{array}{l}7.3 \\
7.1 \\
7.1 \\
7.1 \\
7.3\end{array}$ & $\begin{array}{l}7.5 \\
7.3 \\
7.5 \\
7.2 \\
7.5\end{array}$ & $\begin{array}{l}6.9 \\
6.9 \\
7.1 \\
7.1 \\
7.3\end{array}$ & $\begin{array}{l}6.8 \\
6.7 \\
6.7 \\
6.8 \\
7.1\end{array}$ & $\begin{array}{l}6.9 \\
6.8 \\
6.8 \\
7.0 \\
7.2\end{array}$ & $\begin{array}{l}9.2 \\
9.2 \\
9.5 \\
9.5 \\
9.5\end{array}$ & $\begin{array}{l}9.0 \\
9.1 \\
9.1 \\
9.2 \\
9.3\end{array}$ & $\begin{array}{l}9.1 \\
9.2 \\
9.3 \\
9.3 \\
9.4\end{array}$ \\
\hline $\begin{array}{l}26 \\
27 \\
28 \\
29 \\
30 \\
31\end{array}$ & $\begin{array}{l}7.6 \\
8.1 \\
8.2 \\
7.7 \\
7.6 \\
7.2\end{array}$ & $\begin{array}{l}7.3 \\
7.4 \\
7.5 \\
7.3 \\
7.2 \\
6.8\end{array}$ & $\begin{array}{l}7.4 \\
7.8 \\
7.9 \\
7.5 \\
7.4 \\
7.0\end{array}$ & $\begin{array}{l}7.4 \\
7.7 \\
8.1 \\
8.4 \\
8.3 \\
\ldots .\end{array}$ & $\begin{array}{l}7.1 \\
7.4 \\
7.6 \\
8.0 \\
8.1 \\
\ldots .-\end{array}$ & $\begin{array}{l}7.3 \\
7.5 \\
7.9 \\
8.2 \\
8.2 \\
-.-\end{array}$ & $\begin{array}{l}9.9 \\
9.8 \\
9.8 \\
9.9 \\
9.8 \\
9.9\end{array}$ & $\begin{array}{l}9.4 \\
9.6 \\
9.6 \\
9.7 \\
9.6 \\
9.6\end{array}$ & $\begin{array}{l}9.6 \\
9.7 \\
9.7 \\
9.8 \\
9.7 \\
9.7\end{array}$ \\
\hline Month & 9.1 & 6.0 & 7.6 & 8.4 & 6.3 & 7.2 & 9.9 & 8.0 & 8.8 \\
\hline
\end{tabular}


Table 27. Daily maximum, minimum, and mean specific conductance at station 403133080372801, from the New Cumberland Dam (downstream) continuous-recording water-quality monitor, June to October 1995

[.., value not determined]

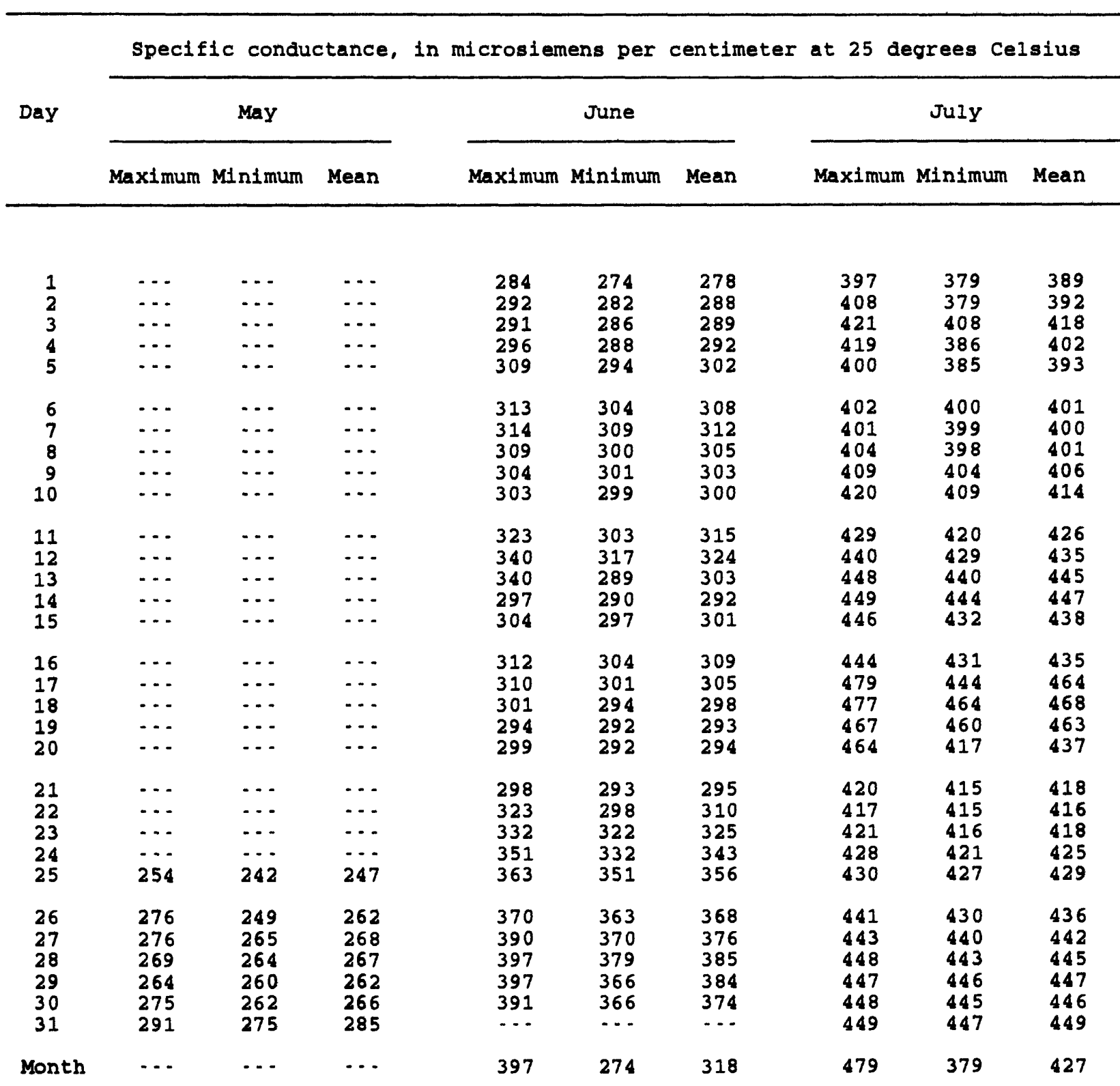


Table 27. Daily maximum, minimum, and mean specific conductance at station 403133080372801 , from the New Cumberland Dam (downstream) continuous-recording water-quality monitor, June to october 1995, Continued.

[.., value not determined]

\begin{tabular}{|c|c|c|c|c|c|c|c|c|c|}
\hline \multirow{3}{*}{ Day } & \multicolumn{3}{|c|}{ specific conductance, } & \multirow{2}{*}{\multicolumn{3}{|c|}{$\begin{array}{l}\text { mens per centimeter } \\
\text { September }\end{array}$}} & \multirow{2}{*}{\multicolumn{3}{|c|}{$\frac{\text { at } 25 \text { degrees Celsius }}{\text { October }}$}} \\
\hline & \multicolumn{3}{|c|}{ August } & & & & & & \\
\hline & Maximum & Minimum & Mean & Maximum & Minimum & Mean & Maximum & Minimum & Mean \\
\hline $\begin{array}{l}1 \\
2 \\
3 \\
4 \\
5\end{array}$ & $\begin{array}{l}454 \\
460 \\
470 \\
478 \\
478\end{array}$ & $\begin{array}{l}448 \\
452 \\
460 \\
470 \\
475\end{array}$ & $\begin{array}{l}451 \\
456 \\
466 \\
475 \\
477\end{array}$ & $\begin{array}{l}464 \\
463 \\
460 \\
460 \\
461\end{array}$ & $\begin{array}{l}462 \\
460 \\
459 \\
458 \\
458\end{array}$ & $\begin{array}{l}463 \\
461 \\
459 \\
459 \\
459\end{array}$ & $\begin{array}{l}480 \\
476 \\
478 \\
478 \\
496\end{array}$ & $\begin{array}{l}474 \\
474 \\
468 \\
473 \\
477\end{array}$ & $\begin{array}{l}476 \\
475 \\
475 \\
476 \\
483\end{array}$ \\
\hline $\begin{array}{r}6 \\
7 \\
8 \\
9 \\
10\end{array}$ & $\begin{array}{l}477 \\
468 \\
462 \\
463 \\
467\end{array}$ & $\begin{array}{l}468 \\
462 \\
450 \\
451 \\
463\end{array}$ & $\begin{array}{l}475 \\
465 \\
453 \\
457 \\
465\end{array}$ & $\begin{array}{l}469 \\
484 \\
488 \\
491 \\
496\end{array}$ & $\begin{array}{l}460 \\
468 \\
484 \\
487 \\
491\end{array}$ & $\begin{array}{l}464 \\
474 \\
486 \\
490 \\
493\end{array}$ & $\begin{array}{l}512 \\
515 \\
510 \\
510 \\
514\end{array}$ & $\begin{array}{l}496 \\
510 \\
505 \\
505 \\
510\end{array}$ & $\begin{array}{l}507 \\
513 \\
507 \\
507 \\
512\end{array}$ \\
\hline $\begin{array}{l}11 \\
12 \\
13 \\
14 \\
15\end{array}$ & $\begin{array}{l}490 \\
541 \\
551 \\
551 \\
538\end{array}$ & $\begin{array}{l}467 \\
490 \\
539 \\
538 \\
526\end{array}$ & $\begin{array}{l}472 \\
527 \\
547 \\
545 \\
531\end{array}$ & $\begin{array}{l}500 \\
500 \\
499 \\
494 \\
497\end{array}$ & $\begin{array}{l}496 \\
497 \\
493 \\
492 \\
493\end{array}$ & $\begin{array}{l}498 \\
499 \\
497 \\
493 \\
494\end{array}$ & $\begin{array}{l}517 \\
516 \\
512 \\
509 \\
515\end{array}$ & $\begin{array}{l}514 \\
512 \\
508 \\
507 \\
508\end{array}$ & $\begin{array}{l}516 \\
515 \\
509 \\
508 \\
511\end{array}$ \\
\hline $\begin{array}{l}16 \\
17 \\
18 \\
19 \\
20\end{array}$ & $\begin{array}{l}527 \\
520 \\
507 \\
490 \\
479\end{array}$ & $\begin{array}{l}520 \\
506 \\
490 \\
478 \\
476\end{array}$ & $\begin{array}{l}523 \\
513 \\
499 \\
484 \\
477\end{array}$ & $\begin{array}{l}499 \\
501 \\
501 \\
499 \\
496\end{array}$ & $\begin{array}{l}496 \\
498 \\
499 \\
496 \\
494\end{array}$ & $\begin{array}{l}498 \\
499 \\
500 \\
497 \\
495\end{array}$ & $\begin{array}{l}517 \\
512 \\
484 \\
461 \\
474\end{array}$ & $\begin{array}{l}512 \\
483 \\
457 \\
456 \\
461\end{array}$ & $\begin{array}{l}515 \\
501 \\
464 \\
458 \\
466\end{array}$ \\
\hline $\begin{array}{l}21 \\
22 \\
23 \\
24 \\
25\end{array}$ & $\begin{array}{l}480 \\
476 \\
475 \\
470 \\
470\end{array}$ & $\begin{array}{l}476 \\
472 \\
469 \\
468 \\
468\end{array}$ & $\begin{array}{l}478 \\
475 \\
472 \\
469 \\
469\end{array}$ & $\begin{array}{l}495 \\
495 \\
494 \\
494 \\
491\end{array}$ & $\begin{array}{l}491 \\
489 \\
492 \\
490 \\
488\end{array}$ & $\begin{array}{l}493 \\
491 \\
493 \\
492 \\
489\end{array}$ & $\begin{array}{l}488 \\
489 \\
484 \\
491 \\
496\end{array}$ & $\begin{array}{l}474 \\
484 \\
476 \\
478 \\
491\end{array}$ & $\begin{array}{l}482 \\
488 \\
479 \\
484 \\
495\end{array}$ \\
\hline $\begin{array}{l}26 \\
27 \\
28 \\
29 \\
30 \\
31\end{array}$ & $\begin{array}{l}473 \\
473 \\
473 \\
471 \\
471 \\
472\end{array}$ & $\begin{array}{l}470 \\
471 \\
469 \\
469 \\
469 \\
464\end{array}$ & $\begin{array}{l}471 \\
472 \\
470 \\
470 \\
470 \\
467\end{array}$ & $\begin{array}{l}489 \\
489 \\
486 \\
486 \\
485 \\
---\end{array}$ & $\begin{array}{l}487 \\
485 \\
485 \\
484 \\
479 \\
\cdots-\end{array}$ & $\begin{array}{l}488 \\
487 \\
485 \\
485 \\
482 \\
---\end{array}$ & $\begin{array}{l}496 \\
494 \\
489 \\
493 \\
497 \\
497\end{array}$ & $\begin{array}{l}493 \\
488 \\
487 \\
485 \\
492 \\
476\end{array}$ & $\begin{array}{l}494 \\
491 \\
488 \\
489 \\
496 \\
487\end{array}$ \\
\hline Month & 551 & 448 & 482 & 501 & 458 & 485 & 517 & 456 & 492 \\
\hline
\end{tabular}


Table 28. Daily maximum, minimum, and median $p H$ at station 403133080372801, from the New Cumberland Dam (downstream) continuous-recording water-quality monitor, June to October 1995

[ - . value not determined]

$\mathrm{pH}$, in standard units

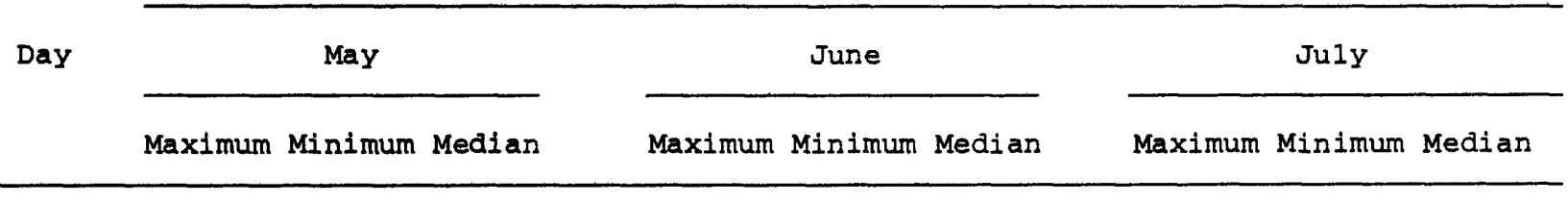

\begin{tabular}{|c|c|c|c|c|c|c|c|c|c|}
\hline $\begin{array}{l}1 \\
2 \\
3 \\
4 \\
5\end{array}$ & $\begin{array}{l}\cdots \\
\cdots \\
\cdots \\
\cdots \\
\cdots\end{array}$ & $\begin{array}{l}\cdots \\
\cdots \\
\cdots \\
\cdots\end{array}$ & $\begin{array}{l}\cdots \\
\cdots \\
\cdots \\
\cdots\end{array}$ & $\begin{array}{l}7.5 \\
7.5 \\
7.5 \\
7.5 \\
7.5\end{array}$ & $\begin{array}{l}7.4 \\
7.4 \\
7.4 \\
7.4 \\
7.4\end{array}$ & $\begin{array}{l}7.4 \\
7.5 \\
7.5 \\
7.5 \\
7.5\end{array}$ & $\begin{array}{l}7.4 \\
7.4 \\
7.5 \\
7.5 \\
7.4\end{array}$ & $\begin{array}{l}7.3 \\
7.4 \\
7.4 \\
7.4 \\
7.4\end{array}$ & $\begin{array}{l}7.4 \\
7.4 \\
7.4 \\
7.4 \\
7.4\end{array}$ \\
\hline $\begin{array}{r}6 \\
7 \\
8 \\
9 \\
10\end{array}$ & $\begin{array}{l}\cdots \\
\cdots \\
\cdots \\
\cdots \\
\cdots\end{array}$ & $\begin{array}{l}\cdots \\
\cdots \\
\cdots \\
\cdots\end{array}$ & $\begin{array}{l}\cdots \\
\cdots \\
\cdots \\
\cdots\end{array}$ & $\begin{array}{l}7.5 \\
7.5 \\
7.6 \\
7.6 \\
7.6\end{array}$ & $\begin{array}{l}7.4 \\
7.4 \\
7.4 \\
7.5 \\
7.5\end{array}$ & $\begin{array}{l}7.4 \\
7.4 \\
7.5 \\
7.6 \\
7.6\end{array}$ & $\begin{array}{l}7.5 \\
7.5 \\
7.6 \\
7.5 \\
7.5\end{array}$ & $\begin{array}{l}7.4 \\
7.5 \\
7.5 \\
7.4 \\
7.4\end{array}$ & $\begin{array}{l}7.5 \\
7.5 \\
7.5 \\
7.5 \\
7.4\end{array}$ \\
\hline $\begin{array}{l}11 \\
12 \\
13 \\
14 \\
15\end{array}$ & $\begin{array}{l}\ldots \\
\ldots \\
\cdots \\
\cdots \\
\cdots\end{array}$ & $\begin{array}{l}\cdots \\
\cdots \\
\cdots \\
\cdots\end{array}$ & $\begin{array}{l}\cdots \\
\cdots \\
\cdots \\
\cdots \\
\cdots\end{array}$ & $\begin{array}{l}7.5 \\
7.5 \\
7.6 \\
8.0 \\
7.8\end{array}$ & $\begin{array}{l}7.4 \\
7.4 \\
7.3 \\
7.6 \\
7.6\end{array}$ & $\begin{array}{l}7.5 \\
7.5 \\
7.4 \\
7.7 \\
7.6\end{array}$ & $\begin{array}{l}7.5 \\
7.4 \\
7.5 \\
7.5 \\
7.5\end{array}$ & $\begin{array}{l}7.4 \\
7.3 \\
7.4 \\
7.4 \\
7.4\end{array}$ & $\begin{array}{l}7.4 \\
7.4 \\
7.5 \\
7.5 \\
7.5\end{array}$ \\
\hline $\begin{array}{l}16 \\
17 \\
18 \\
19 \\
20\end{array}$ & $\begin{array}{l}\ldots \\
\ldots \\
\cdots \\
\cdots \\
\cdots\end{array}$ & $\begin{array}{c}\cdots \\
\cdots \\
\cdots \\
\cdots \\
\cdots\end{array}$ & $\begin{array}{l}\cdots \\
\cdots \\
\cdots \\
\cdots \\
\cdots\end{array}$ & $\begin{array}{l}7.6 \\
7.7 \\
8.0 \\
8.0 \\
7.9\end{array}$ & $\begin{array}{l}7.5 \\
7.5 \\
7.6 \\
7.7 \\
7.6\end{array}$ & $\begin{array}{l}7.6 \\
7.6 \\
7.7 \\
7.9 \\
7.7\end{array}$ & $\begin{array}{l}7.5 \\
7.5 \\
7.5 \\
7.6 \\
7.5\end{array}$ & $\begin{array}{l}7.5 \\
7.4 \\
7.4 \\
7.4 \\
7.4\end{array}$ & $\begin{array}{l}7.5 \\
7.5 \\
7.5 \\
7.5 \\
7.4\end{array}$ \\
\hline $\begin{array}{l}21 \\
22 \\
23 \\
24 \\
25\end{array}$ & $\begin{array}{l}\ldots \\
\ldots \\
\ldots \\
\ldots \\
7.5\end{array}$ & $\begin{array}{l}\cdots \\
\cdots \\
\cdots \\
7.4\end{array}$ & $\begin{array}{c}\cdots \\
\cdots \\
\cdots \\
\cdots \\
7.5\end{array}$ & $\begin{array}{l}8.0 \\
7.7 \\
7.5 \\
7.4 \\
7.5\end{array}$ & $\begin{array}{l}7.7 \\
7.5 \\
7.4 \\
7.4 \\
7.4\end{array}$ & $\begin{array}{l}7.8 \\
7.6 \\
7.4 \\
7.4 \\
7.4\end{array}$ & $\begin{array}{l}7.4 \\
7.4 \\
7.4 \\
7.4 \\
7.4\end{array}$ & $\begin{array}{l}7.4 \\
7.4 \\
7.4 \\
7.4 \\
7.3\end{array}$ & $\begin{array}{l}7.4 \\
7.4 \\
7.4 \\
7.4 \\
7.4\end{array}$ \\
\hline $\begin{array}{l}26 \\
27 \\
28 \\
29 \\
30 \\
31\end{array}$ & $\begin{array}{l}7.5 \\
7.5 \\
7.5 \\
7.6 \\
7.6 \\
7.5\end{array}$ & $\begin{array}{l}7.4 \\
7.4 \\
7.5 \\
7.5 \\
7.4 \\
7.4\end{array}$ & $\begin{array}{l}7.5 \\
7.5 \\
7.5 \\
7.5 \\
7.5 \\
7.5\end{array}$ & $\begin{array}{l}7.4 \\
7.4 \\
7.3 \\
7.3 \\
7.4 \\
\cdots\end{array}$ & $\begin{array}{l}7.4 \\
7.3 \\
7.3 \\
7.3 \\
7.3 \\
\cdots\end{array}$ & $\begin{array}{l}7.4 \\
7.4 \\
7.3 \\
7.3 \\
7.3 \\
\cdots .\end{array}$ & $\begin{array}{l}7.4 \\
7.5 \\
7.6 \\
7.6 \\
7.6 \\
7.6\end{array}$ & $\begin{array}{l}7.3 \\
7.4 \\
7.5 \\
7.4 \\
7.4 \\
7.4\end{array}$ & $\begin{array}{l}7.4 \\
7.4 \\
7.6 \\
7.5 \\
7.5 \\
7.5\end{array}$ \\
\hline Month & $\ldots$ & $\ldots$ & $\ldots$ & 8.0 & 7.3 & $\cdots$ & 7.6 & 7.3 & $\ldots$ \\
\hline
\end{tabular}


Table 28. Daily maximum, minimum, and median $p H$ at station 403133080372801, from the New Cumberland Dam (downstream) continuous-recording water-quality monitor, June to October 1995, Continued.

$\mathrm{pH}$, in standard units

Day $\begin{gathered}\text { August } \\ \text { Maximum Minimum Median }\end{gathered} \frac{\text { September }}{\text { Maximum Minimum Median }}$

\begin{tabular}{|c|c|c|c|c|c|c|c|c|c|}
\hline $\begin{array}{l}1 \\
2 \\
3 \\
4 \\
5\end{array}$ & $\begin{array}{l}7.6 \\
7.6 \\
7.6 \\
7.5 \\
7.3\end{array}$ & $\begin{array}{l}7.5 \\
7.5 \\
7.4 \\
7.3 \\
7.3\end{array}$ & $\begin{array}{l}7.6 \\
7.5 \\
7.5 \\
7.5 \\
7.3\end{array}$ & $\begin{array}{l}7.9 \\
8.0 \\
8.1 \\
8.2 \\
8.2\end{array}$ & $\begin{array}{l}7.8 \\
7.8 \\
7.7 \\
7.8 \\
7.9\end{array}$ & $\begin{array}{l}7.9 \\
7.9 \\
7.8 \\
8.0 \\
8.0\end{array}$ & $\begin{array}{l}7.6 \\
7.5 \\
7.5 \\
7.4 \\
7.4\end{array}$ & $\begin{array}{l}7.5 \\
7.5 \\
7.4 \\
7.4 \\
7.4\end{array}$ & $\begin{array}{l}7.5 \\
7.5 \\
7.5 \\
7.4 \\
7.4\end{array}$ \\
\hline $\begin{array}{r}6 \\
7 \\
8 \\
9 \\
10\end{array}$ & $\begin{array}{l}7.3 \\
7.4 \\
7.5 \\
7.6 \\
7.5\end{array}$ & $\begin{array}{l}7.2 \\
7.2 \\
7.3 \\
7.4 \\
7.4\end{array}$ & $\begin{array}{l}7.2 \\
7.3 \\
7.5 \\
7.5 \\
7.4\end{array}$ & $\begin{array}{l}8.2 \\
8.0 \\
8.1 \\
8.0 \\
8.0\end{array}$ & $\begin{array}{l}7.9 \\
7.8 \\
7.9 \\
7.9 \\
7.8\end{array}$ & $\begin{array}{l}8.0 \\
7.9 \\
8.0 \\
8.0 \\
7.9\end{array}$ & $\begin{array}{l}7.5 \\
7.4 \\
7.4 \\
7.4 \\
7.4\end{array}$ & $\begin{array}{l}7.4 \\
7.4 \\
7.4 \\
7.4 \\
7.4\end{array}$ & $\begin{array}{l}7.4 \\
7.4 \\
7.4 \\
7.4 \\
7.4\end{array}$ \\
\hline $\begin{array}{l}11 \\
12 \\
13 \\
14 \\
15\end{array}$ & $\begin{array}{l}7.6 \\
7.7 \\
7.7 \\
7.6 \\
7.6\end{array}$ & $\begin{array}{l}7.4 \\
7.5 \\
7.6 \\
7.6 \\
7.5\end{array}$ & $\begin{array}{l}7.5 \\
7.7 \\
7.6 \\
7.6 \\
7.5\end{array}$ & $\begin{array}{l}7.9 \\
7.7 \\
7.8 \\
7.8 \\
7.8\end{array}$ & $\begin{array}{l}7.7 \\
7.7 \\
7.7 \\
7.7 \\
7.6\end{array}$ & $\begin{array}{l}7.8 \\
7.7 \\
7.8 \\
7.8 \\
7.6\end{array}$ & $\begin{array}{l}7.4 \\
7.4 \\
7.4 \\
7.4 \\
7.4\end{array}$ & $\begin{array}{l}7.4 \\
7.4 \\
7.4 \\
7.3 \\
7.3\end{array}$ & $\begin{array}{l}7.4 \\
7.4 \\
7.4 \\
7.4 \\
7.4\end{array}$ \\
\hline $\begin{array}{l}16 \\
17 \\
18 \\
19 \\
20\end{array}$ & $\begin{array}{l}7.6 \\
7.6 \\
7.6 \\
7.5 \\
7.6\end{array}$ & $\begin{array}{l}7.5 \\
7.5 \\
7.5 \\
7.4 \\
7.4\end{array}$ & $\begin{array}{l}7.6 \\
7.6 \\
7.5 \\
7.5 \\
7.5\end{array}$ & $\begin{array}{l}7.6 \\
7.6 \\
7.6 \\
7.5 \\
7.5\end{array}$ & $\begin{array}{l}7.5 \\
7.5 \\
7.5 \\
7.5 \\
7.4\end{array}$ & $\begin{array}{l}7.6 \\
7.6 \\
7.5 \\
7.5 \\
7.4\end{array}$ & $\begin{array}{l}7.4 \\
7.6 \\
7.6 \\
7.7 \\
7.7\end{array}$ & $\begin{array}{l}7.3 \\
7.3 \\
7.6 \\
7.6 \\
7.7\end{array}$ & $\begin{array}{l}7.3 \\
7.4 \\
7.6 \\
7.7 \\
7.7\end{array}$ \\
\hline $\begin{array}{l}21 \\
22 \\
23 \\
24 \\
25\end{array}$ & $\begin{array}{l}7.6 \\
7.7 \\
7.8 \\
7.7 \\
7.9\end{array}$ & $\begin{array}{l}7.4 \\
7.5 \\
7.6 \\
7.6 \\
7.6\end{array}$ & $\begin{array}{l}7.5 \\
7.6 \\
7.7 \\
7.6 \\
7.7\end{array}$ & $\begin{array}{l}7.4 \\
7.4 \\
7.4 \\
7.4 \\
7.4\end{array}$ & $\begin{array}{l}7.4 \\
7.4 \\
7.4 \\
7.4 \\
7.4\end{array}$ & $\begin{array}{l}7.4 \\
7.4 \\
7.4 \\
7.4 \\
7.4\end{array}$ & $\begin{array}{l}7.7 \\
7.7 \\
7.7 \\
7.8 \\
7.8\end{array}$ & $\begin{array}{l}7.7 \\
7.7 \\
7.7 \\
7.7 \\
7.8\end{array}$ & $\begin{array}{l}7.7 \\
7.7 \\
7.7 \\
7.8 \\
7.8\end{array}$ \\
\hline $\begin{array}{l}26 \\
27 \\
28 \\
29 \\
30 \\
31\end{array}$ & $\begin{array}{l}8.0 \\
8.0 \\
8.3 \\
8.0 \\
8.0 \\
8.0\end{array}$ & $\begin{array}{l}7.6 \\
7.8 \\
7.9 \\
7.8 \\
7.8 \\
7.7\end{array}$ & $\begin{array}{l}7.8 \\
7.9 \\
8.0 \\
7.9 \\
7.9 \\
7.8\end{array}$ & $\begin{array}{l}7.5 \\
7.5 \\
7.5 \\
7.5 \\
7.6 \\
\cdots . .\end{array}$ & $\begin{array}{l}7.4 \\
7.4 \\
7.4 \\
7.4 \\
7.4 \\
-. .\end{array}$ & $\begin{array}{l}7.4 \\
7.4 \\
7.5 \\
7.5 \\
7.5 \\
. .\end{array}$ & $\begin{array}{l}7.8 \\
7.9 \\
7.9 \\
7.8 \\
7.8 \\
7.8\end{array}$ & $\begin{array}{l}7.8 \\
7.8 \\
7.8 \\
7.8 \\
7.8 \\
7.8\end{array}$ & $\begin{array}{l}7.8 \\
7.9 \\
7.8 \\
7.8 \\
7.8 \\
7.8\end{array}$ \\
\hline Month & 8.3 & 7.2 & $\cdots$ & 8.2 & 7.4 & $\cdots$ & 7.9 & 7.3 & $\cdots$ \\
\hline
\end{tabular}


Table 29. Daily maximum, minimum, and mean water temperature at station 403133080372801, from the New Cumberland Dam (downstream) continuous-recording water-quality monitor, June to October 1995

\begin{tabular}{|c|c|c|c|c|c|c|c|c|c|}
\hline \multirow{3}{*}{ Day } & \multicolumn{9}{|c|}{ Water temperature, in degrees Celsius } \\
\hline & \multicolumn{3}{|c|}{ May } & \multicolumn{3}{|c|}{ June } & \multicolumn{3}{|c|}{ July } \\
\hline & Maximum & Minimum & Mean & Maximum & Minimum & Mean & Maximum & Minimum & Mean \\
\hline $\begin{array}{l}1 \\
2 \\
3 \\
4 \\
5\end{array}$ & $\begin{array}{l}\cdots \\
\cdots \\
\cdots \\
\cdots \\
\cdots\end{array}$ & $\begin{array}{l}\cdots \\
\cdots \\
\cdots \\
\cdots \\
\cdots\end{array}$ & $\begin{array}{l}\cdots \\
\cdots \\
\cdots \\
\cdots \\
\cdots\end{array}$ & $\begin{array}{l}20.7 \\
20.8 \\
21.2 \\
21.0 \\
21.9\end{array}$ & $\begin{array}{l}19.8 \\
19.6 \\
20.0 \\
20.3 \\
20.8\end{array}$ & $\begin{array}{l}20.3 \\
20.4 \\
20.7 \\
20.6 \\
21.3\end{array}$ & $\begin{array}{l}26.5 \\
26.4 \\
26.3 \\
26.1 \\
27.3\end{array}$ & $\begin{array}{l}25.9 \\
25.7 \\
25.6 \\
25.4 \\
25.5\end{array}$ & $\begin{array}{l}26.2 \\
25.9 \\
25.9 \\
25.7 \\
26.3\end{array}$ \\
\hline $\begin{array}{r}6 \\
7 \\
8 \\
9 \\
10\end{array}$ & $\begin{array}{l}\cdots \\
\cdots \\
\cdots \\
\cdots\end{array}$ & $\begin{array}{l}\cdots \\
\cdots \\
\cdots \\
\cdots \\
\cdots\end{array}$ & $\begin{array}{l}\cdots \\
\cdots \\
\cdots \\
\cdots \\
\cdots\end{array}$ & $\begin{array}{l}22.4 \\
22.9 \\
23.6 \\
23.8 \\
23.7\end{array}$ & $\begin{array}{l}21.1 \\
21.8 \\
22.5 \\
22.8 \\
22.9\end{array}$ & $\begin{array}{l}21.7 \\
22.4 \\
23.0 \\
23.3 \\
23.4\end{array}$ & $\begin{array}{l}27.5 \\
27.4 \\
26.9 \\
26.2 \\
27.5\end{array}$ & $\begin{array}{l}26.6 \\
26.7 \\
26.0 \\
25.7 \\
25.9\end{array}$ & $\begin{array}{l}27.2 \\
27.1 \\
26.6 \\
26.0 \\
26.7\end{array}$ \\
\hline $\begin{array}{l}11 \\
12 \\
13 \\
14 \\
15\end{array}$ & $\begin{array}{l}\cdots \\
\cdots \\
\cdots \\
\cdots\end{array}$ & $\begin{array}{l}\cdots \\
\cdots \\
\cdots \\
\cdots \\
\cdots\end{array}$ & $\begin{array}{l}\cdots \\
\cdots \\
\cdots \\
\cdots \\
\cdots\end{array}$ & $\begin{array}{l}24.3 \\
24.2 \\
23.4 \\
24.4 \\
24.6\end{array}$ & $\begin{array}{l}23.2 \\
23.0 \\
22.5 \\
22.7 \\
23.2\end{array}$ & $\begin{array}{l}23.8 \\
23.8 \\
23.0 \\
23.7 \\
23.9\end{array}$ & $\begin{array}{l}28.1 \\
28.3 \\
28.9 \\
29.2 \\
29.7\end{array}$ & $\begin{array}{l}26.8 \\
27.4 \\
27.7 \\
28.3 \\
28.8\end{array}$ & $\begin{array}{l}27.5 \\
28.0 \\
28.2 \\
28.8 \\
29.2\end{array}$ \\
\hline $\begin{array}{l}16 \\
17 \\
18 \\
19 \\
20\end{array}$ & $\begin{array}{l}\cdots \\
\cdots \\
\cdots \\
\cdots \\
\cdots\end{array}$ & $\begin{array}{l}\cdots \\
\cdots \\
\cdots \\
\cdots \\
\cdots\end{array}$ & $\begin{array}{l}\cdots \\
\cdots \\
\cdots \\
\cdots \\
\cdots\end{array}$ & $\begin{array}{l}24.6 \\
24.6 \\
25.1 \\
25.7 \\
26.3\end{array}$ & $\begin{array}{l}23.2 \\
23.4 \\
23.5 \\
24.6 \\
25.3\end{array}$ & $\begin{array}{l}23.9 \\
24.0 \\
24.2 \\
25.1 \\
25.8\end{array}$ & $\begin{array}{l}29.7 \\
29.1 \\
29.1 \\
29.2 \\
29.1\end{array}$ & $\begin{array}{l}28.7 \\
28.2 \\
28.7 \\
28.5 \\
28.5\end{array}$ & $\begin{array}{l}29.2 \\
28.6 \\
28.9 \\
28.9 \\
28.7\end{array}$ \\
\hline $\begin{array}{l}21 \\
22 \\
23 \\
24 \\
25\end{array}$ & $\begin{array}{c}\ldots \\
\cdots \\
\cdots \\
19.3\end{array}$ & $\begin{array}{c}\ldots \\
\ldots \\
\cdots \\
18.3\end{array}$ & $\begin{array}{c}\ldots \\
\cdots \\
\cdots \\
18.8\end{array}$ & $\begin{array}{l}26.5 \\
26.6 \\
26.7 \\
27.4 \\
27.3\end{array}$ & $\begin{array}{l}25.6 \\
25.9 \\
26.0 \\
26.3 \\
26.5\end{array}$ & $\begin{array}{l}26.1 \\
26.3 \\
26.3 \\
26.9 \\
26.9\end{array}$ & $\begin{array}{l}29.2 \\
29.5 \\
29.5 \\
29.6 \\
30.0\end{array}$ & $\begin{array}{l}28.3 \\
28.9 \\
29.1 \\
29.2 \\
29.3\end{array}$ & $\begin{array}{l}28.8 \\
29.1 \\
29.3 \\
29.4 \\
29.7\end{array}$ \\
\hline $\begin{array}{l}26 \\
27 \\
28 \\
29 \\
30 \\
31\end{array}$ & $\begin{array}{l}19.8 \\
19.3 \\
19.4 \\
19.6 \\
20.7 \\
20.7\end{array}$ & $\begin{array}{l}18.6 \\
18.6 \\
18.7 \\
18.9 \\
19.3 \\
19.3\end{array}$ & $\begin{array}{l}19.2 \\
19.0 \\
19.1 \\
19.2 \\
19.9 \\
20.1\end{array}$ & $\begin{array}{r}27.3 \\
27.4 \\
26.9 \\
26.9 \\
26.3 \\
\ldots\end{array}$ & $\begin{array}{r}26.5 \\
26.2 \\
25.8 \\
26.0 \\
25.5 \\
\ldots .-\end{array}$ & $\begin{array}{r}26.9 \\
26.7 \\
26.4 \\
26.4 \\
26.0 \\
\ldots\end{array}$ & $\begin{array}{l}30.0 \\
29.9 \\
29.8 \\
30.5 \\
30.7 \\
31.2\end{array}$ & $\begin{array}{l}29.6 \\
29.4 \\
29.4 \\
29.7 \\
29.9 \\
30.0\end{array}$ & $\begin{array}{l}29.8 \\
29.6 \\
29.6 \\
30.1 \\
30.3 \\
30.6\end{array}$ \\
\hline Month & $\cdots$ & $\ldots$ & $\cdots$ & 27.4 & 19.6 & 24.1 & 31.2 & 25.4 & 28.3 \\
\hline
\end{tabular}


Table 29. Daily maximum, minimum, and mean water temperature at station 403133080372801, from the New Cumberland Dam (downstream) continuous-recording water-quality monitor, June to October 1995, Continued.

$[\cdots$, value not determined]

\begin{tabular}{|c|c|c|c|c|c|c|c|c|c|}
\hline \multirow{3}{*}{ Day } & \multicolumn{9}{|c|}{ Water temperature, in degrees Celsius } \\
\hline & \multicolumn{3}{|c|}{ August } & \multicolumn{3}{|c|}{ September } & \multicolumn{3}{|c|}{ October } \\
\hline & Maximum & Minimum & Mean & Maximum & Minimum & Mean & Maximum & Minimum & Mean \\
\hline $\begin{array}{l}1 \\
2 \\
3 \\
4 \\
5\end{array}$ & $\begin{array}{l}31.1 \\
31.3 \\
31.7 \\
32.0 \\
32.0\end{array}$ & $\begin{array}{l}30.5 \\
30.5 \\
30.9 \\
31.5 \\
30.9\end{array}$ & $\begin{array}{l}30.8 \\
31.0 \\
31.3 \\
31.7 \\
31.6\end{array}$ & $\begin{array}{l}31.7 \\
31.7 \\
30.5 \\
30.3 \\
30.4\end{array}$ & $\begin{array}{l}31.1 \\
30.5 \\
29.7 \\
29.4 \\
29.2\end{array}$ & $\begin{array}{l}31.4 \\
30.9 \\
30.1 \\
29.9 \\
29.8\end{array}$ & $\begin{array}{l}23.4 \\
24.2 \\
24.1 \\
23.8 \\
23.2\end{array}$ & $\begin{array}{l}22.8 \\
23.0 \\
23.4 \\
22.6 \\
22.1\end{array}$ & $\begin{array}{l}23.1 \\
23.7 \\
23.7 \\
23.3 \\
22.5\end{array}$ \\
\hline $\begin{array}{r}6 \\
7 \\
8 \\
9 \\
10\end{array}$ & $\begin{array}{l}30.9 \\
30.8 \\
30.0 \\
29.9 \\
30.1\end{array}$ & $\begin{array}{l}29.9 \\
29.0 \\
29.5 \\
29.2 \\
29.5\end{array}$ & $\begin{array}{l}30.4 \\
30.0 \\
29.8 \\
29.6 \\
29.7\end{array}$ & $\begin{array}{l}30.1 \\
29.7 \\
29.8 \\
29.8 \\
28.7\end{array}$ & $\begin{array}{l}28.6 \\
28.8 \\
28.8 \\
28.5 \\
27.3\end{array}$ & $\begin{array}{l}29.4 \\
29.3 \\
29.3 \\
29.0 \\
27.7\end{array}$ & $\begin{array}{l}22.6 \\
22.3 \\
22.0 \\
21.8 \\
21.7\end{array}$ & $\begin{array}{l}21.5 \\
21.7 \\
21.2 \\
21.1 \\
21.1\end{array}$ & $\begin{array}{l}22.1 \\
22.0 \\
21.7 \\
21.5 \\
21.4\end{array}$ \\
\hline $\begin{array}{l}11 \\
12 \\
13 \\
14 \\
15\end{array}$ & $\begin{array}{l}30.3 \\
30.4 \\
31.0 \\
31.1 \\
31.2\end{array}$ & $\begin{array}{l}29.7 \\
29.0 \\
30.2 \\
30.7 \\
30.6\end{array}$ & $\begin{array}{l}30.0 \\
29.6 \\
30.6 \\
30.9 \\
30.9\end{array}$ & $\begin{array}{l}27.5 \\
26.9 \\
26.9 \\
27.0 \\
26.5\end{array}$ & $\begin{array}{l}26.5 \\
26.6 \\
25.9 \\
25.9 \\
25.6\end{array}$ & $\begin{array}{l}27.1 \\
26.8 \\
26.5 \\
26.4 \\
26.0\end{array}$ & $\begin{array}{l}22.1 \\
22.0 \\
22.5 \\
22.3 \\
21.6\end{array}$ & $\begin{array}{l}21.2 \\
21.3 \\
21.2 \\
21.6 \\
20.0\end{array}$ & $\begin{array}{l}21.7 \\
21.7 \\
21.8 \\
21.8 \\
20.4\end{array}$ \\
\hline $\begin{array}{l}16 \\
17 \\
18 \\
19 \\
20\end{array}$ & $\begin{array}{l}31.3 \\
31.4 \\
31.4 \\
31.5 \\
32.1\end{array}$ & $\begin{array}{l}30.7 \\
30.6 \\
30.9 \\
30.9 \\
30.6\end{array}$ & $\begin{array}{l}31.0 \\
31.0 \\
31.2 \\
31.1 \\
31.4\end{array}$ & $\begin{array}{l}25.6 \\
26.0 \\
26.0 \\
25.5 \\
24.8\end{array}$ & $\begin{array}{l}25.2 \\
25.2 \\
25.2 \\
24.7 \\
24.4\end{array}$ & $\begin{array}{l}25.4 \\
25.6 \\
25.6 \\
25.1 \\
24.7\end{array}$ & $\begin{array}{l}20.4 \\
19.9 \\
19.1 \\
19.2 \\
19.1\end{array}$ & $\begin{array}{l}19.6 \\
19.1 \\
18.6 \\
18.7 \\
18.4\end{array}$ & $\begin{array}{l}20.0 \\
19.5 \\
18.9 \\
18.9 \\
18.7\end{array}$ \\
\hline $\begin{array}{l}21 \\
22 \\
23 \\
24 \\
25\end{array}$ & $\begin{array}{l}32.5 \\
33.0 \\
32.3 \\
32.2 \\
32.0\end{array}$ & $\begin{array}{l}31.5 \\
32.3 \\
31.1 \\
30.8 \\
31.3\end{array}$ & $\begin{array}{l}32.1 \\
32.6 \\
31.5 \\
31.6 \\
31.6\end{array}$ & $\begin{array}{l}25.3 \\
24.8 \\
24.1 \\
23.4 \\
23.5\end{array}$ & $\begin{array}{l}24.8 \\
24.1 \\
23.0 \\
22.7 \\
22.8\end{array}$ & $\begin{array}{l}25.0 \\
24.4 \\
23.4 \\
23.0 \\
23.2\end{array}$ & $\begin{array}{l}18.6 \\
18.5 \\
17.8 \\
17.7 \\
17.5\end{array}$ & $\begin{array}{l}18.2 \\
17.7 \\
17.3 \\
17.1 \\
16.7\end{array}$ & $\begin{array}{l}18.4 \\
17.9 \\
17.5 \\
17.5 \\
16.9\end{array}$ \\
\hline $\begin{array}{l}26 \\
27 \\
28 \\
29 \\
30 \\
31\end{array}$ & $\begin{array}{l}31.9 \\
31.5 \\
32.0 \\
31.8 \\
31.6 \\
31.7\end{array}$ & $\begin{array}{l}30.9 \\
30.6 \\
30.9 \\
31.0 \\
30.8 \\
30.4\end{array}$ & $\begin{array}{l}31.4 \\
31.1 \\
31.5 \\
31.5 \\
31.2 \\
31.1\end{array}$ & $\begin{array}{r}23.6 \\
23.6 \\
24.0 \\
23.5 \\
23.7 \\
\ldots\end{array}$ & $\begin{array}{r}22.9 \\
22.7 \\
22.9 \\
22.8 \\
22.7 \\
\ldots .\end{array}$ & $\begin{array}{r}23.3 \\
23.2 \\
23.4 \\
23.1 \\
23.2 \\
\ldots . .\end{array}$ & $\begin{array}{l}16.8 \\
16.3 \\
16.4 \\
16.2 \\
16.3 \\
16.2\end{array}$ & $\begin{array}{l}16.1 \\
15.9 \\
15.8 \\
15.6 \\
15.5 \\
15.0\end{array}$ & $\begin{array}{l}16.4 \\
16.0 \\
16.1 \\
15.8 \\
16.0 \\
15.5\end{array}$ \\
\hline Month & 33.0 & 29.0 & 31.0 & 31.7 & 22.7 & 26.4 & 24.2 & 15.0 & 19.8 \\
\hline
\end{tabular}


Table 30. Daily maximum, minimum, and mean dissolved oxygen concentrations at station 403133080372801, from the New Cumberland Dam (downstream) continuous recording water-quality monitor, June to October 1995

$[\cdots$, value not determined]

\begin{tabular}{|c|c|c|c|c|c|c|c|c|c|}
\hline \multirow{3}{*}{ Day } & \multicolumn{9}{|c|}{ Dissolved oxygen cc } \\
\hline & \multicolumn{3}{|c|}{ May } & \multicolumn{3}{|c|}{ June } & \multicolumn{3}{|c|}{ July } \\
\hline & Maximum & Minimum & Mean & Maximum & Minimum & Mean & Maximum & Minimum & Mean \\
\hline $\begin{array}{l}1 \\
2 \\
3 \\
4 \\
5\end{array}$ & $\begin{array}{l}-- \\
-- \\
-- \\
-- \\
\cdots\end{array}$ & $\begin{array}{l}\cdots \\
\cdots \\
\cdots \\
\cdots \\
\cdots\end{array}$ & $\begin{array}{l}\cdots \\
\cdots \\
\cdots \\
\cdots \\
\cdots\end{array}$ & $\begin{array}{r}10.0 \\
10.0 \\
9.9 \\
10.0 \\
9.8\end{array}$ & $\begin{array}{l}9.9 \\
9.8 \\
9.8 \\
9.8 \\
9.5\end{array}$ & $\begin{array}{l}9.9 \\
9.9 \\
9.9 \\
9.9 \\
9.7\end{array}$ & $\begin{array}{l}\cdots \\
\cdots \\
\cdots \\
\cdots \\
\cdots\end{array}$ & $\begin{array}{l}\cdots \\
\cdots \\
\cdots \\
\cdots \\
\cdots\end{array}$ & $\begin{array}{l}\cdots \\
\cdots \\
\cdots \\
\cdots\end{array}$ \\
\hline $\begin{array}{r}6 \\
7 \\
8 \\
9 \\
10\end{array}$ & $\begin{array}{l}\cdots \\
\cdots \\
\cdots \\
\cdots \\
-\end{array}$ & $\begin{array}{l}\cdots \\
\cdots \\
\cdots \\
\cdots \\
\cdots\end{array}$ & $\begin{array}{l}\cdots \\
\cdots \\
\cdots \\
\cdots \\
\cdots\end{array}$ & $\begin{array}{l}9.6 \\
9.7 \\
9.7 \\
9.6 \\
.--\end{array}$ & $\begin{array}{l}9.5 \\
9.4 \\
9.4 \\
9.5 \\
\ldots .-\end{array}$ & $\begin{array}{l}9.5 \\
9.5 \\
9.6 \\
9.6 \\
-.-\end{array}$ & $\begin{array}{l}\cdots \\
\cdots \\
\cdots \\
\cdots \\
\cdots\end{array}$ & $\begin{array}{l}\cdots \\
\cdots \\
\cdots \\
\cdots \\
\cdots\end{array}$ & $\begin{array}{l}\cdots \\
\cdots \\
\cdots \\
\cdots\end{array}$ \\
\hline $\begin{array}{l}11 \\
12 \\
13 \\
14 \\
15\end{array}$ & $\begin{array}{l}\cdots \\
\cdots \\
\cdots \\
\cdots \\
\cdots\end{array}$ & $\begin{array}{l}\cdots \\
\cdots \\
\cdots \\
\cdots \\
\cdots\end{array}$ & $\begin{array}{l}\ldots \\
\cdots \\
\cdots \\
\cdots \\
\cdots\end{array}$ & $\begin{array}{l}\cdots \\
\cdots \\
\cdots \\
\cdots \\
\cdots\end{array}$ & $\begin{array}{l}\cdots \\
\cdots \\
\cdots \\
\cdots \\
\cdots\end{array}$ & $\begin{array}{l}\cdots \\
\cdots \\
\cdots \\
\cdots \\
\cdots\end{array}$ & $\begin{array}{l}-. . \\
8.4 \\
8.5 \\
8.3 \\
8.1\end{array}$ & $\begin{array}{l}7.6 \\
7.6 \\
8.0 \\
7.8 \\
7.7\end{array}$ & $\begin{array}{l}-.- \\
8.1 \\
8.3 \\
8.1 \\
7.9\end{array}$ \\
\hline $\begin{array}{l}16 \\
17 \\
18 \\
19 \\
20\end{array}$ & $\begin{array}{l}\cdots \\
\cdots \\
\cdots \\
\cdots \\
\cdots\end{array}$ & $\begin{array}{l}\cdots \\
\cdots \\
\cdots \\
\cdots\end{array}$ & $\begin{array}{l}\cdots \\
\cdots \\
\cdots \\
\cdots \\
\cdots\end{array}$ & $\begin{array}{l}\cdots \\
\cdots \\
\cdots \\
\cdots \\
\cdots\end{array}$ & $\begin{array}{l}\cdots \\
\cdots \\
\cdots \\
\cdots \\
\cdots\end{array}$ & $\begin{array}{l}-- \\
-- \\
\cdots \\
\cdots \\
\cdots\end{array}$ & $\begin{array}{l}8.1 \\
8.0 \\
7.9 \\
7.9 \\
7.7\end{array}$ & $\begin{array}{l}7.8 \\
7.6 \\
7.4 \\
7.4 \\
7.4\end{array}$ & $\begin{array}{l}8.0 \\
7.9 \\
7.7 \\
7.8 \\
7.6\end{array}$ \\
\hline $\begin{array}{l}21 \\
22 \\
23 \\
24 \\
25\end{array}$ & $\begin{array}{c}\cdots \\
\cdots \\
\cdots \\
10 . \\
10.3\end{array}$ & $\begin{array}{l}\ldots- \\
\cdots \\
\cdots \\
\cdots \\
9.8\end{array}$ & $\begin{array}{l}\ldots \\
\cdots \\
-\ldots \\
-\cdots \\
9.9\end{array}$ & $\begin{array}{l}-.- \\
8.6 \\
8.2 \\
7.9 \\
7.9\end{array}$ & $\begin{array}{l}-. . \\
8.1 \\
7.8 \\
7.6 \\
7.6\end{array}$ & $\begin{array}{l}\cdots \\
8.4 \\
8.1 \\
7.8 \\
7.7\end{array}$ & $\begin{array}{l}7.7 \\
7.6 \\
7.6 \\
7.6 \\
7.6\end{array}$ & $\begin{array}{l}7.4 \\
7.3 \\
7 \cdot 3 \\
7.2 \\
7.2\end{array}$ & $\begin{array}{l}7.6 \\
7.5 \\
7.5 \\
7.4 \\
7.4\end{array}$ \\
\hline $\begin{array}{l}26 \\
27 \\
28 \\
29 \\
30 \\
31\end{array}$ & $\begin{array}{l}10.2 \\
10.1 \\
10.1 \\
10.1 \\
10.1 \\
10.0\end{array}$ & $\begin{array}{r}10.0 \\
10.0 \\
9.9 \\
9.9 \\
9.8 \\
9.9\end{array}$ & $\begin{array}{r}10.1 \\
10.0 \\
10.0 \\
10.0 \\
9.9 \\
10.0\end{array}$ & $\begin{array}{l}7.9 \\
-\ldots \\
\ldots- \\
\ldots- \\
\ldots \\
\ldots\end{array}$ & $\begin{array}{l}7.6 \\
-\ldots \\
-- \\
-- \\
\ldots- \\
--\end{array}$ & $\begin{array}{l}7.8 \\
- \\
- \\
- \\
- \\
-\end{array}$ & $\begin{array}{l}7.6 \\
7.9 \\
8.2 \\
8.3 \\
8.2 \\
8.1\end{array}$ & $\begin{array}{l}7.2 \\
7.5 \\
7.7 \\
7.7 \\
7.3 \\
7.3\end{array}$ & $\begin{array}{l}7.5 \\
7.7 \\
8.0 \\
8.0 \\
7.8 \\
7.7\end{array}$ \\
\hline Month & $\ldots$ & $\cdots$ & $\cdots$ & $\cdots$ & $\cdots$ & $\cdots$ & $\cdots$ & $\cdots$ & $\cdots$ \\
\hline
\end{tabular}


Table 30. Daily maximum, minimum, and mean dissolved oxygen concentrations at station 403133080372801, from the New Cumberland Dam (downstream) continuous recording water-quality monitor, June to october 1995, Continued.

[-., value not determined]

\begin{tabular}{|c|c|c|c|c|c|c|c|c|c|}
\hline \multirow{3}{*}{ Day } & \multicolumn{9}{|c|}{ Dissolved oxygen $\mathrm{cc}$} \\
\hline & \multicolumn{3}{|c|}{ August } & \multicolumn{3}{|c|}{ September } & \multicolumn{3}{|c|}{ October } \\
\hline & Maximum & Minimum & Mean & Maximum & Minimum & Mean & Maximum & Minimum & Mean \\
\hline $\begin{array}{l}1 \\
2 \\
3 \\
4 \\
5\end{array}$ & $\begin{array}{l}7.9 \\
8.1 \\
7.8 \\
7.5 \\
7.1\end{array}$ & $\begin{array}{l}7.6 \\
7.3 \\
7.3 \\
7.1 \\
7.0\end{array}$ & $\begin{array}{l}7.8 \\
7.7 \\
7.5 \\
7.3 \\
7.1\end{array}$ & $\begin{array}{l}7.5 \\
7.8 \\
7.8 \\
8.3 \\
7.7\end{array}$ & $\begin{array}{l}7.0 \\
6.9 \\
6.9 \\
6.8 \\
7.1\end{array}$ & $\begin{array}{l}7.2 \\
7.3 \\
7.3 \\
7.3 \\
7.4\end{array}$ & $\begin{array}{l}8.9 \\
9.0 \\
\ldots- \\
\ldots- \\
\ldots-\end{array}$ & $\begin{array}{l}8.5 \\
8.6 \\
\ldots .- \\
\ldots- \\
\ldots-\end{array}$ & $\begin{array}{l}8.8 \\
8.8 \\
-\ldots \\
\ldots- \\
\ldots\end{array}$ \\
\hline $\begin{array}{r}6 \\
7 \\
8 \\
9 \\
10\end{array}$ & $\begin{array}{l}7.2 \\
7.4 \\
7.8 \\
8.1 \\
8.1\end{array}$ & $\begin{array}{l}6.9 \\
6.9 \\
7.2 \\
7.4 \\
7.4\end{array}$ & $\begin{array}{l}7.1 \\
7.1 \\
7.6 \\
7.8 \\
7.8\end{array}$ & $\begin{array}{l}8.0 \\
7.7 \\
7.4 \\
7.2 \\
7.7\end{array}$ & $\begin{array}{l}7.0 \\
6.6 \\
6.5 \\
6.6 \\
7.0\end{array}$ & $\begin{array}{l}7.3 \\
7.1 \\
7.1 \\
6.9 \\
7.4\end{array}$ & $\begin{array}{l}\cdots \\
\cdots \\
\cdots \\
\cdots \\
\cdots\end{array}$ & $\begin{array}{l}\cdots \\
\cdots \\
\cdots \\
\cdots \\
\cdots\end{array}$ & $\begin{array}{l}\cdots \\
\cdots \\
\cdots \\
- \\
-\end{array}$ \\
\hline $\begin{array}{l}11 \\
12 \\
13 \\
14 \\
15\end{array}$ & $\begin{array}{l}8.2 \\
8.3 \\
7.9 \\
8.1 \\
8.0\end{array}$ & $\begin{array}{l}7.6 \\
7.8 \\
7.7 \\
7.8 \\
7.7\end{array}$ & $\begin{array}{l}8.0 \\
8.1 \\
7.8 \\
7.9 \\
7.9\end{array}$ & $\begin{array}{l}7.8 \\
7.2 \\
7.6 \\
7.5 \\
7.6\end{array}$ & $\begin{array}{l}6.7 \\
6.8 \\
7.0 \\
6.9 \\
7.2\end{array}$ & $\begin{array}{l}7.3 \\
7.1 \\
7.3 \\
7.3 \\
7.4\end{array}$ & $\begin{array}{l}\cdots \\
\cdots \\
\cdots \\
\cdots \\
\cdots\end{array}$ & $\begin{array}{l}\cdots \\
\cdots \\
\cdots \\
\cdots \\
\cdots\end{array}$ & $\begin{array}{l}\cdots \\
\cdots \\
\cdots \\
\cdots \\
\cdots\end{array}$ \\
\hline $\begin{array}{l}16 \\
17 \\
18 \\
19 \\
20\end{array}$ & $\begin{array}{l}8.0 \\
8.2 \\
7.9 \\
7.8 \\
7.9\end{array}$ & $\begin{array}{l}7.7 \\
7.8 \\
7.5 \\
7.4 \\
7.3\end{array}$ & $\begin{array}{l}7.9 \\
7.9 \\
7.7 \\
7.5 \\
7.6\end{array}$ & $\begin{array}{l}7.4 \\
7.4 \\
7.3 \\
7.4 \\
7.4\end{array}$ & $\begin{array}{l}7.1 \\
7.0 \\
7.0 \\
6.9 \\
7.1\end{array}$ & $\begin{array}{l}7.3 \\
7.2 \\
7.2 \\
7.2 \\
7.2\end{array}$ & $\begin{array}{l}\ldots . \\
\cdots-. \\
9.3 \\
9.2 \\
9.4\end{array}$ & $\begin{array}{l}\cdots \\
\cdots \\
9.0 \\
9.0 \\
8.8\end{array}$ & $\begin{array}{l}\ldots . \\
\cdots \\
9.1 \\
9.1 \\
9.2\end{array}$ \\
\hline $\begin{array}{l}21 \\
22 \\
23 \\
24 \\
25\end{array}$ & $\begin{array}{l}7.8 \\
7.7 \\
8.1 \\
7.9 \\
8.3\end{array}$ & $\begin{array}{l}7.2 \\
7.2 \\
7.4 \\
7.3 \\
7.4\end{array}$ & $\begin{array}{l}7.5 \\
7.4 \\
7.7 \\
7.6 \\
7.7\end{array}$ & $\begin{array}{l}7.5 \\
7.6 \\
8.0 \\
8.1 \\
8.0\end{array}$ & $\begin{array}{l}7.1 \\
7.0 \\
7.5 \\
7.7 \\
7.6\end{array}$ & $\begin{array}{l}7.3 \\
7.3 \\
7.8 \\
7.9 \\
7.8\end{array}$ & $\begin{array}{l}9.5 \\
9.5 \\
9.8 \\
9.8 \\
9.9\end{array}$ & $\begin{array}{l}9.2 \\
9.4 \\
9.5 \\
9.6 \\
9.6\end{array}$ & $\begin{array}{l}9.4 \\
9.5 \\
9.6 \\
9.7 \\
9.8\end{array}$ \\
\hline $\begin{array}{l}26 \\
27 \\
28 \\
29 \\
30 \\
31\end{array}$ & $\begin{array}{l}8.4 \\
8.3 \\
8.6 \\
8.1 \\
7.8 \\
8.0\end{array}$ & $\begin{array}{l}7.3 \\
7.3 \\
7.4 \\
7.2 \\
7.2 \\
6.9\end{array}$ & $\begin{array}{l}7.6 \\
7.7 \\
7.8 \\
7.6 \\
7.5 \\
7.4\end{array}$ & $\begin{array}{l}8.2 \\
8.3 \\
8.6 \\
8.8 \\
8.9 \\
-.-\end{array}$ & $\begin{array}{l}7.7 \\
7.7 \\
8.1 \\
8.4 \\
8.4 \\
-.-\end{array}$ & $\begin{array}{l}8.0 \\
8.0 \\
8.4 \\
8.7 \\
8.7 \\
-. .\end{array}$ & $\begin{array}{l}10.0 \\
10.1 \\
10.0 \\
10.2 \\
10.3 \\
10.3\end{array}$ & $\begin{array}{r}9.7 \\
9.9 \\
9.9 \\
9.9 \\
10.1 \\
10.1\end{array}$ & $\begin{array}{r}9.9 \\
10.0 \\
9.9 \\
10.1 \\
10.2 \\
10.2\end{array}$ \\
\hline Month & 8.6 & 6.9 & 7.6 & 8.9 & 6.5 & 7.5 & $\ldots$ & $\ldots$ & $\ldots$ \\
\hline
\end{tabular}




\section{CONVERSION FACTORS AND ABBREVIATIONS}

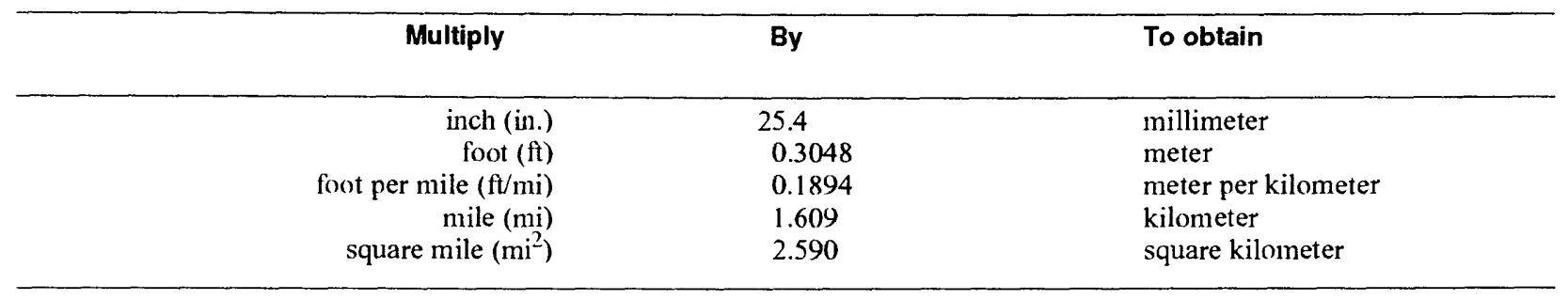

Temperature is given in degrees Celsius $\left({ }^{\circ} \mathrm{C}\right)$, which can be converted to degrees Fahrenheit $\left({ }^{\circ} \mathrm{F}\right)$ by use of the following equation:

$$
\mathrm{F}=1.8\left({ }^{\circ} \mathrm{C}\right)+32
$$

River Mile: A unit of length applied to the main stem of a river to denote location. Typically, the mouth of a river is designated river mile zero and river mile length is measured upstream from this point. River mile zero on the Ohio River has been designated as the river's origin in Pittsburgh, Pa., however, and river mile length is measured downstream from this point.

Abbreviated water-quality units used in this report: Chemical concentrations and water temperature are given in metric units. Chemical concentration is given in grams per liter $(\mathrm{g} / \mathrm{L})$, milligrams per liter $(\mathrm{mg} / \mathrm{L})$, or micrograms per liter $(\mu \mathrm{g} / \mathrm{L})$. Milligrams per liter is a unit expressing the concentration of chemical constituents in solution as weight (milligrams) of solute per unit volume (liter) of water. One thousand milligrams per liter is equivalent to one gram per liter. One thousand micrograms per liter is equivalent to one milligram per liter. For concentrations less than $7,000 \mathrm{mg} / \mathrm{L}$, the numerical value is the same as for concentrations in parts per million.

Specific conductance of water is expressed in microsiemens per centimeter at 25 degrees Celsius $(\mu \mathrm{S} / \mathrm{cm})$. This unit is equivalent to micromhos per centimeter at 25 degrees Celsius $(\mu \mathrm{mho} / \mathrm{cm})$, formerly used by the U.S. Geological Survey. 